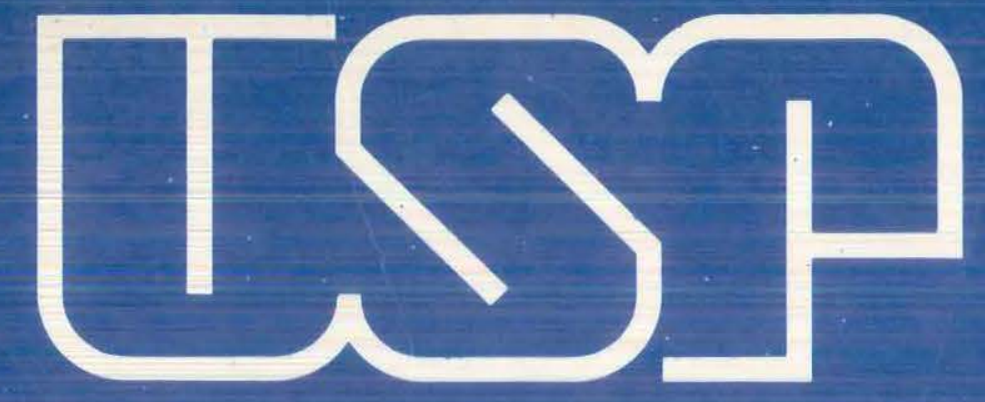

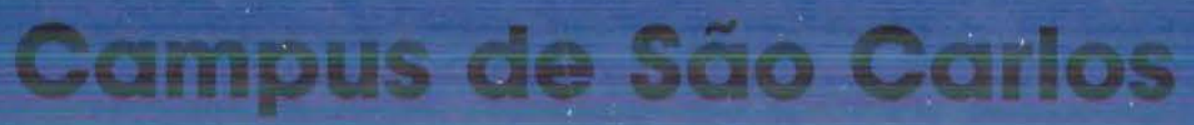

ANÁLISE DE PLACAS COM VARIAÇÃO DE ESPESSURA ATRAVÉS DO MÉTODO DOS ELEMENTOS DE CONTORNO

AUTOR: ENG. EDUARDO WALTER VIEIRA CHAVES ORIENTADOR: PROF. DR. WILSON SÉRGIO VENTURINI

UNIVERSIDADE DE SÃO PAULO 


\section{ANÁLISE DE PLACAS COM \\ VARIAÇÃO DE ESPESSURA ATRAVÉS DO \\ MÉTODO DOS ELEMENTOS DE CONTORNO}

\section{EDUARDO WALTER VIEIRA CHAVES}

Dissertação apresentada à Escola de Engenharia de São Carlos, da Universidade de São Paulo, como parte dos requisitos para obtenção do Título de Mestre em Engenharia de Estruturas.

ORIENTADOR: Prof. Tit. Wilson Sergio Venturini

\section{São Carlos}

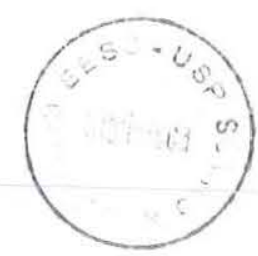


Clase. TE SC- ECSC

cutt. 1494

Tombo $1 6 6 \longdiv { 9 7 }$

st 0927491

Ficha catalográfica preparada pela Seção de Tratamento da Informação do Serviço de Biblioteca - EESC-USP

Chaves, Eduardo Walter Vieira

C512a Análise de placas com variação de espessura através do método dos elementos de contorno /

Eduardo Walter Vieira Chaves. -- São Carlos, 1997.

Dissertação (Mestrado). - Escola de Engenharia

de São Carlos-Universidade de São Paulo, 1997.

Área: Engenharia de Estruturas.

Orientador: Prof. Tit. Wilson Sérgio Venturini.

1. Placas. 2. Elementos de contorno. 3. Rigidez variável. I. Título 
"..., nothing that has once been discovered ever loses its value or has to be discardedi; ..."

LOVE, A.E. 
Aos meus avós paternos e

maternos e aos meus pais:

Luciano Walter e Maria José,

pois a vida me concederam. 


\section{AGRADECIMENTOS}

Ao Prof. Wilson Sergio Venturini, pela extraordinária orientação e paciência dedicadas a este trabalho, sem as quais seria dificil sua finalização. Agradeço-lhe também por me ensinar que as coisas dificeis podem se tornar fáceis.

Aos professores do Departamento de Engenharia de Estruturas da Escola de Engenharia de São Carlos/USP, em especial ao Prof. Dr. João Batista de Paiva.

Aos professores do Departamento de Engenharia Civil da UFPE, em especial ao Prof. Dr. Sílvio Romero Frej da Fonseca Lima, pela orientação durante a graduação e pela amizade.

Aos meus eternos professores: meus pais.

A Fernando, Anna, Mauro, Andréa, Gustavo, Débora e Carol, pelo sangue que nos une e por me apoiarem quando precisei, apesar da distância que nos separa.

Ao amigo e colega Eng. Rodrigo de Carvalho Soares pelo companheirismo e amizade durante a graduação e o mestrado.

Aos colegas do departamento em especial a: Eng ${ }^{\text {a. }}$ Ana Maria Brandão pela amizade e pelo apoio técnico dispensado nesta dissertação; Eng. Alex de Sousa e Eng. Osvaldo de Holanda pela hospitalidade.

Aos funcionários do Departamento de Engenharia de Estruturas da Escola de Engenharia de São Carlos, especialmente a Rosi Aparecida Jordão Rodrigues, Maria Nadir Minatel e Rui Roberto Casale.

Aos funcionários da Biblioteca Central, em especial a Milna Aiello, por fazer mais pelos alunos.

Ao $\mathrm{CNPq}$ e à FAPESP que possibilitaram, através de bolsas de estudos, o desenvolvimento desta dissertação. 


\section{SUMÁRIO}

LISTA DE FIGURAS ................................................................................... viii

LISTA DE TABELAS ................................................................................

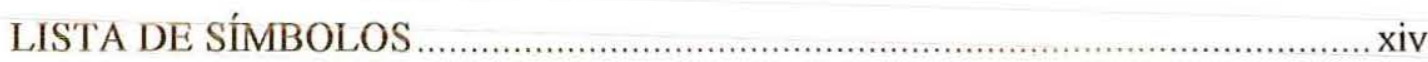

LISTA DE ABREVIATURAS E SIGLAS .......................................................... xviii

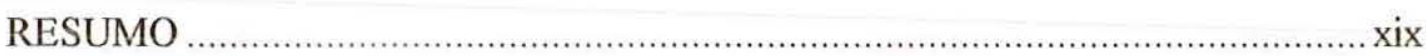

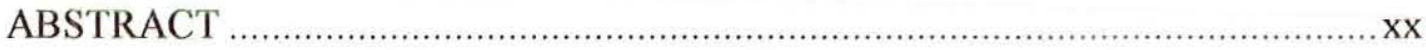

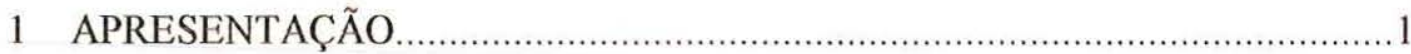

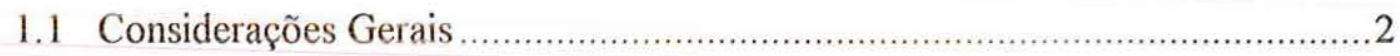

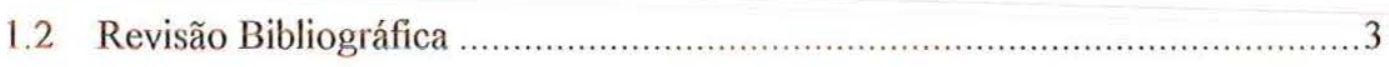

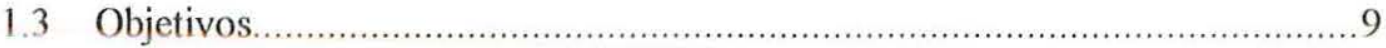

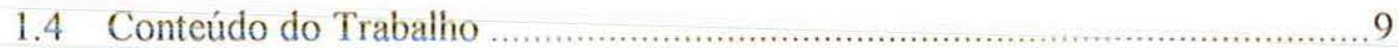

2 RELAÇÕES BÁSICAS: TEORIA DA ELASTICIDADE E PLACAS .............. 10

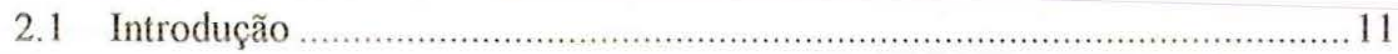

2.2 Relação Deformação-Deslocamento .......................................................... 11

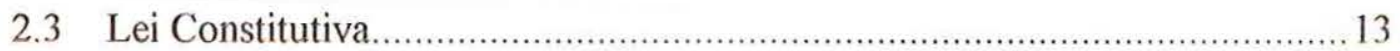

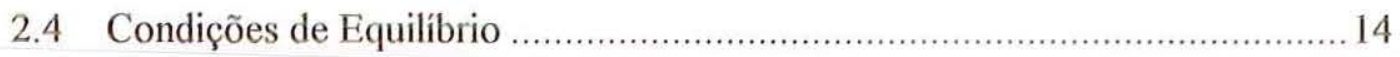

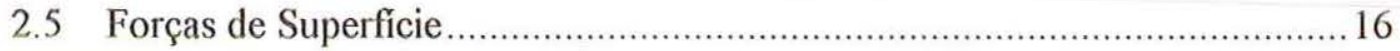

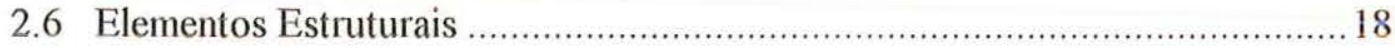

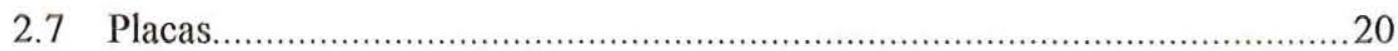

2.8 Hipóteses e Relações Básicas da Teoria de Kirchhoff ..................................23

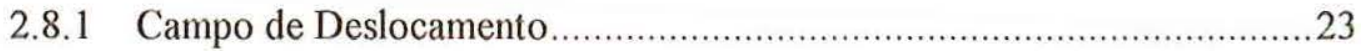

2.8.2 Campo de Deformação................................................................................25 


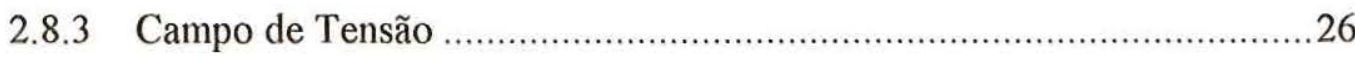

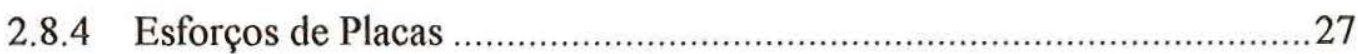

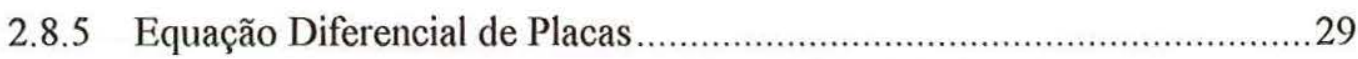

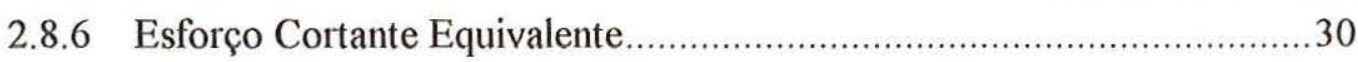

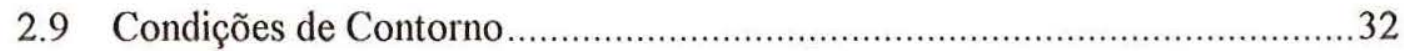

2.10 Placa com Espessura Variável ........................................................................33

2.11 Esforços Segundo um Sistema Genérico de Coordenadas ........................... 35

2.12 Equações de Placas em Coordenadas Polares .......................................... 38

2.13 Soluções Fundamentais de Placas ...................................................................45

3 EQUAÇÕES INTEGRAIS PARA FLEXÃO DE PLACA …................................55

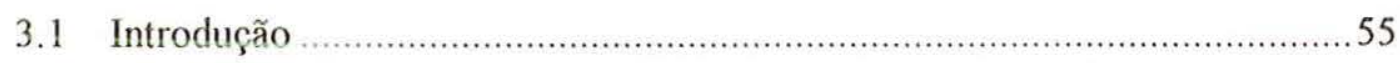

3.2 Equação Integral de um Ponto do Domínio da Placa com Espessura

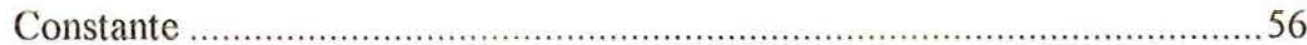

3.3 Equação Integral de um Ponto do Domínio da Placa de Espessura

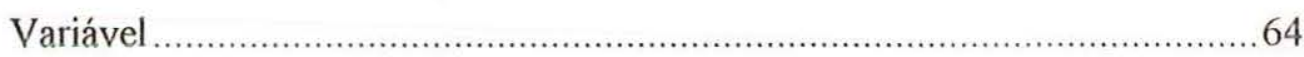

3.4 Equação Integral para um Ponto do Contorno da Placa ............................... 70

4 EQUAÇÕES ALGÉBRICAS DO MEC PARA PLACA DE KIRCHHOFF ........80

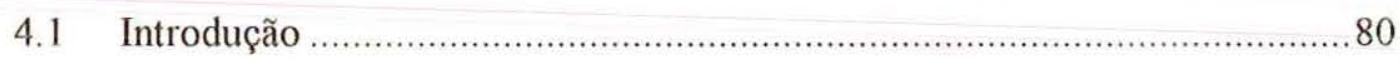

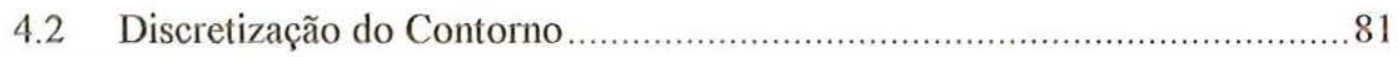

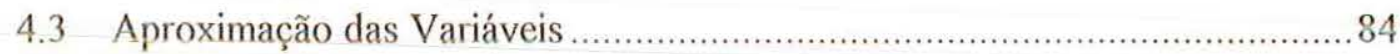

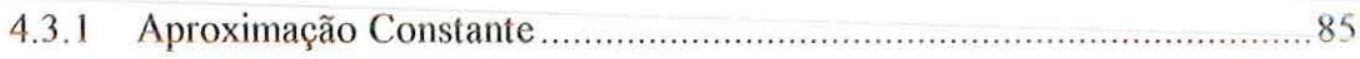

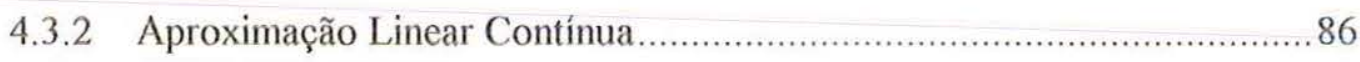

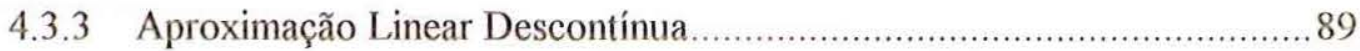

4.3.4 Aproximação Linear Mista ................................................................ 90

4.3.5 Aproximação Quadrática Contínua .................................................... 91

4.3.6 Aproximação Quadrática Descontínua................................................99

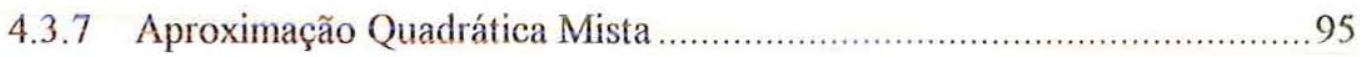

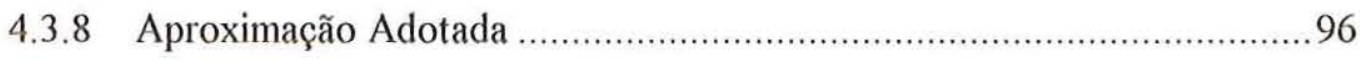

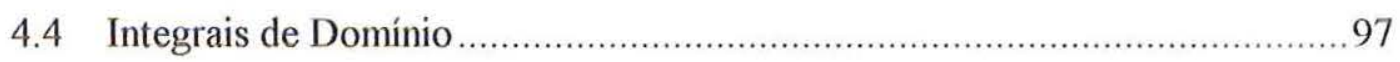

4.4.1 Integrais de Domínio para o Carregamento.............................................. 99

4.4.2 Integrais de Domínio sobre as Células ............................................... 103 
4.5 Definição das Posições do Ponto de Colocação .......................................... 109

4.6 Transformação das Equações Integrais em Equações Algébricas ............... 113

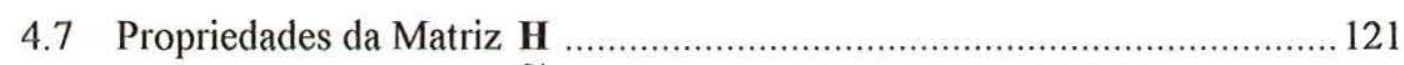

4.8 Deslocamentos e Esforços para Pontos Internos ........................................ 123

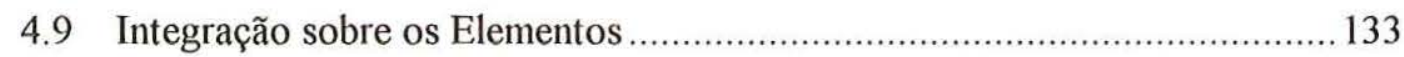

4.9.1 Integração Numérica ........................................................................ 133

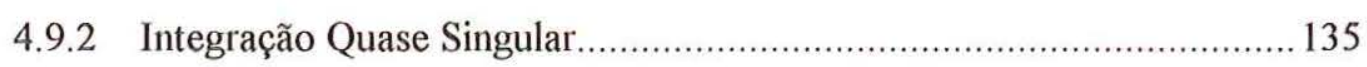

4.9.3 Integração Singular .......................................................................... 135

4.10 Representações Algébricas Alternativas.................................................. 148

5 RESULTADOS E CONCLUSÃO .............................................................. 150

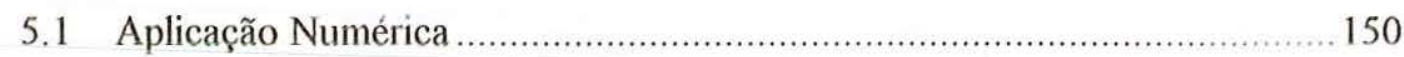



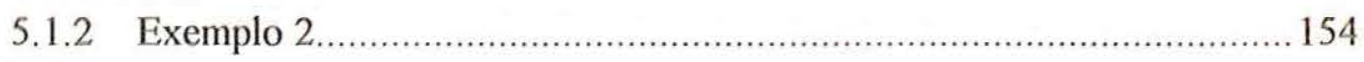

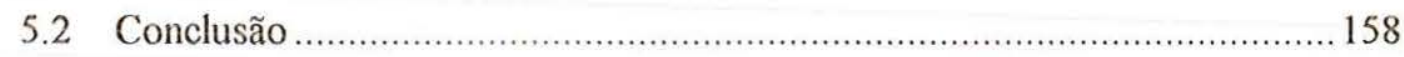

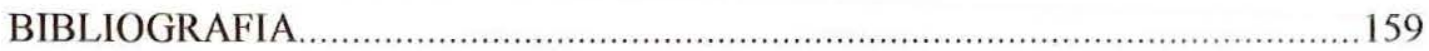




\section{LISTA DE FIGURAS}

FIGURA 2.1 - Deformação de um Sólido Tridimensional

FIGURA 2.2 - Campo de Tensões em Três Dimensões.

FIGURA 2.3 - Tensões e Forças de Superfície num Tetraedro Infinitesimal.

FIGURA 2.4 - Co-senos Diretores do Vetor ON.

FIGURA 2.5 - Tensões e Forças de Superfície no Caso Bidimensional.

FIGURA 2.6 - Exemplo de Placa

FIGURA 2.7 - Exemplo de Chapa.

FIGURA 2.8 - Exemplo de Casca.

FIGURA 2.9 - Exemplo de Sapata.

FIGURA 2.10 - Placa Delgada de Espessura t.

FIGURA 2.11 - Plano Médio Indeformado da Placa.

FIGURA 2.12 - Plano Médio Deformado Conforme Teoria de Kirchhoff.

FIGURA 2.13 - Plano Médio Deformado Conforme Teoria de Reissner-Mindlin. ...22

FIGURA 2.14 - Deslocamento Positivo de um Ponto Qualquer. 
FIGURA 2.15 - Placa Antes e Após o Carregamento.

FIGURA 2.16 - Distribuição de Tensões. 27

FIGURA 2.17 - Esforços Atuantes em um Elemento Infinitesimal de Placa. .28

FIGURA 2.18 - Esforços em um Elemento Diferencial $\left(\mathbf{d x}_{\mathbf{1}} \cdot \mathbf{d} \mathbf{d x}_{\mathbf{2}}\right)$ de Placa.

FIGURA 2.19 - Condições nas bordas.

FIGURA 2.20 - Reação de Canto.

FIGURA 2.21 - Placa com Condições de Contorno. 33

FIGURA 2.22 - Variação Linear da Rigidez. 34

FIGURA 2.23 - Sistemas de Coordenadas $\left(\mathbf{x}_{1}, \mathbf{x}_{2}\right)$ e $(\mathbf{n}, \mathbf{s})$ 35

FIGURA 2.24 - Tensões e Esforços no Elemento "abc". 36

FIGURA 2.25 - Momento Volvente na Borda. 38

FIGURA 2.26 - Sistema de Coordenadas Cartesianas e Polares. 38

FIGURA 2.27 - Vetores n e s no Ponto P do Contorno da Placa. 42

FIGURA 2.28 - Resposta em "p" Devido a Distribuição Delta de Dirac $\delta(q, p) \ldots . .45$

FIGURA 2.29 - Função Delta de Dirac. 46

FIGURA 2.30 - Forças Atuantes no Círculo de Raio r e Centro q. 48

FIGURA 2.31 - Pontos de Carregamento "q" e de Resposta " $p$ ". 50

FIGURA 2.32 - Sistema de Coordenadas $(\mathrm{n}, \mathrm{s})$ e $(\mathrm{m}, \mathrm{u})$. 52

FIGURA 3.1 - Placa Finita Contida em uma Placa Infinita, $\Omega \in \Omega_{\infty}$ 56

FIGURA 3.2 - Estados de Carregamentos Virtual g* e Real g. 57 
FIGURA 3.3 - Sistema de Coordenadas (n,s), Normal e Tangente ao Contorno. .....58

FIGURA 3.4 - Momentos Volventes em um Canto i da Placa. 60

FIGURA 3.5 - Contorno Circular Acrescentado a um Ponto Q. 70

FIGURA 4.1 - Discretização do Contorno da Placa. 81

FIGURA 4.2 - Geometria dos Elementos de Contorno. 82

FIGURA 4.3 - Elemento de Contorno Linear. 83

FIGURA 4.4 - Aproximação Constantes. 86

FIGURA 4.5 - Aproximação Linear Contínua 86

FIGURA 4.6 - Funções de Forma para Aproximação Linear Contínua das Variáveis. 88

FIGURA 4.7 - Descontinuidade do Contorno de uma Placa. 89

FIGURA 4.8 - Função de Forma Linear Descontínua para as Variáveis. .90

FIGURA 4.9 - Função de Forma Linear Mista para as Variáveis. 91

FIGURA 4.10 - Aproximação Quadiática Contínua das Variáveis no Contorno......92

FIGURA 4.11 - Funções de Forma para Aproximação Quadrática Contínua das Variáveis

FIGURA 4.12 - Funções Interpoladoras Quadráticas Descontínuas. .95

FIGURA 4.13 - Nós Duplos. 96

FIGURA 4.14 - Ponto de Colocação. 97

FIGURA 4.15 - Placa com Carregamento Distribuído. 98

FIGURA 4.16 - Divisão do Domínio em Células Triangulares. .99 


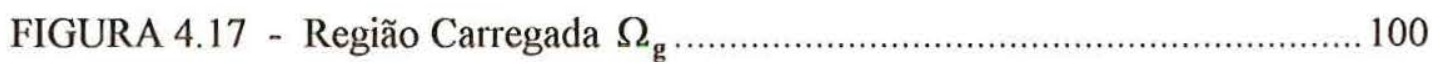

FIGURA 4.18 - Discretização do Contorno do Carregamento. .............................. 103

FIGURA 4.19 - Definição do Sistema de Coordenadas de uma Células Triangular “i”.104

FIGURA 4.20 - Célula Interna e os Sistemas de Coordenadas $\left(\mathbf{x}_{1}, \mathbf{x}_{2}\right),\left(\xi_{1}, \xi_{2}\right)$ e $(\mathbf{r}, \theta)$. 106

FIGURA 4.21 - Posições do Ponto de Colocação.

FIGURA 4.22 - Coeficiente de Posicionamento a.

FIGURA 4.23 - Recuo do Nó Duplo.

FIGURA 4.24 - Função Quadrática no Elemento de Integração

FIGURA 4.25 - Movimento de Corpo Rígido - Translação.

FIGURA 4.26 - Movimento de Corpo Rígido - Rotação.

FIGURA 4.27 - Funções de Forma Global $\phi_{\mathrm{n}}$

FIGURA 4.28 - Coordenadas Adimensionais $\eta$ e $\xi$.

FIGURA 4.29 - Elemento de Contorno Linear Descontínuo.

FIGURA 4.30 - Variação Linear da Rigidez no Contorno da Placa.

FIGURA 4.31 - Posição do Ponto Singular $\xi_{\mathrm{s}}$

FIGURA 4.32 - Função de Forma $\phi_{1}$.

FIGURA 4.33 - Função de Forma $\phi_{2}$.

FIGURA 4.34 - Função de Forma $\phi_{3}$. 
FIGURA 5.1 - Placa Quadrada, de Espessura Constante, Apoiada em Dois Lados Opostos e Engastada nos Outros Dois.

FIGURA 5.2 - Placa Quadrada Apoiada em Dois Lados Opostos e Livre nos Outros Dois. 


\section{LISTA DE TABELAS}

TABELA 5.1 - Discretização Via Elemento de Contorno -

Placa de Espessura Constante. 152

TABELA 5.2 - Discretização Via Elemento Finito. 152

TABELA 5.3 - Discretização Via Elemento de Contorno -

Placa de Espessura Constante. .155 


\section{LISTA DE SÍMBOLOS}

$\nabla^{2} \quad:$ operador diferencial escalar, $\nabla^{2}=\frac{\partial^{2}}{\partial \mathbf{x}_{1}^{2}}+\frac{\partial^{2}}{\partial \mathbf{x}_{2}^{2}}+\frac{\partial^{2}}{\partial \mathbf{x}_{3}^{2}}$

$\Gamma \quad$ : coordenada que percorre o contorno;

$\Gamma_{1}, \Gamma_{2} \quad$ : coordenadas dos limites do contorno no qual se realiza a integração;

$\Gamma_{\infty} \quad$ : contorno infinito;

$\Gamma_{\mathbf{j}} \quad$ : elemento de contorno;

$\Omega \quad$ : coordenada de domínio;

$\Omega_{\mathrm{g}} \quad$ : área do carregamento distribuído;

$\Omega_{\varepsilon} \quad$ : região do domínio;

$\beta_{\mathbf{c}} \quad$ : angulosidade do canto da placa;

$\Omega_{\infty} \quad$ : domínio infinito;

$\delta_{\mathrm{ij}} \quad$ delita de Kronecker;

$\delta(q, p) \quad:$ delta de Dirac;

$\lambda, \mu \quad$ : constantes de Lamé;

$v \quad:$ coeficiente de Poisson;

$\xi \quad$ : ponto onde se aplica a equação integral;

$\phi^{\mathrm{T}} \quad$ : matriz que contém as funções de forma;

$\sigma_{\mathrm{ij}} \quad:$ tensor das tensões;

$\mathbf{b}_{\mathbf{i}} \quad$ : forças volumétricas;

$\mathbf{C}_{\mathrm{ijk} \ell} \quad:$ tensor de quarta ordem que contém as constantes elásticas; 
$\mathbf{D}_{\mathbf{c i}} \quad$ : rigidez à flexão da placa correspondente ao canto;

Do : rigidez à flexão da placa fundamental;

$\mathbf{D}\left(\mathbf{x}_{1}, \mathbf{x}_{2}\right)$ : variação da rigidez à flexão da placa;

E : módulo de elasticidade longitudinal do material;

g : carregamento uniformemente distribuído;

$\mathrm{g}^{*} \quad$ : carregamento fundamental, geralmente é o delta de Dirac;

$\mathbf{t}\left(\mathbf{x}_{1}, \mathbf{x}_{2}\right)$ : espessura variável da placa;

$\mathbf{m}_{\mathbf{i j}} \quad:$ tensor dos momentos internos;

$\mathbf{M}_{\mathbf{i}} \quad$ : Vetor que contém os momentos no contorno;

$\overline{\mathbf{M}}_{\mathbf{n}} \quad$ : momento externo por unidade de comprimento para a flexão na direção normal ao contorno;

$\mathbf{M}_{\mathrm{s}} \quad$ : momento externo por unidade de comprimento para a flexão na direção tangencial ao contorno;

$\mathbf{M}_{\text {ns }} \quad$ : momento volvente externo por unidade de comprimento;

n : vetor unitário normal ao contorno no plano da placa;

$n_{i} \quad:$ co-senos diretores da normal em relação ao eixo i;

$\mathrm{N}_{\mathrm{c}} \quad$ : número total de cantos no contorno da placa;

$\mathbb{N}_{\text {cal }} \quad$ : número total de células

$\mathrm{N}_{\mathrm{el}} \quad$ : número de elementos de contorno;

$\mathrm{N}_{\mathrm{n}} \quad$ : número total de nós de contorno;

Npi : número total de pontos internos;

$\mathrm{N}_{\text {subel }} \quad$ : número de subelementos

$\mathrm{p}_{\mathrm{i}} \quad$ : componentes das forças de superficies;

p : vetor das forças internas ao elemento;

P : vetor das forças em todos os nós;

$\mathbf{q}_{\mathbf{i}} \quad$ : são as forças cortantes segundo as direções do sistema global de coordenadas; 
r : distância de onde se aplicou o carregamento unitário ao ponto onde se deseja obter a força ou deslocamento na solução fundamental;

$\mathbf{r}, \theta \quad$ : sistema de coordenadas polares;

R : raio de curvatura do contorno no ponto $\mathrm{P}$;

$\mathbf{R}_{\mathbf{c}} \quad$ : reação de canto;

$\mathbf{R}_{\mathbf{c}}^{*} \quad$ : valor de $\mathbf{R}_{\mathbf{c}}$ na solução fundamental;

s

: vetor unitário tangente ao contorno no plano da placa;

T : matriz de transformação de coordenadas;

$\mathbf{u}_{1}, \mathbf{u}_{2}, \mathbf{u}_{3} \quad$ : componentes de deslocamentos correspondente ao sistema cartesiano $\mathrm{x}, \mathrm{y}, \mathrm{z}$;

$\stackrel{\mathbf{u}}{\sim}$

: vetor dos deslocamentos internos ao elemento;

$\underset{\sim}{U} \quad$ : vetor dos deslocamentos em todos os nós;

$\varepsilon_{i j} \quad:$ representa as componentes de deformação no sistema global;

$\mathbf{V}_{\mathbf{n}} \quad$ : cortante equivalente por unidade de comprimento;

$\mathbf{V}_{n}^{*} \quad$ : valor de $\mathbf{V}_{n}$ na solução fundamental;

w: deslocamento na direção do eixo $\mathrm{x}_{3}$;

$w_{\mathrm{c}} \quad:$ desiocamento do canto da placa;

$w^{*} \quad$ : valor de $w$ na solução fundamental;

$w_{\mathrm{c}}^{*} \quad$ : deslocamento fundamental do canto i da placa;

$\partial w / \partial \mathbf{n} \quad$ : rotação normal ao contorno

$\partial w / \partial \mathrm{s} \quad$ : rotação tangencial ao contorno;

$\partial w / \partial \mathrm{x}_{1} \quad$ : rotação contida na direção do eixo $\mathrm{x}_{1}$;

$\partial w / \partial \mathrm{x}_{2} \quad$ : rotação contida na direção do eixo $\mathbf{x}_{2}$;

$\partial w^{*} / \partial \mathbf{n} \quad:$ valor de $\partial w / \partial \mathbf{n}$ na solução fundamental;

$w_{9, \quad \text { : curvaturas; }}$

$w_{,_{\mathrm{ij}}}^{*} \quad$ : valor de $w_{\mathbf{i}_{\mathrm{j}}}$ na solução fundamental; 
$\mathbf{x}_{1}, \mathbf{x}_{2}, \mathbf{x}_{3} \quad$ : sistema de coordenadas cartesianas;

x : vetor das coordenadas do nó do elemento;

$\underset{\sim}{\mathrm{X}^{N}} \quad$ : vetor de coordenadas do nó do elemento; 


\section{LISTA DE ABREVIATURAS E SIGLAS}

MDF - Método das Diferenças Finitas;

MEF - Método dos Elementos Finitos;

MEC - Método dos Elementos de Contorno;

DKT - Discrete Kirchhơf Theory 


\section{RESUMO}

CHAVES, E.W.V. (1997). Análise de placas com variação de espessura através do método dos elementos de contorno. São Carlos. 171p. Dissertação (Mestrado) - Escola de Engenharia de São Carlos, Universidade de São Paulo.

Este trabalho trata da formulação do Método dos Elementos de Contorno para o problema de flexão de placas através da teoria clássica de Kirchhoff. Ênfase especial é dada a placas com variação de espessura uma vez que este tema é muito pouco abordado. O estudo de flexão de placas com variação de espessura é exercica em vários campos, tais como engenharia civil, engenharia aeroespacial e projeto de máquinas. Adotou-se uma variação linear da rigidez, resultando nas equações integrais de deslocamentos com termos de domínios, que serão tratados por discretização do domínio em células.

Palavras-chave: Placas - elementos de contorno - rigidez variável. 


\section{ABSTRACT}

CHAVES, E.W.V. (1997). Análise de placas, considerando variação de espessura, através do método dos elementos de contorno. São Carlos, 1997. 271p. Dissertação (Mestrado) - Escola de Engenharia de São Carlos, Universidade de São Paulo.

This work deals with the formulation of the Boundary Element Method applied to plate bending problem using the Kirchhoff's theories. Special emphasis is given to plates with varying thickness since this subject is not much tackled. The study of the bending of plates of variable thickness is pursued in various fields, such as civil engineering, aerospace engineering, and the design of machines. It was adopted linearly varying rigidity, giving integral equations of displacements with domain terms, that will be treated by domain discretization into cells.

Keywords: Plate bending - boundary elements - varying thickness. 


\section{APRESENTAÇÃO}

\subsection{Considerações Gerais}

A grande maioria dos problemas em engenharia é governada por equações diferenciais, as quais satisfazem as condições de equilíbrio e de compatibilidade, juntamente com condições de contorno apropriadas c condições iniciais. Podendo apresentar complexidade na geometria do sólido bem como materiais com leis constitutiva complexas. Como resultado soluções analíticas (exatas) desses problemas são praticamente impossíveis de serem obtidas; para contornar esses problemas têm sido utilizados métodos numéricos para a determinação de soluções aproximadas, que hoje são viáveis em muitos problemas complexos devido ao advento dos computadores. Um método numérico pode ser do tipo diferencial ou integral dependendo do tipo de representação feita para desenvolver o problema.

Os métodos numéricos mais largamente conhecidos e utilizados são o Método das Diferenças Finitas (MDF) e o Método dos Elementos Finitos (MEF), ambos do tipo diferencial, isto é: solução obtida a partir da equação diferencial do problema. O método das diferenças finitas é o mais antigo desses métodos, tendo surgido provavelmente no século passado. Sua sistematização pode ser vista em SOUTHWELL (1946), e é utilizado ainda hoje, na solução de diversos problemas de engenharia. Este método transforma equações diferenciais em equações algébricas válidas apenas nos nós dentro do domínio, através de aproximações das derivadas por diferenças finitas, enquanto que o MEF transforma o próprio domínio em uma série de subdomínios finitos ou elementos conectados através de seus nós cujas variáveis sobre cada elemento são governadas por funções aproximadoras contínuas. O MEF tem sua divulgação a partir dos trabalhos de TURNER et alii , em 1956, ARGYRIS (1969) e CLOUGH (1960). Apesar de ter sido COURANT (1943) quem primeiro 
usou o conceito de discretização da região a ser tratada para problemas de torção de St. Venant. A história mais detalhada sobre o MEF pode ser encontrada em CLOUGH (1990), ROBINSON (1985) e GUPTA \& MEEK (1996). O crescimento do método só foi possível devido ao avanço tecnológico dos equipamentos computacionais. Este método tornou-se o mais utilizado por diversas áreas da engenharia, mostrando-se extremamente eficiente para a solução de problemas práticos. Atualmente, é empregado em sistemas computacionais para a análise de estruturas, inclusive com a consideração de resposta não-lineares, que só foi possível devido a um melhor conhecimento do comportamento dos materiais. O MEF apresenta muitas vantagens sobre o MDF tais como melhor conformidade para a geometria do domínio, mais fácil aplicabilidade das condições de contorno e mais fácil construção de malhas de tamanho variável. Essas vantagens têm feito do MEF o método numérico mais utilizado entre cientistas e engenheiros, mesmo apresentando entrada e saída de dados trabalhosas, especialmente em problemas que apresentam o contorno bem complexo. Este tipo de problema pode ser contornado através de Pré e Pós- Processadores com geração automática de malhas com recursos gráficos.

O Método dos Elementos de Contorno (MEC), que é baseado na formulação integral do problema, tem emergido há 30 anos como uma ferramenta computacional poderosa. Este método necessita apenas da discretização do contorno e não da discretização do domínio como no MDF e MEF. Esse fato faz o MEC mais eficiente do que o MEF para um grande número de problemas, embora em certos casos o MEC necessita-se também da discretização do domínio em células para sua completa formulação. Para alguns problemas a combinação dos dois métodos cria um arranjo numérico excelente. O MEC tem sido utilizado com grande sucesso em problemas de elastostática, potencial, problemas elastoplásticos , viscoplasticidade, elastodinâmica, análise da fratura, condução de calor, electromagnetismo , interação solo-estrutura , interação estrutura-fluido e etc. O MEC só pode ser aplicado se uma solução fundamental da equação diferencial é conhecida, as conhecidas funções de Green. Basicamente existem dois tipos de MEC: Direto e Indireto.

- Direto: Nesta formulação as variáveis físicas reais do problema aparecem nas equações integrais. 
- Indireto: $\mathrm{Na}$ formulação indireta as equações integrais são expressas completamente em termo de uma solução singular unitária da equação diferencial original. Para isso utiliza-se variáveis fictícias distribuídas no contorno que não tem significado físico.

\subsection{Revisão Bibliográfica}

Apesar de apenas nas últimas três décadas ter crescido o interesse dos pesquisadores pelo MEC, as equações integrais, base do desenvolvimento dessa técnica, são conhecidas há muito tempo. Segundo ELLIOT, foi ABEL, em 1823, quem primeiro deduziu uma equação integral para o tratamento de um problema físico, o pêndulo isôcrono. Posteriormente, em 1837, LIOUVILLE transformou um problema de valor inicial em uma equação integral, que a resolveu por aproximações sucessivas. Foi em 1903 que FREDHOLM publicou um trabalho rigoroso sobre equações integrais. Até 1950, apenas problemas de valores de contorno relativos a casos particulares foram estudados, baseados sempre nas equações integrais lineares de Fredholm. A introdução do segundo problema fundamental de contorno para o campo das análises elásticas é devida a KUPRADZE (1965), que utilizou a teoria de Fredholm em equações com integrais singulares. Os trabalhos notáveis de autores russos como MUSKHELISHVILI (1953), MIKHLIN (1957), SMIRINOV (1964), GAKHOV (1966) e IVANOV (1976) deram enorme contribuição ao início de uma nova era do uso das equações integrais para resolução de problemas físicos.

Os métodos desenvolvidos por Trefftz e Prager em 1917 e 1928, respectivamente, para resolução de equações integrais na mecânica dos fluidos devem ser considerados como os precursores da moderna técnica de integração de contorno. O método da equação integral como uma ferramenta prática, geral e eficiente começa a aparecer na década de 60 , um período caracterizado pela difusão do uso dos computadores, observando-se uma considerável expansão e desenvolvimento do método durante a década de 70 . 
O trabalho de RIZZO (1967) é o primeiro que trata as equações integrais como forma de técnica numérica. Certamente esse trabalho é também o primeiro a propor a formulação direta para o tratamento das equações integrais do problema elástico. As formulações até então apresentadas são baseados no procedimento indireto. Inicialmente o método apareceu com o termo "BIEM (Boundary Integral Equation Method)" introduzido por RIZZO (1967) já como técnica alternativa das equações integrais. Outros autores seguindo a mesma terminologia como CRUSE (1969), (1973) e (1974). CRUSE \& RIZZO (1968) apresenta uma formulação do método das equações integrais de contorno, para análise de problema elastodinâmico usando transformadas de Laplace com relação ao tempo.

RIZZO \& SHIPPY (1968) utilizam o método das equações integrais de contorno, considerando a elasticidade linear e sugerindo o uso de sub-regiões para o tratamento dos domínios não homogêneos. Em 1971, CRUSE \& VANBUREN aplicaram pela primeira vez a formulação a um sólido tridimensional, considerando a influência de um "crack" e SWEDLOW \& CRUSE (1971) apresentaram uma formulação para simulação de materiais elastoplásticos, anisotrópicos e compressíveis, considerando ainda a relação tensão-deformação com encruamento. Partindo de Sweldlon \& Cruse, e utilizando outros critérios que permitiram a modelação dos efeitos integrais, RICCARDELLA (1973) aplica o método das equações integrais de contorno na análise tridimensional de tensões, apresentando, ainda, alguns exemplos numéricos. Este trabalho é considerado um marco dentro do método por ser o primeiro a resolver problemas no campo da elastoplasticidade, utilizando a formulação direta e por servir de base para trabalhos que o sucederam.

O emprego das equações integrais para a resolução de problemas potenciais, também de importância para a ciência da engenharia, é apresentado em trabalhos anteriores ao artigo de RIZZO (1967). JASWON (1963) e SYMM (1963) apresentaram um processo numérico para a resolução da equação integral de contorno de Fredholm.

O grande avanço nos chamados métodos de contorno tem sua origem na tese de LACHAT (1975), apresentada à Universidade de Southamptom, tratando de problemas bi e tridimensionais. Esse trabalho apresenta um eficiente tratamento 
numérico utilizando elementos curvos de segunda ordem com opções de variação linear, quadrática e cúbica para as aproximações das variáveis do problema. As integrais são calculadas numericamente por um sofisticado esquema de integração através das fórmulas da quadratura gaussiana. Foi em 1978 que BREBBIA usou a terminologia BEM (Boundary Element Method) que pode ser obtida como um caso especial de técnica dos resíduos ponderados, assim estabelecendo uma coneç̧ão entre as várias técnicas numéricas existentes.

A partir de 1978, com a publicação do primeiro livro de BREBBIA (1978), ainda que bastante simples, tornou-se o MEC mais conhecido e estudado em diversos centros importantes de pesquisa.

A primeira aplicação para problemas de flexão em placas, utilizando as equações integrais, foi introduzida por JASOW et alii (1967), que propôs a solução da equação biarmônica, via equação integral e posteriormente aplicou-se na solução de placas JASOW (1968).

ALTIERO \& SIKARSKIE (1978) apresentaram uma técnica que consiste em considerar a placa real contida em uma placa fictícia, cuja função de Green é conhecida. Posteriormente, WU \& ALTIERO (1979) estenderam esta técnica para incluir condições arbitrárias de contorno. Um trabalho similar foi desenvolvido por TOTTENHAM (1979), que também apresentou uma formulação integral para cascas abatidas.

A formulação direta para placas foi simultaneamente introduzida por BEZINE (1978a), (1978b) e STERN (1979), (1983). Bezine apresentou uma formulação integral usando elementos constantes e seus resultados se restringiram ao estudo de cargas concentradas. Stern desenvolveu uma formulação integral geral, mas não levou em conta a possibilidade da descontinuidade das condições de contorno nos nós de canto.

GOU-SHU (1986), HARTMANN \& ZOTEMANTEL (1986), adotaram esquema de interpolação hermitiana para a flecha e discutiram o tratamento das integrais de domínio, a consideração de vínculos no domínio e as singularidades que ocorrem na formulação direta via teoria de Kirchhoff. 
Adotando ainda as hipóteses de Kirchhoff, PAIVA (1987) utilizou várias alternativas para análise de placas, ora usando equações integrais para flecha e sua derivada primeira na direção normal ao contorno, ora usando duas equações de flecha para dois pontos singulares, um no contorno e outro fora do domínio. Esta análise foi estendida a placas combinados com vigas e pilares.

ABDEL-AKHER \& HARTLEY (1988), (1989a), (1989b) estudaram basicamente os problemas envolvendo as singularidades que aparecem nos integrandos interno.

Segundo SILVA (1996), a eficiência e as vantagens do Método dos Elementos de Contorno para análise de placas, baseada na Teoria clássica, enfatizadas por HARTMANN (1988), chega a superar o método dos elementos finitos para este tipo de problema.

Vários trabalhos foram publicados nos últimos tempos tratando de placas finas como PILTNER \& TAYLOR (1989), KATSIKADELIS \& ARMENÀKAS (1984), SAPOUNTZAKIS \& KATSIKADELIS (1991), VITOORAPORN \& MOSHAIOV (1989).

O equacionamento de placas apoiadas sobre base elástica foi inicialmente abordado por TOTTENHAM (1979), tendo como base as hipóteses de Winkler e Kirchhoff. Nesta mesma linha surgiram os trabalhos de KATSIKADELIS \& ARMENÀKAS (1984a), (1984b), COSTA JR.\& BREBBIA (1985a), (1985b), (1986), BEZINE (1988) e SILVA(1988).

KATSIKADELIS \& KALLIVOKAS (1986), (1988), retomando o estudo de placas sobre base elástica, adotou o modelo biparamétrico de Pasternak para simular a ligação solo-placa. KATSIKADELIS (1991) introduziu na formulação do problema a ocorrência de grandes deslocamentos, tendo como base as equações de Von Kármán, permitindo comportamento não linear entre o deslocamento e a resposta do solo.

Outro problema importante é a análise de placas com não-linearidade geométrica devido à ocorrência de grandes deslocamentos. Este assunto, de grande interesse para a engenharia de estruturas, foi tratado por TANAKA (1982), KAIYA \& SAWAKI (1984) e YE \& LIN (1985). O trabalho de TANAKA (1982) analisou placas finas elásticas com grandes deslocamentos e apresentou uma formulação 
integral e incremental equivalente às equações de Von Kármán, enquanto que o trabalho de KAMIYA \& SAWAKI (1984) baseou-se na equação de Bergar.

Podem-se citar outros trabalhos nesta área como os divulgados por SAWAKI et alii (1989), KATSIKADELIS \& NERANTZAKI (1988), KAMIYA et alii (1982) e KAMIYA (1989).

O problema envolvendo vibração livre e forçada em placas finas elásticas foi apresentado por PROVIDAKIS \& BESKOS (1989). AKKARI \& HUTCHINSON (1988) além de estudarem vibrações em placas finas, estenderam suas análises, contemplando placas espessas.

Uma análise elastodinâmica de placas, com interação solo-estrutura é feita em BARRETTO (1995) onde tanto a placa quanto o solo são analisados via o MEC.

A não-linearidade física em placas, envolvendo deformação lenta, plasticidade e fratura, foi objetivo de muitos estudos realizados por MUKERJEE \& MORJARIA et alii (1989), (1981) e MORJARIA et alii (1981a), (1981b). A análise do comportamento elastoplástico de placas finas via Kirchhoff foi desenvolvida por MOSHAIOV \& VORUS (1986), usando um esquema de carregamento incremental, com a consideração de momentos fletores plásticos iniciais, calculado por processo iterativo. A placa é dividida em células internas, onde as componentes do momento plástico são admitidas constantes. RIBEIRO (1992) atuou nesta mesma área considerando campos de momentos iniciais no domínio da placa, permitindo-se com isto, a análise de efeitos de gradiente de temperatura, além da consideração do comportamento não-linear do material. Também nesta linha de pesquisa, tem-se CHUEIRI (1994) que tratou da análise elastoplástica de placas, com as hipóteses da Teoria de Kirchhoff.

A instabilidade de placas sujeitas à cargas no seu próprio plano foi analisada por COSTA JR. (1985) e também por BEZINE et alii (1985), utilizando-se células internas para o cálculo da integração de domínio.

A análise de placas através da Teoria de Reissner foi inicialmente tratada por WEEËN (1982a), (1982b). KARAM (1986), usando como referência os trabalhos de Weeën, demonstrou a eficiência do método, através de vários exemplos de placas isotrópicas em regime elástico linear. RIBEIRO \& VENTURINI (1994), seguindo a 
formulação de WEEËN (1982a), (1982b), tomavam os pontos das cargas fora do domínio, evitando com isto algumas singularidades. Os trabalhos de BARCELOS \& SILVA (1987) e WESTPHAL \& BARCELOS (1989) abordaram também a flexão de placas usando as hipóteses de Reissner, identificando funções livres e funções essenciais como componentes da solução fundamental. A não-linearidade geométrica devido a ocorrência de grandes deslocamentos, analisada sob a teoria de Reissner, foi formulada por XIAO-YAN et alii (1990).

A solução de placas retangulares com variação de espessura tem sido de interesse de muitos investigadores, durante as últimas décadas, e em diferentes áreas, tais como engenharia civil, engenharia aeroespacial, em projetos de máquinas e em estruturas resistente a terremotos. Em caso de placas com espessura variável, a equação diferencial que governa este tipo de problema tem vários coeficientes, e este fato aumenta a dificuldade de solução. Há solução de placas circulares e anelares com variação da espessura ao longo do raio, sujeitas a carregamentos assimétricos, bem como placas retangulares com variação da espessura em apenas uma direção. Neste âmbito de estudo pode-se citar: TIMOSHENKO \& WOINOWSKY-KRIEGER (1959), CHEN (1976), BARES (1979), FERTIS \& MIJATOV (1989).

Um perfil com espessura variável e arbitrário só pode ser tratado via método numérico, tal como Método dos Elementos Finitos-MEF. Embora haja bastante aplicação do método dos elementos de contorno para análise de flexão de placas com espessura constante, como já foi citado anteriormente, este método não tem sido empregado com muita freqüência para análise de placas com espessura variável. Isso é devido a não possibilidade de obtenção da solução fundamental da equação que governa o problema. Pode-se citar alguns autores que trabalharam nesta área como: SAPOUNTZAKIS \& KATSIKADELIS (1991), GOSPODINOV (1988), DETO et alii (1988). 


\subsection{Objetivos}

O presente trabalho tem a finalidade de explanar o assunto de placas delgadas com espessura constante e variável baseada na teoria de Kirchhoff, utilizando a formulação direta do Método dos Elementos de Contorno (MEC).

O MEC tem sido bastante utilizado para análise de flexão de placas com espessura constante, obtendo-se bons resultados tanto para deslocamentos quanto para esforços.

Com a implementação da variação de espessura em placas, este trabalho dará base para desenvoivimento de análise de pavimento de edifício, tabuieiro de pontes, e etc., sendo suficiente a imposição de condições internas ao elemento de placa e associação com outros elementos estruturais através do acoplamento com o Método dos Elementos Finitos e da utilização de sub-regiões onde a espessura da laje é constante. No tratamento da integral de domínio do problema de espessura variável será necessário da utilização de aproximações definidas nas células internas.

Para atingir tais objetivos será necessário a implementação em microcomputador de toda a formulação requerida pelo método. A linguagem de programação adotada tem como compilador o FORTRAN PowerStation versão 4.0.

\subsection{Conteúdo do Trabalho}

No Capítulo 2, são apresentados alguns conceitos da teoria da elasticidade para melhor entendimento do assunto e apresenta também a teoria de placas, destacando-se os conceitos básicos e as equações da teoria de Kirchhoff para a flexão de placas, bem como as soluções fundamentais dos deslocamentos e esforços de placa, que são utilizados na formulação.

No Capítulo 3, determinam-se as equações integrais relativas aos deslocamentos para pontos do domínio e do contorno, assim como, faz-se a transformação das integrais de domínio, relativas ao carregamento, em integrais sobre 
o contorno da região carregada . Estas equações integrais são obtidas tanto para problema de flexão de placa com espessura constante quanto para o de variável.

No Capítulo 4, as equações integrais são discretizadas com a utilização de funções aproximadoras sobre o contorno, representando deslocamentos e esforços. Transformam-se, assim, as equações integrais em equações algébricas lineares.

No Capítulo 5, são apresentados alguns resultados que serão processados pelo sistema e comparados com alguns exemplos obtidos analiticamente. Neste mesmo capítulo são feitas as conclusões. 


\section{RELAÇÖES BÁSICAS: TEORIA DA ELASTICIDADE E PLACAS}

\subsection{Introdução}

Neste capítulo serão abordados as equações fundamentais para descrever o comporiamento de um sólido através da teoria da elasticidade, tais equações são classificadas em três categorias de equações: relação deformação-deslocamento; leis constitutivas; condições de equilibrio. Estas relações são muitas vezes expressas como equações diferenciais locais, podendo também ser escritas na forma integral.

Será discutida também a teoria de placas, mais especificamente a teoria de Kirchhoff.

\subsection{Relação Deformação-Deslocamento}

Quando a posição relativa entre dois pontos quaisquer num corpo contínuo é alterada, é dito que o corpo sofreu deformação. Se a distância entre qualquer par de ponto no corpo permanece constante durante o movimento, o corpo é dito rígido. $\mathrm{O}$ movimento de corpo rígido é caracterizado apenas pela translação e rotação.

No sistema de coordenadas cartesianas adotado, as três componentes, $\mathbf{u}_{\mathbf{i}}, \mathbf{u}_{2}$, $\mathbf{u}_{3}$ ou $\mathbf{u}, \mathbf{v}, w$, do vetor deslocamento no ponto do sólido são mutuamente ortogonais e são tomadas positivas seguindo orientação dos eixos de coordenadas.

Considere dois pontos $\mathbf{A}$ e $\mathbf{B}$ no sólido tridimensional, distantes entre si ds (infinitesimal). Como resultado da deformação, o ponto $\mathbf{A}$ move para $\mathbf{A}$ ' e o ponto $\mathbf{B}$ 
para B'. Através de manipulações algébricas, obtém-se o tensor de deformações dado por:

$$
\varepsilon_{\mathrm{ik}}=\frac{1}{2} \cdot\left(\mathrm{u}_{\mathrm{i}, \mathrm{k}}+\mathrm{u}_{\mathrm{k}, \mathrm{i}}+\mathrm{u}_{\ell, \mathrm{i}} \cdot \mathrm{u}_{\ell, \mathrm{k}}\right) \quad(\mathrm{i}, \mathrm{k}, \ell=1,2,3)
$$

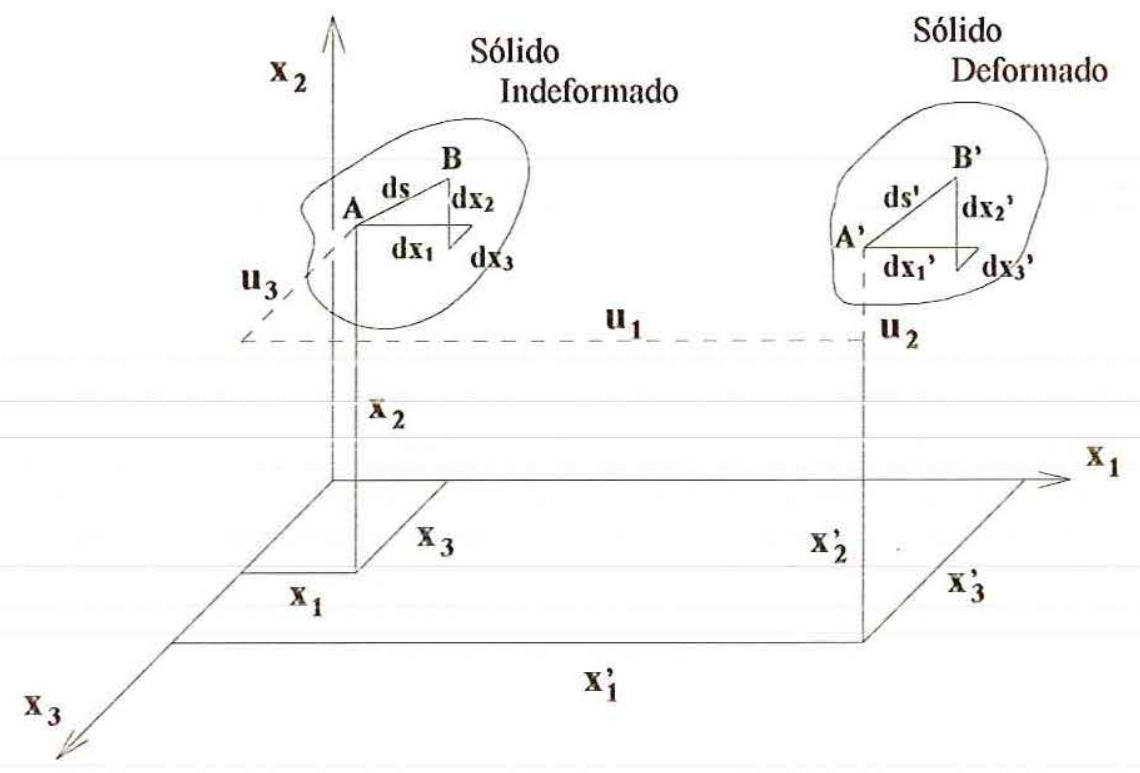

FIGURA 2.1 - Deformação de um Sólido Tridimensional

O tensor $\varepsilon_{i k}$ é conhecido como tensor de deformação de Green, embora seja usualmente considerado ter sido introduzido por Green e Saint-Venant.

Estas deformações são expressas em termo das coordenadas $x_{1}, x_{2}, x_{3}$ no estado indeformado, isto é, as chamadas coordenadas Lagrangeanas. Alternativamente, $\mathbf{d s} \mathbf{s}^{2}-\mathbf{d \mathbf { s } ^ { 2 }}$ pode ser escrito em termos de coordenadas no estado deformado, isto é, coordenadas Eulerianas $x_{1}{ }^{3}, x_{2}{ }^{3}, x_{3}{ }^{3}$. O tensor obtido a partir destas coordenadas é conhecido como tensor de deformação de Almansi. Este tensor de deformação foi proposto por Almansi e Hamel.

A descrição Lagrangeana é preferida em análise estrutural porque é mais fácil de caracterizar um corpo indeformado do que um deformado. 
Em muitos casos de importância prática, as deformações nas estruturas são pequenas o bastante para que o termo quadrático da eq.(2.1) não tenha nenhuma contribuição significativa e, portanto, negligenciá-lo é justificável. A simplificação desta teoria resulta em expressões lineares, tais como:

$$
\varepsilon_{i k}=\frac{1}{2} \cdot\left(u_{i, k}+u_{k, i}\right) \quad(i, k=1,2,3)
$$

\subsection{Lei Constitutiva}

A lei constitutiva ou equação constitutiva proporciona uma relação entre a tensão $(\sigma)$ e a deformação $(\varepsilon)$. Na região elástica linear de um sólido homogêneo, a lei constitutiva é usualmente chamada de Lei de Hooke.

A generalidade das equações da lei de Hooke é devida a Navier-Stokes. Daí, as seis componentes de tensão, em qualquer ponto do sólido elástico, serão relacionadas com as seis componentes de deformação pelas equações a seguir:

$$
\sigma_{\mathrm{ij}}=\mathbf{C}_{\mathrm{ijk} \ell} \cdot \varepsilon_{\mathrm{ij}}
$$

onde o tensor de quarta ordem $\mathbf{C}_{\mathrm{ijk} \text { t }}$ é formado por componentes que contêm constantes elásticas do material.

O coeficiente $\mathbf{C}_{\mathrm{ijk} \ell}$ da eq.(2.3), em geral, variam de ponto a ponto do meio contínuo. Entretanto, sendo admitidas as hipóteses de meio elástico, homogêneo e isótropo, $\mathbf{C}_{\mathbf{i j k} \ell}$ pode ser considerado independente da posição do ponto e, com isso, reduz-se o número de constantes elásticas para dois valores $(\mathbf{E}, v)$, podendo-se então rescrever a eq.(2.3) como: 


$$
\sigma_{\mathrm{ij}}=\frac{\mathbf{E} \cdot v}{(1+v) \cdot(1-2 \cdot v)} \cdot \delta_{\mathrm{ij}} \cdot \varepsilon_{\mathrm{kk}}+\frac{\mathbf{E}}{(1+v)} \cdot \varepsilon_{\mathrm{ij}}=\lambda \cdot \delta_{\mathrm{ij}} \cdot \varepsilon_{\mathrm{kk}}+2 \cdot \mu \cdot \varepsilon_{\mathrm{ij}}
$$

A forma inversa da eq.(2.4) é dada por:

$$
\varepsilon_{\mathrm{ij}}=\frac{1+v}{\mathbf{E}} \cdot \sigma_{\mathrm{ij}}-\frac{v}{\mathbf{E}} \cdot \delta_{\mathrm{ij}} \cdot \sigma_{\mathrm{kk}}
$$

onde:

- $\delta_{\mathrm{ij}}$ é o tensor simétrico de segunda ordem mais simples conhecido como delta de Kronecker ou tensor de substituição, definido por:

$$
\delta_{i j}=\left\{\begin{array}{lll}
1 & \text { se } i=j \\
0 & \text { se } i \neq j
\end{array}\right.
$$

- $\lambda=\frac{\mathbf{E} \cdot v}{(1+v) \cdot(1-2 \cdot v)}$ e $\mu=\mathbf{G}=\frac{\mathbf{E}}{2 \cdot(1+v)}$ são as constantes de Lamé;

- $v$ - coeficiente de Poisson;

- $\mathbf{E}$ - módulo de elasticidade longitudinal do material;

- $\mathbf{G}$ - módulo de elasticidade transversal do material, que é função de $\mathbf{E}$ e $\mathbf{v}$.

\subsection{Condições de Equilíbrio}

A descrição do equilibrio em qualquer ponto do corpo é caracterizada por equações diferenciais envolvendo tensões e forças internas (volumétricas, de massa), tais como a gravitacional. 

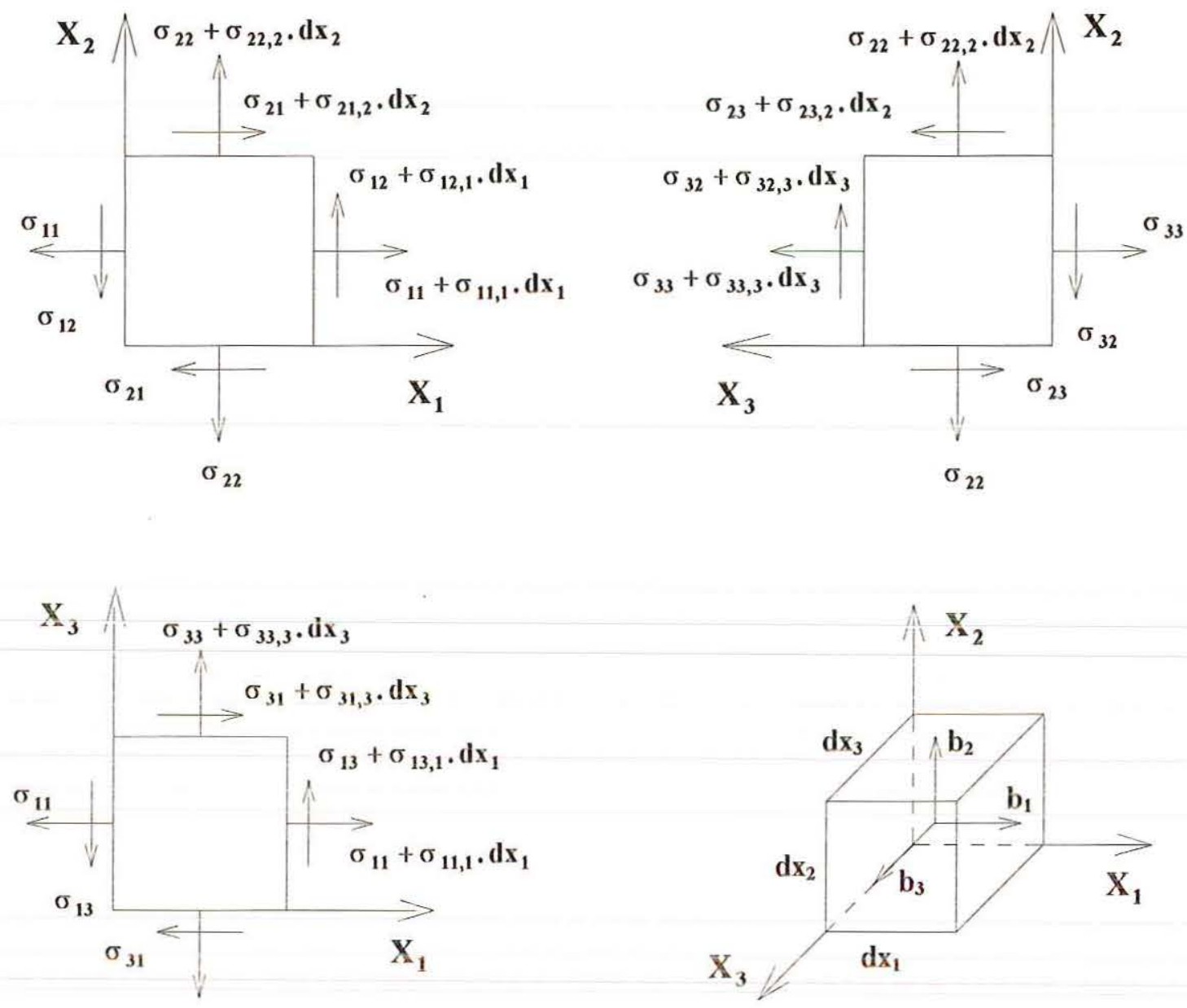

$\sigma_{33}$

FIGURẢ 2.2 - Campo de Tensões em Três Dimensões.

Desde que o corpo como um todo deva estar em equilíbrio, o elemento acima considerado, figura 2.2, deve também estar em equilíbrio considerando-se as tensões que agem em suas faces e as forças volumétricas. Escrevendo-se as equações de equilíbrio nas direções $\mathbf{x}_{1}, \mathbf{x}_{2}$ e $\mathbf{x}_{3}$, obtém-se:

$$
\sigma_{i j, j}+b_{i}=0 \quad(i, j=1,2,3)
$$

onde $\mathbf{b}_{\mathbf{i}}(\mathbf{i}=\mathbf{1}, \mathbf{2}, \mathbf{3})$ são as forças volumétricas. 


\subsection{Forças de Superfície}

Dadas as seis componentes de tensão em um ponto (levando-se em conta a simetria do tensor) referente a algum sistema de coordenadas $\left(\mathbf{X}_{1}, \mathbf{X}_{2}\right.$ e $\left.\mathbf{X}_{3}\right)$, é possível determinar as tensões agindo em qualquer plano passando por este ponto. Isto pode ser feito por consideração do equilíbrio estático de um tetraedro infinitesimal formado por este plano e pelos planos cartesianos como mostra a figura $2.3 \mathrm{a}$. Nesta figura, são mostradas as tensões agindo nas faces contidas nos três planos cartesianos. Estas tensões são admitidas conhecidas e pretende-se encontrar as tensões agindo no plano ABC cuja norma $\mathrm{ON}$ tem co-senos diretores $\ell_{1}, \ell_{2}$ e $\ell_{3}$. Estas tensões são conhecidas como Forças de Superficie (Tractions) e são obtidas através do equilibrio do tetraedro, resultando na seguinte expressão:

$$
\mathbf{p}_{\mathrm{i}}=\sigma_{\mathrm{ij}} \ell_{\mathrm{j}} \quad(\mathbf{i}, \mathbf{j}=\mathbf{1 , 2 , 3 )}
$$

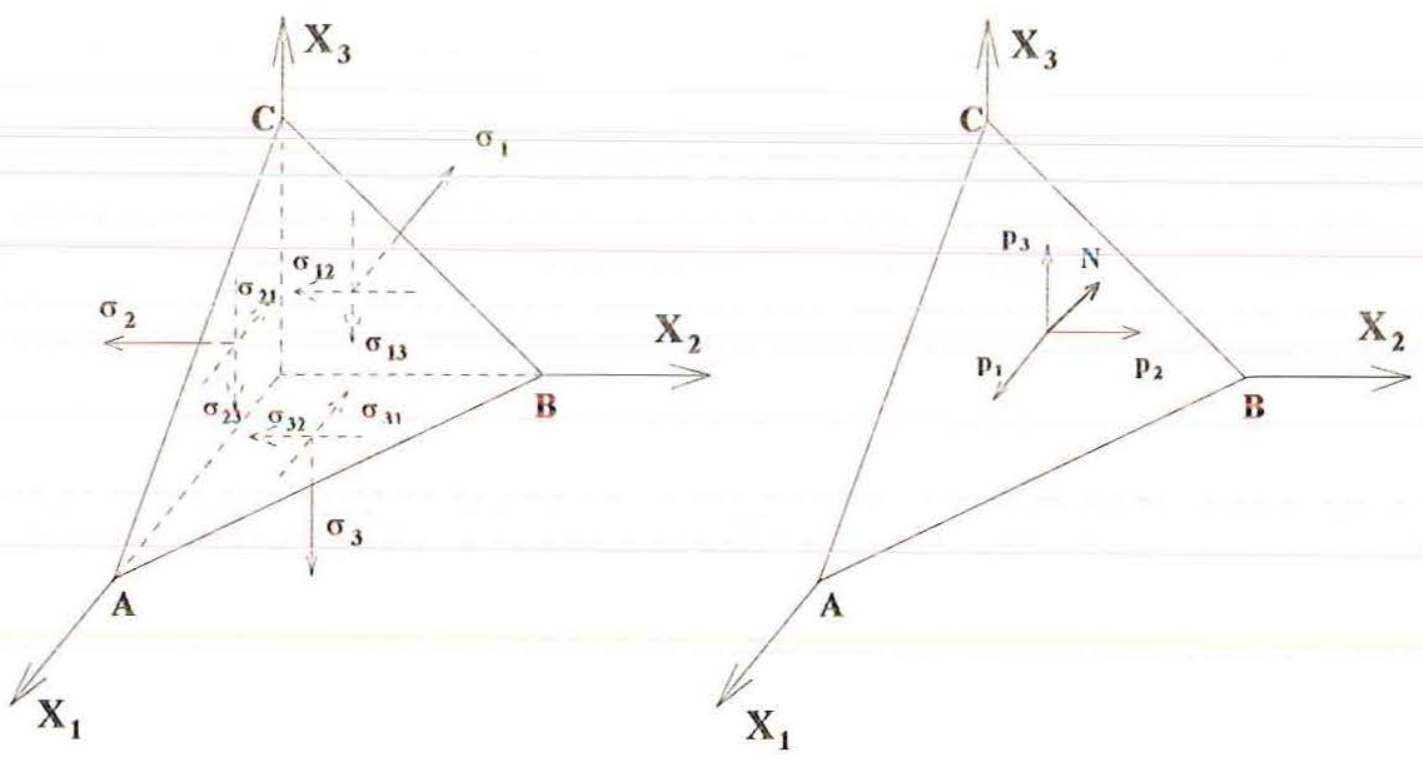

(a) Tensões

(b) Forças de Superfície

FIGURA 2.3 - Tensões e Forças de Superfície num Tetraedro Infinitesimal. 


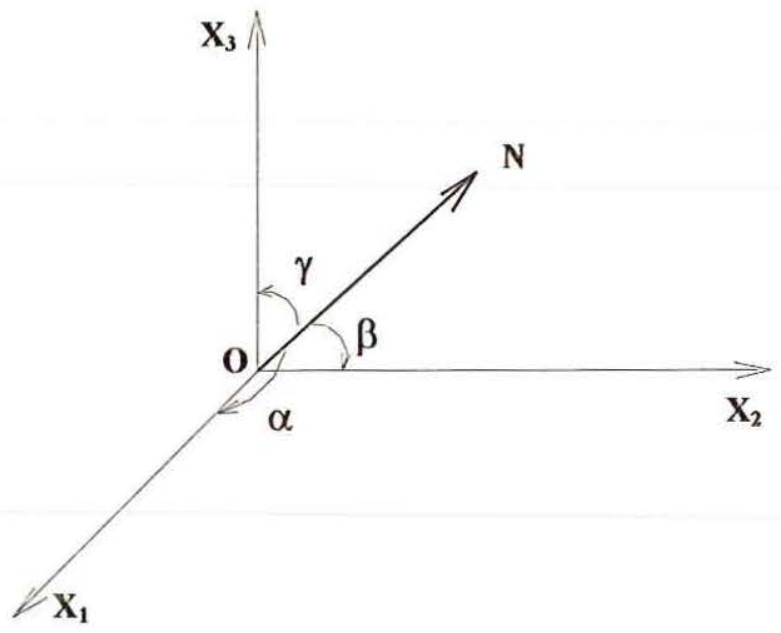

$$
\begin{aligned}
& \ell_{1}=\cos \left(\mathrm{N}, \mathrm{x}_{1}\right)=\cos (\alpha) \\
& \ell_{2}=\cos \left(\mathrm{N}, \mathrm{x}_{2}\right)=\cos (\beta) \\
& \ell_{3}=\cos \left(\mathrm{N}, \mathrm{x}_{3}\right)=\cos (\gamma)
\end{aligned}
$$

FIGURA 2.4 - Co-senos Diretores do Vetor ON.

Para o caso bidimensional, figura 2.5, as componentes de tensão na superficie de um ponto devem estar em equilibrio com as forças aplicadas na superfície. As equações de equilíbrio são obtidas como no caso tridimensional, resultando na eq. (2.7), $\operatorname{com} \mathbf{i}, \mathbf{j}=\mathbf{1 , 2}$.

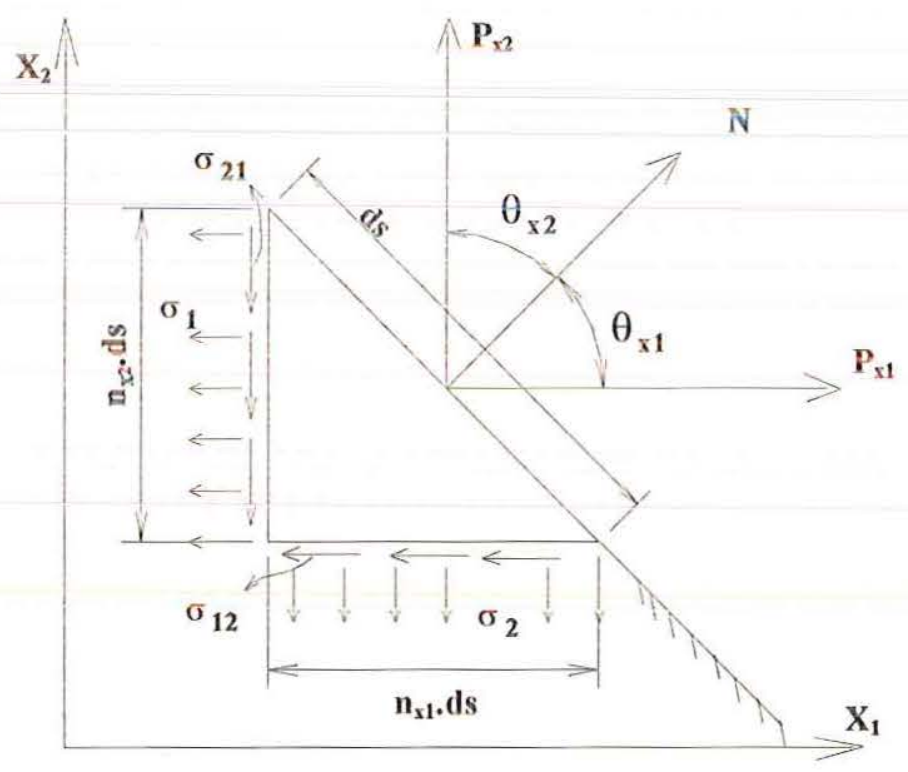

FIGURA 2.5 - Tensões e Forças de Superficie no Caso Bidimensional. 


\subsection{Elementos Estruturais}

Os elementos estruturais têm a finalidade de transferência de cargas e, conforme a relação entre suas dimensões, são classificados como:

a) lineares (barra) - duas dimensões pequenas quando comparada com a terceira dimensão; como exemplo, pode-se citar:

- vigas;

- elementos estruturais de pórtico;

- elementos estruturais de treliça;

- elementos estruturais de grelha;

- elementos estruturais de arco.

b) de superfície (laminares) - uma dimensão pequena quando comparada com as outras duas dimensões; como exemplo, pode-se citar:

- placas - superfície média plana com cargas aplicadas normalmente a este plano;

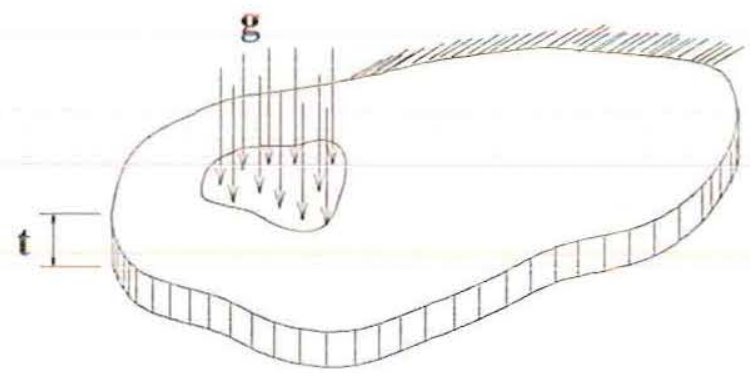

FIGURA 2.6 - Exemplo de Placa. 
- chapas - superfície média plana com forças externas aplicadas neste plano;

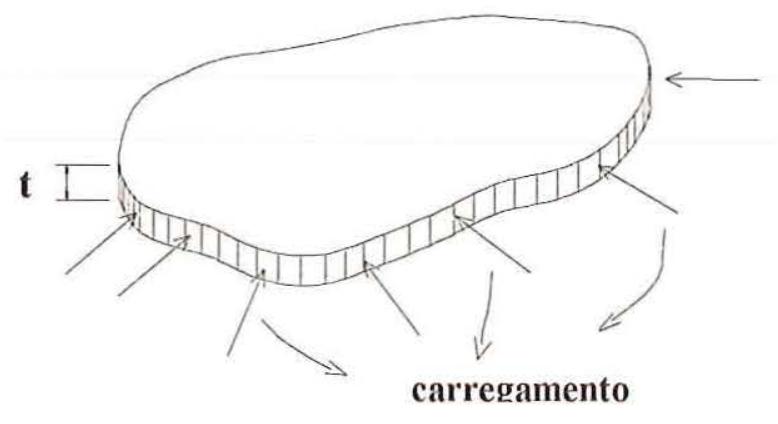

FIGURA 2.7 - Exemplo de Chapa.

- cascas - são estruturas com a superficie média curva;

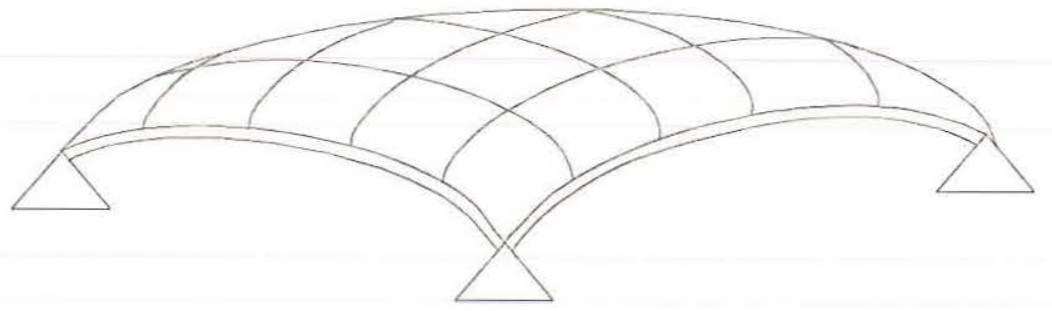

FIGURA 2.8 - Exemplo de Casca.

c) tridimensionais (blocos) - as três dimensões têm a mesma ordem de grandeza; como exemplo, pode-se citar:

- bloco de fundação, sapatas.

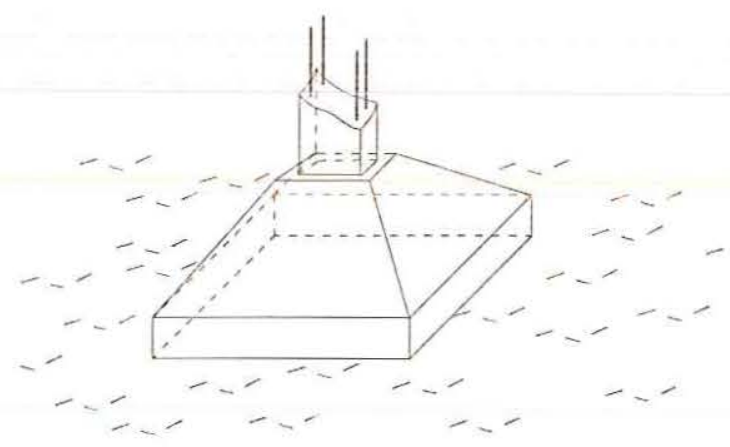

FIGURA 2.9 - Exemplo de Sapata. 


\subsection{Placas}

A placa é definida usualmente como um corpo limitado por duas superfícies geralmente planas. A superficie neutra é equidistante às duas superfícies limites, cuja distância entre estas superficies, chamada espessura (que pode ser variável), é pequena se comparada com as outras duas dimensões, isto é, $t / a<<1$, figura 2.10 .

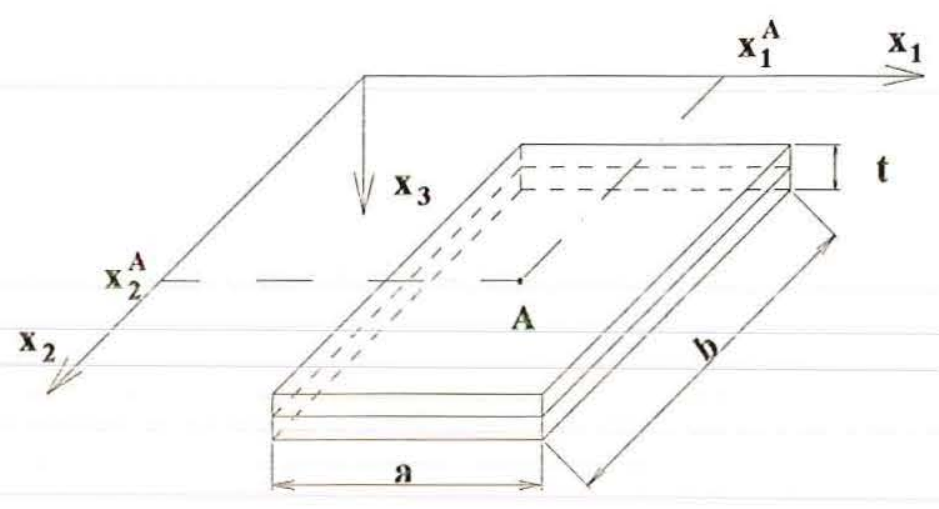

FIGURA 2.10 - Placa Delgada de Espessura t.

De acordo com o material do qual é constituída a placa, ela pode ser classificada como:

a) anisótropa - cujas propriedades são diferentes em qualquer direção;

b) ortótropa - cujas propriedades são diferentes em duas direções ortogonais;

c) isótropa - cujas propriedades são as mesmas em qualquer direção.

Dependendo da relação (t/a) entre a espessura (t) e a menor dimensão (a) medida no plano médio, a placa pode ser classificada como:
a) muito delgada: $\frac{t}{a}<\frac{1}{80}$;
b) delgada: $\frac{1}{80} \leq \frac{t}{a} \leq \frac{1}{5}$;
c) espessa: $\frac{\mathbf{t}}{\mathrm{a}}>\frac{1}{\mathbf{5}}$. 
Várias hipóteses têm sido estabelecidas para teoria de placas. Dentre elas pode-se citar: Teoria Reissner, Reissner-Mindlin e a de Kirchhoff.

O mais adequado ponto de partida para a análise de placas espessas ou moderadamente espessas parece ser a teoria na qual as hipóteses básicas da deformação por tensão cisalhante são consideradas mas, com certa simplificação.

Primeiramente, REISSNER (1944) propôs que as rotações das normais ao plano da superficie média nos planos $\mathbf{x}_{1} \mathbf{x}_{3}$ e $\mathbf{x}_{2} \mathbf{x}_{3}$ poderiam ser introduzidas como variáveis independentes, sendo por isso uma teoria mais refinada. MINDLIN (1951) simplificou a suposição de Reissner, admitindo que as seções normais ao plano da superfície média antes da deformação permanecem plana, mas não necessariamente normais à superfície média após a deformação e desprezando a tensão normal à superfície média. A teoria de Kirchhoff admite também que as seções normais ao plano da superficie média antes da deformação permanecem retas e, além disso, que permanecem normais à superficie média após a deformação.

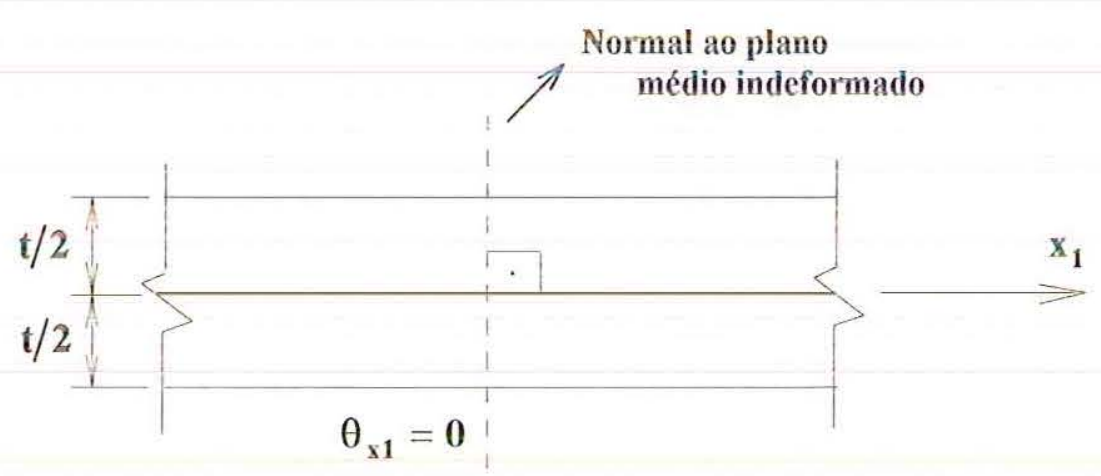

FIGURA 2.11 - Plano Médio Indeformado da Placa. 


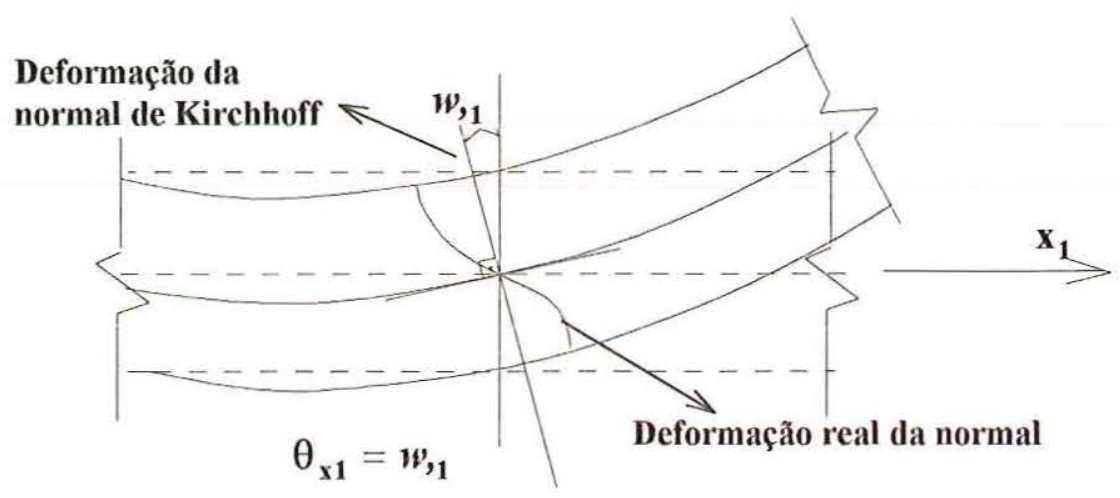

FIGURA 2.12 - Plano Médio Deformado Conforme Teoria de Kirchhoff.

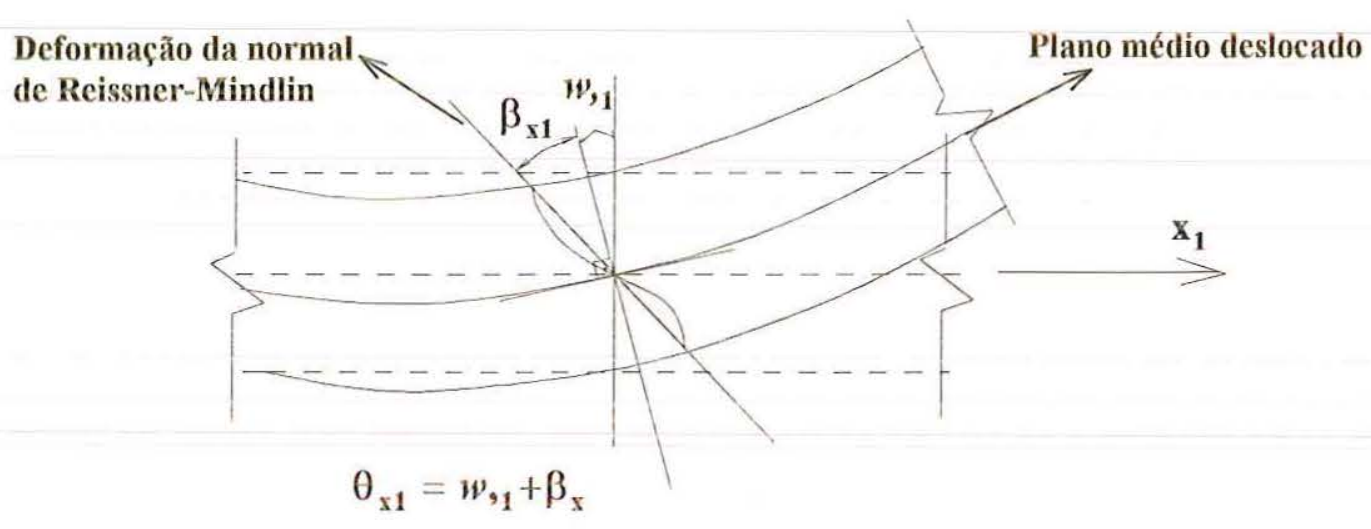

FIGURA 2.13 - Plano Médio Deformado Conforme Teoria de ReissnerMindlin.

Neste trabalho, serão consideradas placas com espessura constante ou variável, submetidas a carregamento transversal e ortogonal ao plano médio, baseando-se na teoria de Kirchhoff, restrita a placas delgadas por desprezar o efeito das deformações cisalhantes transversais. 


\subsection{Hipóteses e Relações Básicas da Teoria de Kirchhoff}

Em vista das limitações impostas, a Teoria de Kirchhoff leva a uma simplificação do problema. Para a determinação das relações e equações diferenciais básicas das placas delgadas, são necessárias as seguintes hipóteses:

1 - O material da placa é considerado elástico-linear, homogêneo e isótropo, obedecendo à lei de Hooke;

2 - Os deslocamentos transversais são pequenos, quando comparados com a espessura $i$ da placa;

3 - Não há deformação no plano médio da placa (superfície neutra);

4 - Dada uma seção plana e perpendicular à superfície média, esta permanece plana, perpendicular e indeformada em relação àquela superfície após a flexão.

A partir das simplificações admitidas nas hipóteses, pode-se determinar as relações e equações fundamentais da Teoria de Kirchhoff para placas delgadas e isótropas.

\subsubsection{Campo de Deslocamento}

Os deslocamentos $\mathbf{u}_{1}, \mathbf{u}_{2}$ e $\mathbf{u}_{3}$ de um ponto qualquer da placa são expressos pela deflexão $w$ e pelas rotações $\theta_{\mathrm{X}_{1}}$ e $\theta_{\mathrm{X}_{2}}$ da superfície média, que coincide com o plano $\mathbf{x}_{1}-\mathbf{x}_{2}$ do sistema de coordenadas. Na figura 2.14 são indicados os deslocamentos positivos. 


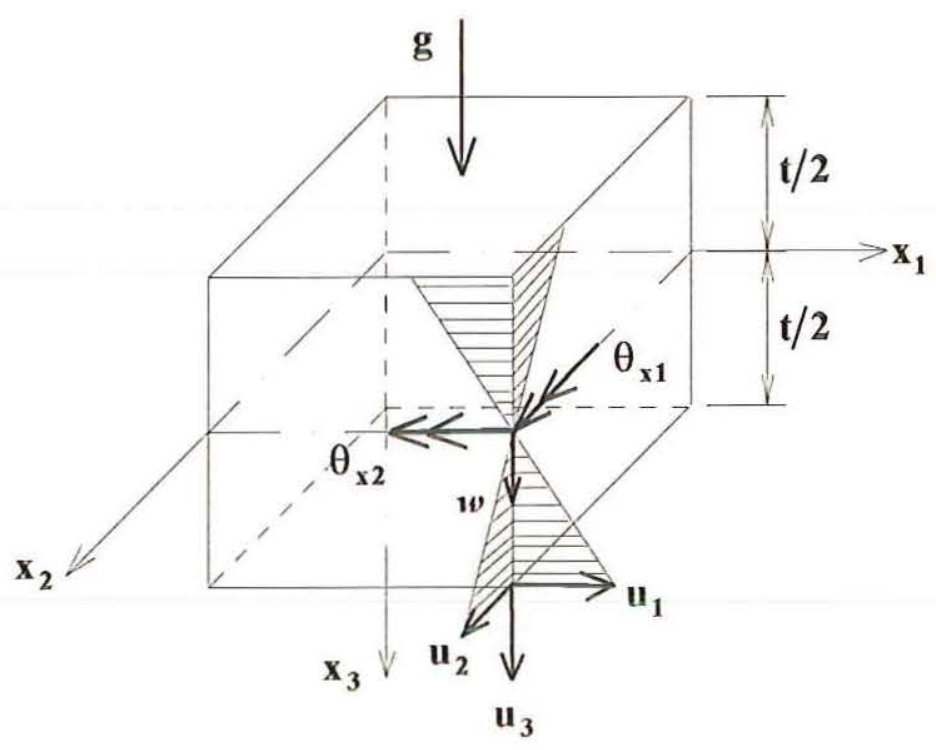

FIGURA 2.14 - Deslocamento Positivo de um Ponto Qualquer.

Das hipóteses básicas apresentadas anteriormente, pode-se concluir em relação as deformações, que:

$$
\varepsilon_{\mathrm{i} 3}=0 \quad(\mathrm{i}=1,2,3)
$$

Sendo $\varepsilon_{33}=0$, ao integrar $\frac{\partial \mathbf{u}_{3}}{\partial \mathbf{x}_{3}}=0$, obtém-se:

$$
u_{3}=f\left(x_{1}, x_{2}\right)
$$

indicando que o deslocamento transversal ao plano médio da placa $\mathbf{u}_{3}$, não varia através da espessura da placa, isto é, não varia $\operatorname{com} \mathrm{x}_{3}$, sendo $f\left(\mathrm{x}_{1}, \mathrm{x}_{2}\right)$ a função que representa os deslocamentos transversais do plano médio.

Considerando-se a segunda e a quarta hipótese básica, vistas anteriormente, pode-se concluir que, dado um ponto $\mathbf{p}$ que dista de $\mathbf{x}_{3}$ da superfície média, após o carregamento, este ponto desloca-se na direção $\mathrm{x}_{1}$ de um valor, em módulo, igual a 
$\mathbf{x}_{3} \cdot \frac{\partial w}{\partial \mathbf{x}_{1}}$ e na direção $\mathbf{x}_{2}$, igual a $\mathbf{x}_{3} \cdot \frac{\partial w}{\partial \mathbf{x}_{2}}$, ver figura 2.15 . Logo, pode-se concluir que:

$$
\mathbf{u}_{\mathbf{i}}=-\mathbf{x}_{3}, w_{,} \quad(\mathbf{i}=1,2)
$$

Antes do carregamento

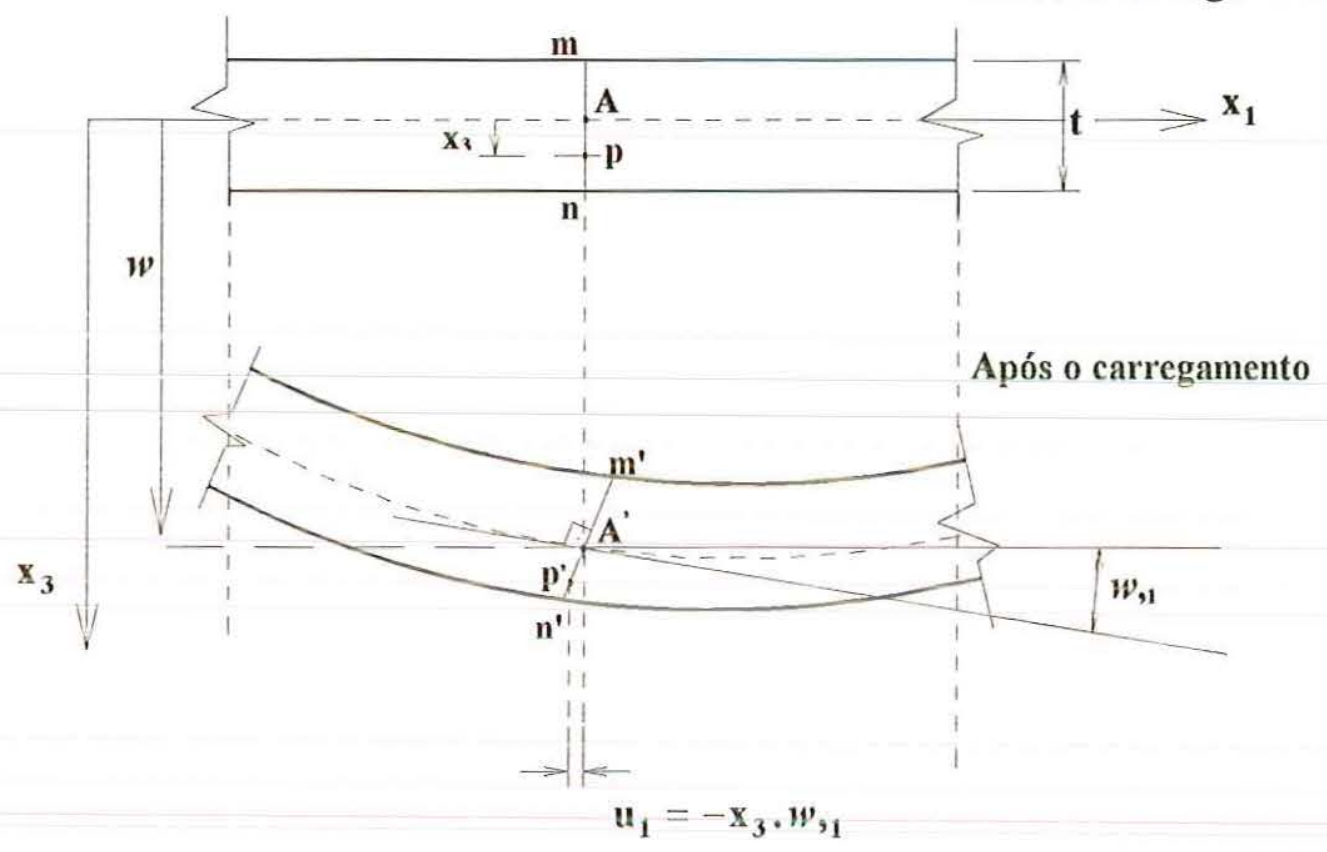

FIGURA 2.15 - Placa Antes e Após o Carregamento.

\subsubsection{Campo de Deformação}

Diferenciando-se a eq.(2.10), pode-se obter:

$$
\mathbf{u}_{\mathrm{i}, \mathrm{j}}=-\mathrm{x}_{3} \cdot w_{\mathbf{i j}_{\mathrm{j}}} \quad(\mathrm{i}, \mathrm{j}=1,2)
$$

Substituindo (2.11) na relação deformação-deslocamento, dada pela eq.(2.2), e ainda considerando o Teorema de Schwartz que diz que, em existindo uma função $w=\mathrm{f}(\mathrm{x}, \mathrm{y})$ e esta, assim como as suas derivadas parciais: $w_{11}, w, w_{2}, w, 12, w, 21$, forem contínuas num determinado intervalo, então a igualdade $w, w_{12}=w, 21$ 
verdadeira, logo a equação que relaciona deformação-deslocamento pode ser rescrita como:

$$
\varepsilon_{\mathrm{ij}}=-\mathrm{x}_{3} \cdot w,_{\mathrm{ij}} \quad(\mathrm{i}, \mathrm{j}=1,2)
$$

\subsubsection{Campo de Tensão}

As componentes de tensão $\sigma_{1}, \sigma_{2}$ e $\sigma_{12}$ da eq.(2.4) foram obtidas utilizando-se as leis constitutivas do material. Já as demais componentes $\sigma_{3}, \sigma_{13}$ e $\sigma_{23}$ não podem ser obtidas a partir das leis constitutivas, uma vez que elas estão relacionadas com as deformações $\varepsilon_{3}, \varepsilon_{13}$ e $\varepsilon_{23}$, as quais foram desprezadas como resultado das hipóteses básicas. Contudo, as equações de equilíbrio (2.6) permitirão que estas tensões sejam determinadas. Através de integrações dessas equações, podese obter:

$$
\begin{array}{r}
\sigma_{\mathrm{i} 3}=\frac{3 \cdot q_{\mathrm{i}}}{2 \cdot \mathrm{t}} \cdot\left[1-\left(\frac{2 \cdot \mathrm{x}_{3}}{\mathrm{t}}\right)^{2}\right] \quad(i=1,2) \\
\sigma_{3}=-\frac{3}{4} \cdot \mathrm{q}_{\mathrm{k}, \mathrm{k}} \cdot\left[-\frac{2}{3}+\frac{2 \cdot \mathrm{x}_{3}}{\mathrm{t}}-\frac{1}{3} \cdot\left(\frac{2 \cdot \mathrm{x}_{3}}{\mathrm{t}}\right)^{3}\right]
\end{array}
$$

onde: $q_{1}$ são as forças cortantes.

Pode-se observar que $\sigma_{13}$ e $\sigma_{23}$ variam parabolicamente sobre a espessura enquanto que $\sigma_{3}$ varia cubicamente, figura 2.16. Porém, essas tensões $\sigma_{\mathbf{i} 3}(\mathbf{i}=\mathbf{1}, \mathbf{2}, \mathbf{3})$ são pequenas quando comparadas com as tensões $\sigma_{\mathbf{i j}}(\mathbf{i}, \mathbf{j}=\mathbf{1}, \mathbf{2})$. 


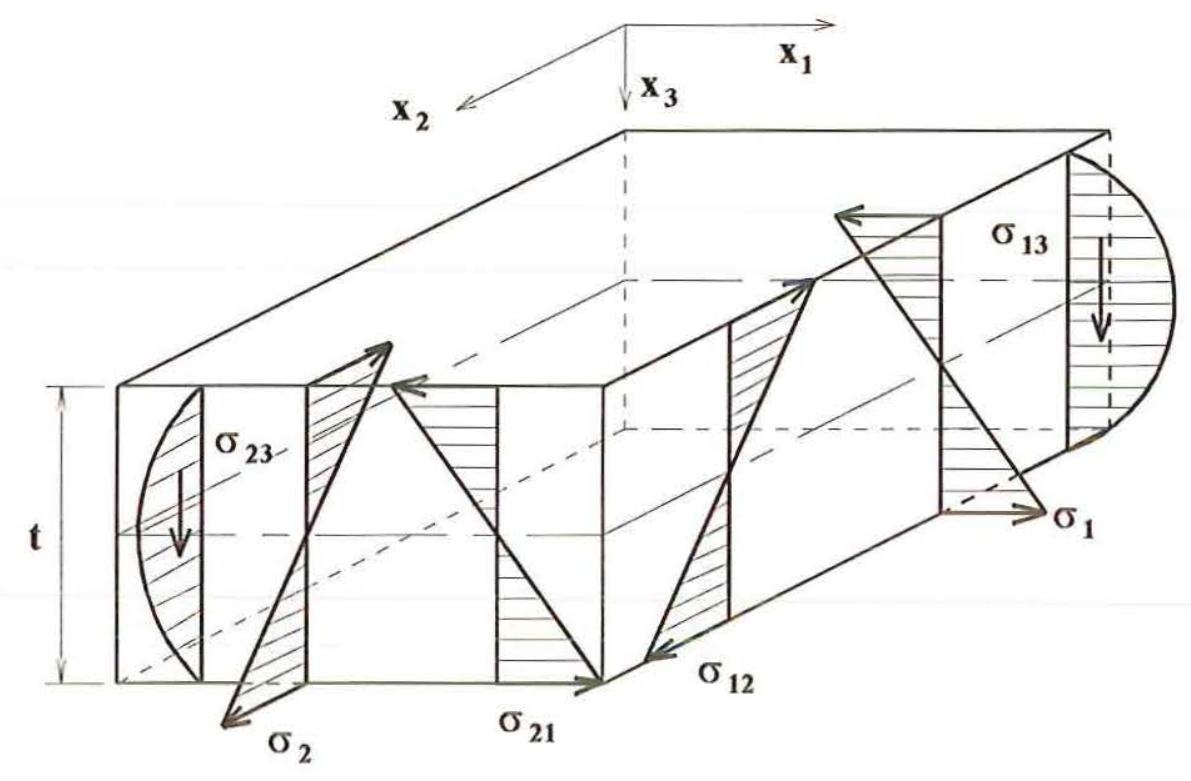

FIGURA 2.16 - Distribuição de Tensões.

Substituindo a eq.(2.12) na eq.(2.4), chega-se a:

$$
\sigma_{\mathrm{ij}}=-\frac{\mathbf{E} \cdot \mathbf{x}_{3}}{\left(1-v^{2}\right)}\left[v w_{9 \mathrm{kk}} \cdot \delta_{\mathrm{ij}}+(1-v), w_{9 \mathrm{ij}}\right] \quad(\mathbf{i}, \mathbf{j}, \mathbf{k}=\mathbf{1 , 2 )}
$$

Pode-se observar que $\sigma_{i j}$, da eq.(2.15), varia linearmente com a espessura da placa, figura 2.16 .

\subsubsection{Esforços de Placas}

Os esforços em um ponto qualquer da placa podem ser obtidos através dos resultados das componentes de tensão que atuam em um elemento infinitesimal $\left(\mathbf{d x}_{1}, \mathbf{d x}_{2}\right)$ de placa. Para isso, considere-se o elemento de placa representado na figura $2.17 \mathrm{a}$, onde são mostradas as componentes de tensão atuantes $\sigma_{\mathbf{i j}}$ nos seus sentidos positivos. 


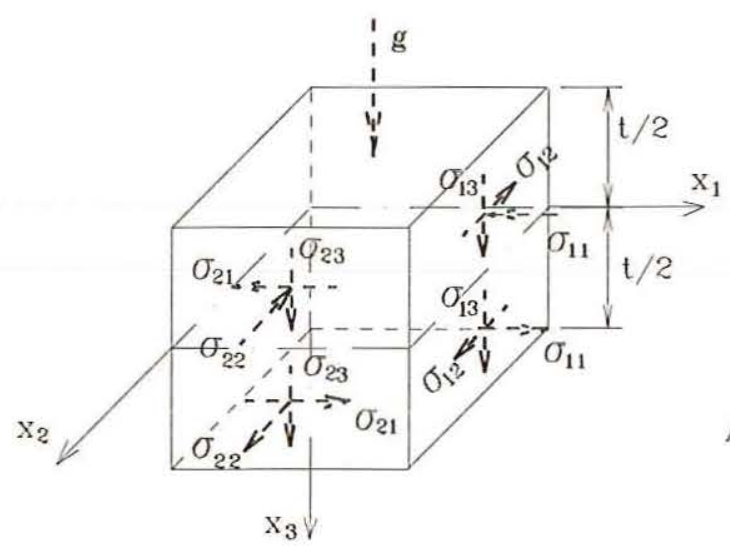

(a) Tensões

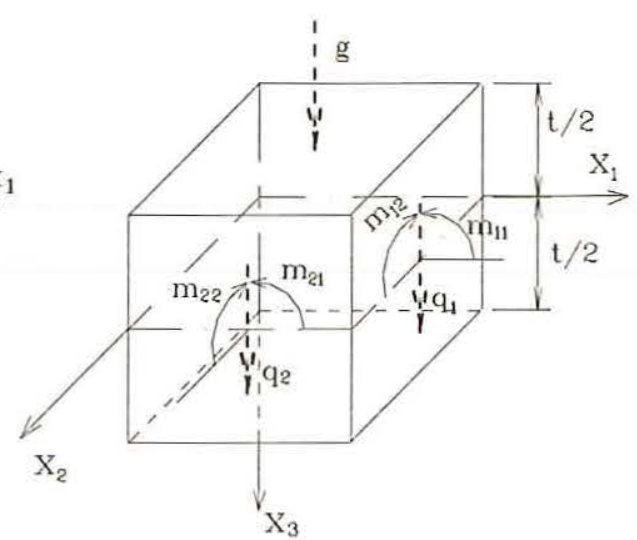

(b) Esforços

FIGURA 2.17 - Esforços Atuantes em um Elemento Infinitesimal de Placa.

Os esforços representados na figura $2.17 \mathrm{~b}$ serão dados pelas seguintes integrais sobre a espessura da placa:

$$
\begin{aligned}
m_{i j}=\int_{-t / 2}^{+t / 2} \sigma_{i j} \cdot x_{3} \cdot d x_{3} & (i, j=1,2) \\
q_{i}=\int_{-t / 2}^{+t / 2} \sigma_{i 3} \cdot d x_{3} & (i, j=1,2)
\end{aligned}
$$

que representam, respectivamente, os momentos e as forças corianies em um pontio qualquer da placa.

Substituindo-se a eq.(2.15) na eq.(2.16) e efetuando a integração, obtêm-se as equações dos momentos em termos dos deslocamentos transversais:

$$
\mathrm{m}_{\mathrm{ij}}=-\mathrm{D} \cdot\left[v \cdot \delta_{\mathrm{ij}} \cdot w_{\mathrm{sk}_{\mathrm{k}}}+(1-v), w_{\mathrm{ij}}\right] \quad(\mathrm{i}, \mathrm{j}, \mathrm{k}=1,2)
$$

sendo D a rigidez à flexão da placa dada por:

$$
D=\frac{E \cdot t^{3}}{12 .\left(1-v^{2}\right)}
$$




\subsubsection{Equação Diferencial de Placas}

Considerando-se o equilíbrio dos esforços no elemento diferencial de placa $\left(\mathbf{d x}_{\mathbf{1}} \cdot \mathbf{d} \mathbf{x}_{2}\right)$, figura 2.18 , sujeita a uma carga uniformemente distribuída $\mathbf{g}$, pode-se estabelecer o equilíbrio dos esforços deste elemento diferencial, chegando-se, após algumas operações algébricas, às equações de equilíbrio das forças verticais:

$$
q_{i, i}+g=0 \quad(i, j=1,2)
$$

e dos momentos:

$$
m_{j i, j}-q_{i}=0 \quad(i, j=1,2)
$$

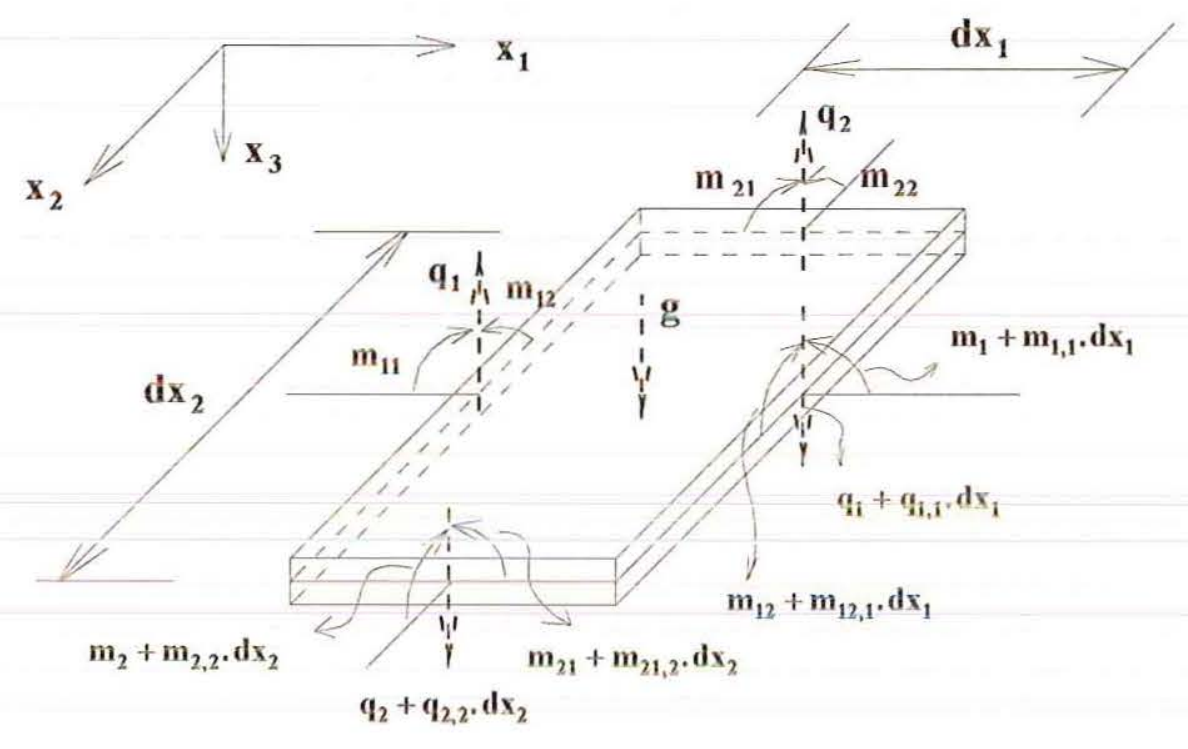

FIGURA 2.18 - Esforços em um Elemento Diferencial $\left(\mathbf{d x}_{\mathbf{1}} \cdot \mathbf{d} \mathbf{d x}_{\mathbf{2}}\right)$ de Placa.

Derivando-se a eq.(2.18), obtém-se:

$$
\mathbf{m}_{\mathrm{ij}, \mathrm{j}}=-\mathrm{D}\left[v \cdot \delta_{\mathrm{ij} \cdot} w_{9_{\mathrm{kkj}}}+(\mathbf{1}-v), w_{\mathrm{ijj}_{\mathrm{jj}}}\right] \quad(\mathbf{i}, \mathbf{j}, \mathbf{k}=\mathbf{1 , 2})
$$


e, utilizando-se da definição do tensor de substituição e do índice mudo, pode-se escrever a eq.(2.22) como:

$$
\mathbf{m}_{\mathrm{ij}, \mathrm{j}}=-\mathbf{D} . w_{i_{\mathrm{ijj}}}
$$

Substituindo-se a eq.(2.23) na eq.(2.21), resulta:

$$
\mathrm{q}_{\mathrm{i}}=-\mathrm{D} . \mathrm{w}_{\mathrm{jji}} \quad(\mathrm{i}, \mathrm{j}=1,2)
$$

Derivando-se a eq.(2.24) e substituindo-se na eq.(2.20) resulta em:

$$
w_{\text {iijj }}=\frac{\mathrm{g}}{\mathrm{D}} \quad(\mathrm{i}, \mathrm{j}=1,2)
$$

que é a equação diferencial de placas na notação indicial.

É possível também, obter a equação diferencial de placas em termos dos momentos, a partir das equações de equilíbrio (2.20) e (2.21), chegando-se a:

$$
\mathbf{m}_{\mathrm{ij}, \mathrm{ij}}+\mathbf{g}=\mathbf{0}
$$

\subsubsection{Esforço Cortante Equivalente}

Para a solução da equação diferencial de placas é necessário que sejam satisfeitas duas condições de contorno do problema. Nos problemas usuais, estas condições referem-se ao deslocamento transversal do plano médio, às suas rotações, às forças, momentos de flexão e momentos de torção dos pontos do contorno da placa.

Como a equação diferencial da placa é de quarta ordem, devem ser satisfeitas duas condições ao longo da borda, envolvendo apenas quatro variáveis do problema. KIRCHHOFF (1850), usando relação de energia, demonstrou que as condições de contorno relativas à força cortante $\mathbf{q}_{\mathbf{i}}(\mathbf{i}=\mathbf{1 , 2})$ e ao momento de torção $\mathbf{m}_{\mathbf{i j}}(\mathbf{i} \neq \mathbf{j}=\mathbf{1 , 2})$ devem ser agrupadas em uma única condição, relativa ao esforço $V_{i}(i=1,2)$, denominado força cortante equivalente ou força de Kirchhoff. De 
acordo com o princípio de St. Venant, esta substituição afeta a distribuição de tensões apenas na vizinhança do contorno.

Estaticamente, o momento de torção $\mathbf{m}_{\mathbf{i j}}(\mathbf{i} \neq \mathbf{j}=\mathbf{1 , 2})$ da figura $2.17 \mathrm{~b}$, pode ser representado por um binário de forças horizontais ou verticais, figura 2.19a. Considere dois elementos sucessivos de comprimento $\mathbf{d x}_{2}$ na borda $\mathbf{x}=\mathbf{a}$ como mostra a figura 2.19b. Conforme ilustra a figura $2.19 \mathrm{c}$, em um elemento, o momento volvente $\mathrm{m}_{12} \cdot \mathbf{d x}_{2}$ é substituído por um binário estaticamente equivalente com forças iguais a $\mathbf{m}_{12}$ separadas por $\mathbf{d x}_{2}$ e no elemento adjacente, o binário é formado por forças de valores $\left(\mathbf{m}_{12}+\mathbf{m}_{12,2} \cdot \mathbf{d x} \mathbf{x}_{2}\right)$. A soma destas duas forças resulta em $\mathbf{m}_{12,2}$ e, adicionando a esta o esforço $q_{1}$, obtém-se a força cortante equivalente. Logo, pode-se escrever que:

$$
V_{i}=q_{i}+m_{i j, j} \cdot d x_{j} \quad(i \neq j=1,2)
$$

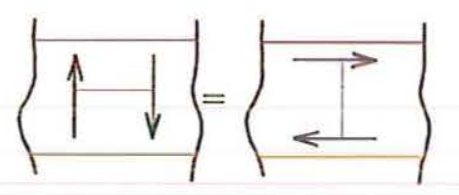

(a)
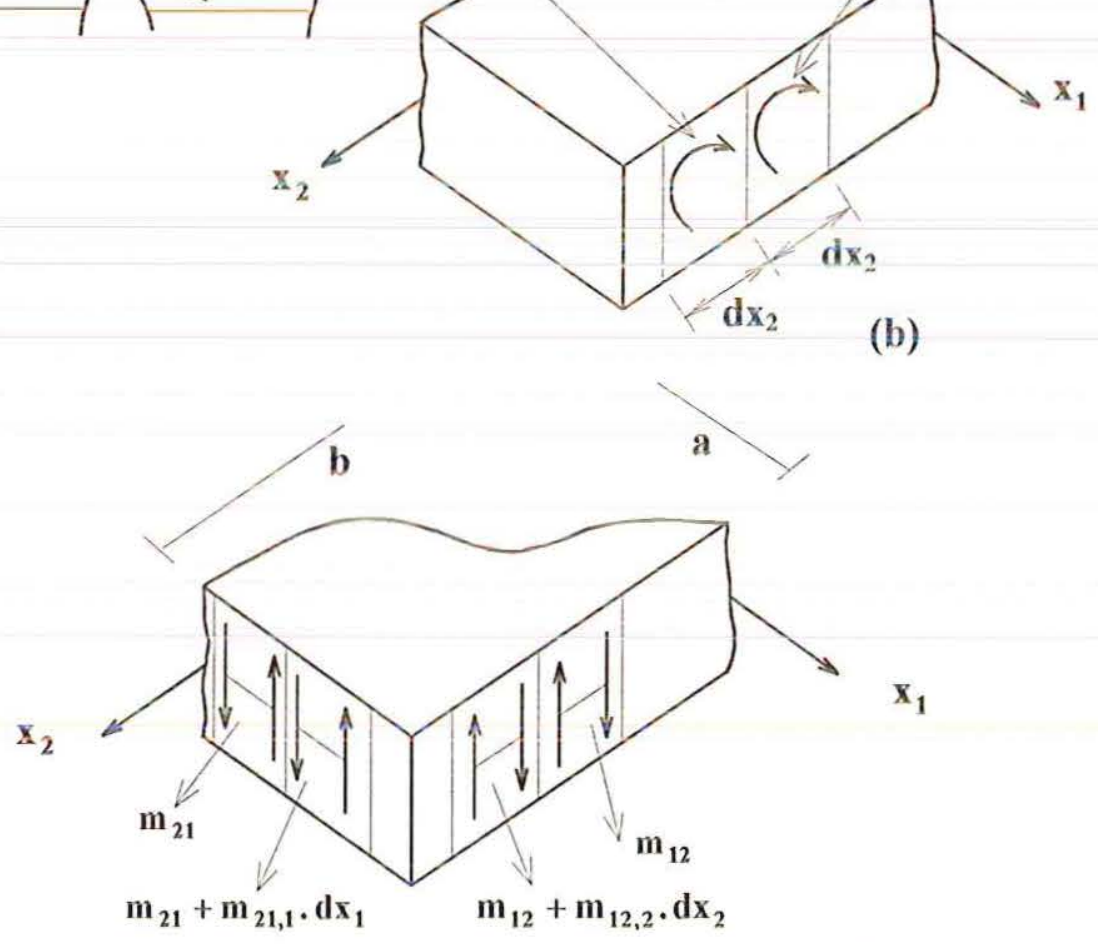

(c)

FIGURA 2.19 - Condições nas bordas. 
Um fenômeno interessante ocorre nos cantos da placa onde concentrações de forças são geradas devido ao momento volvente adjacente ao canto, figura 2.20. Isto significa que, quando uma placa é simplesmente apoiada, as forças de reação inclui, não apenas as forças cortantes equivalentes ao longo da borda, mas também, a reação de canto $\mathbf{R}_{\mathbf{c}}$. Se não há suporte no canto, este tende a levantar ou a descer dependendo do sinal de $\mathbf{m}_{12}$.

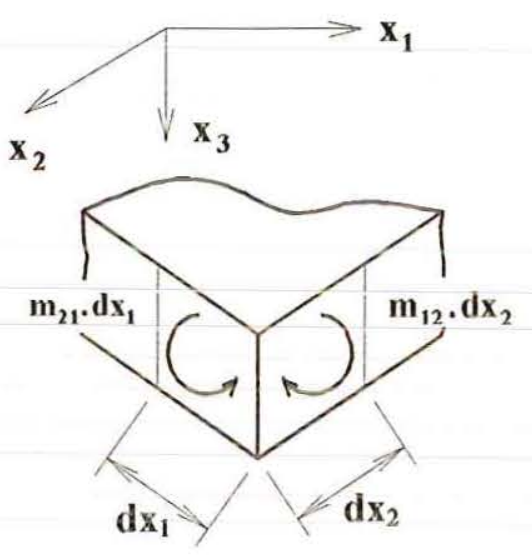

(a)

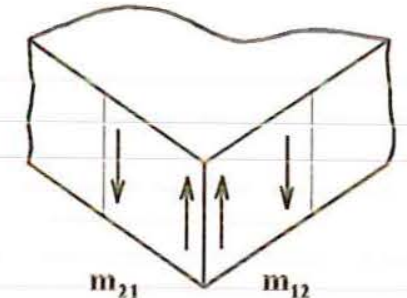

(b)

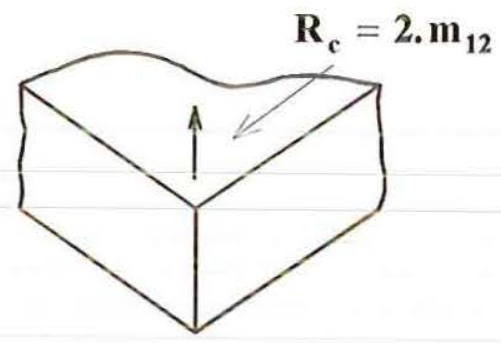

(c)

FIGURA 2.20 - Reação de Canto.

\subsection{Condiçóes de Contorno}

Serão abordados aqui as diferentes condições de restrições do contorno da placa, sendo as mais usuais:

1 - Borda engastada:

A borda tem os deslocamentos nulos:

$$
w=\theta_{\mathrm{X}_{1}}=\theta_{\mathrm{X}_{2}}=\mathbf{0}
$$

2 - Borda simplesmente apoiada

Admitem-se duas possibilidades: 


\section{1 - Condição forte:}

Os deslocamentos transversais e as rotações na direção da borda são nulos:

$$
w=\theta_{\mathrm{s}}=\mathbf{0}
$$

onde s é a direção da borda apoiada.

2.2 - Condição fraca:

São restritos apenas os deslocamentos transversais:

$$
w=0
$$

3 - Borda Livre

$$
\mathbf{M}_{\mathbf{i}}=\mathbf{0} \quad \mathrm{V}_{\mathbf{i}}=\mathbf{0}
$$

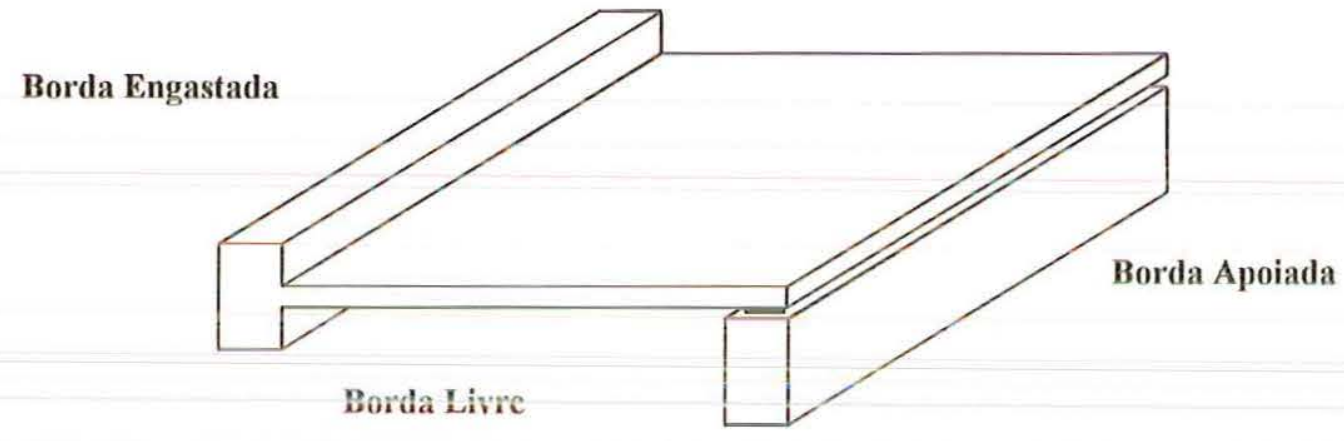

FIGURA 2.21 - Placa com Condições de Contorno.

\subsection{Placa com Espessura Variável}

Para problema de flexão de placas retangulares com espessura variável, assumindo-se que não haja variação brusca da espessura, as equações (2.20), (2.21) (2.22) são válidas. Escrevendo-se a equação diferencial da placa em função do 
momento como na equação (2.26) e observando que a rigidez à flexão da placa $\mathbf{D}\left(\mathbf{x}_{1}, \mathbf{x}_{2}\right)$ não é mais constante e sim uma função de $\mathbf{x}_{1}$ e $\mathbf{x}_{2}$, TIMOSHENKO \& KRIEGER (1959), obtém-se:

D. $\nabla^{2} \nabla^{2} w+2 \cdot \frac{\partial \mathrm{D}}{\partial \mathrm{x}_{1}} \cdot \frac{\partial}{\partial \mathrm{x}_{1}} \nabla^{2} w+2 \cdot \frac{\partial \mathrm{D}}{\partial \mathrm{x}_{2}} \cdot \frac{\partial}{\partial \mathrm{x}_{2}} \nabla^{2} w+\nabla^{2} \mathrm{D} \cdot \nabla^{2} w$

$$
-(1-v) \cdot\left(\frac{\partial^{2} \mathrm{D}}{\partial \mathrm{x}_{1}^{2}} \cdot \frac{\partial^{2} w}{\partial \mathbf{x}_{2}^{2}}-2 \cdot \frac{\partial^{2} \mathrm{D}}{\partial \mathrm{x}_{1} \cdot \partial \mathrm{x}_{2}} \cdot \frac{\partial^{2} w}{\partial \mathrm{x}_{1} \cdot \partial \mathrm{x}_{2}}+\frac{\partial^{2} \mathrm{D}}{\partial \mathbf{x}_{2}^{2}} \cdot \frac{\partial^{2} w}{\partial \mathbf{x}_{1}^{2}}\right)=\mathbf{g}
$$

onde :

$$
D\left(x_{1}, x_{2}\right)=\frac{E \cdot t^{3}\left(x_{1}, x_{2}\right)}{12 \cdot(1-v)}
$$

$\nabla^{2}$ é o operador de Laplace dado por:

$$
\nabla^{2}=\frac{\partial^{2}}{\partial \mathbf{x}_{1}^{2}}+\frac{\partial^{2}}{\partial \mathbf{x}_{2}^{2}}
$$

Neste trabalho será considerado variação linear da rigidez, com isso ter-se-á variação raiz cúbica da espessura, figura 2.22.

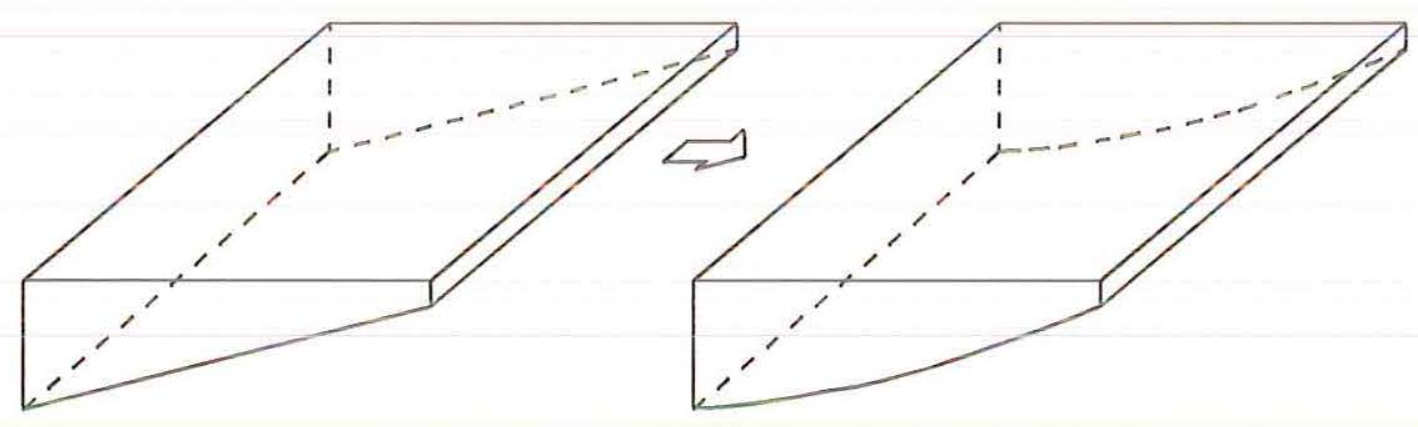

(a) Rigidez Linear

(b) Espessura - raiz cúbica

FIGURA 2.22 - Variação Linear da Rigidez. 


\subsection{Esforços Segundo um Sistema Genérico de Coordenadas}

Os momentos de flexão e volvente e as forças cortantes podem ser escritos também em relação a um outro sistema qualquer de coordenadas $(\mathbf{n}, \mathbf{s})$ ortogonais, como é mostrado na figura 2.23 .

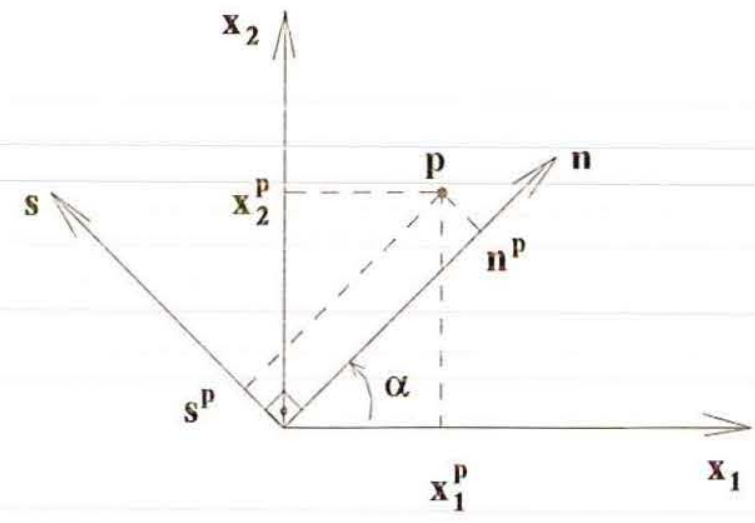

FIGURA 2.23 - Sistemas de Coordenadas $\left(\mathbf{x}_{1}, \mathbf{x}_{2}\right)$ e $(\mathbf{n}, \mathbf{s})$

A transformação entre os sistemas de coordenadas $(x, y)$ e $(\mathbf{n}, \mathbf{s})$ pode ser escrita na forma matricial como:

$$
\begin{gathered}
\left\{\begin{array}{l}
x_{1}^{p} \\
x_{2}^{p}
\end{array}\right\}=[T] \cdot\left\{\begin{array}{l}
n^{p} \\
s^{p}
\end{array}\right\} \\
\left\{\begin{array}{l}
n^{p} \\
s^{p}
\end{array}\right\}=[T]^{T} \cdot\left\{\begin{array}{l}
x_{1}^{p} \\
x_{2}^{p}
\end{array}\right\}
\end{gathered}
$$

onde a matriz de transformação [T] é dada por: 


$$
[T]=\left[\begin{array}{ll}
n_{1} & s_{1} \\
n_{2} & s_{2}
\end{array}\right]=\left[\begin{array}{ll}
\cos (\alpha) & -\operatorname{sen}(\alpha) \\
\operatorname{sen}(\alpha) & \cos (\alpha)
\end{array}\right]
$$

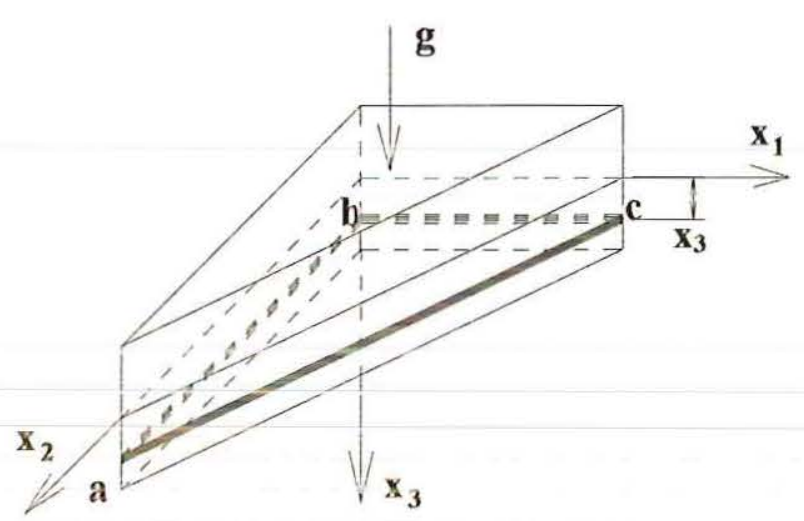

(a)

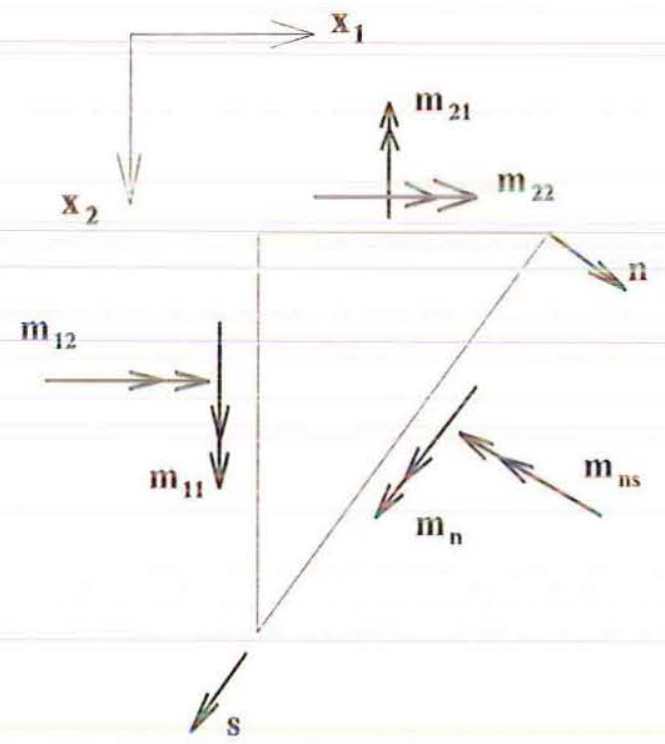

(c)

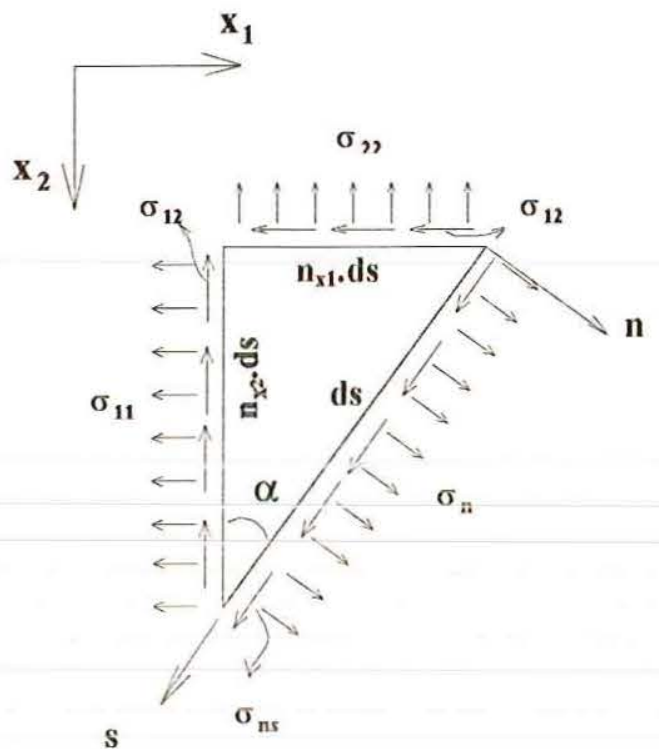

(b)

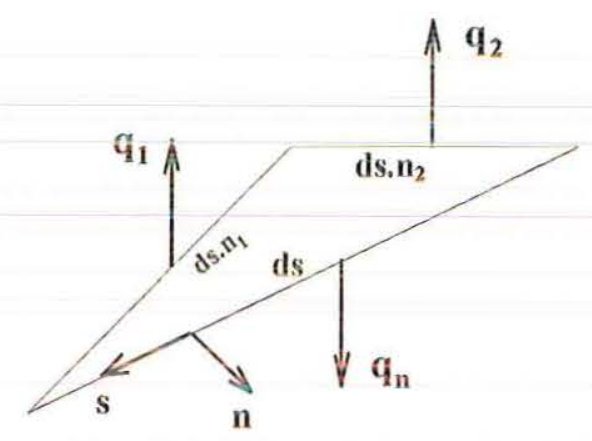

(d)

FIGURA 2.24 - Tensões e Esforços no Elemento "abc". 
Considere-se o elemento de placa "abc" da figura 2.24a, cujo plano é paralelo ao plano médio e distanciado de $\mathbf{x}_{\mathbf{3}}$ deste plano. Fazendo-se o equilíbrio do elemento mostrado na figura 2.24b e utilizando-se convenientemente as expressões (2.32), obtêm-se as tensões normais e tangenciais no sistema $(\mathbf{n}, \mathbf{s})$ a partir das tensões conhecidas no sistema $\left(\mathbf{x}_{1}, \mathbf{x}_{2}\right)$. Logo:

$$
\left\{\begin{array}{l}
\sigma_{n}=\sigma_{i j} \cdot n_{i} \cdot n_{j} \\
\sigma_{n s}=\sigma_{i j} \cdot n_{i} \cdot s_{j} \\
\sigma_{s}=\sigma_{i j} \cdot s_{i} \cdot s_{j}
\end{array} \quad(i, j=1,2)\right.
$$

Os momentos atuantes na face ac do elemento $\left(\mathbf{M}_{\mathbf{n}}, \mathbf{M}_{\mathbf{s}}\right.$ e $\left.\mathbf{M}_{\mathbf{n s}}\right)$, figura $2.24 c$, são obtidos por:

$$
M_{n}=\int_{-t / 2}^{+t / 2} x_{3} \cdot \sigma_{n} \cdot d x_{3}, \quad M_{s}=\int_{-t / 2}^{+t / 2} x_{3} \cdot \sigma_{s} \cdot d x_{3}, \quad M_{n s}=\int_{-t / 2}^{+t / 2} x_{3} \cdot \sigma_{n s} \cdot d x_{3}
$$

Utilizando-se das equações (2.34) e considerando as equações (2.16), obtémse:

$$
\begin{aligned}
& M_{n}=m_{i j} \cdot n_{i} \cdot n_{j} \\
& M_{s}=m_{i j} \cdot s_{i} \cdot s_{j} \\
& M_{n s}=m_{i j} \cdot n_{i j} \cdot s_{j}
\end{aligned}
$$

Da mesma forma que as tensões, a força cortante atuante na face ac pode ser relacionada com as forças cortantes relativas aos eixos $x_{1}$ e $x_{2}$, através do equilíbrio das forças verticais, figura $2.24 \mathrm{~d}$, resultando em:

$$
\mathbf{q}_{\mathbf{n}}=\mathbf{q}_{\mathbf{i}} \cdot \mathbf{n}_{\mathbf{i}}
$$

e com isso, a força cortante equivalente deste sistema fica:

$$
\mathbf{V}_{\mathbf{n}}=\mathbf{q}_{\mathbf{n}}+\frac{\partial \mathbf{M}_{\mathbf{n s}}}{\partial \mathbf{s}}
$$



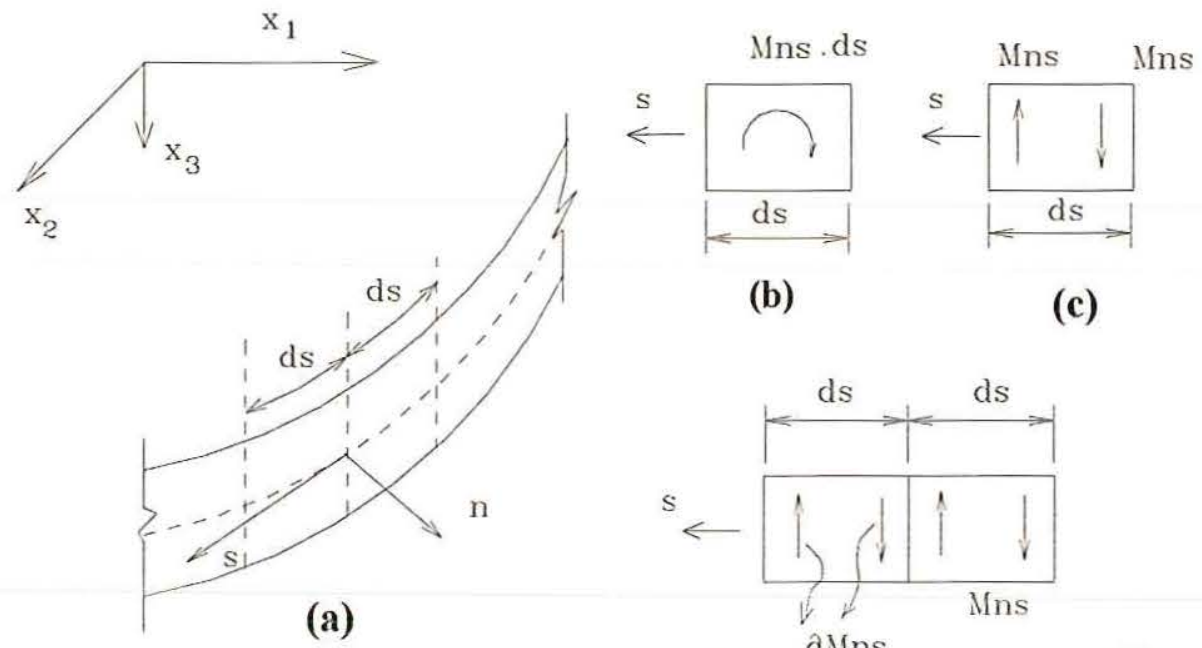

(b)

(c)

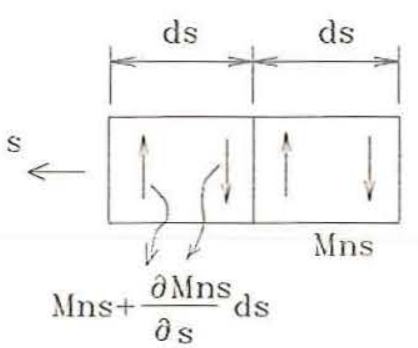

(d)

FIGURA 2.25 - Momento Volvente na Borda.

\subsection{Equações de Placas em Coordenadas Polares}

Na solução de certos problemas é conveniente que a equação diferencial, assim como as outras relações, estejam referidas a um sistema de coordenadas polares. É o caso da solução fundamental para problemas de placas.

Para isso, considere-se os sistemas de coordenadas cartesianas e polares definidas sobre o plano, figura 2.26. As coordenadas $\left(x_{1}^{p}, x_{2}^{p}\right)$ de um ponto podem ser definidas em função de r e $\theta$.

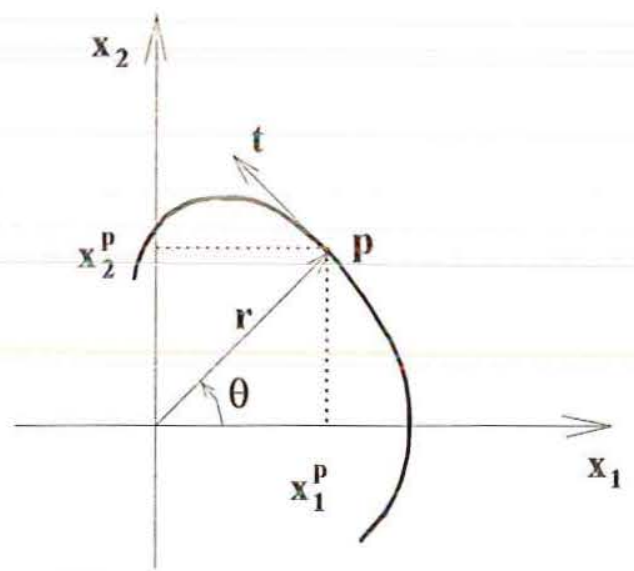

FIGURA 2.26 - Sistema de Coordenadas Cartesianas e Polares. 
Da figura 2.26, pode-se tirar as seguintes relações:

$$
\begin{aligned}
& x_{1}=r_{1}=r \cdot \cos (\theta) \\
& x_{2}=r_{2}=r \cdot \operatorname{sen}(\theta) \\
& r^{2}=x_{1}^{2}+x_{2}^{2}=r_{i} \cdot r_{i} \\
& \operatorname{tg}(\theta)=\frac{x_{2}}{x_{1}} \quad \Rightarrow \theta=\operatorname{arctg}\left(\frac{x_{2}}{x_{1}}\right)
\end{aligned}
$$

Derivando-se (2.39.c) em função de $\mathbf{x}_{1}$ e de $\mathbf{x}_{2}$, obtém-se respectivamente:

$$
r_{1}=\frac{r_{1}}{r}=\cos (\theta)
$$

$$
r_{2}=\frac{r_{2}}{r}=\operatorname{sen}(\theta)
$$

Derivando-se (2.39.d) em função de $x_{1}$ e de $x_{2}$, obtém-se:

$$
\begin{aligned}
& \theta,_{1}=-\frac{x_{2}}{r^{2}}=-\frac{r_{2}}{r^{2}}=-\frac{\operatorname{sen}(\theta)}{r} \\
& \theta,{ }_{2}=\frac{x_{1}}{r^{2}}=\frac{r_{1}}{r^{2}}=\frac{\cos (\theta)}{r}
\end{aligned}
$$

Considerando-se a função dos deslocamentos transversais w como uma função de $\mathbf{r}$ e $\theta$, a derivada de $w(\mathbf{r}, \theta)$ em relação a $\mathbf{x}_{\mathbf{i}}$ fica:

$$
w_{\mathbf{i}_{\mathbf{i}}}=\frac{\partial w}{\partial \mathbf{r}} \cdot \mathbf{r}_{\mathbf{i}}+\frac{\partial w}{\partial \theta} \cdot \theta,_{\mathbf{i}} \quad(\mathbf{i}=1,2)
$$


A partir de (2.41), pode-se definir o operador diferencial de primeira ordem, dado por:

$$
\frac{\partial}{\partial \mathbf{x}_{\mathbf{i}}}=\mathbf{r},{ }_{\mathbf{i}} \cdot \frac{\partial}{\partial \mathbf{r}}+\theta_{\mathbf{i}_{\mathbf{i}}} \cdot \frac{\partial}{\partial \theta}
$$

Com isso, a segunda derivada pode ser expressa por:

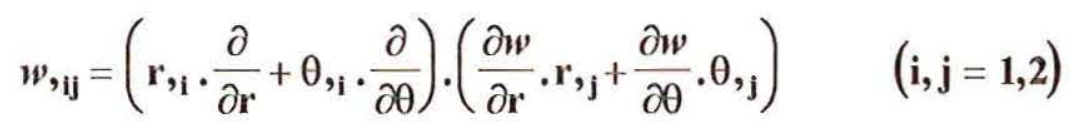

Desenvolvendo-se as derivadas, obtém-se:

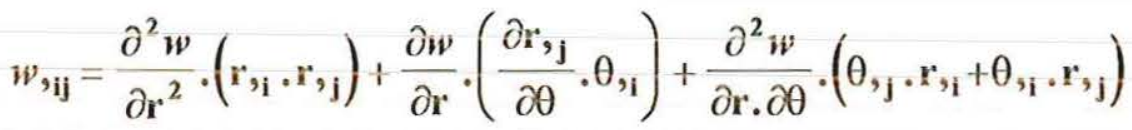

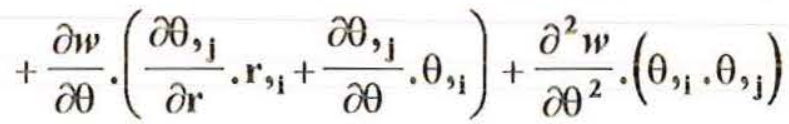

onde:

$$
\begin{aligned}
& \frac{\partial \mathbf{r}, 1}{\partial \theta}=-\operatorname{sen}(\theta) \\
& \frac{\partial \mathbf{r},_{2}}{\partial \theta}=\cos (\theta) \\
& \frac{\partial \theta_{1}}{\partial r}=\frac{\operatorname{sen}(\theta)}{\mathbf{r}^{2}} \\
& \frac{\partial \theta_{2}}{\partial r}=-\frac{\cos (\theta)}{r^{2}}
\end{aligned}
$$

Somando-se as expressões (2.44) para $\mathbf{i}=\mathbf{j}=\mathbf{1}$ e $\mathbf{i}=\mathbf{j}=\mathbf{2}$ e fazendo-se as devidas simplificações, obtém-se:

$$
\nabla^{2} w=\left(\frac{\partial^{2}}{\partial \mathbf{x}_{1}^{2}}+\frac{\partial^{2}}{\partial \mathbf{x}_{2}^{2}}\right) \cdot w=\left(\frac{\partial^{2}}{\partial \mathbf{r}^{2}}+\frac{1}{\mathbf{r}} \cdot \frac{\partial}{\partial \mathbf{r}}+\frac{1}{\mathbf{r}^{2}} \cdot \frac{\partial^{2}}{\partial \theta^{2}}\right) \cdot w
$$


Portanto, a equação diferencial de placas, em coordenadas polares, fica dada por:

$$
\nabla^{2} \nabla^{2} w=\left(\frac{\partial^{2}}{\partial \mathbf{r}^{2}}+\frac{1}{\mathbf{r}} \cdot \frac{\partial}{\partial \mathbf{r}}+\frac{1}{\mathbf{r}^{2}} \cdot \frac{\partial^{2}}{\partial \theta^{2}}\right) \cdot\left(\frac{\partial^{2} w}{\partial \mathbf{r}^{2}}+\frac{1}{\mathrm{r}} \cdot \frac{\partial w}{\partial \mathbf{r}}+\frac{1}{\mathbf{r}^{2}} \cdot \frac{\partial^{2} w}{\partial \theta^{2}}\right)=\frac{\mathrm{g}}{\mathrm{D}}
$$

Substituindo-se a eq.(2.43) na eq.(2.18) e na eq.(2.24), obtêm-se os momentos fletores e forças cortantes em coordenadas polares:

$$
\mathbf{m}_{\mathbf{i j}}=-\mathbf{D} \cdot\left\{\frac{\mathbf{d}^{2} w}{\mathbf{d r}^{2}} \cdot\left[\delta_{\mathrm{ij}} \cdot v+(1-v) \cdot\left(\mathbf{r},{ }_{\mathrm{i}} \cdot \mathbf{r}, \mathbf{j}\right)\right]+\frac{1}{\mathbf{r}} \cdot \frac{\mathbf{d} w}{\mathbf{d r}} \cdot\left[\delta_{\mathrm{ij}} \cdot v+(1-v) \cdot\left(\mathbf{t}_{\mathbf{i}} \cdot \mathbf{t}_{\mathbf{j}}\right)\right]\right\}
$$

$$
\mathbf{q}_{\mathrm{i}}=-\mathbf{D} \cdot \frac{\partial}{\partial \mathbf{x}_{\mathbf{i}}} \cdot \nabla^{2} w=-\mathbf{D} \cdot\left[\mathbf{r}, \frac{\partial}{\partial \mathbf{r}} \nabla^{2} w-\theta, \frac{\partial}{\partial \theta} \nabla^{2} w\right]
$$

onde $\boldsymbol{t}_{\mathbf{i}}$ são os co-senos diretores do versor $\mathbf{t}$, indicado na figura 2.26 , dados por:

$$
\begin{array}{r}
t_{1}=-r,_{2}=-\operatorname{sen}(\theta) \\
t_{2}=r_{,_{1}}=\cos (\theta)
\end{array}
$$

Para todo problema que tem simetria em relação à origem do sistema de coordenadas polares, como ocorre no problema fundamental de placas, pode ser mostrado que o deslocamento $w$ não é função de $\theta$. O deslocamento $w$ é função apenas de $\mathbf{r}$ e a equação diferencial de placas em coordenadas polares (2.47), se reduz $\mathrm{a}$ :

$$
\nabla^{2} \nabla^{2} w=\left(\frac{\mathrm{d}^{2}}{\mathrm{dr}^{2}}+\frac{1}{\mathrm{r}} \cdot \frac{\mathrm{d}}{\mathrm{dr}}\right) \cdot\left(\frac{\mathrm{d}^{2} w}{\mathrm{dr}^{2}}+\frac{1}{\mathrm{r}} \cdot \frac{\mathrm{d} w}{\mathrm{dr}}\right)=\frac{\mathrm{g}}{\mathrm{D}}
$$

ou

$$
\frac{d^{4} w}{d r^{4}}+\frac{2}{r} \cdot \frac{d^{3} w}{d r^{3}}-\frac{1}{r^{2}} \cdot \frac{d^{2} w}{d r^{2}}+\frac{1}{r^{3}} \cdot \frac{d w}{d r}=\frac{g}{D}
$$


Utilizando-se do operador:

$$
\nabla^{2}=\left(\frac{d^{2}}{d r^{2}}+\frac{1}{r} \cdot \frac{d}{d r}\right)
$$

obtém-se:

$$
\frac{d}{d r} \cdot\left(\frac{d^{2}}{d r^{2}}+\frac{1}{r} \cdot \frac{d}{d r}\right)=\left(\frac{d^{3}}{d r^{3}}+\frac{1}{r} \cdot \frac{d^{2}}{d r^{2}}-\frac{1}{r^{2}} \cdot \frac{d}{d r}\right)
$$

que possibilita obter:

$$
w_{9_{\mathrm{kki}}}=\mathrm{r}_{9_{\mathrm{i}}} \cdot\left(\frac{\mathrm{d}^{3} w}{\mathrm{dr} \mathbf{r}^{3}}+\frac{1}{\mathrm{r}} \cdot \frac{\mathrm{d}^{2} w}{\mathrm{dr}^{2}}-\frac{1}{\mathrm{r}^{2}} \cdot \frac{\mathrm{d} w}{\mathrm{dr}}\right) \quad(\mathrm{i}, \mathrm{k}=1,2)
$$

Logo, o esforço cortante é dado por:

$$
\mathrm{q}_{\mathrm{i}}=-D \cdot \mathrm{r},\left(\frac{\mathrm{d}^{3} w}{\mathrm{dr} \mathrm{r}^{3}}+\frac{1}{\mathrm{r}} \cdot \frac{\mathrm{d}^{2} w}{\mathrm{dr}^{2}}-\frac{1}{\mathrm{r}^{2}} \cdot \frac{\mathrm{d} w}{\mathrm{dr}}\right) \quad(\mathrm{i}=1,2)
$$

Obtidas as expressões dos momentos e da força cortante em coordenadas polares, é interessante expressar estes valores em função das coordenadas $(\mathbf{n}, \mathbf{s}), \mathbf{M}_{\mathbf{n}}$, $\mathbf{M}_{\mathrm{ns}}$ e $\mathbf{V}_{\mathrm{n}}$, como definidas no item 2.11.

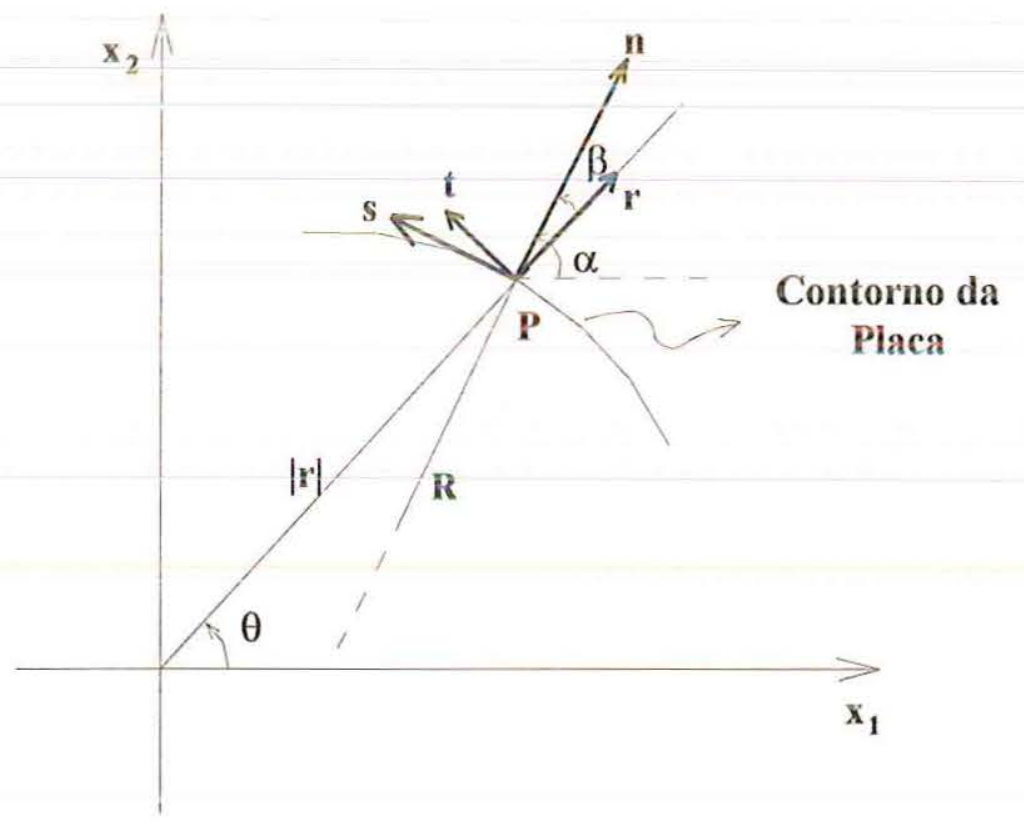

FIGURA 2.27 - Vetores n e s no Ponto P do Contorno da Placa. 
Substituindo-se a eq.(2.48) na eq.(2.36) e (2.56) em (2.37), obtêm-se as equações:

$$
\begin{aligned}
& M_{n}=-D \cdot\left\{\frac{d^{2} w}{d r^{2}} \cdot\left[v+(1-v) \cdot\left(r, r_{i} \cdot n_{i}\right)^{2}\right]+\frac{1}{r} \cdot \frac{d w}{d r} \cdot\left[v+(1-v) \cdot\left(r, r_{i} \cdot s_{i}\right)^{2}\right]\right\} \\
& M_{n s}=-D \cdot(1-v) \cdot\left(r_{i}, n_{i}\right) \cdot\left(r,,_{j} \cdot s_{j}\right) \cdot\left(\frac{d^{2} w}{d r^{2}}-\frac{1}{r} \cdot \frac{d w}{d r}\right) \\
& q_{n}=-D \cdot\left(r, n_{i} \cdot n_{i}\right) \cdot\left(\frac{d^{3} w}{d r^{3}}+\frac{1}{r} \cdot \frac{d^{2} w}{d r^{2}}-\frac{1}{r^{2}} \cdot \frac{d w}{d r}\right)
\end{aligned}
$$

Sendo:

$$
\begin{aligned}
& \mathbf{r}_{\mathbf{r}_{\mathbf{i}} \cdot \mathbf{n}_{\mathbf{i}}}=\cos (\beta) \\
& \mathbf{r}_{\mathbf{j}} \cdot \mathbf{s}_{\mathbf{j}}=\cos \left(\frac{\pi}{2}+\beta\right)=-\operatorname{sen}(\beta)
\end{aligned}
$$

a eq.(2.58) fica:

$$
M_{n s}=D \cdot(1-v) \cdot \cos (\beta) \cdot \operatorname{sen}(\beta) \cdot\left(\frac{d^{2} w}{d r^{2}}-\frac{1}{r} \cdot \frac{d w}{d r}\right)
$$

Derivando-se a eq.(2.58), que é função de $\mathrm{r}$ e $\beta$, em relação à coordenada $\mathrm{s}$ do contorno, obtém-se:

$$
\frac{\partial \mathbf{M}_{n s}}{\partial s}=\frac{\partial \mathbb{M}_{n s}}{\partial r} \cdot \frac{\partial r}{\partial s}+\frac{\partial M_{n s}}{\partial \beta} \cdot \frac{\partial \beta}{\partial s}
$$

As derivadas em relação a s podem ser escritas como:

$$
\begin{aligned}
& \frac{\partial \mathbf{r}}{\partial s}=\mathbf{r}_{\mathbf{i}_{\mathbf{i}}}, \mathbf{s}_{\mathbf{i}_{\mathbf{i}}}=-\operatorname{sen}(\beta) \\
& \frac{\partial \beta}{\partial \mathbf{s}}=\frac{1}{\mathbf{R}}-\frac{\cos (\beta)}{\mathbf{r}}
\end{aligned}
$$

onde $\mathbf{R}$ é o raio da curvatura do contorno no ponto $\mathbf{P}$, mostrado na figura 2.27 . 
Da eq. (2.61), obtêm-se as derivadas de $\mathbf{M}_{\mathbf{n s}}$ em relação a $\mathbf{r}$ e $\beta$, as quais, substituídas na eq.(2.62), juntamente com as equações (2.63), produzem a equação:

$$
\begin{aligned}
& \frac{\partial M_{n s}}{\partial s}=D \cdot(1-v) \cdot \cos (\beta) \cdot\left[\operatorname{sen}^{2}(\beta) \cdot\left(-\frac{d^{3} w}{d r^{3}}-\frac{1}{r} \cdot \frac{d^{2} w}{d r^{2}}+\frac{1}{r^{2}} \cdot \frac{d w}{d r}\right)\right. \\
& \left.+\frac{4 \cdot \operatorname{sen}^{2}(\beta)-1}{r} \cdot\left(\frac{d^{2} w}{d r^{2}}-\frac{1}{r} \cdot \frac{d w}{d r}\right)\right]+D \cdot \frac{(1-v)}{R} \cdot\left(1-2 \cdot \operatorname{sen}^{2}(\beta)\right) \cdot\left(\frac{d^{2} w}{d r^{2}}-\frac{1}{r} \cdot \frac{d w}{d r}\right)
\end{aligned}
$$

Uma vez conhecidas as expressões de $\boldsymbol{q}_{\mathbf{n}}$ e $\frac{\partial \mathbf{M}_{\mathbf{n s}}}{\partial \mathbf{s}}$, dadas em (2.59) e (2.64), respectivamente, obtém-se a força cortante equivalente $\mathbf{V}_{\mathbf{n}}$ em coordenadas polares:

$$
\begin{aligned}
V_{n}= & -D \cdot(1-v) \cdot\left(n_{i} \cdot r_{i_{i}}\right) \cdot\left\{\left[\left(s_{j} \cdot r_{j}\right)^{2}+\frac{1}{1-v}\right] \cdot\left(\frac{d^{3} w}{d r^{3}}+\frac{1}{r} \cdot \frac{d^{2} w}{d r^{2}}-\frac{1}{r^{2}} \cdot \frac{d w}{d r}\right)\right. \\
& \left.+\frac{1-4 \cdot\left(s_{j} \cdot r_{g_{j}}\right)^{2}}{r} \cdot\left(\frac{d^{2} w}{d r^{2}}-\frac{1}{r} \cdot \frac{d w}{d r}\right)\right\}+\frac{D(1-v)}{r} \cdot\left[1-2 \cdot\left(s_{i} \cdot r,_{i}\right)^{2}\right] \cdot\left(\frac{d^{2} w}{d r^{2}}-\frac{1}{r} \cdot \frac{d w}{d r}\right)
\end{aligned}
$$

Além dos esforços de contorno, é necessário obter a expressão de $\frac{\partial w}{\partial \mathbf{n}}$ em coordenadas polares. Lembrando-se que, para casos que apresentam simetria, $w$ é função apenas de $\mathrm{r}$ e sua derivada direcional é dada por:

$$
\frac{\partial w}{\partial \mathbf{n}}=\frac{\mathbf{d} w}{\mathbf{d r}} \cdot \frac{\partial \mathbf{r}}{\partial \mathbf{n}}
$$

Como $\frac{\partial \mathbf{r}}{\partial \mathbf{n}}=\left(\mathbf{n}_{\mathbf{i}} \cdot \mathbf{r}, \mathbf{i}_{\mathbf{i}}\right)$, a eq.(2.66) fica:

$$
\frac{\partial w}{\partial \mathbf{n}}=\left(\mathbf{n}_{\mathbf{i}} \cdot \mathbf{r}, \mathbf{i}\right) \cdot \frac{\mathbf{d} w}{\mathbf{d r}}
$$




\subsection{Soluções Fundamentais de Placas}

Para problemas que têm como base o uso de equações integrais, é necessário o estudo das soluções fundamentais. Entende-se por solução fundamental a resposta em um ponto genérico "p" (Ponto Campo) de um domínio infinito, devido à aplicação de uma carga unitária em outro ponto " $q$ " (Ponto Fonte) deste mesmo domínio, figura 2.28 .

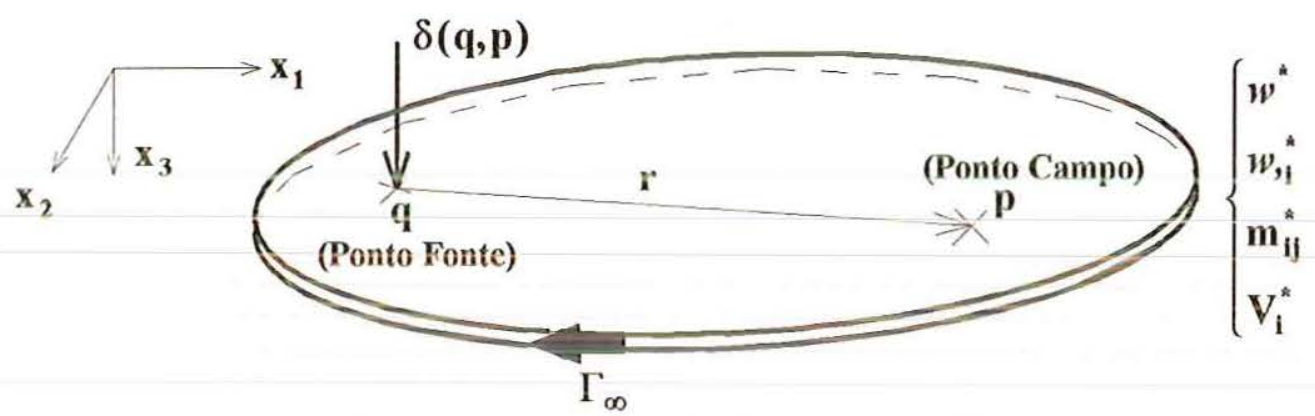

FIGURA 2.28 - Resposta em “p” Devido a Distribuição Delta de Dirac $\delta(q, p)$.

Pode-se definir este carregamento unitário através da distribuição delta de Dirac, denotada por $\delta(q, p)$, cujas propriedades são:

$$
\left\{\begin{array}{cc}
\delta(\mathbf{q}, \mathbf{p})=0, & \text { para } \mathbf{p} \neq \mathbf{q} \\
\delta(\mathbf{q}, \mathbf{p})=\infty, & \text { para } \mathbf{p} \equiv \mathbf{q}
\end{array}\right.
$$

Para uma função contínua qualquer $\phi$, tem-se que:

$$
\int_{\Omega_{\infty}} \phi(p) \cdot \delta(q, p) \cdot d \Omega_{\infty}=\phi(q)
$$

uma vez que: 


$$
\int_{\Omega_{\infty}} \delta(q, p) \cdot d \Omega_{\infty}=1
$$

Assim, conclui-se que a resultante do carregamento definido pela distribuição delta de Dirac sobre o domínio fundamental é uma força unitária aplicada no ponto "q".

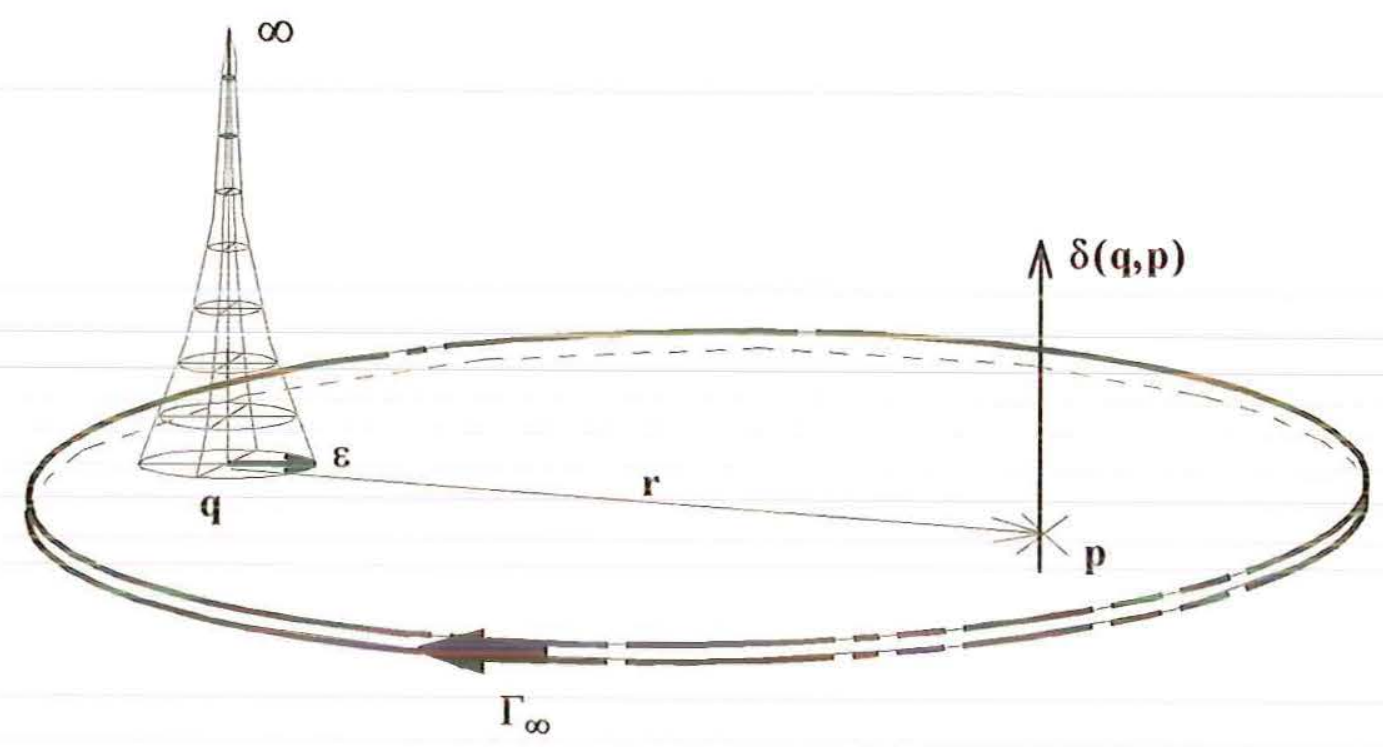

FIGURA 2.29 - Função Delta de Dirac.

Portanto, a solução fundamental é obtida a partir da equação diferencial (2.51), substituindo-se g pela distribuição delta de Dirac, isto é:

$$
\nabla^{2} \nabla^{2} w^{*}=\frac{\delta(q, p)}{D}
$$

onde $w^{*}$ representa a solução fundamental que é obtida pela resolução da equação diferencial (2.71), para todos os pontos do domínio fundamental, com exceção do ponto de carregamento " $q$ ". Nestas condições, a equação diferencial fica:

$$
\nabla^{2} \nabla^{2} w^{*}=0
$$


Considerando-se um sistema de coordenadas polares com origem em q e levando-se em conta a simetria existente e também a eq.(2.51), que considera $w$ apenas como função de $\mathbf{r}$, já que não varia $\operatorname{com} \theta$, pode-se escrever:

$$
\nabla^{2} \nabla^{2} w^{*}=\left(\frac{\mathrm{d}^{2}}{\mathrm{dr} \mathbf{r}^{2}}+\frac{1}{\mathrm{r}} \cdot \frac{\mathrm{d}}{\mathrm{dr}}\right) \cdot\left(\frac{\mathrm{d}^{2} w^{*}}{\mathrm{dr}^{2}}+\frac{1}{\mathrm{r}} \cdot \frac{\mathrm{d} w^{*}}{\mathrm{dr}}\right)=0
$$

ou seja,

$$
\frac{d^{4} w^{*}}{d r^{4}}+\frac{2}{r} \cdot \frac{d^{3} w^{*}}{d r^{3}}-\frac{1}{r^{2}} \cdot \frac{d^{2} w^{*}}{d r^{2}}+\frac{1}{r^{3}} \cdot \frac{d w^{*}}{d r}=0
$$

A equação diferencial homogênea (2.73) pode ser rescrita, utilizando a identidade:

$$
\frac{\mathrm{d}^{2} w^{*}}{\mathrm{dr} \mathbf{r}^{2}}+\frac{1}{\mathrm{r}} \cdot \frac{\mathrm{d} w^{*}}{\mathrm{dr}}=\frac{1}{\mathrm{r}} \cdot \frac{\mathrm{d}}{\mathrm{dr}}\left(\mathrm{r} \cdot \frac{\mathrm{d} w^{*}}{\mathrm{dr}}\right)
$$

resultando:

$$
\frac{1}{r} \frac{d}{d r}\left\{r \cdot \frac{d}{d r}\left[\frac{1}{r} \frac{d}{d r}\left(r \cdot \frac{d \omega^{*}}{d r}\right)\right]\right\}=0
$$

Fazendo-se integrações sucessivas, obtém-se o deslocamento $w^{\star 2}$ :

$$
w^{*}=\frac{C_{1}}{4} \cdot r^{2} \cdot \ln (r)+\left(C_{2}-C_{1}\right) \cdot \frac{r^{2}}{8}+C_{3} \cdot \ln (r)+C_{4}
$$

Levando-se em consideração a condição de simetria em relação ao ponto " $q$ ", figura 2.30 , pode-se escrever que $\frac{\mathbf{d} \boldsymbol{w}^{*}}{\mathbf{d r}}=\mathbf{0}$, para $\mathrm{r}=\mathbf{0}$, obtendo-se, da eq. (2.77):

$$
w^{*}=\mathrm{C}_{4}+\mathrm{C}_{3} \cdot \ln (\mathrm{r}=0)
$$

A expressão (2.78) indica que o deslocamento é infinito. Para que isto não ocorra, a seguinte condição deve ser satisfeita:

$$
\mathrm{C}_{3}=\mathbf{0}
$$


A constante $\mathbf{C}_{1}$ pode ser obtida a partir da condição de equilíbrio das forças verticais atuantes em um círculo de raio $r$, cujo centro é o ponto " $q$ " de aplicação da carga unitária, como mostra a figura 2.30 .
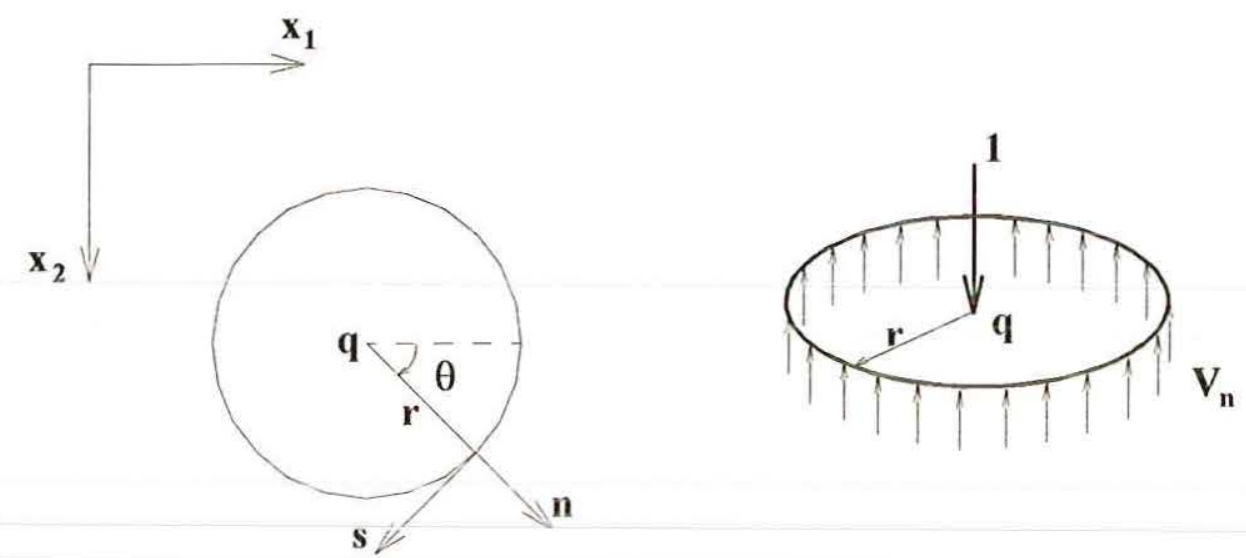

FIGURA 2.30 - Forças Atuantes no Círculo de Raio r e Centro q.

Assim, a força cortante equivalente $\mathbf{V}_{\mathbf{n}}$ em um ponto " $\mathbf{p}$ " da circunferência, necessária para equilibrar a carga unitária, vale:

$$
V_{n}=-\frac{1}{2 \cdot \pi \cdot r}
$$

A expressão de $V_{n}$, dada na eq..(2.65), neste caso, é apenas função de $r$, já que o ângulo $\beta$ é nulo para todos os pontos da circunferência. Portanto, tem-se:

$$
V_{n}=-D \cdot\left(\frac{d^{3} w^{*}}{d r^{3}}+\frac{1}{r} \cdot \frac{d^{2} w^{*}}{d r^{2}}-\frac{1}{r^{2}} \cdot \frac{d w^{*}}{d r}\right)
$$

Substituindo-se (2.81) em (2.80) e expressando-se $V_{n}$ em função do Laplaciano em coordenadas polares, tem-se:

$$
-\mathrm{D} \cdot \frac{\mathrm{d}}{\mathrm{dr}}\left(\frac{\mathrm{d}^{2} w^{4}}{\mathrm{dr} \mathrm{r}^{2}}+\frac{1}{\mathrm{r}} \cdot \frac{\mathrm{d} w^{4}}{\mathrm{dr}}\right)=-\frac{1}{2 \cdot \pi \cdot \mathrm{r}}
$$


Das equações (2.77) e (2.82), obtém-se:

$$
C_{1}=\frac{1}{2 . \pi \cdot D}
$$

Este valor, substituído em (2.77), juntamente com (2.79), leva a:

$$
w^{*}=\frac{1}{8 \cdot \pi \cdot \mathrm{D}} \cdot \mathrm{r}^{2} \cdot \ln (\mathrm{r})+\left(\mathrm{C}_{2}-\frac{1}{2 \cdot \pi \cdot \mathrm{D}}\right) \cdot \frac{\mathrm{r}^{2}}{8}+\mathrm{C}_{4}
$$

As constantes $\mathbf{C}_{2}$ e $\mathbf{C}_{4}$, no caso da placa fundamental, podem assumir quaisquer valores. STERN (1979) e BEZINE (1978) apud PAIVA (1987) adotam valores nulos. DANSON (1979) apud PAIVA (1987), adota os seguintes:

$$
\begin{gathered}
C_{2}=-\frac{1}{2 . \pi \cdot D} \\
C_{4}=0
\end{gathered}
$$

Substituindo-se (2.85) e (2.86) na solução fundamental $w^{*}$ dada pela eq. (2.84), resulta:

$$
w^{*}=\frac{1}{8 \cdot \pi \cdot \mathrm{D}} \cdot \mathrm{r}^{2} \cdot\left(\ln (\mathrm{r})-\frac{1}{2}\right)
$$

onde:

$$
r=\left\{[x(p)-x(q)]^{2}+[y(p)-y(q)]^{2}\right\}^{1 / 2}
$$

Conforme se verá no próximo capítulo, é necessário determinar a solução fundamental da derivada do deslocamento e dos esforços definidos no ponto "p", em relação ao sistema (n,s), figura 2.31 . 


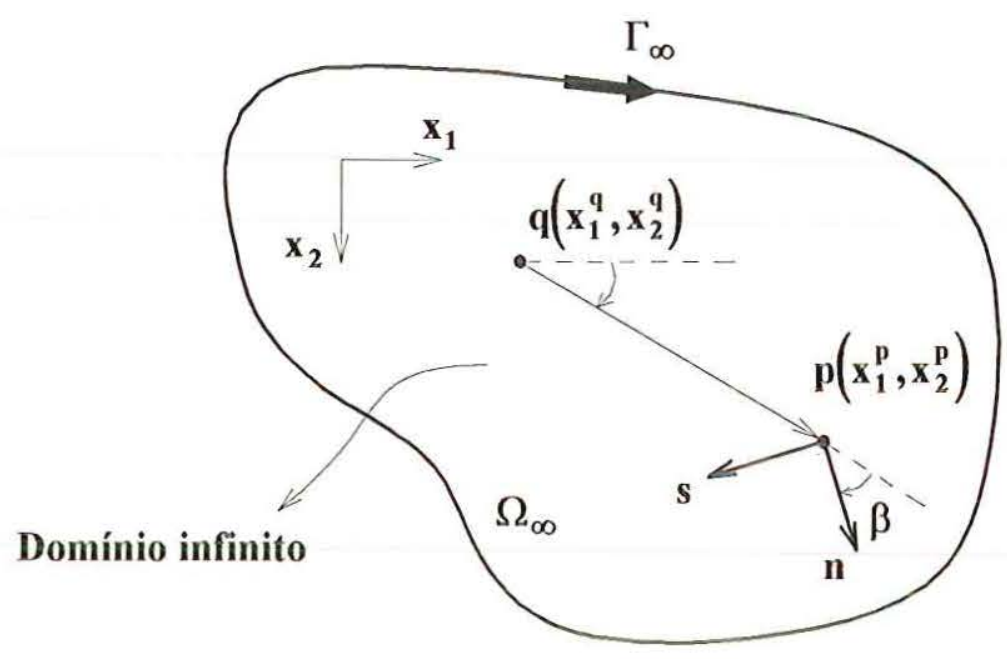

FIGURA 2.31 - Pontos de Carregamento "q" e de Resposta " $p$ ".

Assim a eq.(2.66), para solução fundamental, fica:

$$
\frac{\partial w^{*}}{\partial \mathbf{n}}=\frac{\mathbf{d} w^{*}}{\mathbf{d r}} \cdot \frac{\partial \mathbf{r}}{\partial \mathrm{n}}
$$

Como a derivada está sendo calculada no ponto "p", tem-se:

$$
\frac{\partial r}{\partial n}=\frac{\partial r}{\partial x_{i}(p)} \cdot \frac{\partial x_{i}(p)}{\partial n}=r_{i} \cdot n_{i}
$$

A partir da eq.(2.88), obtém-se:

$$
\begin{gathered}
\frac{\partial r}{\partial x_{i}(p)}=-r_{s_{i}}=-\frac{x_{i}(q)-x_{i}(p)}{r} \\
\frac{\partial^{2} r}{\partial x_{i}(p) . \partial x_{j}(p)}=-r_{i_{i j}}=-\frac{\delta_{i j}-r_{i_{i}} \cdot r_{i_{j}}}{r}
\end{gathered}
$$

onde $\delta_{\mathrm{ij}}$ é o delta de Kronecker.

Após derivar $\mathrm{x}_{1}$ e $\mathrm{x}_{2}$, dados na eq.(2.32.a), em relação a $\mathbf{n}$, obtém-se:

$$
\frac{\partial x_{1}(p)}{\partial n}=n_{1}=\cos (\alpha)
$$




$$
\frac{\partial x_{2}(p)}{\partial n}=n_{2}=\operatorname{sen}(\alpha)
$$

Pode-se escrever explicitamente a eq.(2.91.a) como:

$$
\begin{gathered}
\frac{\partial r}{\partial x_{1}(p)}=r,,_{1}=\cos (\theta) \\
\frac{\partial r}{\partial x(p)}=r,_{2}=\operatorname{sen}(\theta)
\end{gathered}
$$

Substituindo-se (2.93) e (2.92) em (2.90), obtém-se:

$$
\frac{\partial \mathbf{r}}{\partial \mathbf{n}}=\cos (\theta) \cdot \operatorname{sen}(\alpha)+\operatorname{sen}(\theta) \cdot \operatorname{sen}(\alpha)=\cos (\beta)
$$

Logo, a eq.(2.89) fica:

$$
\frac{\partial w^{*}}{\partial \mathrm{n}}=\frac{\mathrm{r}}{4 \cdot \pi \cdot \mathrm{D}} \cdot \ln (\mathrm{r}) \cdot \cos (\beta)
$$

ou

$$
\frac{\partial w^{*}}{\partial \mathrm{n}}=\frac{r}{4 \cdot \pi \cdot \mathrm{D}} \cdot \ln (r) \cdot\left(r, n_{i} \cdot n_{i}\right)
$$

Obtêm-se, ainda, as equações:

$$
\begin{gathered}
M_{n}^{*}=-\frac{1}{4 \cdot \pi} \cdot\left[(1+v) \cdot \ln (r)+(1-v) \cdot\left(r_{,_{i}} \cdot n_{i}\right)^{2}+v\right] \\
M_{n s}^{*}=-\frac{1}{4 \cdot \pi} \cdot(1-v) \cdot\left(r, r_{i} \cdot n_{i}\right) \cdot\left(r,,_{j} \cdot s_{j}\right) \\
V_{n}^{*}=\frac{r_{,} \cdot n_{i}}{4 \cdot \pi \cdot r} \cdot\left[2 \cdot(1-v) \cdot\left(r, r_{j} \cdot s_{j}\right)^{2}-3+v\right]+\frac{1-v}{4 \cdot \pi \cdot R} \cdot\left[1-2 \cdot\left(r,,_{i} \cdot s_{i}\right)^{2}\right]
\end{gathered}
$$


onde:

$\mathbf{r}_{\mathbf{i}} \cdot \mathbf{n}_{\mathbf{i}}=\cos (\beta)$

$-\mathbf{r},{ }_{i} \cdot s_{i}=\operatorname{sen}(\beta)$

Conforme se verá no próximo capítulo, é também necessário determinar a solução fundamental da derivada do deslocamento fundamental e dos esforços definidos no ponto “q" em relação ao sistema $(\mathbf{m}, \mathbf{u})$, figura 2.32 .



FIGURA 2.32 - Sistema de Coordenadas $(n, s)$ e $(m, u)$.

Analogamente a (2.89), (2.90) e (2.91) obtém-se:

$$
\begin{gathered}
\frac{\partial w^{*}}{\partial \mathrm{m}}=\frac{\mathbf{d} w^{*}}{\mathrm{dr}} \cdot \frac{\partial \mathbf{r}}{\partial \mathrm{m}} \\
\frac{\partial \mathrm{r}}{\partial \mathrm{m}}=\frac{\partial \mathrm{r}}{\partial \mathrm{x}_{\mathbf{i}}(\mathbf{q})} \cdot \frac{\partial \mathrm{x}_{\mathbf{i}}(\mathbf{q})}{\partial \mathrm{m}} \\
\frac{\partial \mathrm{r}}{\partial \mathbf{x}_{\mathbf{i}}(\mathbf{q})}=-\mathbf{r}_{\mathbf{i}}=-\frac{\mathbf{x}_{\mathbf{i}}(\mathbf{p})-\mathbf{x}_{\mathbf{i}}(\mathbf{q})}{\mathbf{r}}
\end{gathered}
$$




$$
\frac{\partial^{2} \mathbf{r}}{\partial \mathbf{x}_{\mathbf{i}}(\mathbf{q}) \cdot \partial \mathbf{x}_{\mathbf{j}}(\mathbf{q})}=-\mathbf{r},_{\mathrm{ij}}=-\frac{\delta_{\mathrm{ij}}-\mathbf{r},_{\mathbf{i}} \cdot \mathbf{r},{ }_{\mathbf{j}}}{\mathbf{r}}
$$

Com a ajuda da transformação (2.32.a), o sistema de coordenadas $(\mathbf{m}, \mathbf{u})$ mostrado na figura 2.32, obtém-se as derivadas de $\mathbf{x}_{\mathbf{1}}(\mathbf{q})$ e $\mathbf{x}_{\mathbf{2}}(\mathbf{q})$ em relação a $\mathbf{m}$, dadas por:

$$
\begin{aligned}
& \frac{\partial \mathbf{x}_{1}(\mathbf{q})}{\partial \mathrm{m}}=\mathrm{m}_{1}=\cos (\gamma) \\
& \frac{\partial \mathrm{x}_{2}(\mathbf{q})}{\partial \mathrm{m}}=\mathrm{m}_{2}=\operatorname{sen}(\gamma)
\end{aligned}
$$

Pode-se escrever explicitamente (2.102.a) como:

$$
\begin{aligned}
& \frac{\partial r}{\partial x_{1}(q)}=-r,_{1}=-\cos (\theta) \\
& \frac{\partial r}{\partial x_{2}(q)}=-r,{ }_{2}=-\operatorname{sen}(\theta)
\end{aligned}
$$

Substituindo-se (2.103) e (2.104) em (2.101), obtém-se:

$$
\frac{\partial r}{\partial m}=-\cos (\theta) \cdot \cos (\gamma)-\operatorname{sen}(\theta) \cdot \operatorname{sen}(\gamma)=-\cos (\phi)
$$

Portanto, (2.100) fica:

$$
\frac{\partial w^{*}}{\partial \mathrm{m}}=-\frac{r}{4 \cdot \pi \cdot \mathrm{D}} \cdot \ln (\mathrm{r}) \cdot \cos (\phi)
$$

Em função dos versores $\overrightarrow{\mathbf{r}}$ e $\overrightarrow{\mathbf{m}},(2.106)$ pode ser escrita como:

$$
\frac{\partial w^{*}}{\partial \mathrm{m}}=-\frac{\mathrm{r}}{4 \cdot \pi \cdot \mathrm{D}} \cdot \ln (\mathrm{r}) \cdot(\overrightarrow{\mathrm{r}} \cdot \overrightarrow{\mathrm{m}})
$$


2 RELAÇÖES BÁSICAS: TEORIA DA ELASTICIDADE E PLACAS

54

ou indicialmente:

$$
\frac{\partial w^{*}}{\partial \mathrm{m}}=-\frac{\mathbf{r}}{4 \cdot \pi \cdot \mathrm{D}} \cdot \ln \left(\mathbf{r},{ }_{\mathrm{i}} \cdot \mathrm{m}_{\mathrm{i}}\right)
$$

Para as demais soluções fundamentais, derivadas em relação a m, obtém-se:

$$
\begin{aligned}
& \frac{\partial}{\partial m}\left(\frac{\partial w^{*}}{\partial m}\right)=-\frac{1}{4 \cdot \pi \cdot D} \cdot\left[\left(r_{i} \cdot m_{i}\right) \cdot\left(r, j \cdot m_{j}\right)+\left(n_{j} \cdot m_{j}\right) \cdot \ln (r)\right] \\
& \frac{\partial \mathbf{M}_{n}^{*}}{\partial m}=\frac{1}{4 \cdot \pi \cdot D} \cdot\left\{(1+v) \cdot\left(r_{, j} \cdot m_{j}\right)+2 \cdot(1-v) \cdot r_{i} \cdot n_{i} \cdot\left[\left(n_{j} \cdot m_{j}-r_{,} \cdot n_{i}\right) \cdot\left(r,,_{j} \cdot m_{j}\right)\right]\right\}
\end{aligned}
$$

$$
\frac{\partial \mathbf{M}_{n s}^{*}}{\partial m}=\frac{1-v}{4 \cdot \pi \cdot D} \cdot\left[\left(r_{i} \cdot n_{i}\right) \cdot\left(s_{j} \cdot m_{j}\right)-2 \cdot\left(r, m_{j} \cdot m_{j}\right) \cdot\left(r_{,_{i}} \cdot s_{i}\right) \cdot\left(r_{,_{i}} \cdot n_{i}\right)+\left(r_{r_{i}} \cdot s_{i}\right) \cdot\left(n_{j} \cdot m_{j}\right)\right]
$$

$$
\begin{aligned}
& \frac{\partial V_{n}^{*}}{\partial m}=\frac{1}{4 \cdot \pi \cdot r^{2}} \cdot\left\{2 \cdot ( 1 - v ) \cdot ( r , , _ { i } \cdot s _ { i } ) \cdot \left[\left(r,,_{i} \cdot s_{i}\right) 4 \cdot\left(r, n_{i} \cdot n_{i}\right) \cdot\left(r, r_{i} \cdot s_{i}\right) \cdot\left(r, j \cdot m_{j}\right)\right.\right. \\
& -2 \cdot\left(r_{1}, n_{1}\right) \cdot\left(s_{j} \cdot m_{j}\right)-\left(r, s_{1}\right) \cdot\left(n_{j} \cdot m_{j}\right)+(3-v) \cdot\left[\left(n_{1} \cdot m_{j}\right)-2 \cdot\left(r, n_{1}\right) \cdot\right. \\
& \left.\left.\cdot\left(r_{, j} \cdot m_{j}\right)\right]\right\}+\frac{i-v}{R \cdot \pi \cdot r} \cdot\left(r_{y_{i}} \cdot s_{i}\right) \cdot\left[\left(s_{j} \cdot m_{j}\right)-\left(r_{i} \cdot s_{i}\right) \cdot\left(r_{y_{j}} \cdot m_{j}\right)\right]
\end{aligned}
$$




\section{EQUAÇÕES INTEGRAIS PARA FLEXÃO DE PLACA}

\subsection{Introdução}

Neste capítulo são desenvolvidas as equações integrais de placas, baseadas na Teoria Clássica de Kirchhoff, necessária à formulação do problema pelo Método dos Elementos de Contorno. As equações integrais têm incógnita no integrando, assim como as equações diferenciais têm incógnitas funções que contenha pelo menos uma derivada.

Inicialmente, são apresentadas as equações integrais básicas para placas com espessura constante submetida a carregamento distribuído e, em seguida, para placas com espessura variável.

As equações integrais necessárias à formulação do MEC envolvem soluções fundamentais. A obtenção destas equações pode ser feita utilizando o método dos resíduos ponderados ou a partir do teorema da reciprocidade de Betti que é o procedimento adotado neste trabalho.

Aplicando-se o teorema de Betti a uma placa submetida a dois carregamentos não simultâneos $\mathbf{g}$ e $\mathbf{g}^{*}$, que dão origem a dois estados de tensão e deslocamentos distintos, obtém-se, após a integração da expressão obtida pelo teorema, chegar-se-á às equações integrais, que relacionam o deslocamento $w(q)$ de um ponto qualquer do domínio da placa com os deslocamentos e forças de superficie ao longo do contorno.

Determina-se também, a equação da derivada direcional do deslocamento, $\partial w(q) / \partial m$, que pode também ser empregada na formulação do método, veja-se PAIVA (1987). 


\subsection{Equação Integral de um Ponto do Domínio da Placa com Espessura Constante}

Seja uma placa isótropa qualquer de espessura constante $\mathbf{t}$, contorno $\Gamma \mathrm{e}$ domínio $\Omega$, em equilíbrio, contida numa placa infinita de contorno $\Gamma_{\infty}$ e de domínio $\Omega_{\infty}$, da qual ela é apenas um subdomínio, conforme mostrada na figura 3.1 .

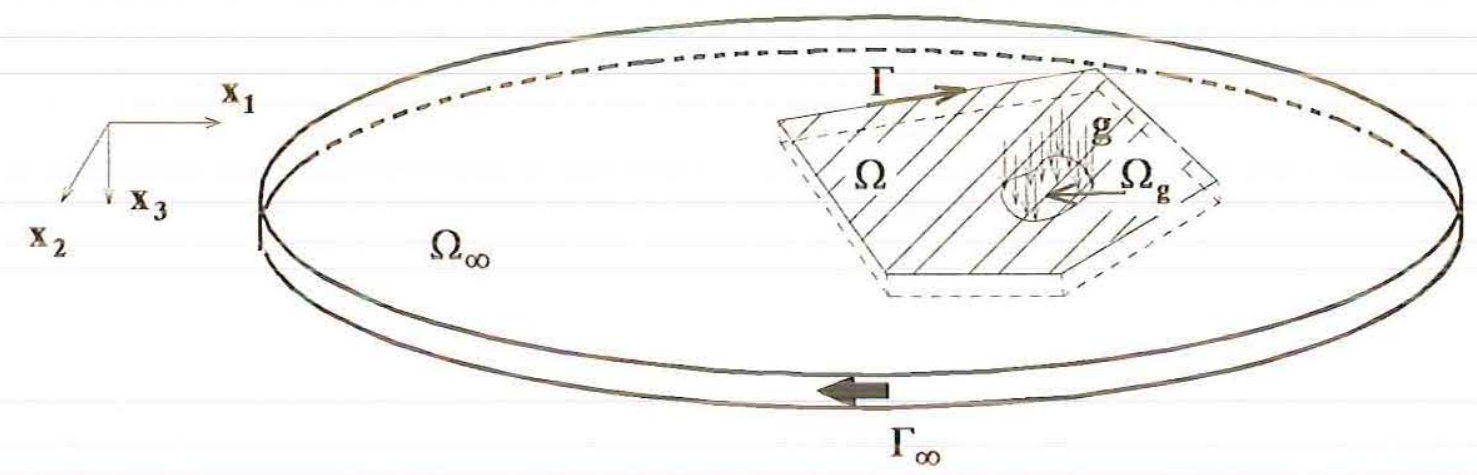

FIGURA 3.1 - Placa Finita Contida em uma Placa Infinita, $\Omega \in \Omega_{\infty}$.

Todos os campos do problema real estão definidos em $\Omega$ e todos os valores de * estão definidos em $\Omega_{\omega}$. Considere-se então, dois carregamentos não simultâneos g, definido na parte finita, e $\mathbf{g}^{*}$, pode ser definido em todo parte, onde o símbolo * é utilizado para definir o carregamento e a solução fundamental. Assim, associados a estes carregamentos, têm-se as superficies elásticas $w$ e $w^{*}$, os estados de tensões $\sigma_{i j}$ e $\sigma_{i j}^{*}$ e seus respectivos estados de deformações $\varepsilon_{i j}$ e $\varepsilon_{i j}^{*}$, figura 3.2. Pode-se então, escrever o teorema da reciprocidade de BETTI (1872) da seguinte forma:

$$
\int_{V} \sigma_{i j}^{*} \cdot \varepsilon_{i j} \cdot d V=\int_{V} \sigma_{i j} \cdot \varepsilon_{i j}^{*} \cdot d V \quad(i, j=1,2,3)
$$


onde

$$
\sigma_{\mathrm{ij}} \cdot \varepsilon_{\mathrm{ij}}^{*}=\sigma_{11} \cdot \varepsilon_{11}^{*}+\sigma_{22} \cdot \varepsilon_{22}^{*}+\sigma_{33} \cdot \varepsilon_{33}^{*}+2 \cdot\left(\sigma_{12} \cdot \varepsilon_{12}^{*}+\sigma_{13} \cdot \varepsilon_{13}^{*}+\sigma_{23} \cdot \varepsilon_{23}^{*}\right)
$$

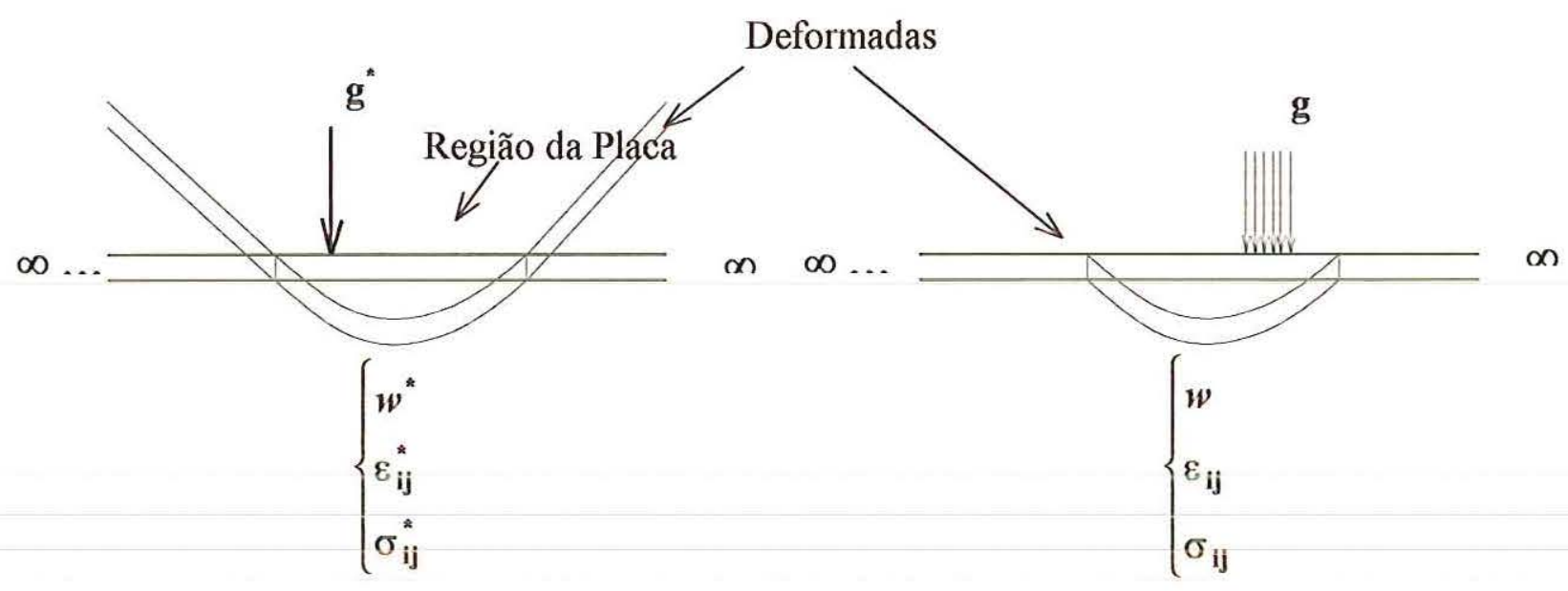

FIGURA 3.2 - Estados de Carregamentos Virtual $\mathbf{g}^{*}$ e Real g.

Denominando-se $\mathbf{U}_{2}$ o termo da direita da eq.(3.1) e desprezando-se as deformações por cisalhamento transversal e as tensões relativas à direção normal ao plano da placa, obtém-se:

$$
U_{2}=\int_{V} \sigma_{i j}, \varepsilon_{i j}^{*} \cdot d V \quad(i, j=1,2)
$$

Substituindo-se em (3.3) a expressão dada pela eq.(2.12), resulta:

$$
U_{2}=\iint_{\Omega}\left[\int_{x_{3}}-\sigma_{i j} \cdot x_{3} \cdot w_{, i_{j}}^{*} \cdot d x_{3}\right] \cdot d \Omega
$$

Integrando-se (3.4) ao longo da espessura da placa, isto é, em relação a $\mathbf{x}_{3}, \mathrm{e}$ considerando a eq.(2.14), a eq.(3.4) transforma-se em uma integral sobre o domínio $\Omega$, dada por:

$$
U_{2}=\int_{\Omega}-m_{i j} \cdot w_{9, j}^{*} \cdot d \Omega \quad(i, j=1,2)
$$


Aplicando-se o teorema da divergência, ou integrando-se (3.5) por partes, em relação às coordenadas $\mathbf{x}_{\mathbf{j}}$, obtém-se:

$$
\mathrm{U}_{2}=-\int_{\Gamma} \mathrm{m}_{\mathrm{ij}} \cdot w_{, \mathrm{i}}^{*} \cdot \mathrm{n}_{\mathrm{j}} \cdot \mathrm{d} \Gamma+\int_{\Omega} \mathrm{m}_{\mathrm{ij}, \mathrm{j}} \cdot w_{9 \mathrm{i}}^{*} \cdot \mathrm{d} \Omega
$$

onde $\mathbf{n}_{\mathbf{j}}$ são os co-senos diretores do versor normal ao contorno da placa.

Conforme a figura (3.3), os co-senos diretores são:

$$
\begin{aligned}
& \mathbf{n}_{1}=\cos (\alpha) \\
& \mathbf{n}_{2}=\operatorname{sen}(\alpha)
\end{aligned}
$$

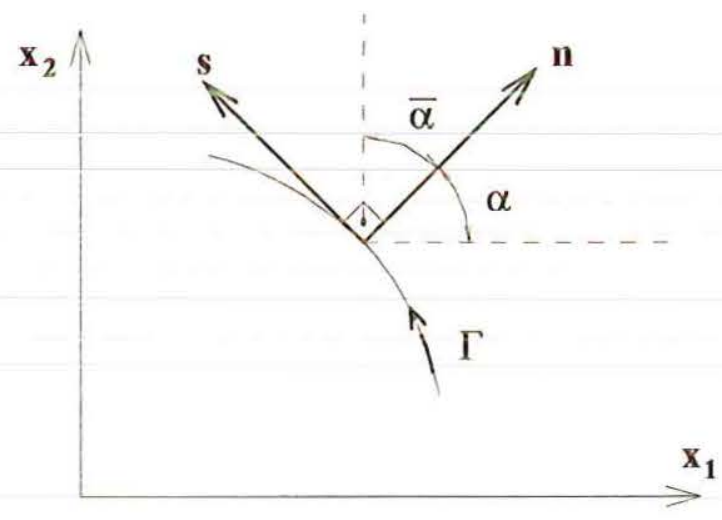

FIGURA 3.3 - Sistema de Coordenadas (n,s), Normal e Tangente ao Contorno.

Integrando-se novamente por partes a segunda parcela da eq.(3.6), a expressão de $\mathbf{U}_{2}$ fica:

$$
\mathrm{U}_{2}=-\int_{\Gamma} \mathrm{m}_{\mathrm{ij}} \cdot w_{i_{\mathrm{i}}}^{*} \cdot \mathrm{n}_{\mathrm{j}} \cdot \mathrm{d} \Gamma+\int_{\Gamma} \mathrm{m}_{\mathrm{ij}, \mathrm{j}} \cdot w^{*} \cdot \mathrm{n}_{\mathrm{i}} \mathrm{d} \Gamma-\int_{\Omega} \mathrm{m}_{\mathrm{ij}, \mathrm{ij}} \cdot w^{*} \cdot \mathrm{d} \Omega
$$

Considerando-se a equação diferencial de placas em função dos momentos (2.26), equação de equilíbrio (2.21) e equação (2.37), obtém-se para $\mathbf{U}_{2}$ a seguinte expressão: 


$$
\mathrm{U}_{2}=-\int_{\Gamma} \mathrm{m}_{\mathrm{ij}} \cdot w_{,}^{*} \cdot \mathrm{n}_{\mathrm{j}} \cdot \mathrm{d} \Gamma+\int_{\Gamma} \mathrm{q}_{\mathrm{n}} \cdot w^{*} \cdot \mathrm{d} \Gamma+\int_{\Omega} \mathrm{g} \cdot w^{*} \cdot \mathrm{d} \Omega
$$

As derivadas de $w^{*}$ em relação às coordenadas $\mathbf{x}_{\mathbf{i}}$ são:

$$
w_{,}^{*}=\frac{\partial w^{*}}{\partial \mathbf{x}_{\mathbf{i}}}=\frac{\partial w^{*}}{\partial \mathbf{n}} \cdot \frac{\partial \mathbf{n}}{\partial \mathbf{x}_{\mathbf{i}}}+\frac{\partial w^{*}}{\partial \mathrm{s}} \cdot \frac{\partial \mathbf{s}}{\partial \mathbf{x}_{\mathbf{i}}}
$$

ou seja,

$$
w_{i}^{*}=\frac{\partial w^{*}}{\partial \mathbf{n}} \cdot n_{i}+\frac{\partial w^{*}}{\partial s} \cdot s_{1}
$$

sendo $s_{\mathbf{i}}$ os co-senos diretores do versor tangente ao contorno, conforme a figura 3.3.

Substituindo-se (3.10) na primeira parcela do segundo membro da equação (3.8), tem-se:

$$
\mathbf{U}_{2}=-\int_{\Gamma}\left(\mathbf{m}_{\mathrm{ij}} \cdot \mathbf{n}_{\mathrm{i}} \cdot \mathrm{n}_{\mathrm{j}} \cdot \frac{\partial w^{*}}{\partial \mathrm{n}}+\mathrm{m}_{\mathrm{ij}} \cdot \mathrm{s}_{\mathrm{i}} \cdot \mathrm{n}_{\mathrm{j}} \cdot \frac{\partial w^{*}}{\partial \mathrm{s}}\right) \mathrm{d} \Gamma+\int_{\Gamma} \mathbf{q}_{\mathrm{n}} \cdot w^{*} \cdot \mathrm{d} \Gamma+\int_{\Omega} \mathrm{g} \cdot w^{*} \cdot \mathrm{d} \Omega
$$

Considerando as equações (2.31.a) e (2.31.c) pode-se escrever que:

$$
\mathbf{U}_{2}=-\int_{\Gamma}\left(\mathbb{M}_{n} \cdot \frac{\partial w^{*}}{\partial \mathbf{n}}+\mathbf{M}_{n s} \cdot \frac{\partial w^{*}}{\partial s}-\mathbf{q}_{n} \cdot w^{*}\right) \mathrm{d} \Gamma+\int_{\Omega} g \cdot w^{*} \cdot d \Omega
$$

Integrando-se por partes o segundo termo da integral sobre o contorno que figura no segundo membro da eq(3.12), obtém-se:

$$
\int_{\Gamma}\left(\mathbf{M}_{\mathrm{ns}} \cdot \frac{\partial w^{*}}{\partial \mathrm{s}}\right) \cdot \mathrm{d} \Gamma=\left[\mathbf{M}_{\mathrm{ns}} \cdot w^{*}\right]_{\Gamma_{1}}^{\Gamma_{2}}-\int_{\Gamma} \frac{\partial \mathrm{M}_{\mathrm{ns}}}{\partial \mathrm{s}} \cdot w^{*} \cdot \mathrm{d} \Gamma
$$

onde $\Gamma_{1}$ e $\Gamma_{2}$ representam os limites do contorno no qual se realiza a integração. 
A primeira parcela do segundo membro da relação (3.13) é nula para o caso de um contorno fechado cuja representação paramétrica e a respectiva derivada sejam contínuas. Caso contrário, ela dará origem a reação nas angulosidades ou cantos no contorno da placa.

Pode-se escrever esta parcela sobre todos os lados do contorno, em função da reação $\mathbf{R}_{\mathbf{c i}}$ de cada canto genérico $\mathbf{i}$, indicado na figura 3.4 , a qual é dada por:

$$
\mathbf{R}_{\mathrm{ci}}=\mathbf{M}_{\mathrm{nsi}}^{+}-\mathbf{M}_{\mathrm{nsi}}^{-}
$$

sendo $\mathbf{M}_{\mathbf{n s i}}^{+}$e $\mathbf{M}_{\mathbf{n s i}}^{-}$, respectivamente, os momentos volventes posterior e anterior ao canto i, figura 3.4. Deve-se observar que o sentido do momento volvente anterior foi alterado, figura 3.4, resultando no sinal negativo na eq.(3.14).

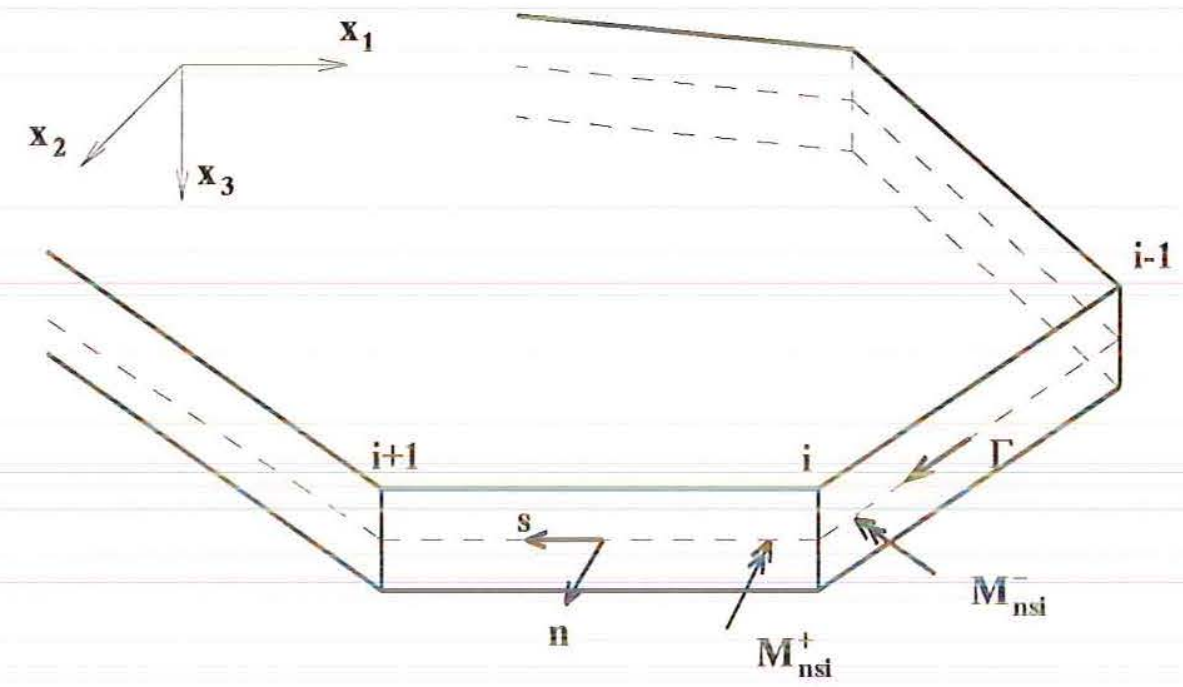

FIGURA 3.4 - Momentos Volventes em um Canto i da Placa.

Considerando (3.14), a equação (3.13) pode ser escrita como:

$$
\int_{\Gamma}\left(\mathbf{M}_{\mathrm{ns}} \cdot \frac{\partial w^{*}}{\partial \mathrm{s}}\right) \cdot \mathrm{d} \Gamma=-\sum_{\mathrm{i}=1}^{\mathrm{N}_{\mathrm{c}}} \mathbf{R}_{\mathrm{ci}} \cdot w_{\mathrm{ci}}^{*}-\int_{\Gamma} \frac{\partial \mathbf{M}_{\mathrm{ns}}}{\partial \mathrm{s}} \cdot w^{*} \cdot \mathrm{d} \Gamma
$$


onde $\mathrm{N}_{\mathrm{c}}$ é o número total de cantos do contorno da placa e $w_{\mathrm{ci}}^{*}$ é o valor do deslocamento $w^{*}$ no canto $\mathbf{i}$.

Substituindo-se estas equações em (3.12), tem-se:

$$
\mathbf{U}_{2}=\int_{\Gamma}\left(\mathbf{q}_{\mathrm{n}} \cdot w^{*}+\frac{\partial \mathbf{M}_{\mathrm{ns}}}{\partial \mathrm{s}} \cdot w^{*}-\mathbf{M}_{\mathrm{n}} \cdot \frac{\partial w^{*}}{\partial \mathrm{n}}\right) \mathrm{d} \Gamma+\sum_{\mathrm{i}=1}^{\mathrm{N}_{\mathrm{c}}} \mathbf{R}_{\mathrm{ci}} \cdot w_{\mathrm{ci}}^{*}+\int_{\Omega} \mathrm{g} \cdot w^{*} \cdot \mathrm{d} \Omega
$$

Utilizando-se a definição de $\mathbf{V}_{\mathrm{n}}$, dada na eq.(2.38), e considerando-se que a carga g está distribuída em $\Omega_{\mathrm{g}}$, obtém-se:

$$
\mathbf{U}_{2}=\int_{\Gamma}\left(\mathrm{V}_{\mathrm{n}} \cdot w^{*}-\mathbf{M}_{\mathrm{n}} \cdot \frac{\partial w^{*}}{\partial \mathrm{n}}\right) \cdot \mathrm{d} \Gamma+\sum_{\mathrm{i}=1}^{N_{\mathrm{c}}} \mathbf{R}_{\mathrm{ci}} \cdot w_{\mathrm{ci}}^{*}+\int_{\Omega_{\mathrm{g}}} \mathrm{g} \cdot w^{*} \cdot \mathrm{d} \Omega_{\mathrm{g}}
$$

O termo da esquerda da eq.(3.1) pode ser desenvolvido de modo análogo, chegando-se a:

$$
U_{1}=\int_{V} \sigma_{i j}^{*} \cdot \varepsilon_{i j} \cdot d V=\int_{\Gamma}\left(V_{n}^{*} \cdot w-M_{n}^{*} \cdot \frac{\partial w}{\partial n}\right) \cdot d \Gamma+\sum_{i=1}^{N_{c}} R_{c i}^{*} \cdot w_{c i}+\int_{\Omega} g^{*} \cdot w \cdot d \Omega
$$

Finalmente, a partir das equações (3.1), (3.17) e (3.18), o teorema de Bet̂i aplicado a placas fica:

$$
\begin{aligned}
& \int_{\Gamma}\left(V_{\mathbf{n}}^{*} \cdot w-\mathbf{M}_{\mathbf{n}}^{*} \cdot \frac{\partial w}{\partial \mathbf{n}}\right) \cdot \mathbf{d} \Gamma+\sum_{\mathbf{i}=1}^{N_{\mathbf{c}}} \mathbf{R}_{\mathbf{c i}}^{*} \cdot w_{\mathbf{c i}}+\int_{\Omega} \mathrm{g}^{*} \cdot w_{\cdot} \mathbf{d} \Omega \\
& =\int_{\Gamma}\left(\mathbf{V}_{\mathrm{n}} \cdot w^{*}-\mathbf{M}_{\mathrm{n}} \cdot \frac{\partial w^{*}}{\partial \mathrm{n}}\right) \cdot \mathrm{d} \Gamma+\sum_{\mathrm{i}=1}^{N_{\mathrm{c}}} \mathbf{R}_{\mathrm{ci}} \cdot w_{\mathrm{ci}}{ }^{*}+\int_{\Omega_{\mathrm{g}}} \mathrm{g} \cdot w^{*} \cdot \mathrm{d} \Omega_{\mathrm{g}}
\end{aligned}
$$

Conforme foi mencionado, admite-se que $\mathbf{g}^{*}$ seja uma carga unitária aplicada em um ponto " $q$ " qualquer do domínio da placa ou do domínio $\Omega$ " e que sua representação matemática é a distribuição delta de Dirac, $\delta(\mathbf{q}, \mathbf{p})$, conforme (2.68). 
Assim, os deslocamentos e esforços fundamentais associados a este carregamento são funções do ponto de aplicação da carga, chamado ponto de carregamento " $q$ ", e do ponto de deslocamento "p" no domínio ou "P" se estiver no contorno da placa.

Os deslocamentos e esforços provenientes do carregamento real $\mathbf{g}$ são funções apenas do ponto "p" ou "P", onde os efeitos de g são medidos, pois a posição deste carregamento é fixa.

Substituindo-se então, $\mathbf{g}^{*}$ por $\delta(\mathbf{q}, \mathbf{p})$ em (3.19) e usando-se a representação de cada variável em função do dois pontos "q" e "p", para os valores fundamentais, e em função de um ponto apenas, em caso contrário, obtém-se:

$$
\begin{gathered}
\int_{\Omega} \delta(\mathbf{q}, \mathbf{p}) \cdot w(\mathbf{p}) \cdot \mathrm{d} \Omega(\mathbf{p})+\int_{\Gamma}\left[V_{\mathrm{n}}^{*}(\mathbf{q}, \mathbf{P}) \cdot w(\mathbf{P})-\mathbf{M}_{\mathrm{n}}^{*}(\mathbf{q}, \mathbf{P}) \cdot \frac{\partial w}{\partial \mathrm{n}}(\mathbf{P})\right] \cdot \mathrm{d} \Gamma(\mathbf{P}) \\
+\sum_{\mathrm{i}=1}^{N_{\mathrm{c}}} \mathbf{R}_{\mathrm{ci}}^{*}(\mathbf{q}, \mathbf{P}) \cdot w_{\mathrm{ci}}(\mathbf{P})=\int_{\Gamma}\left[\mathrm{V}_{\mathrm{n}}(\mathbf{P}) \cdot w^{*}(\mathbf{q}, \mathbf{P})-\mathbf{M}_{\mathrm{n}}(\mathbf{P}) \cdot \frac{\partial w^{*}}{\partial \mathrm{n}}(\mathbf{q}, \mathbf{P})\right] \cdot \mathrm{d} \Gamma(\mathbf{P}) \\
+\sum_{\mathrm{i}=1}^{N_{\mathrm{c}}} \mathbf{R}_{\mathrm{ci}}(\mathbf{P}) \cdot w_{\mathrm{ci}}^{*}(\mathbf{q}, \mathbf{P})+\int_{\Omega_{\mathrm{g}}} \mathrm{g}(\mathbf{p}) \cdot w^{*}(\mathbf{q}, \mathbf{p}) \cdot \mathrm{d} \Omega_{\mathrm{g}}(\mathbf{p})
\end{gathered}
$$

Aplicando-se a propriedade da função delta de Dirac, e considerando-se $q \in \Omega$, dada em (2.68), tem-se que:

$$
\int_{\Omega} \delta(q, p) \cdot w(p) \cdot d \Omega(p)=w(q)
$$

sendo $w(q)$ o deslocamento no ponto de carregamento, devido à carga $\mathbf{g}$.

Substituindo-se (3.21) em (3.20), obtém-se a equação:

$$
\begin{aligned}
& w(\mathrm{q})+\int_{\Gamma}\left[\mathrm{V}_{\mathrm{n}}^{*}(\mathrm{q}, \mathrm{P}) \cdot w(\mathrm{P})-\mathrm{M}_{\mathrm{n}}^{*}(\mathrm{q}, \mathrm{P}) \cdot \frac{\partial w}{\partial \mathrm{n}}(\mathrm{P})\right] \cdot \mathrm{d} \Gamma(\mathrm{P}) \\
& +\sum_{i=1}^{N_{\mathrm{c}}} \mathbf{R}_{\mathrm{ci}}^{*}(\boldsymbol{q}, \mathbf{P}) \cdot w_{\mathrm{ci}}(\mathbf{P})=\int_{\Gamma}\left[\mathbf{V}_{\mathrm{n}}(\mathbf{P}) \cdot w^{*}(\mathbf{q}, \mathbf{P})-\mathbf{M}_{\mathrm{n}}(\mathbf{P}) \cdot \frac{\partial w^{*}}{\partial \mathbf{n}}(\mathbf{q}, \mathbf{P})\right] \cdot \mathrm{d} \Gamma(\mathbf{P})
\end{aligned}
$$




$$
+\sum_{\mathrm{i}=1}^{N_{\mathrm{c}}} \mathbf{R}_{\mathrm{ci}}(\mathbf{P}) \cdot w_{\mathrm{ci}}^{*}(\mathbf{q}, \mathbf{P})+\int_{\Omega_{\mathrm{g}}} \mathrm{g}(\mathrm{p}) \cdot w^{*}(\mathbf{q}, \mathrm{p}) \cdot \mathrm{d} \Omega_{\mathrm{g}}(\mathrm{p})
$$

A equação (3.22) é a representação integral do deslocamento $w$ de um ponto " $q$ " do domínio da placa de espessura constante, envolvendo as soluções fundamentais obtidas no capítulo 2. E os valores de contorno que no caso são os deslocamentos $w(\mathbf{P}), \partial w(\mathbf{P}) / \partial \mathbf{n}$ e $w_{\mathrm{ci}}(\mathbf{P})$ e as forças de superfície $\mathbf{V}_{\mathbf{n}}(\mathbf{P}), \mathbf{M}_{\mathbf{n}}(\mathbf{P})$ e a reação de canto $\mathbf{R}_{\mathrm{d}}(\mathbf{P})$.

A partir de (3.22), pode-se obter, também, a equação integral da derivada direcional do deslocamento em relação à uma direção $\mathbf{m}$ qualquer para um ponto " $q$ " da placa, conforme indicado na figura 2.30. Derivando-se $\boldsymbol{w}$ em relação a $\mathbf{m}$, obtémse:

$$
\frac{\partial w}{\partial m}(\mathbf{q})+\int_{\Gamma}\left[\frac{\partial \mathbf{V}_{n}^{*}}{\partial m}(\mathbf{q}, \mathbf{P}) \cdot w(\mathbf{P})-\frac{\partial \mathbf{M}_{n}^{*}}{\partial m}(\mathbf{q}, \mathbf{P}) \cdot \frac{\partial w}{\partial n}(\mathbf{P})\right] \cdot \mathbf{d} \Gamma(\mathbf{P})
$$

$+\sum_{\mathrm{i}=1}^{\mathrm{N}_{\mathrm{c}}} \frac{\partial \mathbf{R}_{\mathrm{ci}}{ }^{*}}{\partial \mathbf{m}}(\mathbf{q}, \mathbb{P}) \cdot w_{\mathrm{ci}}(\mathbf{P})=\int_{\Gamma}\left\{\mathbf{V}_{\mathrm{n}}(\mathbf{P}) \cdot \frac{\partial w^{n}}{\partial \mathbf{m}}(\mathbf{q}, \mathbb{P})-\mathbf{M}_{\mathrm{n}}(\mathbf{P}) \cdot \frac{\partial}{\partial \mathbf{m}}\left[\frac{\partial w^{n}}{\partial \mathbf{n}}(\mathbf{q}, \mathbb{P})\right]\right\} \cdot \mathbf{d} \Gamma(\mathbf{P})$

$+\sum_{\mathrm{i}=1}^{N_{\mathrm{c}}} \mathbf{R}_{\mathrm{ci}}(\mathbf{P}) \cdot \frac{\partial w_{\mathrm{ci}}^{\mathrm{*}}}{\partial \mathrm{m}}(\mathbf{q}, \mathbf{P})+\int_{\Omega_{\mathrm{g}}} \mathbf{g}(\mathbf{p}) \cdot \frac{\partial w^{\mathrm{k}}}{\partial \mathrm{m}}(\mathbf{q}, \mathbf{p}) \cdot \mathrm{d} \Omega_{\mathrm{g}}(\mathbf{p})$ 


\subsection{Equação Integral de um Ponto do Domínio da Placa de Espessura Variável}

As relações integrais básicas para o problema de flexão de placas são usualmente deduzidas considerando-se a teoria da Reciprocidade de Betti, como foi visto no item 3.2, que pode ser expressa em termos de tensão e deformação, como na eq.(3.1). A expressão da reciprocidade também pode ser escrita para o problema de flexão de placas utilizando-se curvaturas e momentos como na eq.(3.5).

Para um ponto de um sólido, segundo o teorema de Betti, a seguinte expressão é válida:

$$
w_{i \mathrm{ij}} \cdot \mathrm{m}_{\mathrm{ij}}^{*}=w_{\mathrm{ij}}^{*} \cdot \mathrm{m}_{\mathrm{ij}}
$$

Através da eq.(2.16) e utilizando-se do artifício de multiplicar e dividir o

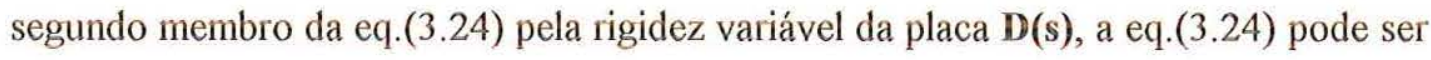
rescrita, como:

$$
\begin{aligned}
w_{9 \mathrm{ij}} \cdot\left[-\mathbf{D}_{0} \cdot\left(v \cdot \delta_{\mathrm{ij}} \cdot w_{9 \mathrm{kk}}^{*}+(1-v) \cdot w_{9 \mathrm{ij}}{ }^{*}\right)\right] \\
=w_{9 \mathrm{ij}}^{*} \cdot\left[-\mathbf{D}_{0} \cdot\left(v \cdot \delta_{\mathrm{ij}} \cdot w_{9 \mathbf{k k}}+(1-v) \cdot w_{9 \mathrm{ij}}\right)\right] \cdot \frac{\mathbf{D}(\mathrm{s})}{\mathbf{D}(\mathrm{s})}
\end{aligned}
$$

Fazendo-se manipulações algébricas chaga-se a:

$$
w_{9_{\mathrm{ij}}}^{*} \cdot \mathrm{m}_{\mathrm{ij}}(\mathrm{s})=\frac{\mathrm{D}(\mathrm{s})}{\mathrm{D}_{0}} \cdot w_{9_{\mathrm{ij}}} \cdot \mathrm{m}_{\mathrm{ij}}^{*}
$$


onde $\mathbf{D}(\mathbf{s})=\frac{\mathbf{E} \cdot \mathbf{t}^{3}(\mathbf{s})}{12 \cdot\left(1-v^{2}\right)}$, que varia de ponto a ponto em função da espessura $\mathbf{t}(\mathbf{s})$.

$\mathrm{O}$ artificio utilizado para obter a expressão do momento com variação de espessura $\mathbf{m}_{\mathbf{i j}}(\mathbf{s})$ é interessante, pois a solução fundamental continua sendo expressa em função de uma rigidez constante $\mathbf{D}_{\mathbf{0}}$.

Integrando-se no domínio $\Omega$ a eq.(3.26), resulta:

$$
\int_{\Omega} w_{9_{\mathrm{ij}}}^{*} \cdot \mathrm{m}_{\mathrm{ij}}(\mathrm{s}) \cdot \mathrm{d} \Omega=\int_{\Omega} \frac{\mathrm{D}(\mathrm{s})}{\mathrm{D}_{0}} \cdot w_{{ }_{\mathrm{ij}}} \cdot \mathrm{m}_{\mathrm{ij}}^{*} \cdot \mathrm{d} \Omega
$$

Fazendo $\mathbf{U}_{1}$ igual a:

$$
\mathrm{U}_{1}=\int_{\Omega} w_{,{ }_{\mathrm{ij}}}^{*} \cdot \mathrm{m}_{\mathrm{ij}}(\mathrm{s}) \cdot \mathrm{d} \Omega
$$

cuja integral pode ser representada através eq.(3.17) após integrações por parte, podendo afirmar que:

$$
\mathbf{U}_{1}=\int_{1}\left(-\mathbf{V}_{n} \cdot w^{*}+M_{n} \cdot \frac{\partial w^{*}}{\partial \mathbf{n}}\right) \cdot d \Gamma-\sum_{i=1}^{N_{c}} \mathbf{R}_{\mathrm{ci}} \cdot w_{\mathrm{ci}}^{*}-\int_{\Omega_{\mathrm{g}}} \mathrm{g} \cdot w^{*} \cdot d \Omega_{\mathrm{g}}
$$

Considerando-se agora o segundo membro da eq.(3.27), dado por:

$$
U_{2}=\int_{\Omega} \frac{D(s)}{D_{0}} m_{i j}^{*} \cdot w_{9 \mathrm{ij}} \cdot d \Omega \quad(i, j=1,2)
$$

Integrando-se (3.30) por partes, em relação às coordenadas $\mathbf{x}_{\mathbf{j}}$, obtém-se:

$$
\mathrm{D}_{0} \cdot \mathbf{U}_{2}=\int_{\Gamma} \mathrm{m}_{\mathrm{ij}}^{*} \cdot w_{9_{\mathrm{i}}} \cdot \mathbf{n}_{\mathrm{j}} \cdot \mathrm{D}(\mathrm{s}) \cdot \mathrm{d} \Gamma-\int_{\Omega} w_{\mathbf{i}_{\mathrm{i}}} \cdot\left(\mathrm{D}(\mathrm{s}) \cdot \mathrm{m}_{\mathrm{ij}, \mathrm{j}}^{*} \cdot \frac{\partial \mathrm{D}(\mathrm{s})}{\partial \mathbf{x}_{\mathbf{j}}} \mathbf{m}_{\mathrm{ij}}^{*}\right) \cdot \mathrm{d} \Omega
$$


onde $\mathbf{n}_{\mathbf{j}}$ são os co-senos diretores do versor normal ao contorno da placa, conforme a figura 3.3.

Integrando-se novamente por partes a integral de domínio da eq.(3.31), resulta:

$$
\begin{aligned}
& \mathrm{D}_{0} \cdot \mathrm{U}_{2}=\int_{\Gamma} w_{9_{\mathrm{i}}} \cdot \mathrm{n}_{\mathrm{j}} \cdot \mathrm{D}(\mathrm{s}) \cdot \mathrm{m}_{\mathrm{ij}}^{*} \cdot \mathrm{d} \Gamma-\int_{\Gamma} \mathrm{m}_{\mathrm{ij}, \mathrm{j}}^{*} \cdot w \cdot \mathbf{n}_{\mathrm{i}} \cdot \mathrm{D}(\mathrm{s}) \cdot \mathrm{d} \Gamma-\int_{\Gamma} \mathrm{m}_{\mathrm{ij}}^{*} \cdot w_{\cdot} \cdot \mathrm{n}_{\mathrm{i}} \cdot \frac{\partial \mathrm{D}(\mathrm{s})}{\partial \mathrm{x}_{\mathrm{j}}} \cdot \mathrm{d} \Gamma \\
& +\int_{\Omega} w \cdot\left(\frac{\partial \mathrm{D}(\mathrm{s})}{\partial \mathrm{x}_{\mathrm{i}}} \cdot \mathrm{m}_{\mathrm{ij}, \mathrm{j}}^{*}\right) \cdot \mathrm{d} \Omega+\int_{\Omega} w \cdot \mathrm{D}(\mathrm{s}) \cdot \mathrm{m}_{\mathrm{ij}, \mathrm{ji}}^{*} \cdot \mathrm{d} \Omega \\
& +\int_{\Omega} w \cdot \frac{\partial^{2} \mathbf{D}(\mathrm{s})}{\partial \mathbf{x}_{\mathbf{i}} \cdot \partial \mathbf{x}_{\mathbf{j}}} \cdot \mathrm{m}_{\mathrm{ij}}^{*} \cdot \mathrm{d} \Omega+\int_{\Omega} w \cdot \frac{\partial \mathbf{D}(\mathrm{s})}{\partial \mathbf{x}_{\mathbf{j}}} \cdot \mathrm{m}_{\mathrm{ij}, i}^{*} \cdot \mathrm{d} \Omega
\end{aligned}
$$

Sendo as derivadas de $w$ em relação às coordenadas $\mathrm{x}_{\mathbf{i}}$ dadas pelas relações (3.10) e considerando a equação diferencial de placas em função dos momentos (2.24), a equação de equilíbrio (2.19) e as equações (2.32), pode-se escrever a eq.(3.32) como:

$$
\begin{aligned}
& \mathbf{D}_{0} \cdot \mathbf{U}_{2}=\int_{\Gamma} \frac{\partial w}{\partial \mathbf{n}} \cdot \mathbf{D}(s) \cdot \mathbf{M}_{\mathrm{n}}^{*} \cdot \mathbf{d} \Gamma+\int_{\Gamma} \frac{\partial w}{\partial s} \cdot \mathbf{D}(s) \cdot \mathbf{M}_{\overline{\mathrm{n}}}^{*} \cdot \mathbf{d} \Gamma-\int_{\Gamma} w \cdot \mathbf{D}(\mathrm{s}) \cdot \mathbf{q}_{\mathrm{n}}^{*} \cdot \mathbf{d} \Gamma \\
& -\int_{\Gamma} w \cdot \frac{\partial \mathrm{D}(\mathrm{s})}{\partial \mathrm{x}_{\mathrm{j}}} \mathbf{m}_{\mathrm{ij}}^{*} \cdot \mathbf{n}_{\mathrm{i}} \cdot \mathrm{d} \Gamma+\int_{\Omega} w \cdot \frac{\partial \mathrm{D}(\mathrm{s})}{\partial \mathrm{x}_{\mathrm{i}}} \cdot \mathrm{q}_{\mathrm{i}}^{*} \cdot \mathrm{d} \Omega-\int_{\Omega} w \cdot \mathrm{D}(\mathrm{s}) \cdot \mathrm{g}^{*} \cdot \mathrm{d} \Omega \\
& +\int_{\Omega} w \cdot \frac{\partial^{2} \mathrm{D}(\mathrm{s})}{\partial \mathbf{x}_{\mathbf{i}} \cdot \partial \mathbf{x}_{\mathbf{j}}} \cdot \mathrm{m}_{\mathrm{ij}}^{*} \cdot \mathrm{d} \Omega+\int_{\Omega} w \cdot \frac{\partial \mathrm{D}(\mathrm{s})}{\partial \mathbf{x}_{\mathbf{j}}} \cdot \mathrm{q}_{\mathrm{j}}^{*} \cdot \mathrm{d} \Omega
\end{aligned}
$$

Integrando-se por partes a segunda integral da eq.(3.33), obtém-se:

$$
\int_{\Gamma} \mathbf{M}_{\mathrm{ns}}^{*} \cdot \frac{\partial w}{\partial \mathrm{s}} \cdot \mathbf{D}(\mathbf{s}) \cdot \mathbf{d} \Gamma=\left[\mathbf{M}_{\mathrm{ns}}^{*} \cdot w \cdot \mathbf{D}(\mathrm{s})\right]_{\Gamma_{1}}^{\Gamma_{2}}-\int_{\Gamma} w \cdot\left(\mathbf{D}(\mathrm{s}) \cdot \frac{\partial \mathbf{M}_{\mathrm{ns}}^{\mathrm{*}}}{\partial \mathrm{s}}+\frac{\partial \mathbf{D}(\mathrm{s})}{\partial \mathrm{s}} \cdot \mathbf{M}_{\mathrm{ns}}^{*}\right) \cdot \mathrm{d} \Gamma
$$


onde $\Gamma_{1}$ e $\Gamma_{2}$ representam os limites do contorno no qual se realiza a integração.

Do mesmo modo que no item (3.2), a primeira parcela do segundo membro da eq.(3.34) é nula para o caso de um contorno fechado cuja representação paramétrica e respectiva derivada sejam contínuas. Caso contrário, ela dará origem a reação nas angulosidades ou cantos no contorno da placa.

Considerando-se a eq.(3.14), a eq.(3.34) pode ser escrita como:

$$
\int_{\Gamma} \mathbf{M}_{\mathrm{ns}}^{*} \cdot \frac{\partial w}{\partial \mathrm{s}} \cdot \mathbf{D}(\mathrm{s}) \cdot \mathrm{d} \Gamma=-\sum_{\mathrm{i}=1}^{\mathrm{N}_{\mathrm{c}}} \mathbf{R}_{\mathrm{ci}}^{*} \cdot w_{\mathrm{ci}} \cdot \mathbf{D}_{\mathrm{ci}}-\int_{\Gamma} w \cdot\left(\mathbf{D}(\mathrm{s}) \cdot \frac{\partial \mathbf{M}_{\mathrm{ns}}^{\mathrm{n}}}{\partial \mathrm{s}}+\frac{\partial \mathbf{D}(\mathrm{s})}{\partial \mathrm{s}} \cdot \mathbf{M}_{\mathrm{ns}}^{*}\right) \cdot \mathrm{d} \Gamma
$$

Substituindo-se a eq.(3.35) na eq.(3.33), resulta:

$$
\begin{aligned}
& \mathbf{D}_{0} \cdot \mathbf{U}_{2}=\int_{\Gamma} \frac{\partial w}{\partial \mathbf{n}} \cdot \mathbf{D}(\mathrm{s}) \cdot \mathbf{M}_{\mathrm{n}}^{\mathrm{n}} \cdot \mathbf{d} \Gamma-\sum_{\mathrm{i}=1}^{N_{\mathrm{c}}} \mathbf{R}_{\mathrm{ci}}^{*} \cdot w_{\mathrm{ci}} \cdot \mathrm{D}_{\mathrm{ci}}-\int_{\Gamma} w \cdot \mathrm{D}(\mathrm{s}) \cdot \mathbf{q}_{\mathrm{n}}^{*} \cdot \mathbf{d} \Gamma-\int_{\Gamma} w \cdot \mathbf{D}(\mathrm{s}) \cdot \frac{\partial \mathrm{M}_{\mathrm{ns}}}{\partial \mathrm{s}} \cdot \mathbf{d} \Gamma \\
& -\int_{\Gamma} w_{0} \frac{\partial \mathrm{D}(\mathrm{s})}{\partial \mathrm{s}} \mathbf{M}_{\mathrm{ns}}^{*} \cdot \mathbf{d} \Gamma-\int_{\Gamma} w \cdot \frac{\partial \mathrm{D}(\mathrm{s})}{\partial \mathrm{x}_{\mathrm{j}}} \mathbf{m}_{\mathrm{ij}}^{*} \cdot \mathbf{n}_{\mathrm{i}} \cdot \mathbf{d} \Gamma+\int_{\Omega} w \cdot \frac{\partial \mathrm{D}(\mathrm{s})}{\partial \mathrm{x}_{\mathrm{i}}} \cdot \mathbf{q}_{\mathrm{i}}^{*} \cdot \mathbf{d} \Omega-\int_{\Omega} w \cdot \mathrm{D}(\mathrm{s}) \cdot \mathrm{g}^{*} \cdot \mathrm{d} \Omega
\end{aligned}
$$

$$
+\int_{\Omega} w_{\circ} \frac{\partial^{2} \mathrm{D}(\mathrm{s})}{\partial \mathrm{x}_{\mathrm{i}} \cdot \partial \mathrm{x}_{\mathrm{j}}} \cdot \mathrm{m}_{\mathrm{ij}}^{*} \mathrm{~d} \Omega+\int_{\Omega} w_{0} \frac{\partial \mathrm{D}(\mathrm{s})}{\partial \mathrm{x}_{\mathrm{j}}} \cdot \mathrm{q}_{\mathrm{j}}^{*} \cdot \mathrm{d} \Omega
$$

Logo, a partir de (3.29) e (3.36), tem-se:

$$
\frac{1}{\mathrm{D}_{0}}\left[\int_{\Gamma} \frac{\partial w}{\partial \mathrm{n}} \cdot \mathrm{D}(\mathrm{s}) \cdot \mathrm{M}_{\mathrm{n}}^{*} \cdot \mathrm{d} \Gamma-\sum_{\mathrm{i}=1}^{\mathrm{N}_{\mathrm{c}}} \mathbf{R}_{\mathrm{ci}}^{*} \cdot w_{\mathrm{ci}} \cdot \mathrm{D}_{\mathrm{ci}}-\int_{\Gamma} w \cdot \mathrm{D}(\mathrm{s}) \cdot \mathrm{q}_{\mathrm{n}}^{*} \cdot \mathrm{d} \Gamma-\int_{\Gamma} w \cdot \mathrm{D}(\mathrm{s}) \cdot \frac{\partial \mathbf{M}_{\mathrm{ns}}}{\partial \mathrm{s}} \cdot \mathrm{d} \Gamma\right.
$$




$$
\begin{aligned}
& -\int_{\Gamma} w \cdot \frac{\partial \mathrm{D}(\mathrm{s})}{\partial \mathrm{s}} \mathrm{M}_{\mathrm{ns}}^{*} \cdot \mathrm{d} \Gamma-\int_{\Gamma} w \cdot \frac{\partial \mathrm{D}(\mathrm{s})}{\partial \mathrm{x}_{\mathrm{j}}} \mathrm{m}_{\mathrm{ij}}^{*} \cdot \mathrm{n}_{\mathrm{i}} \cdot \mathrm{d} \Gamma+\int_{\Omega} w \cdot \frac{\partial \mathrm{D}(\mathrm{s})}{\partial \mathrm{x}_{\mathrm{i}}} \cdot \mathrm{q}_{\mathrm{i}}^{*} \cdot \mathrm{d} \Omega-\int_{\Omega} w \cdot \mathrm{D}(\mathrm{s}) \cdot \mathrm{g}^{*} \cdot \mathrm{d} \Omega \\
& \left.+\int_{\Omega} w \cdot \frac{\partial^{2} \mathrm{D}(\mathrm{s})}{\partial \mathrm{x}_{\mathrm{i}} \cdot \partial \mathrm{x}_{\mathrm{j}}} \cdot \mathrm{m}_{\mathrm{ij}}^{*} \cdot \mathrm{d} \Omega+\int_{\Omega} w \cdot \frac{\partial \mathrm{D}(\mathrm{s})}{\partial \mathrm{x}_{\mathrm{j}}} \cdot \mathrm{q}_{\mathrm{j}}^{*} \cdot \mathrm{d} \Omega\right] \\
& =\int_{\Gamma}\left(-V_{n} \cdot w^{*}+M_{n} \cdot \frac{\partial w^{*}}{\partial n}\right) \cdot d \Gamma-\sum_{i=1}^{N_{c}} R_{\mathrm{ci}} \cdot w_{\mathrm{ci}}^{*}-\int_{\Omega_{\mathrm{g}}} \mathrm{g} \cdot w^{*} \cdot \mathrm{d} \Omega_{\mathrm{g}}
\end{aligned}
$$

Conforme mencionado no item $3.2, \mathrm{~g}^{*}$ é uma carga unitária aplicada em um ponto " $q$ " qualquer do domínio da placa e sua representação matemática é a distribuição delta de Dirac $\delta(\mathbf{q}, \mathbf{p})$, e considerando-se a definição (2.62) tem-se que:

$$
\int_{\Omega} w(\mathbf{p}) \cdot \mathbf{D}(\mathrm{s}) \cdot \mathrm{g}^{\star} \cdot \mathrm{d} \Omega=\mathbf{D}(\mathbf{q}) \cdot w(\mathbf{q})
$$

Substituindo-se então $\mathbf{g}^{*}$ por $\delta(\boldsymbol{q}, \mathbf{p})$ em $(3.36)$ e usando-se a representação de cada variável em função dos dois pontos, "q" e "p", para os valores fundamentais, e em função de um ponto apenas em caso contrário, obtém-se:

$$
\begin{aligned}
& \frac{1}{\mathbf{D}_{0}}\left[\int_{\Gamma} \mathbf{D}(\mathbf{s}) \cdot\left[\frac{\partial w}{\partial \mathbf{n}}(\mathbf{P}) \cdot \mathbf{M}_{\mathbf{n}}^{*}(\mathbf{q}, \mathbf{P})-w(\mathbf{P}) \cdot \mathbf{V}_{\mathbf{n}}^{*}(\mathbf{q}, \mathbf{P})\right] \cdot \mathbf{d} \Gamma-\sum_{\mathbf{i}=1}^{N_{\mathrm{c}}} \mathbf{R}_{\mathrm{ci}}^{*}(\mathbf{q}, \mathbf{P}) \cdot w_{\mathrm{ci}}(\mathbf{P}) \cdot \mathbf{D}_{\mathrm{ci}}\right. \\
& -\int_{\Gamma}\left[\frac{\partial \mathbf{D}(\mathbf{s})}{\partial \mathbf{s}} \mathbf{M}_{\mathrm{ns}}^{*}(\mathbf{q}, \mathbf{P})+\frac{\partial \mathbf{D}(\mathbf{s})}{\partial \mathbf{x}_{\mathbf{j}}} \mathbf{m}_{\mathbf{i j}}^{*}(\mathbf{q}, \mathbf{P}) \cdot \mathbf{n}_{\mathbf{i}}\right] \cdot w(\mathbf{P}) \mathbf{d} \Gamma
\end{aligned}
$$




$$
\begin{aligned}
& \left.+\int_{\Omega}\left[2 \cdot \frac{\partial \mathbf{D}(\mathbf{s})}{\partial \mathbf{x}_{\mathbf{i}}} \cdot \mathbf{q}_{\mathrm{i}}^{*}(\mathbf{q}, \mathbf{P})+\frac{\partial^{2} \mathbf{D}(\mathbf{s})}{\partial \mathbf{x}_{\mathbf{i}} \cdot \partial \mathbf{x}_{\mathbf{j}}} \cdot \mathbf{m}_{\mathrm{ij}}^{*}(\mathbf{q}, \mathbf{P})\right] \cdot w(\mathbf{P}) \cdot \mathrm{d} \Omega-\mathbf{D}(\mathbf{q}) \cdot w(\mathbf{q})\right] \\
& =\int_{\Gamma}\left(-\mathbf{V}_{\mathrm{n}}(\mathbf{P}) \cdot w^{*}(\mathbf{q}, \mathbf{P})+\mathbf{M}_{\mathrm{n}}(\mathbf{P}) \cdot \frac{\partial w^{*}(\mathbf{q}, \mathbf{P})}{\partial \mathbf{n}}\right) \cdot \mathrm{d} \Gamma-\sum_{\mathrm{i}=1}^{N_{\mathrm{c}}} \mathbf{R}_{\mathrm{ci}}(\mathbf{P}) \cdot w_{\mathrm{ci}}^{*}(\mathbf{q}, \mathbf{P}) \\
& -\int_{\Omega_{\mathrm{g}}} \mathrm{g}(\mathrm{p}) \cdot w^{*}(\mathbf{q}, \mathbf{p}) \cdot \mathrm{d} \Omega_{\mathrm{g}}
\end{aligned}
$$

Reagrupando-se a eq.(3.39), obtém-se:

$$
\begin{aligned}
& \frac{\mathbf{D}(\mathbf{q})}{\mathbf{D}_{0}} \cdot w(\mathbf{q})+\frac{1}{\mathbf{D}_{0}} \int_{\Gamma} \cdot\left(\mathbf{D}(\mathbf{s}) \cdot \mathbf{V}_{\mathrm{n}}^{*}(\mathbf{q}, \mathbf{P})+\frac{\partial \mathbf{D}(\mathrm{s})}{\partial \mathrm{s}} \cdot \mathbf{M}_{\mathrm{ns}}^{*}+\frac{\partial \mathbf{D}(\mathbf{s})}{\partial \mathrm{x}_{\mathrm{j}}} \cdot \mathbf{m}_{\mathrm{ij}}^{*} \cdot \mathbf{n}_{\mathrm{i}}\right) \cdot w(\mathbf{P}) \cdot \mathrm{d} \Gamma \\
& -\frac{1}{\mathbf{D}_{0}} \int_{\Gamma} \mathrm{D}(\mathrm{s}) \cdot \frac{\partial w}{\partial \mathbf{n}}(\mathbf{P}) \cdot \mathbf{M}_{\mathrm{n}}^{*}(\mathbf{q}, \mathbf{P}) \cdot \mathbf{d} \Gamma+\sum_{\mathrm{i}=1}^{N_{\mathrm{c}}} \mathbf{R}_{\mathrm{ci}}^{*}(\mathbf{q}, \mathbf{P}) \cdot w_{\mathrm{ci}}(\mathbf{P}) \cdot \frac{\mathbf{D}_{\mathrm{ci}}}{\mathbf{D}_{\mathrm{o}}} \\
& -\frac{1}{D_{0}} \int_{\Omega}\left[2 \cdot \frac{\partial D(s)}{\partial x_{i}} \cdot q_{i}^{*}(\mathbf{q}, P)+\frac{\partial^{2} D(s)}{\partial x_{i} \cdot \partial x_{j}} \cdot m_{i j}^{*}(q, P)\right] \cdot w(p) \cdot d \Omega \\
& =\int_{\Gamma}\left(\mathbf{V}_{\mathrm{n}}(\mathbf{P}) \cdot w^{*}(\mathbf{q}, \mathbf{P})-\mathbf{M}_{\mathrm{n}}(\mathbf{P}) \cdot \frac{\partial w^{*}(\mathbf{q}, \mathbf{P})}{\partial \mathbf{n}}\right) \cdot \mathbf{d} \Gamma+\sum_{\mathrm{i}=1}^{N_{\mathrm{c}}} \mathbf{R}_{\mathrm{ci}}(\mathbf{P}) \cdot w_{\mathrm{ci}}^{*}(\mathbf{q}, \mathbf{P}) \\
& +\int_{\Omega_{g}} g(p) \cdot w^{n}(q, p) \cdot d \Omega_{g}
\end{aligned}
$$




\subsection{Equação Integral para um Ponto do Contorno da Placa}

As equações integrais de deslocamentos para placas com espessura constante, eq.(3.22), e com espessura variável, eq.(3.40), são válidas apenas para pontos contidos no interior do corpo em estudo. Deseja-se, por outro lado, obter a expressão correspondente para o caso em que o ponto de colocação $(\mathbf{q})$ pertença ao contorno $\Gamma$, uma vez que o método necessita desta equação para a montagem de um sistema de equações lineares.

O artificio utilizado comumente na literatura especializada, DOMINGUEZ \& BREBBIA (1989), para a avaliação de pontos pertencentes ao contorno(Q), consiste transformar o ponto do contorno num ponto pertencente ao domínio, acrescentandose, para isto, parte de um círculo de raio $\varepsilon$, denominado $\Omega_{\xi}$, centrado no ponto de colocação em questão. Assim, um ponto $\mathbf{Q}$ do contorno, figura 3.5, passa a ser um ponto q do domínio, valendo então, para este ponto, as equações (3.22) e (3.40).

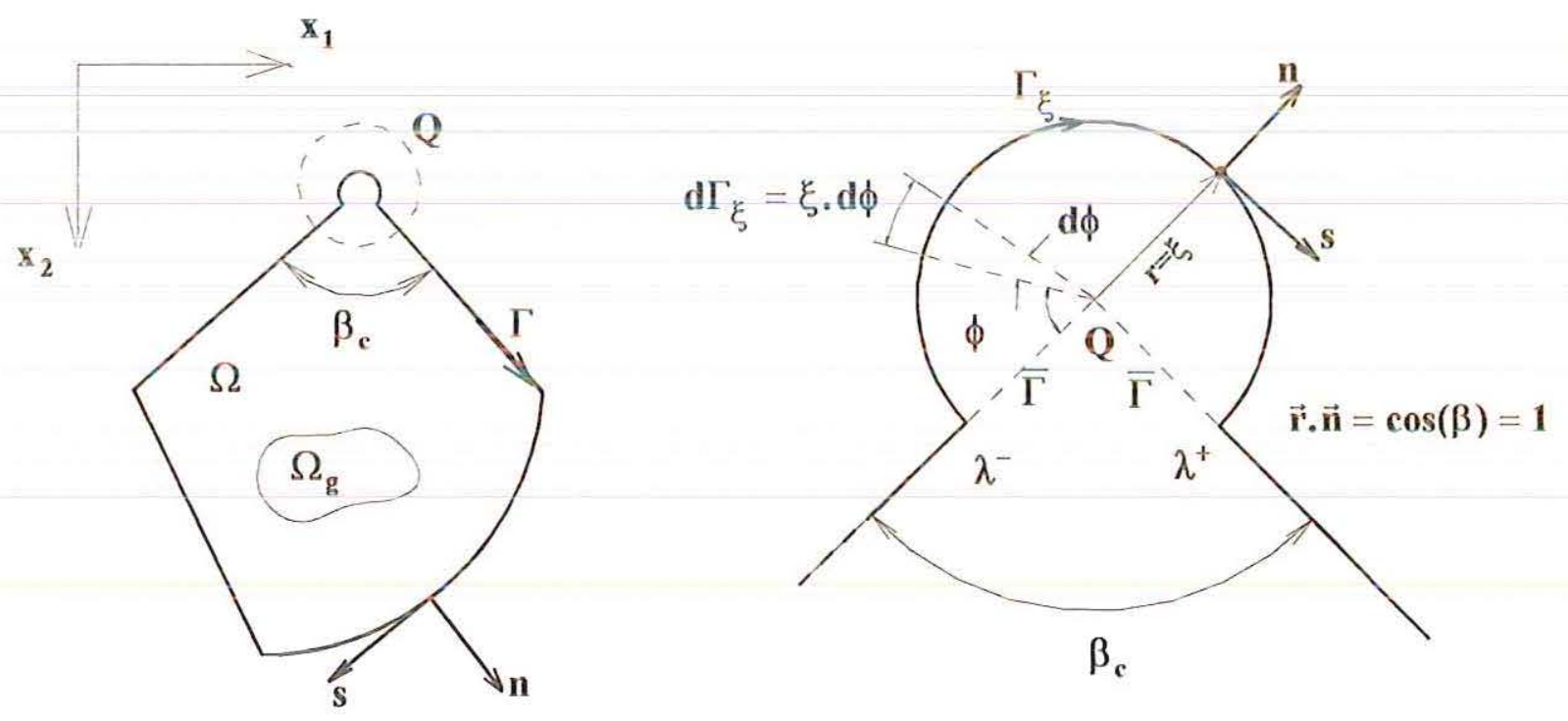

FIGURA 3.5 - Contorno Circular Acrescentado a um Ponto Q . 
Com esta modificação, o novo domínio torna-se $\Omega+\Omega_{\xi}$. O contorno passa então a ser $\Gamma-\bar{\Gamma}+\Gamma_{\xi}$. Considerando-se tal artificio na eq.(3.22), esta passa a se apresentar da seguinte forma:

$$
\begin{aligned}
& w(\mathbf{Q})+\int_{\Gamma-\bar{\Gamma}}\left[\mathbf{V}_{\mathrm{n}}^{*}(\mathbf{Q}, \mathbf{P}) \cdot w(\mathbf{P})-\mathbf{M}_{\mathrm{n}}^{*}(\mathbf{Q}, \mathbf{P}) \cdot \frac{\partial w}{\partial \mathrm{n}}(\mathbf{P})\right] \cdot \mathrm{d} \Gamma(\mathbf{P}) \\
& +\int\left[V_{n}^{*}(\mathbf{Q}, \mathbf{P}) \cdot w(\mathbf{P})-\mathbf{M}_{n}^{*}(\mathbf{Q}, \mathbf{P}) \cdot \frac{\partial w}{\partial \mathbf{n}}(\mathbf{P})\right] \cdot \mathrm{d} \Gamma(\mathbf{P})+\sum_{\mathrm{i}=1}^{N_{\mathrm{c}}-1} \mathbf{R}_{\mathrm{ci}}^{*}(\mathbf{Q}, \mathbf{P}) \cdot w_{\mathrm{ci}}(\mathbf{P}) \\
& \Gamma_{\xi} \\
& +\mathbf{R}_{\lambda^{-}}^{*}(\mathbf{Q}, \mathbf{P}) \cdot w_{c^{-}}(\mathbf{P})+\mathbf{R}_{\lambda^{+}}^{*}(\mathbf{Q}, \mathbf{P}) \cdot w_{\mathbf{c}^{+}}(\mathbf{P}) \\
& =\int_{\Gamma-\Gamma}\left[V_{n}(\mathbf{P}) \cdot w^{*}(\mathbf{Q}, \mathbf{P})-\mathbf{M}_{\mathbf{n}}(\mathbf{P}) \cdot \frac{\partial w^{*}}{\partial \mathbf{n}}(\mathbf{Q}, \mathbf{P})\right] \cdot \mathrm{d} \Gamma(\mathbf{P}) \\
& +\int\left[V_{\mathbf{n}}(\mathbf{P}) \cdot w^{*}(\mathbf{Q}, \mathbf{P})-\mathbf{M}_{\mathbf{n}}(\mathbf{P}) \cdot \frac{\partial w^{*}}{\partial \mathbf{n}}(\mathbf{Q}, \mathbf{P})\right] \cdot \mathbf{d} \Gamma_{\xi}(\mathbb{P}) \\
& \Gamma_{\xi}
\end{aligned}
$$

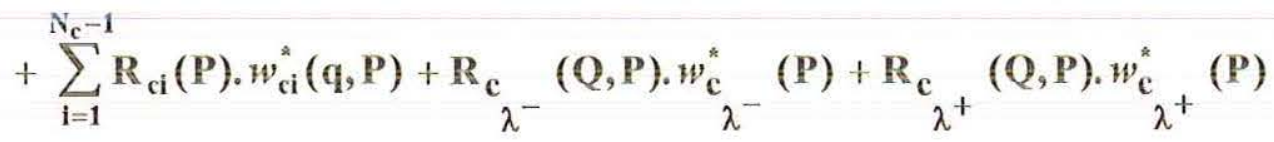

$$
\begin{aligned}
& +\int_{\Omega_{g}} g(p) \cdot w^{*}(Q, p) \cdot d \Omega_{g}(p)
\end{aligned}
$$

Assim, o ponto $\mathbf{Q}$ será do contorno quando o raio $\xi$ tender a zero e $w(\mathbf{Q})$ será calculado na condição limite. Portanto: 


$$
\begin{aligned}
& w(\mathbf{Q})+\lim _{\bar{\Gamma} \rightarrow 0} \int_{\Gamma-\bar{\Gamma}}\left[V_{n}^{*}(\mathbf{Q}, \mathbf{P}) \cdot w(\mathbf{P})-\mathbf{M}_{\mathrm{n}}^{*}(\mathbf{Q}, \mathbf{P}) \cdot \frac{\partial w}{\partial \mathrm{n}}(\mathbf{P})\right] \cdot \mathrm{d} \Gamma(\mathrm{P}) \\
& +\lim _{\xi \rightarrow 0} \int_{\Gamma_{\xi}}\left[\mathbf{V}_{\mathbf{n}}^{*}(\mathbf{Q}, \mathbf{P}) \cdot w(\mathbf{P})-\mathbf{M}_{\mathbf{n}}^{*}(\mathbf{Q}, \mathbf{P}) \cdot \frac{\partial w}{\partial \mathbf{n}}(\mathbf{P})\right] \cdot \mathrm{d} \Gamma(\mathbf{P})+\sum_{\mathrm{i}=1}^{N_{\mathrm{c}}-\mathbf{1}} \mathbf{R}_{\mathrm{ci}}^{*}(\mathbf{Q}, \mathbf{P}) \cdot w_{\mathrm{ci}}(\mathbf{P}) \\
& +\lim _{\xi \rightarrow 0}\left[\mathbf{R}_{\lambda^{-}}^{*}(\mathbf{Q}, \mathbf{P}) \cdot w_{\mathbf{c}^{-}}(\mathbf{P})+\mathbf{R}_{\mathbf{c}^{+}}^{*}(\mathbf{Q}, \mathbf{P}) \cdot w_{\lambda^{+}}(\mathbf{P})\right] \\
& =\lim _{\bar{\Gamma}>0} \int_{\Gamma-\bar{\Gamma}}\left[V_{n}(P) \cdot w^{*}(\mathbf{Q}, \mathbf{P})-M_{n}(P) \cdot \frac{\partial w^{*}}{\partial n}(\mathbf{Q}, \mathbf{P})\right] \cdot \mathrm{d} \Gamma(\mathbf{P}) \\
& +\lim _{\xi \rightarrow 0} \int_{\Gamma_{\xi}}\left[V_{n}(P) \cdot w^{*}(Q, P)-M_{n}(P) \cdot \frac{\partial w^{*}}{\partial n}(\mathbf{Q}, P)\right] \cdot d \Gamma_{\xi}(P) \\
& +\sum_{i=1}^{N_{c}-1} R_{c i}(P), w_{c i}^{*}(\mathbf{Q}, \mathbf{P})+\lim _{\zeta \rightarrow 0}\left[\mathbf{R}_{c_{\lambda^{-}}}(\mathbf{Q}, \mathbf{P}) \cdot w_{c^{-}}^{*}(\mathbf{P})+\mathbf{R}_{c^{+}}(\mathbf{Q}, \mathbf{P}), w_{\lambda^{+}}^{*}(\mathbf{P})\right] \\
& +\int_{\Omega_{g}} g(p), w^{*}(Q, p) \cdot d \Omega_{g}(p)
\end{aligned}
$$

Os limites das integrais sobre $(\Gamma-\bar{\Gamma})$ indicadas em (3.42), por definição, representam o valor principal das mesmas. Assim:

$$
\begin{aligned}
\lim _{\bar{\Gamma} \rightarrow \mathbf{0}} \int_{\Gamma-\bar{\Gamma}}\left[V_{n}^{*}(\mathbf{Q}, \mathbf{P}) \cdot w(\mathbf{P})-\mathbf{M}_{\mathbf{n}}^{*}(\mathbf{Q}, \mathbf{P}) \cdot \frac{\partial w}{\partial \mathbf{n}}(\mathbf{P})\right] \cdot \mathrm{d} \Gamma(\mathbf{P}) \\
=\int_{\Gamma}\left[\mathbf{V}_{\mathbf{n}}^{*}(\mathbf{Q}, \mathbf{P}) \cdot w(\mathbf{P})-\mathbf{M}_{\mathbf{n}}^{*}(\mathbf{Q}, \mathbf{P}) \cdot \frac{\partial w}{\partial \mathbf{n}}(\mathbf{P})\right] \cdot \mathbf{d} \Gamma(\mathbf{P})
\end{aligned}
$$


$\lim _{\bar{\Gamma} \rightarrow 0} \int_{\Gamma-\bar{\Gamma}}\left[V_{n}(P) \cdot w^{*}(Q, P)-M_{n}(P) \cdot \frac{\partial w^{*}}{\partial n}(Q, P)\right] \cdot d \Gamma(P)$

$$
=\int_{\Gamma}\left[V_{n}(\mathbf{P}) \cdot w^{*}(\mathbf{Q}, \mathbf{P})-\mathbf{M}_{\mathbf{n}}(\mathbf{P}) \cdot \frac{\partial w^{*}}{\partial \mathrm{n}}(\mathbf{Q}, \mathbf{P})\right] \cdot \mathrm{d} \Gamma(\mathbf{P})
$$

(3.44)

As parcelas referentes ao canto, resultam:

$$
\begin{aligned}
\left(\sum_{\mathbf{i}=1}^{N_{\mathrm{c}}-1} \mathbf{R}_{\mathrm{ci}}^{*}(\mathbf{Q}, \mathbf{P}) \cdot w_{\mathrm{ci}}(\mathbf{P})+\lim _{\xi \rightarrow 0}\right. & {\left.\left[\mathbf{R}_{\lambda^{-}}^{*}(\mathbf{Q}, \mathbf{P}) \cdot w_{\mathbf{c}^{-}}(\mathbf{P})+\mathbf{R}_{\lambda^{+}}^{*}(\mathbf{Q}, \mathbf{P}) \cdot w_{\mathbf{c}_{\lambda^{+}}}(\mathbf{P})\right]\right) } \\
& =\sum_{\mathrm{i}=1}^{N_{\mathrm{c}}} \mathbf{R}_{\mathrm{ci}}^{*}(\mathbf{Q}, \mathbf{P}) \cdot w_{\mathrm{ci}}(\mathbf{P})
\end{aligned}
$$

$$
\begin{aligned}
& \left(\sum_{\mathrm{i}=1}^{N_{\mathrm{c}}-1} \mathbb{R}_{\mathrm{ci}}(\mathrm{Q}, \mathrm{P}) \cdot w_{\mathrm{ci}}^{*}(\mathrm{P})+\lim _{\xi \rightarrow 0}\left[\mathbb{R}_{\lambda^{-}}(\mathrm{Q}, \mathrm{P}) \cdot w_{\mathrm{c}^{*}}{ }_{\lambda^{-}}(\mathrm{P})+\mathbb{R}_{\lambda^{+}}(\mathrm{Q}, \mathrm{P}) \cdot w_{\lambda^{+}}{ }_{\mathrm{k}}{ }^{*}(\mathrm{P})\right]\right) \\
& =\sum_{i=1}^{N_{c}} R_{c i}(Q, P), w_{c i}^{k}(P)
\end{aligned}
$$

A parcela de (3.42) referente à integral sobre o trecho $\Gamma_{\xi}$, envolvendo $w(\mathbb{P}) \mathrm{e}$ $\partial w(\mathbf{P}) / \partial \mathbf{n}$, pode ser rescrita da seguinte forma:

$$
\begin{aligned}
& \lim _{\xi \rightarrow \mathbf{0}} \int\left[\mathbf{V}_{\mathbf{n}}^{*}(\mathbf{Q}, \mathbf{P}) \cdot w(\mathbf{P})-\mathbf{M}_{\mathbf{n}}^{*}(\mathbf{Q}, \mathbf{P}) \cdot \frac{\partial w}{\partial \mathbf{n}}(\mathbf{P})\right] \cdot \mathbf{d} \Gamma(\mathbf{P}) \\
& =\lim _{\xi \rightarrow \mathbf{0}} \int\left\{\mathbf{V}_{\mathbf{n}}^{*}(\mathbf{Q}, \mathbf{P}) \cdot[w(\mathbf{P})-w(\mathbf{Q})]-\mathbf{M}_{\mathbf{n}}^{*}(\mathbf{Q}, \mathbf{P}) \cdot\left[\frac{\partial w}{\partial \mathbf{n}}(\mathbf{P})-\frac{\partial w}{\partial \mathbf{n}}(\mathbf{Q})\right]\right\} \cdot \mathrm{d} \Gamma_{\xi}(\mathbf{P})
\end{aligned}
$$




$$
+\lim _{\xi \rightarrow 0} \int_{\Gamma_{\xi}} V_{n}^{*}(Q, P) \cdot w(Q) \cdot d \Gamma_{\xi}(P)-\lim _{\xi \rightarrow 0} \int_{\Gamma_{\xi}} M_{n}^{*}(Q, P) \cdot \frac{\partial w}{\partial n}(Q) \cdot d \Gamma_{\xi}(P)
$$

Considerando-se válida a continuidade ou utilizando-se a condição de Hölder, JASWON \& SYMM (1977), dada por:

$$
\begin{aligned}
& |w(\mathbf{P})-w(\mathbf{Q})| \leq \mathrm{C}_{1} \cdot \mathrm{r}^{\alpha_{1}}(\mathrm{P}, \mathrm{Q}) \\
& \left|\frac{\partial w}{\partial \mathrm{n}}(\mathrm{P})-\frac{\partial w}{\partial \mathrm{n}}(\mathrm{Q})\right| \leq \mathrm{C}_{2} \cdot \mathrm{r}^{\alpha}(\mathrm{P}, \mathrm{Q})
\end{aligned}
$$

onde $\mathbf{C}_{1}$ e $\mathbf{C}_{2}$ são constantes e $\mathbf{0}<\alpha_{\mathbf{i}} \leq \mathbf{1}$, com $(\mathbf{i}=\mathbf{1}, 2)$, a primeira integral de (3.47) se anula. Como $w(\mathbf{P})$ e $\partial w(\mathbf{P}) / \partial \mathbf{n}$ são valores do domínio e não variam, portanto, ao longo de $\Gamma_{\xi}$, a eq.(3.47) fica:

$$
\begin{aligned}
& \lim _{\xi \rightarrow 0} \int\left[V_{\Gamma_{\xi}}^{*}(\mathbf{Q}, \mathbf{P}) \cdot w(\mathbf{Q})-\mathbf{M}_{\mathrm{n}}^{\mathrm{*}}(\mathbf{Q}, \mathbf{P}) \cdot \frac{\partial w}{\partial \mathrm{n}}(\mathbf{Q})\right] \cdot \mathrm{d} \Gamma_{\xi}(\mathbf{P}) \\
& =w(\mathrm{Q}) \cdot \lim _{\xi \rightarrow 0} \int_{\Gamma_{\xi}} V_{\mathrm{n}}^{*}(\mathrm{Q}, \mathrm{P}) \cdot \mathrm{d} \Gamma_{\zeta}(\mathrm{P})-\frac{\partial w}{\partial \mathrm{n}}(\mathrm{Q}) \cdot \lim _{\xi \rightarrow 0} \int_{\Gamma_{\xi}} \mathrm{M}_{\mathrm{n}}^{*}(\mathrm{Q}, \mathrm{P}) \cdot \mathrm{d} \Gamma_{\xi}(\mathrm{P})
\end{aligned}
$$

Substituindo-se em (3.48) os valores de $\mathbf{V}_{\mathbf{n}}^{*}(\mathbf{Q}, \mathbf{P})$ e $\mathbf{M}_{\mathbf{n}}^{*}(\mathbf{Q}, \mathbf{P})$, dados em (2.99) e (2.97), respectivamente, e considerando-se ainda, que, no caso, $\mathbf{r}_{\mathbf{9}_{\mathbf{i}}} \cdot \mathbf{n}_{\mathbf{i}}=\mathbf{1}$, $\boldsymbol{r}_{\mathbf{i}_{\mathbf{i}}} \cdot \mathrm{s}_{\mathbf{s}_{\mathbf{i}}}=\mathbf{0}$ e $\mathbf{r}=\mathbf{R}=\xi$, figura 3.5 , obtém-se:

$$
\lim _{\xi \rightarrow 0} \int_{\Gamma_{\xi}}\left[V_{n}^{*}(\mathbf{Q}, \mathbf{P}) \cdot w(\mathbf{Q})-\mathbf{M}_{\mathbf{n}}^{*}(\mathbf{Q}, \mathbf{P}) \cdot \frac{\partial w}{\partial \mathbf{n}}(\mathbf{Q})\right] \cdot \mathrm{d} \Gamma_{\xi}(\mathbf{P})
$$




$$
=w(Q) \cdot \lim _{\xi \rightarrow 0} \int_{\Gamma_{\xi}} \frac{-1}{2 . \pi \cdot \xi} \cdot \mathrm{d} \Gamma_{\xi}(P)-\frac{\partial w}{\partial \mathrm{n}}(\mathrm{Q}) \cdot \lim _{\xi \rightarrow 0} \int_{\Gamma_{\xi}} \frac{1}{4 \cdot \pi}[(1+v) \cdot \ln \xi+1] \cdot \mathrm{d} \Gamma_{\xi}(\mathrm{P})
$$

Sendo $\mathbf{d} \Gamma_{\xi}(\mathbf{P})=\xi$.d $\phi$, a expressão (3.51) pode ser indicada na forma:

$$
\begin{aligned}
& \lim _{\xi \rightarrow 0} \int_{\Gamma_{\xi}}\left[V_{n}^{*}(\mathbf{Q}, \mathbf{P}) \cdot w(\mathbf{Q})-\mathbf{M}_{\mathrm{n}}^{*}(\mathbf{Q}, \mathbf{P}) \cdot \frac{\partial w}{\partial \mathrm{n}}(\mathbf{Q})\right] \cdot \mathrm{d} \Gamma_{\xi}(\mathbf{P}) \\
& =w(Q) \cdot \lim _{\xi \rightarrow 0} \int_{0}^{2 . \pi-\beta_{c}} \frac{-1}{2 \cdot \pi \cdot \xi} \cdot \xi \cdot \hat{d} \psi+\frac{\partial w}{\partial n}(Q) \cdot \lim _{\xi \rightarrow 0} \int_{0}^{2 . \pi-\beta_{c}} \frac{1}{4 \cdot \pi}[(1+v) \cdot \ln \xi+1] \cdot \xi \cdot d \hat{d}
\end{aligned}
$$

onde $\beta_{c}$ é o ângulo interno do canto da placa, indicado na figura 3.5. Desenvolvendo-se as integrais de (3.52), obtém-se:

$$
\lim _{\xi \rightarrow 0} \int\left[V_{\Gamma_{\xi}}^{*}(\mathbf{Q}, \mathbf{P}) \cdot w(\mathbf{Q})-\mathbf{M}_{\mathbf{n}}^{*}(\mathbf{Q}, \mathbf{P}) \cdot \frac{\partial w}{\partial \mathbf{n}}(\mathbf{Q})\right] \cdot \mathrm{d} \Gamma_{\xi}(\mathbf{P})=-\frac{2 \cdot \pi-\beta_{\mathrm{c}}}{2 \cdot \pi} \cdot w(\mathbf{Q})
$$

Analogamente, as demais integrais sobre $\Gamma_{\xi}$ indicadas em (3.42) conduzem a valores nulos. Assim, a eq.(3.42) pode ser rescrita como:

$$
\begin{aligned}
& \mathrm{C}(\mathrm{Q}) \cdot w(\mathrm{Q})+\int_{\Gamma}\left[\mathrm{V}_{\mathrm{n}}^{*}(\mathrm{Q}, \mathrm{P}) \cdot w(\mathrm{P})-\mathrm{M}_{\mathrm{n}}^{\mathrm{*}}(\mathrm{Q}, \mathrm{P}) \cdot \frac{\partial w}{\partial \mathrm{n}}(\mathrm{P})\right] \cdot \mathrm{d} \Gamma(\mathrm{P}) \\
& +\sum_{\mathrm{i}=1}^{N_{\mathrm{c}}} \mathbf{R}_{\mathrm{ci}}^{*}(\mathbf{Q}, \mathbf{P}) \cdot w_{\mathrm{ci}}(\mathbf{P})=\int_{\Gamma}\left[\mathrm{V}_{\mathrm{n}}(\mathbf{P}) \cdot w^{*}(\mathbf{Q}, \mathbf{P})-\mathbf{M}_{\mathrm{n}}(\mathbf{P}) \cdot \frac{\partial w^{*}}{\partial \mathrm{n}}(\mathbf{Q}, \mathbf{P})\right] \cdot \mathrm{d} \Gamma(\mathbf{P})
\end{aligned}
$$






$$
+\sum_{\mathrm{i}=1}^{\mathrm{N}_{\mathrm{c}}} \mathbf{R}_{\mathrm{ci}}(\mathbf{P}) \cdot w_{\mathrm{ci}}^{*}(\mathbf{Q}, \mathbf{P})+\int_{\Omega_{\mathrm{g}}} \mathrm{g}(\mathbf{p}) \cdot w^{*}(\mathbf{Q}, \mathbf{p}) \cdot \mathrm{d} \Omega_{\mathrm{g}}(\mathbf{p})
$$

onde:

$$
\mathbf{C}(\mathbf{Q})=\frac{\beta_{\mathbf{c}}}{2 . \pi}
$$

Para os pontos "A" fora do domínio, uma vez que o primeiro termo da equação (3.54) se anula, devido à propriedade da função delta de Dirac, ou seja:

$$
\int \delta(\mathbf{A}, \mathbf{P}) \cdot w(\mathbf{p}) \cdot \mathbf{d} \Omega(\mathbf{p})=w(\hat{A})=0
$$

Com isso, pode-se concluir que:

$$
C(Q)= \begin{cases}1, & Q \in \Omega \text { (domínio) } \\ \frac{\beta_{c}}{2 \cdot \pi}, & Q \in \Gamma \text { (contorno) } \\ 0, & Q \notin \Omega \cap Q \notin \Gamma(\text { externo) }\end{cases}
$$

Para ponto do contorno onde o elemento é linear, tem-se que $\beta_{\mathrm{c}}=\pi$, logo:

$$
\mathrm{C}(\mathrm{Q})=0,5
$$

De forma análoga ao efetuado no item 3.4 , pode-se obter a equação integral de rotação $\partial w(\mathbf{Q}) / \partial \mathbf{m}$, eq.(3.23), para um ponto do contorno, em relação a uma coordenada genérica $\mathrm{m}$. Como neste trabalho será utilizado uma forma alternativa, utilizando-se apenas as equações integrais de deslocamentos, não haverá necessidade da formulação da equação integral de rotação no contorno, podendo esta dedução ser claramente encontrada em PAIVA (1987). 
Seguindo-se o mesmo procedimento a equação integral do deslocamento transversal para a placa com espessura variável (3.39) ou (3.40) pode ser escrita como:

$$
\begin{aligned}
& \mathrm{C}(\mathrm{Q}) \cdot \frac{\mathrm{D}(\mathrm{Q})}{\mathrm{D}_{\mathrm{o}}} \cdot w(\mathrm{Q})+\frac{1}{\mathrm{D}_{0}} \int_{\Gamma} \cdot\left(\mathrm{D}(\mathrm{s}) \cdot \mathrm{V}_{\mathrm{n}}^{*}(\mathrm{Q}, \mathrm{P})+\frac{\partial \mathrm{D}(\mathrm{s})}{\partial \mathrm{s}} \cdot \mathrm{M}_{\mathrm{ns}}^{*}+\frac{\partial \mathrm{D}(\mathrm{s})}{\partial \mathrm{x}_{\mathrm{j}}} \cdot \mathrm{m}_{\mathrm{ij}}^{*} \cdot \mathrm{n}_{\mathrm{i}}\right) \cdot w(\mathrm{P}) \cdot \mathrm{d} \Gamma \\
& -\frac{1}{\mathbf{D}_{0}} \int_{\Gamma} \mathbf{D}(\mathrm{s}) \cdot \frac{\partial w}{\partial \mathbf{n}}(\mathbf{P}) \cdot \mathbf{M}_{\mathrm{n}}^{*}(\mathbf{Q}, \mathbf{P}) \cdot \mathbf{d} \Gamma+\sum_{\mathrm{i}=1}^{\mathrm{N}_{\mathrm{c}}} \mathbf{R}_{\mathrm{ci}}^{*}(\mathbf{Q}, \mathbf{P}) \cdot w_{\mathrm{ci}}(\mathbf{P}) \cdot \frac{\mathbf{D}_{\mathrm{ci}}}{\mathbf{D}_{\mathrm{o}}} \\
& -\frac{1}{D_{0}} \int_{\Omega}\left[2 \cdot \frac{\partial \mathrm{D}(\mathrm{s})}{\partial \mathrm{x}_{\mathrm{i}}} \cdot \mathrm{q}_{\mathrm{i}}^{\mathrm{*}}(\mathrm{Q}, \mathrm{P})+\frac{\partial^{2} \mathrm{D}(\mathrm{s})}{\partial \mathrm{x}_{\mathrm{i}} \cdot \partial \mathrm{x}_{\mathrm{j}}} \cdot \mathrm{m}_{\mathrm{ij}}^{\mathrm{k}}(\mathrm{Q}, \mathrm{P})\right] \cdot w(\mathrm{p}) \cdot \mathrm{d} \Omega \\
& =\int_{\Gamma}\left(\mathbf{V}_{\mathrm{n}}(\mathbf{P}) \cdot w^{*}(\mathbf{q}, \mathbf{P})-\mathbf{M}_{\mathrm{n}}(\mathbf{P}) \cdot \frac{\partial w^{*}(\mathrm{Q}, \mathrm{P})}{\partial \mathrm{n}}\right) \cdot \mathrm{d} \Gamma+\sum_{\mathrm{i}=1}^{\mathrm{N}_{\mathrm{c}}} \mathbf{R}_{\mathrm{ci}}(\mathrm{P}) \cdot w_{\mathrm{ci}}^{*}(\mathrm{Q}, \mathrm{P})
\end{aligned}
$$$$
+\int_{\Omega_{g}} g(p) \cdot w^{\alpha}(Q, p) \cdot d \Omega g
$$

A parcela da integral de contorno da eq.(3.58):

$$
\frac{\partial \mathbf{D}(\mathbf{s})}{\partial \mathbf{x}_{\mathbf{j}}} \cdot \mathbf{m}_{\mathbf{i j}}^{*} \cdot \mathbf{n}_{\mathbf{i}}
$$

pode ser escrita como: 


$$
\frac{\partial \mathbf{D}(\mathbf{s})}{\partial \mathbf{x}_{\mathbf{j}}} \cdot \mathbf{M}_{\mathbf{j}}^{*}
$$

Escrevendo-se (3.60) na sua forma explícita, resulta:

$$
\frac{\partial \mathbf{D}(\mathbf{s})}{\partial \mathbf{x}_{\mathbf{j}}} \cdot \mathbf{M}_{\mathbf{j}}^{*}=\left\{\begin{array}{l}
\left(\frac{\partial \mathbf{D}}{\partial \mathbf{n}} \cdot \mathbf{n}_{1}+\frac{\partial \mathbf{D}}{\partial \mathrm{s}} \cdot \mathbf{s}_{1}\right) \\
\left(\frac{\partial \mathbf{D}}{\partial \mathbf{n}} \cdot \mathbf{n}_{2}+\frac{\partial \mathbf{D}}{\partial \mathrm{s}} \cdot \mathbf{s}_{2}\right)
\end{array}\right\} \cdot\left\{\begin{array}{l}
\mathbf{M}_{1}^{*} \\
\mathbf{M}_{2}^{*}
\end{array}\right\}
$$

Utilizando-se da transformação de coordenadas dada em (2.32.a) para expressar M nas coordenadas n e s, pode-se escrever (3.61) como:

$$
\frac{\partial D(s)}{\partial x_{j}} \cdot M_{j}^{*}=\left[\begin{array}{ll}
\frac{\partial D}{\partial n} & \frac{\partial D}{\partial s}
\end{array}\right] \cdot\left[\begin{array}{cc}
n_{1} & n_{2} \\
s_{1} & s_{2}
\end{array}\right] \cdot\left[\begin{array}{ll}
n_{1} & s_{1} \\
n_{2} & s_{2}
\end{array}\right] \cdot\left\{\begin{array}{l}
M_{n}^{*} \\
M_{n s}^{*}
\end{array}\right\}
$$

Fazendo-se as operações de matrizes, obtém-se:

$$
\frac{\partial \mathbf{D}(s)}{\partial \mathbf{x}_{\mathbf{j}}} \cdot \mathbf{M}_{\mathbf{j}}^{*}=\frac{\partial \mathbf{D}}{\partial \mathbf{n}} \cdot \mathbf{M}_{n}^{*}+\frac{\partial \mathbf{D}}{\partial s} \cdot \mathbf{M}_{n s}^{*}
$$

Substituindo-se (3.63) em (3.58), obtém-se:

$$
\begin{aligned}
& C(Q) \cdot \frac{D(Q)}{D_{0}} \cdot w(Q)+\frac{1}{D_{0}} \int_{\Gamma} \cdot\left(D(s) \cdot V_{n}^{*}(Q, P)+2 \cdot \frac{\partial D(s)}{\partial s} \cdot M_{n s}^{*}+\frac{\partial D(s)}{\partial n} \cdot M_{n}^{*}\right) \cdot w(P) \cdot d \Gamma \\
& -\frac{1}{D_{0}} \int_{\Gamma} D(s) \cdot \frac{\partial w}{\partial n}(\mathbb{P}) \cdot M_{n}^{*}(Q, P) \cdot d \Gamma+\sum_{i=1}^{N_{c}} R_{c i}^{*}(Q, P) \cdot w_{c i}(P) \cdot \frac{D_{c i}}{D_{0}}
\end{aligned}
$$




$$
\begin{aligned}
& -\frac{1}{D_{0}} \int_{\Omega}\left[2 \cdot \frac{\partial D(s)}{\partial x_{i}} \cdot q_{i}^{*}(Q, P)+\frac{\partial^{2} D(s)}{\partial x_{i} \cdot \partial x_{j}} \cdot m_{i j}^{*}(Q, P)\right] \cdot w(p) \cdot d \Omega \\
& =\int_{\Gamma}\left(\mathbf{V}_{\mathrm{n}}(\mathbf{P}) \cdot w^{*}(\mathbf{q}, \mathbf{P})-\mathbf{M}_{\mathrm{n}}(\mathbf{P}) \cdot \frac{\partial w^{*}(\mathbf{Q}, \mathbf{P})}{\partial \mathrm{n}}\right) \cdot \mathrm{d} \Gamma+\sum_{\mathrm{i}=1}^{N_{\mathrm{c}}} \mathbf{R}_{\mathrm{ci}}(\mathbf{P}) \cdot w_{\mathrm{ci}}^{*}(\mathbf{Q}, \mathbf{P}) \\
& +\int_{\Omega_{g}} g(p) \cdot w^{*}(Q, p) \cdot d \Omega_{g}
\end{aligned}
$$




\section{EQUAÇÕES ALGÉBRICAS DO MEC PARA PLACA DE KIRCHHOFF}

\subsection{Introdução}

Neste capítulo será apresentada a formulação numérica para resolução aproximada das equações integrais de placas vistas anteriormente.

As equações integrais do deslocamento e de sua derivada direcional, obtidas no capítulo anterior, são praticamente impossiveis de serem resolvidas analiticamente na maioria dos casos de interesse estrutural prático. Tem-se então a necessidade da aplicação de métodos numéricos, transformando as equações integrais em equações algébricas envolvendo os valores nodais das variáveis do contorno, que são os valores das variáveis associadas aos nós, obtendo-se, assim, soluções aproximadas. Essa transformação é feita discretizando-se o contorno da placa em segmentos chamados "elementos de contorno", onde os deslocamentos e esforços são aproximados por funções previamente escolhidas. As integrais de domínio que aparecem na equação integral de deslocamento serão transformadas em integrais de contorno e estas também serão resolvidas numericamente.

Neste capítulo mostra-se a transformação de uma equação integral em equação algébrica. Como conseqüência pode-se também gerar o sistema de equações lineares que governa o problema de placas.

Para o caso de placas com espessura variável, aparece um termo de domínio que acarretará em pontos internos como incógnita de deslocamentos. 


\subsection{Discretização do Contorno}

Considere-se a figura 4.1, onde o contorno da placa está aproximado por um número finito de segmentos ou "elementos de contorno" $\Gamma_{\mathrm{j}}$. $\mathrm{O}$ número e a forma dos elementos são escolhidos de modo que o contorno real fique representado exato ou aproximadamente. Para isto, pode ser adotado elemento de geometria reta ou curva.

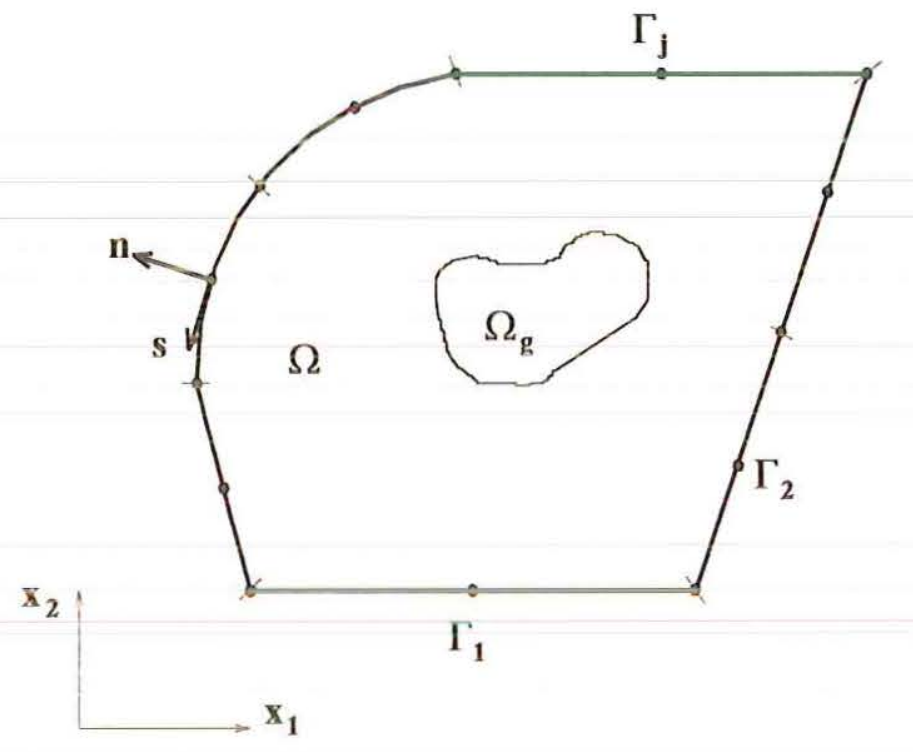

FIGURA 4.1 - Discretização do Contorno da Placa.

Quanto a geometria, os elementos de contorno mais usados podem ser, figura 4.2:

- retos - cada elemento é definido por dois pontos nodais;

- curvos - $\left\{\begin{array}{l}\text { quadrático, definido por três pontos nodais; } \\ \text { circular, definido por três pontos nodais; } \\ \text { cúbico, definido por quatro pontos nodais. }\end{array}\right.$ 


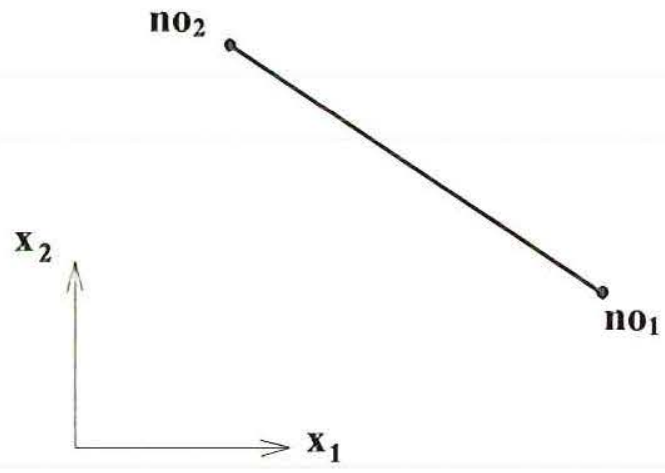

(a) Elemento Reto

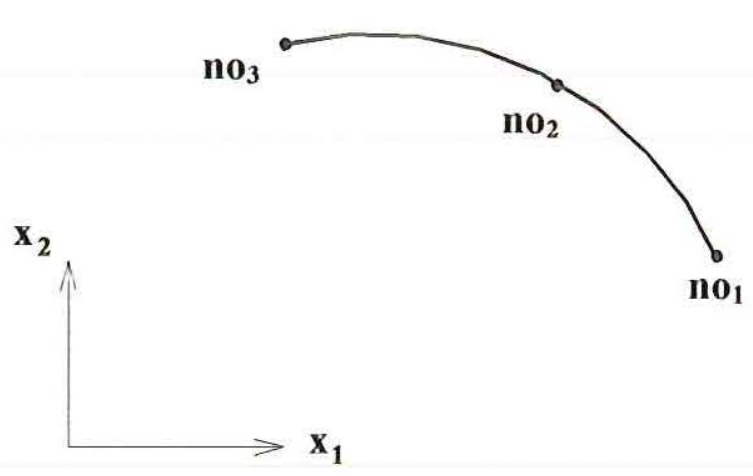

(b) Elemento Curvo

FIGURA 4.2 - Geometria dos Elementos de Contorno.

Neste trabalho, adotam-se segmentos retos, ou seja, elementos lineares quanto à geometria, figura 4.2a.

Como será visto mais adiante, as integrais sobre esses elementos, quando não singulares, são feitas numericamente. Assim é conveniente expressar as coordenadas de cada ponto "P" de um elemento em função de coordenadas locais homogêneas $\xi$, figura 4.3, da seguinte forma:

$$
\underset{\sim}{\mathrm{x}}(\mathbf{P})=\underset{\sim}{\underset{\sim}{\mathrm{T}}}(\mathbf{P}) \cdot \underset{\sim}{\mathbb{X}^{N}}
$$

onde:

$$
\begin{aligned}
& \underset{\sim}{x}(P)=\left\{\begin{array}{l}
x_{1}(P) \\
x_{2}(P)
\end{array}\right\} \\
& \underset{\sim}{\Psi^{\mathrm{T}}}(\mathbf{P})=\left[\begin{array}{cc}
\stackrel{\phi}{\sim}^{\mathrm{T}}(\mathbf{P}) & \underset{\sim}{0} \\
\underset{\sim}{\mathbf{0}} & \underset{\sim}{\phi^{\mathrm{T}}}(\mathbf{P})
\end{array}\right]
\end{aligned}
$$




$$
\underset{\sim}{\mathbf{X}^{N}}=\left\{\begin{array}{l}
\mathbf{X}_{1}^{1} \\
\mathbf{X}_{1}^{2} \\
\mathbf{X}_{2}^{1} \\
X_{2}^{2}
\end{array}\right\}
$$

$\mathrm{O}$ índice $\mathbf{N}$ é utilizado para indicar valores nodais. Assim, $\mathbf{x}_{\mathbf{i}}^{\mathbf{N}}$ representa a coordenada na direção $\mathbf{i}$ do ponto $\mathbf{N}$.

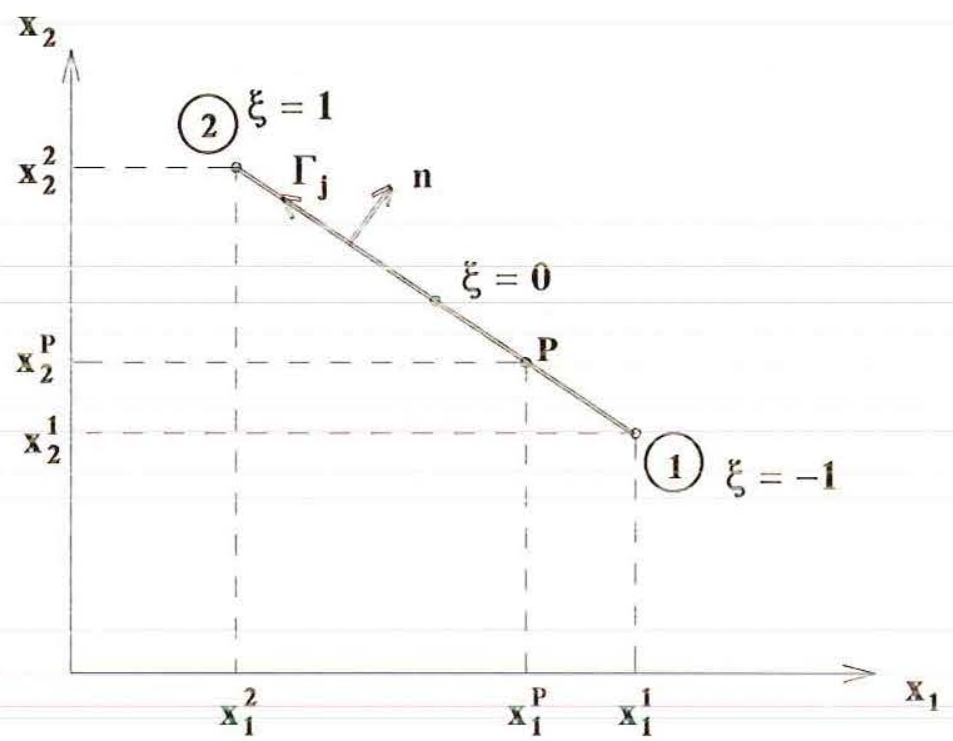

FIGURA 4.3 - Elemento de Contorno Linear.

O vetor das funções $\phi(\mathbb{P})$ é dado por:

$$
\underset{\sim}{\phi}(\mathbf{P})=\left\{\begin{array}{l}
\phi_{1}(\mathbb{P}) \\
\phi_{2}(\mathbf{P})
\end{array}\right\}
$$

onde $\phi_{\mathbf{i}}$ são funções da coordenada $\xi$, com origem no centro do elemento, expressas por:

$$
\phi_{1}(\mathrm{P})=\frac{1}{2} \cdot(1-\xi)
$$




$$
\phi_{2}(\mathbf{P})=\frac{1}{2} \cdot(1+\xi)
$$

Escrevendo-se explicitamente a eq.(4.1), resulta:

$$
\left\{\begin{array}{l}
\mathbf{x}_{1}(\mathbf{P}) \\
\mathbf{x}_{2}(\mathbf{P})
\end{array}\right\}=\left[\begin{array}{cccc}
\phi_{1}(\mathbf{P}) & \phi_{2}(\mathbf{P}) & 0 & 0 \\
0 & 0 & \phi_{1}(\mathbf{P}) & \phi_{2}(\mathbf{P})
\end{array}\right] \cdot\left\{\begin{array}{l}
\mathbf{X}_{1}^{1} \\
\mathbf{X}_{1}^{2} \\
\mathbf{X}_{2}^{1} \\
\mathbf{X}_{2}^{2}
\end{array}\right\}
$$

As funções $\phi_{\mathbf{i}}(\mathbf{i}=\mathbf{1 , 2})$ são conhecidas como funções de forma. Por definição, as funções de forma descrevem a variação da variável em questão através dos parâmetros nodais, isto é, elas indicam como as variáveis devem ser interpoladas entre seus parâmetros nodais.

\subsection{Aproximação das Variáveis}

Em cada elemento $\Gamma_{\mathbf{j}}$, os deslocamentos $-w$ (deslocamento vertical) e $\frac{\partial w}{\partial \mathbf{n}}$ (rotação) - e os esforços - $\mathbb{M}_{n}$ (momento de flexão) e $V_{n}$ (cortante equivalente) — são aproximados por funçôes, que estão associadas a um certo número de nós do elemento. Os valores das variáveis relativos aos nós, denominados "valores nodais", associam-se aos valores referentes aos elementos através das funções de forma.

Pode-se expressar os vetores de deslocamentos $\underset{\sim}{\mathbf{u}}$ e esforços $\mathbf{p}$ em um ponto $\mathbf{P}$, em termos de seus valores nodais $\underset{\sim}{\mathbf{U}^{N}}$ e $\mathbf{\sim}^{\mathrm{N}}$, através das relações:

$$
\begin{aligned}
& \underset{\sim}{\mathbf{u}}(\mathbf{P})=\left\{\begin{array}{c}
w \\
\frac{\partial w}{\partial \mathbf{n}}
\end{array}\right\}=\underset{\sim}{\underset{\Psi}{\Psi}}(\mathbf{P}) \cdot{\underset{\sim}{\mathbf{U}^{N}}}^{N}
\end{aligned}
$$

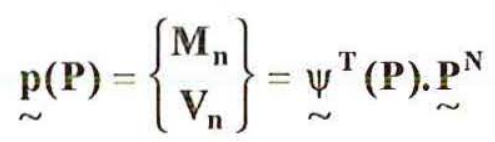


$\mathrm{O}$ número de valores nodais $\mathbf{N}$ e o tipo de função que compõe a matriz $\underset{\sim}{\underset{\sim}{T}}$ vão depender do nível de aproximação que se deseja.

As aproximações mais usuais, das variáveis nos elementos de contorno são:

- constantes;

- lineares $\left\{\begin{array}{l}\text { contínuas; } \\ \text { descontínuas; } \\ \text { mistas. }\end{array}\right.$

- quadráticas $\left\{\begin{array}{l}\text { contínuas; } \\ \text { descontínuas; } \\ \text { mistas. }\end{array}\right.$

\subsubsection{Aproximação Constante}

Para se ter a aproximação constante sobre um elemento a função aproximadora é definida apenas com um valor. O ponto correspondente é, geralmente, escolhido no meio do elemento, figura 4.4 . 


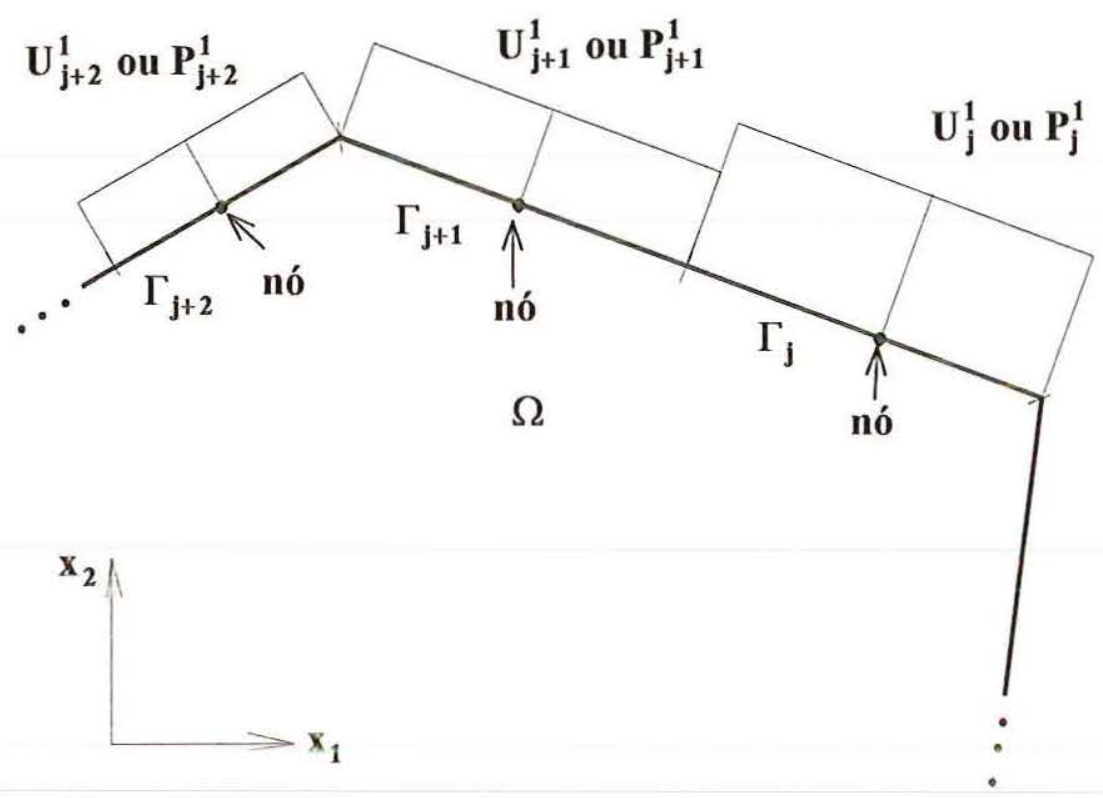

FIGURA 4.4 - Aproximação Constantes.

\subsubsection{Aproximação Linear Contínua}

A aproximação linear contínua é definida por função linear e não há descontinuidade entre elementos, figura 4.5.



FIGURA 4.5 - Aproximação Linear Contínua. 
Como os valores nodais de deslocamento $\left(\underset{\sim}{\mathbf{U}^{N}}\right)$ e de esforços $\left(\underset{\sim}{\mathbf{P}^{N}}\right)$ estão situados nos nós correspondentes às extremidades do elemento, figura 4.5 , as aproximações das variáveis podem ser feitas por funções $\underset{\sim}{\phi}$ idênticas à da geometria, isto é:

$$
\begin{aligned}
& \phi_{1}(P)=\frac{1}{2} \cdot(1-\xi) \\
& \phi_{2}(P)=\frac{1}{2} \cdot(1+\xi)
\end{aligned}
$$

com os valores nodais:

$$
\underset{\sim}{U^{N}}=\left\{\begin{array}{c}
w^{1} \\
\left(\frac{\partial w}{\partial \mathrm{n}}\right)^{1} \\
w^{2} \\
\left(\frac{\partial w}{\partial \mathrm{n}}\right)^{2}
\end{array}\right\}
$$

$$
\underset{\sim}{\mathbf{P}^{N}}=\left\{\begin{array}{c}
\mathbf{M}_{n}^{1} \\
\mathbf{V}_{n}^{1} \\
\mathbf{M}_{n}^{2} \\
\mathbf{V}_{n}^{2}
\end{array}\right\}
$$

Com isso, as equações (4.8.a) e (4.8.b), que expressam os deslocamentos u e esforços $\mathbf{p}$ em um ponto $\mathbf{P}$, em função de seus valores nodais $\underset{\sim}{\mathbf{U}^{N}}$ e ${\underset{\sim}{\mathbb{P}}}^{N}$, podem ser escritas explicitamente como:

$$
\underset{\sim}{\mathbf{u}}(\mathbf{P})=\left\{\begin{array}{c}
w(\mathbf{P}) \\
\frac{\partial w}{\partial \mathbf{n}}(\mathbf{P})
\end{array}\right\}=\left[\begin{array}{cccc}
\phi_{1}(\mathbf{P}) & 0 & \phi_{2}(\mathbf{P}) & 0 \\
0 & \phi_{1}(\mathbf{P}) & 0 & \phi_{2}(\mathbf{P})
\end{array}\right] \cdot\left\{\begin{array}{c}
w^{1} \\
\left(\frac{\partial w}{\partial \mathbf{n}}\right)^{1} \\
w^{2} \\
\left(\frac{\partial w}{\partial \mathbf{n}}\right)^{2}
\end{array}\right\}
$$




$$
\underset{\sim}{\mathbf{p}(\mathbf{P})}=\left\{\begin{array}{c}
\mathbf{M}_{\mathbf{n}}(\mathbf{P}) \\
\mathbf{V}_{\mathrm{n}}(\mathbf{P})
\end{array}\right\}=\left[\begin{array}{cccc}
\phi_{1}(\mathbf{P}) & \mathbf{0} & \phi_{2}(\mathbf{P}) & \mathbf{0} \\
0 & \phi_{1}(\mathbf{P}) & 0 & \phi_{2}(\mathbf{P})
\end{array}\right] \cdot\left\{\begin{array}{c}
\mathbf{M}_{n}^{1} \\
\mathbf{V}_{n}^{1} \\
\mathbf{M}_{n}^{2} \\
\mathbf{V}_{n}^{2}
\end{array}\right\}
$$


FIGURA 4.6 - Funções de Forma para Aproximação Linear Contínua das Variáveis.

A presença de descontinuidade das funções aproximadoras devido a angulosidade ou vinculações diferentes no contorno de uma placa, figura 4.7, torna necessária a descontinuidade das variáveis nos casos práticos, podendo-se assim, definir outros tipos de elementos com diferentes aproximações das variáveis, de modo a expressar tal descontinuidade. 


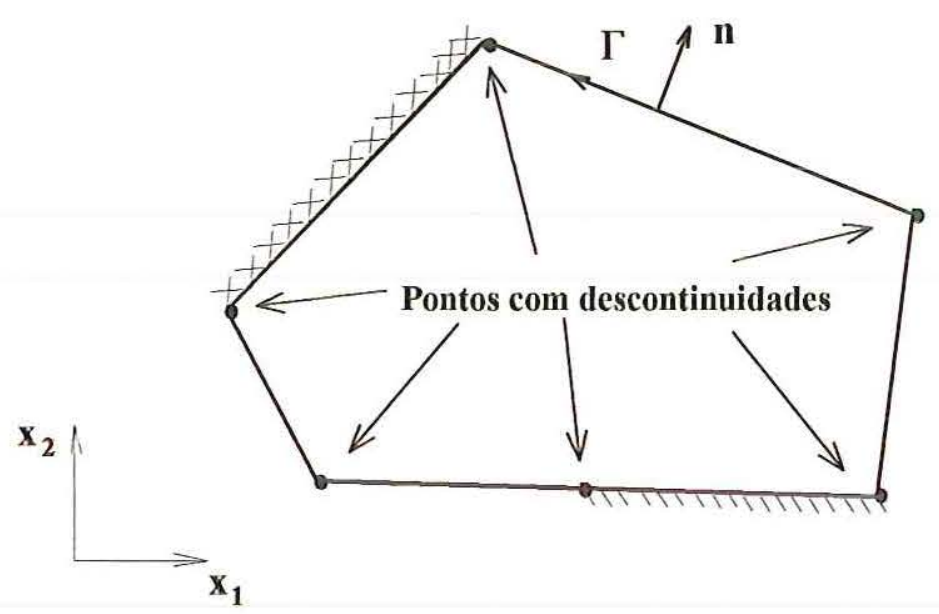

FIGURA 4.7 - Descontinuidade do Contorno de uma Placa.

\subsubsection{Aproximação Linear Descontínua}

Os elementos lineares com aproximações descontínuas das variáveis se caracterizam por expressar uma aproximação linear entre dois nós e permitir que haja descontinuidade das variáveis (deslocamentos e esforços) entre dois elementos. Uma das maneiras de se obter essa representação descontínua é através da definição de nós no interior do elemento. Neste caso, as variáveis $\underset{\sim}{u}(\mathbf{P})$ e $\underset{\sim}{p(P)}$ serão aproximadas

pelas eq.(4.12) e (4.13), respectivamente, sendo as funções $\phi$ agora dadas por:

$$
\begin{gathered}
\phi_{1}(\mathbf{P})=\frac{1}{\xi_{2}-\xi_{1}} \cdot\left(\xi_{2}-\xi\right) \\
\phi_{2}(\mathbf{P})=\frac{1}{\xi_{1}-\xi_{2}} \cdot\left(\xi_{1}-\xi\right)
\end{gathered}
$$

onde, conforme a figura $4.8, \xi_{1}$ e $\xi_{2}$ são as posições dos nós com relação ao centro do elemento, em coordenadas homogêneas; $\xi$ é a coordenada homogênea do ponto considerado e; $\phi_{1}$ e $\phi_{2}$ são funções de forma. 


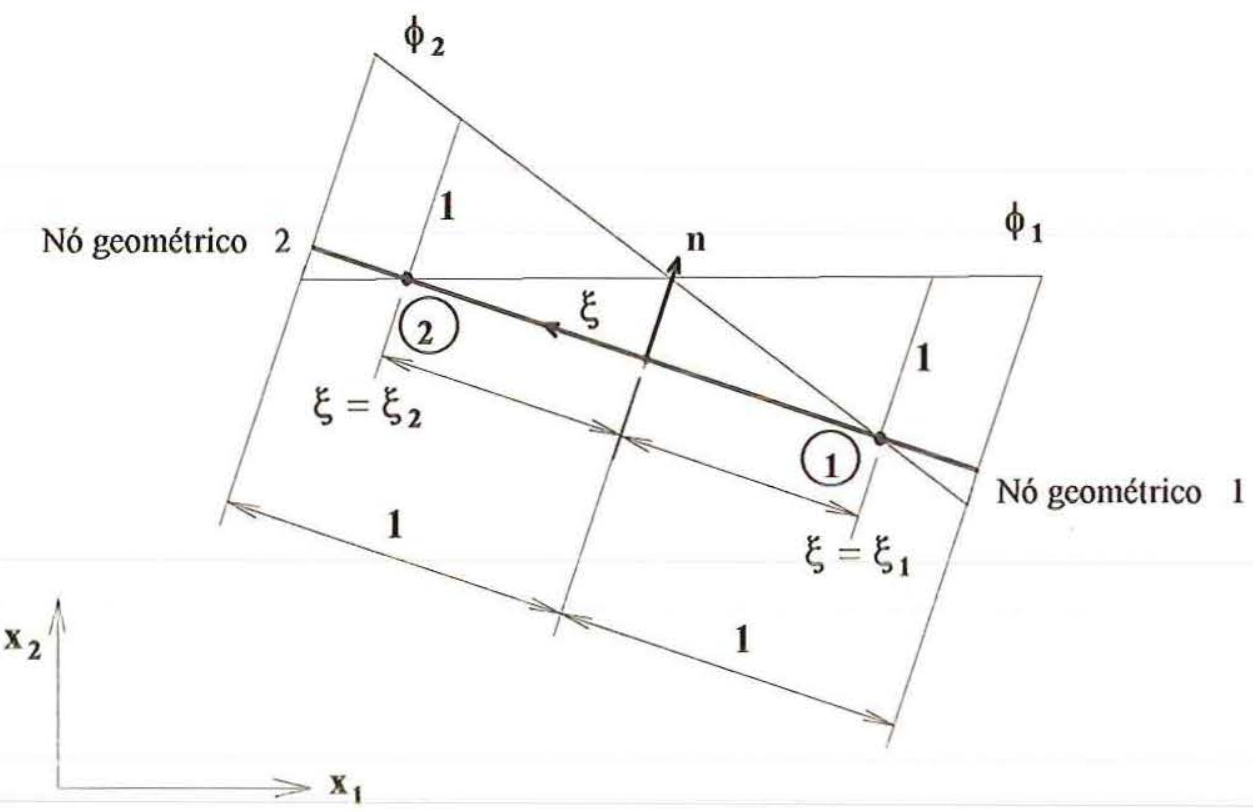

FIGURA 4.8 - Função de Forma Linear Descontínua para as Variáveis.

As funções aproximadoras (4.14) e (4.15) estão relacionadas aos nós (1) e (2) definidos em pontos internos do elemento, garantindo-se assim, a descontinuidade das variáveis nas extremidades.

Para o caso particular em que os pontos nodais (1) e (2) coincidem, respectivamente, com os extremos geométricos 1 e 2 do elemento (figura 4.8), isto é , $\xi_{1}=-1$ e $\xi_{2}=1$, as expressões (4.14) e (4.15) ficam iguais às obtidas considerando aproximação linear contínua, equações (4.9.a) e (4.9.b).

\subsubsection{Aproximação Linear Mista}

As aproximações mistas consistem de combinações entre aproximações contínuas e descontínuas e são utilizadas quando se pretende expressar descontinuidade em uma das extremidades do elemento, figura 4.9.

A utilização de apenas elementos descontínuos, na discretização do contorno de uma placa, aumenta significativamente o número de nós e por conseqüência o número de equações do sistema. Quando utilizado apenas para modular descontinuidade em vértices ou em pontos onde existe mudança das condições de 
contorno, torna-se vantajosa a sua combinação com os elementos com aproximações contínuas e mistas das variáveis, GIL RODRIGUES $(1985,1986)$.
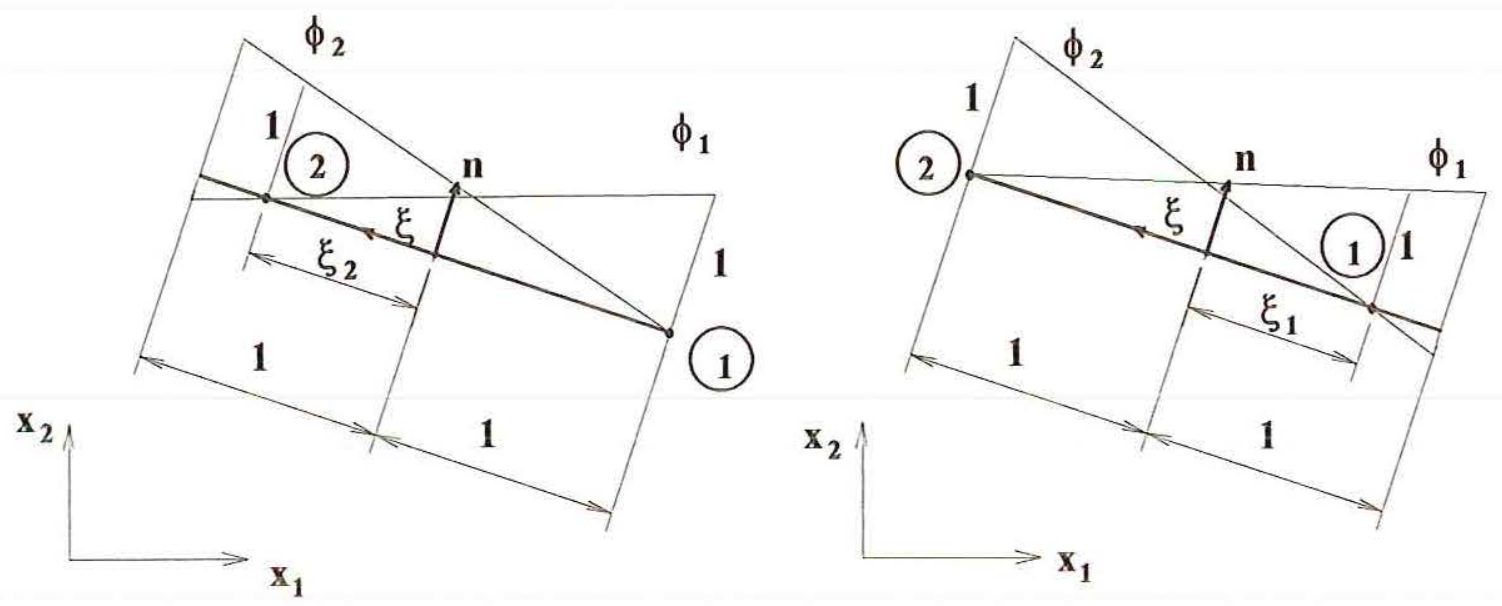

FIGURA 4.9 - Função de Forma Linear Mista para as Variáveis.

\subsubsection{Aproximação Quadrática Contínua}

Esta aproximação requer três pontos nodais por elemento, figura 4.10. São usadas funções polinomiais quadráticas para a aproximação das variáveis em cada elemento. Não deve haver descontinuidade das variáveis envolvidas em um nó associado a dois elementos adjacentes. 


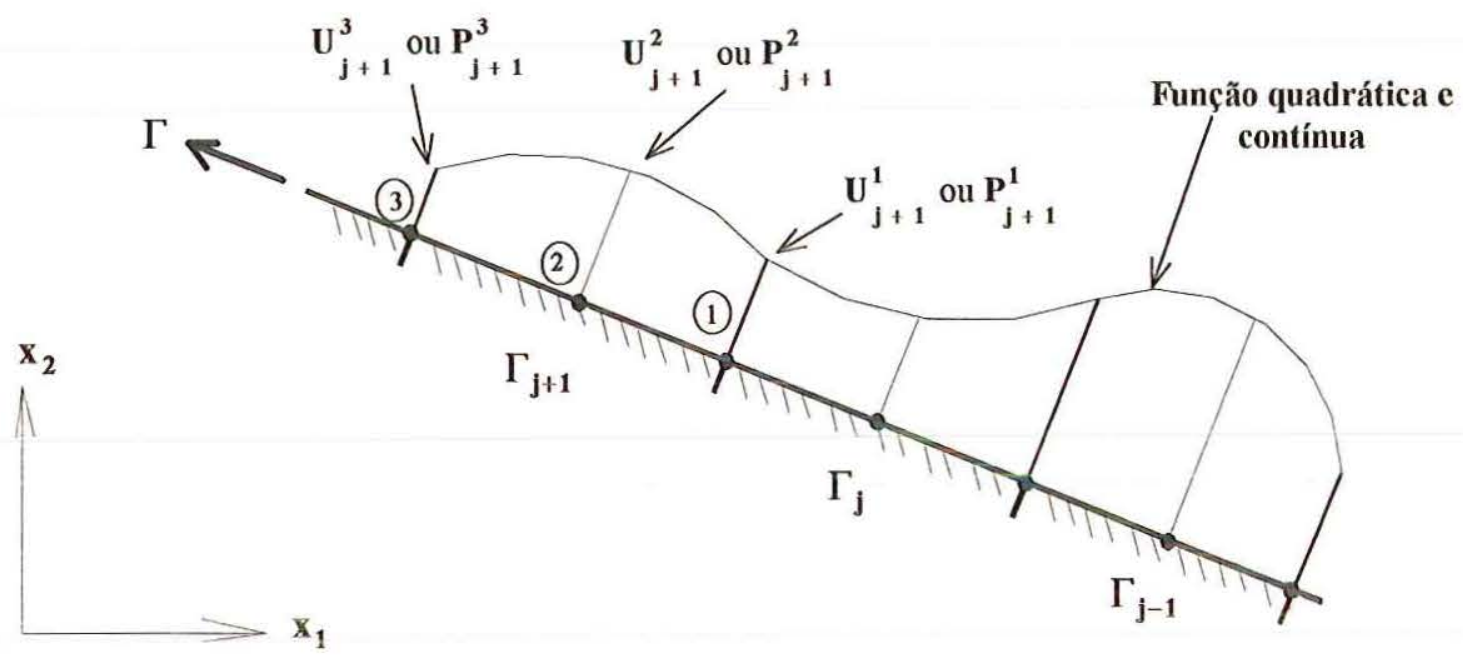

FIGURA 4.10 - Aproximação Quadrática Contínua das Variáveis no Contorno.

Assim, pode-se expressar as variáveis $\underset{\sim}{\mathbf{u}}$ e p (deslocamentos e esforços) sobre cada elemento, através de funções aproximadoras do segundo grau. É necessário relacionar as variáveis de um ponto qualquer $\mathbf{P}$ do elemento com os valores nodais destas variáveis $\underset{\sim}{\mathbf{U}^{N}}$ e ${\underset{\sim}{\mathbf{P}}}^{N}$. Para isto, usam-se as relações (4.8.a) e (4.8.b), sendo as funções de forma $\phi_{\mathbf{i}}(\mathrm{i}=1,2,3)$ dadas por:

$$
\begin{gathered}
\phi_{1}=-\frac{1}{2} \cdot(1-\xi) \cdot \xi \\
\phi_{2}=1-\xi^{2} \\
\phi_{3}=\frac{1}{2} \cdot(1+\xi) \cdot \xi
\end{gathered}
$$


com os valores nodais:

$$
\begin{aligned}
\underset{\sim}{\mathbf{U}^{N}} & =\left\{\begin{array}{c}
w^{1} \\
\left(\frac{\partial w}{\partial n}\right)^{1} \\
w^{2} \\
\left(\frac{\partial w}{\partial n}\right)^{2} \\
w^{3} \\
\left(\frac{\partial w}{\partial n}\right)^{3}
\end{array}\right\} \\
\underset{\sim}{\mathbf{P}^{N}} & =\left\{\begin{array}{c}
\mathbf{V}_{n}^{1} \\
\mathbf{M}_{n}^{1} \\
\mathbf{V}_{n}^{2} \\
\mathbf{M}_{n}^{2} \\
\mathbf{V}_{n}^{3} \\
\mathbf{M}_{n}^{3}
\end{array}\right\}
\end{aligned}
$$

Com isso, as equações (4.8.a) e (4.8.b), que expressam os deslocamentos $\underset{\sim}{\text { u }} \mathrm{e}$ esforços $\mathbf{p}$ em um ponto $\mathbf{P}$, em função de seus valores nodais $\underset{\sim}{\mathbf{U}^{N}}$ e $\mathbf{P}^{\mathbf{N}}$, podem ser escritos explicitamente como:

$$
\underset{\sim}{u}(\mathbf{P})=\left\{\begin{array}{c}
w(\mathbf{P}) \\
\frac{\partial w}{\partial \mathbf{n}}(\mathbf{P})
\end{array}\right\}=\left[\begin{array}{cccccc}
\phi_{1}(\mathbf{P}) & 0 & \phi_{2}(\mathbf{P}) & 0 & \phi_{3}(\mathbf{P}) & 0 \\
0 & \phi_{1}(\mathbf{P}) & 0 & \phi_{2}(\mathbf{P}) & 0 & \phi_{3}(\mathbf{P})
\end{array}\right] \cdot\left\{\begin{array}{c}
w^{2} \\
\left(\frac{\partial w}{\partial \mathbf{n}}\right)^{2} \\
w^{3} \\
\left(\frac{\partial w}{\partial \mathrm{n}}\right)^{3}
\end{array}\right\}
$$




$$
\underset{\sim}{\mathbf{p}(\mathbf{P})}=\left\{\begin{array}{l}
\mathbf{M}_{\mathbf{n}}(\mathbf{P}) \\
\mathbf{V}_{\mathbf{n}}(\mathbf{P})
\end{array}\right\}=\left[\begin{array}{cccccc}
\phi_{1}(\mathbf{P}) & \mathbf{0} & \phi_{2}(\mathbf{P}) & \mathbf{0} & \phi_{3}(\mathbf{P}) & \mathbf{0} \\
0 & \phi_{1}(\mathbf{P}) & 0 & \phi_{2}(\mathbf{P}) & 0 & \phi_{3}(\mathbf{P})
\end{array}\right] \cdot\left\{\begin{array}{l}
\mathbf{M}_{n}^{1} \\
\mathbf{V}_{n}^{1} \\
\mathbf{M}_{n}^{2} \\
\mathbf{V}_{n}^{2} \\
\mathbf{M}_{n}^{3} \\
\mathbf{V}_{n}^{3}
\end{array}\right\}
$$
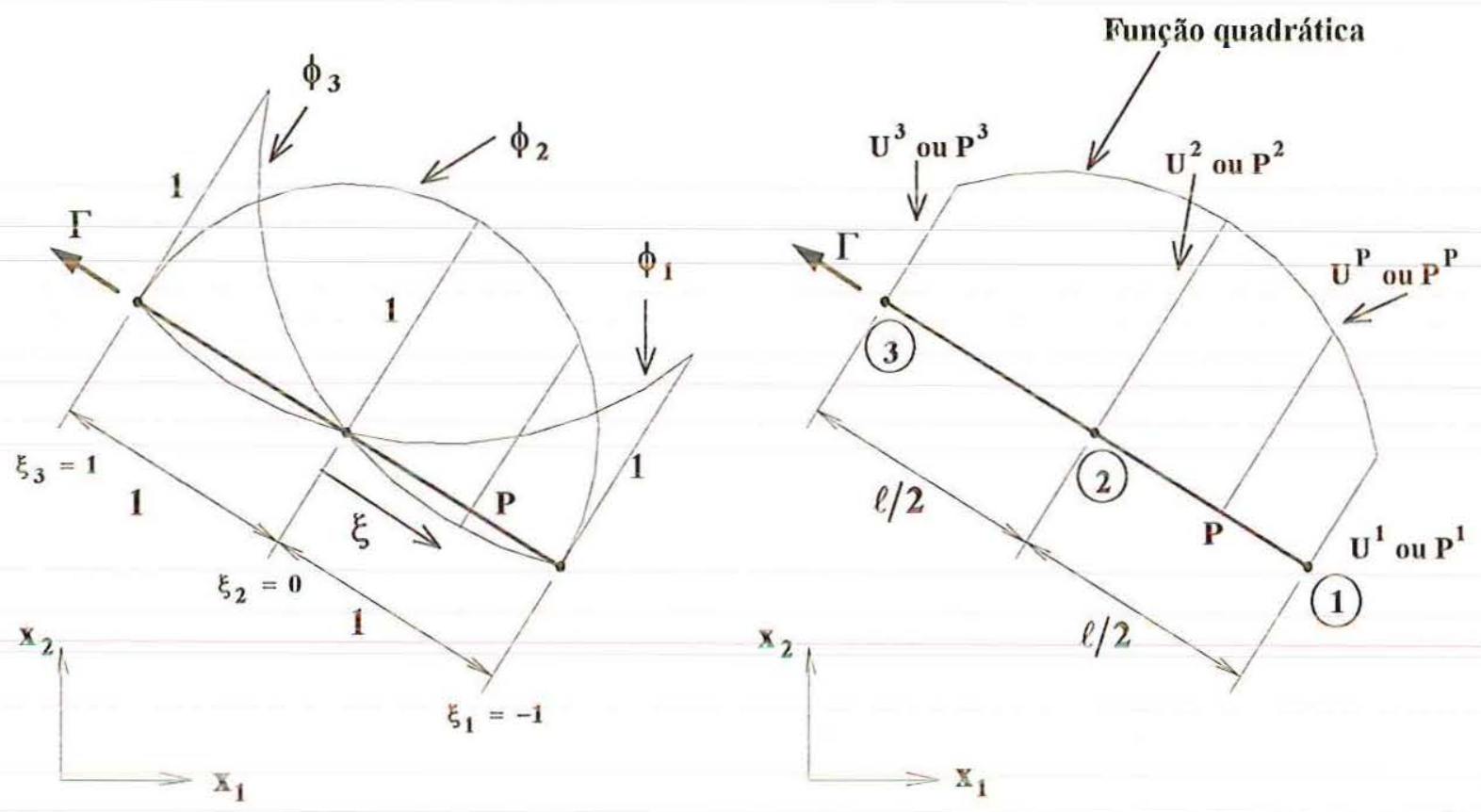

FIGURA 4.11 - Funções de Forma para Aproximação Quadrática Continua das Variáveis.

\subsubsection{Aproximação Quadrática Descontínua}

Esta aproximação permite que haja descontinuidade das variáveis envolvidas entre dois elementos adjacentes. Estes pontos nodais podem estar em qualquer posição ao longo do elemento, permitindo que os nós(1) (3) da figura 4.11 passem a ter coordenadas locais $\xi_{1}$ e $\xi_{3}$, respectivamente, e o nó(2) localizado no centro do elemento ainda permance com $\xi_{2}=\mathbf{0}$, conforme indicado na figura 4.12. 
Para esta aproximação as três funções interpoladoras $\phi_{1}, \phi_{2}$ e $\phi_{3}$, são dadas por:

$$
\begin{gathered}
\phi_{1}(\mathbf{P})=\frac{1}{\xi_{1}\left(\xi_{3}-\xi_{1}\right)} \cdot\left(\xi_{3}-\xi\right) \cdot \xi \\
\phi_{2}(\mathbf{P})=1-\frac{\xi_{3}+\xi_{1}}{\xi_{3} \cdot \xi_{1}} \cdot \xi+\left(\xi_{3}-\xi\right) \cdot \frac{1}{\xi_{1} \cdot \xi_{3}} \cdot \xi^{2} \\
\phi_{3}(\mathbf{P})=\frac{1}{\xi_{3}\left(\xi_{1}-\xi_{3}\right)} \cdot\left(\xi_{1}-\xi\right) \cdot \xi
\end{gathered}
$$
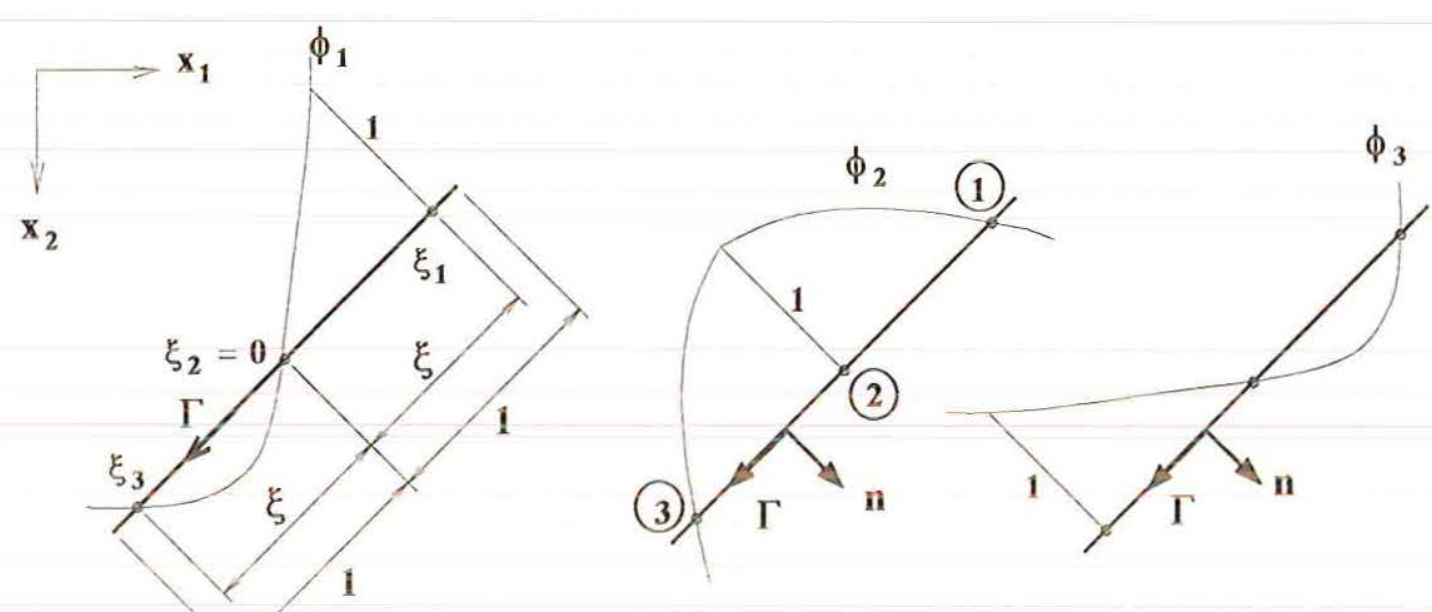

FIGURA 4.12 - Funções Interpoladoras Quadráticas Descontínuas.

\subsubsection{Aproximação Quadrática Mista}

Como já visto no item 4.3.4, a aproximação mista é usada quando se necessita da combinação de aproximações contínuas e descontínuas, bastando, para isso, adotar valores de $\xi_{1}=-1$ ou $\xi_{3}=1$ nas funções interpoladoras (4.21). 


\subsubsection{Aproximação Adotada}

Neste trabalho será adotado o elemento de contorno com geometria linear com aproximação quadrática contínua para as variáveis. Para isso, é necessário que o elemento linear tenha três pontos nodais, como definido no item 4.3.5.

Nos pontos onde existirem descontinuidades, das variáveis, das vinculações ou descontinuidade da geometria como o canto, será utilizado o artifício do nó duplo para simular esta descontinuidade, figura 4.13. Os nós duplos caracterizam-se por apresentarem mesma coordenadas, porém permitindo que haja descontinuidade.

Como já foi mencionado, cada ponto de colocação está associado a uma equação integral de deslocamento, que será transformada em equação algébrica após a discretização do contorno, dando origem a uma linha do sistema de equações. Com a existência de nó duplo, pelo fato de terem mesmas coordenadas, serão geradas duas equações algébricas que poderão, em função das condições de contorno, ser linearmente dependentes e, com isso, o sistema de equações será singular.

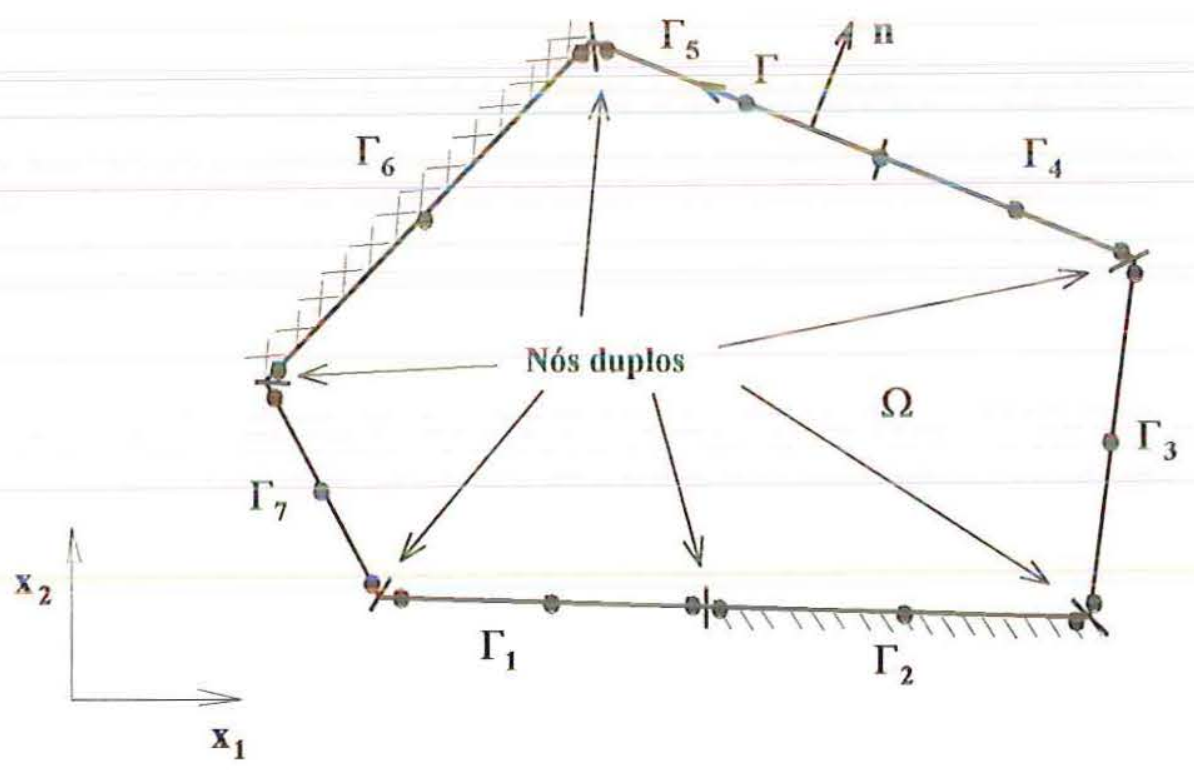

FIGURA 4.13 - Nós Duplos. 
Para evitar a singularidade provocada pelo nó duplo, cria-se um ponto de colocação, no interior do elemento, figura 4.14. Com o ponto de colocação deslocado monta-se a equação algébrica correspondente. Como as incógnitas estão expressas nas extremidades e no meio do elemento, tem-se a necessidade de representar o deslocamento do ponto de colocação no interior do elemento, em termos dos valores nodais. Para isso, utilizam-se as funções de forma já definidas anteriormente, resultando então:

$$
w(\bar{Q})=\left\{\begin{array}{lll}
\phi_{1} & \phi_{2} & \phi_{3}
\end{array}\right\} \cdot\left\{\begin{array}{l}
w^{1} \\
w^{2} \\
w^{3}
\end{array}\right\}
$$

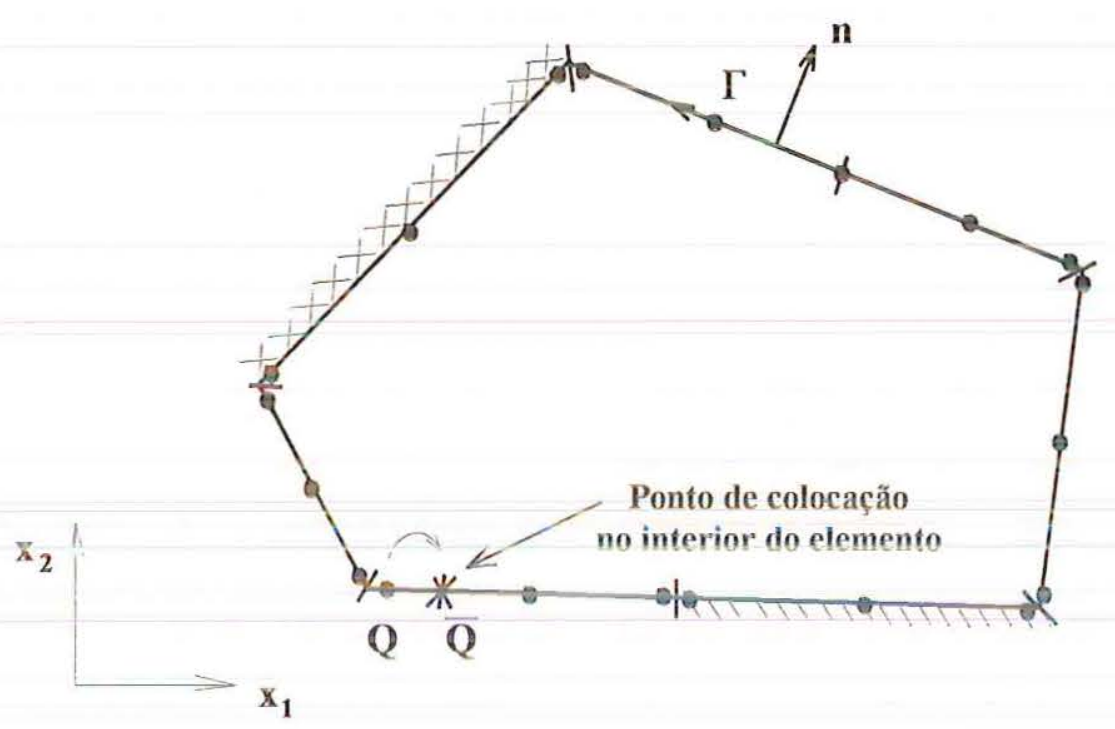

FIGURA 4.14 - Ponto de Colocação.

\subsection{Integrais de Domínio}

Na equação (3.55), que representa a equação integral do deslocamento para placa com espessura constante, aparece uma integral de domínio, sobre a região do carregamento $\Omega_{\mathrm{g}}$, dada por: 


$$
\int_{\Omega_{g}} g(p) \cdot w^{*}(Q, p) \cdot d \Omega_{g}(p)
$$

A integral (4.22) tem valor numérico conhecido, pois não envolve nenhuma incógnita. $\mathbf{g ( p )}$ corresponde ao carregamento distribuído, que, neste trabalho, será assumido linear sobre a região $\Omega_{\mathrm{g}}$, figura 4.15. $w^{\text {* }}$ representa a solução fundamental de deslocamento, fornecida pela eq.(2.80).

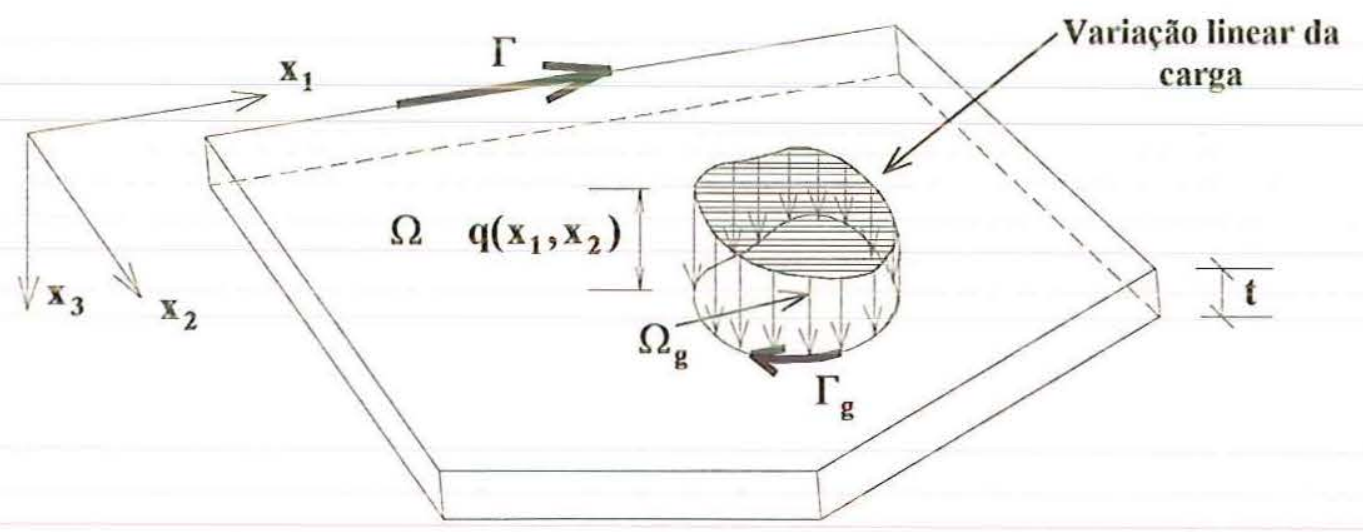

FIGURA 4.15 - Placa com Carregamento Distribuído.

Existem vários métodos numéricos para o cálculo da integral (4.22). Dentre eles, pode-se citar o Método de Monte Carlo que utiliza números randômicos (aleatórios). Pode-se usar também uma extensão da quadratura de Gauss para obter integrais múltiplas. Como terceira alternativa, a qual foi adotada neste trabalho, podese transformar a integral de domínio em uma integral sobre o contorno do carregamento (integral de linha).

Já na equação integral do deslocamento, para o caso de placa com variação de espessura, eq.(3.58), além da integral de domínio (4.22), surge outra integral de domínio dada por: 


$$
\int_{\Omega} \frac{1}{D_{0}} \cdot\left[2 \cdot \frac{\partial D(s)}{\partial x_{i}} \cdot q_{i}^{*}+\frac{\partial^{2} D(s)}{\partial x_{i} \cdot \partial x_{j}} \cdot m_{i j}^{*}(Q, p)\right] \cdot w(p) \cdot d \Omega
$$

Esta integral contém uma incógnita do problema, o deslocamento $w(\mathbf{p})$. Para resolver esta integral será necessário dividir o domínio em células onde a função deslocamento será aproximada por uma função polinomial. Neste trabalho, admitiramse células com geometria triangular e função interpoladora linear do deslocamento no domínio, figura 4.16 .

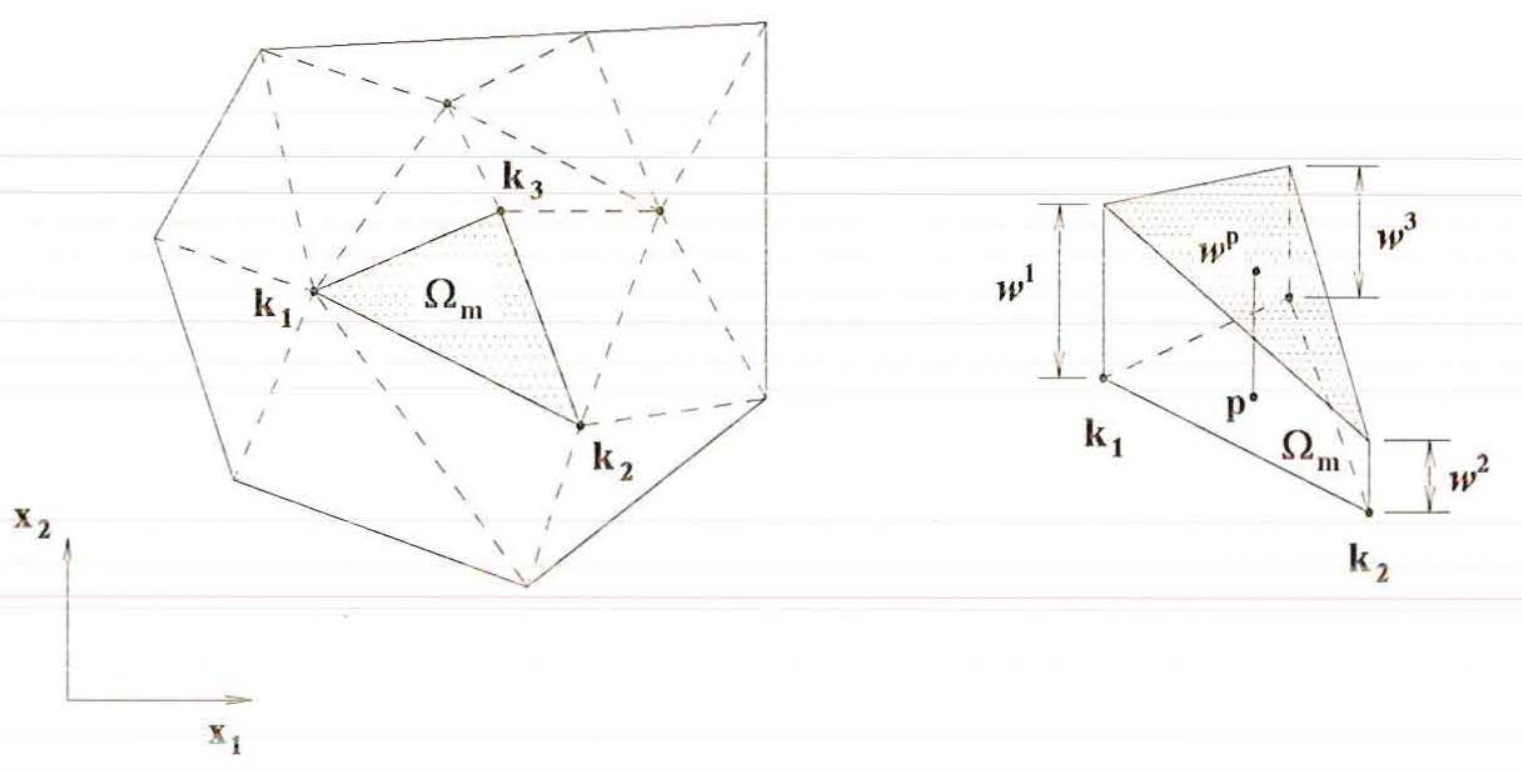

FIGURA 4.16 - Divisão do Domínio em Células Triangulares.

\subsubsection{Integrais de Domínio para o Carregamento}

Para transformar a integral de domínio (4.22) em uma integral de contorno, considere-se a placa da figura 4.17 , submetida a um carregamento " $\mathrm{g}$ " distribuído numa região $\Omega_{\mathrm{g}}$ de contorno $\Gamma_{\mathrm{g}}$ e o ponto de carregamento " $q$ ". A partir desta figura, obtêm-se as seguintes relações: 


$$
\begin{aligned}
& d \Omega_{g}=r \cdot d r . d \theta \\
& d \theta=\frac{r, n_{i} \cdot n_{i}}{R} \cdot d \Gamma_{g}
\end{aligned}
$$

Com isso, pode-se obter:

$$
d \Omega_{g}=r \cdot d r \cdot \frac{r_{i} \cdot n_{i}}{R} \cdot d \Gamma_{g}
$$

onde $\mathbf{r}_{\mathrm{i}}$ e $\mathbf{n}_{\mathbf{i}}$ representam os co-senos diretores do vetor $\mathbf{r}$ e da normal $\mathbf{n}$, ao contorno da região do carregamento.

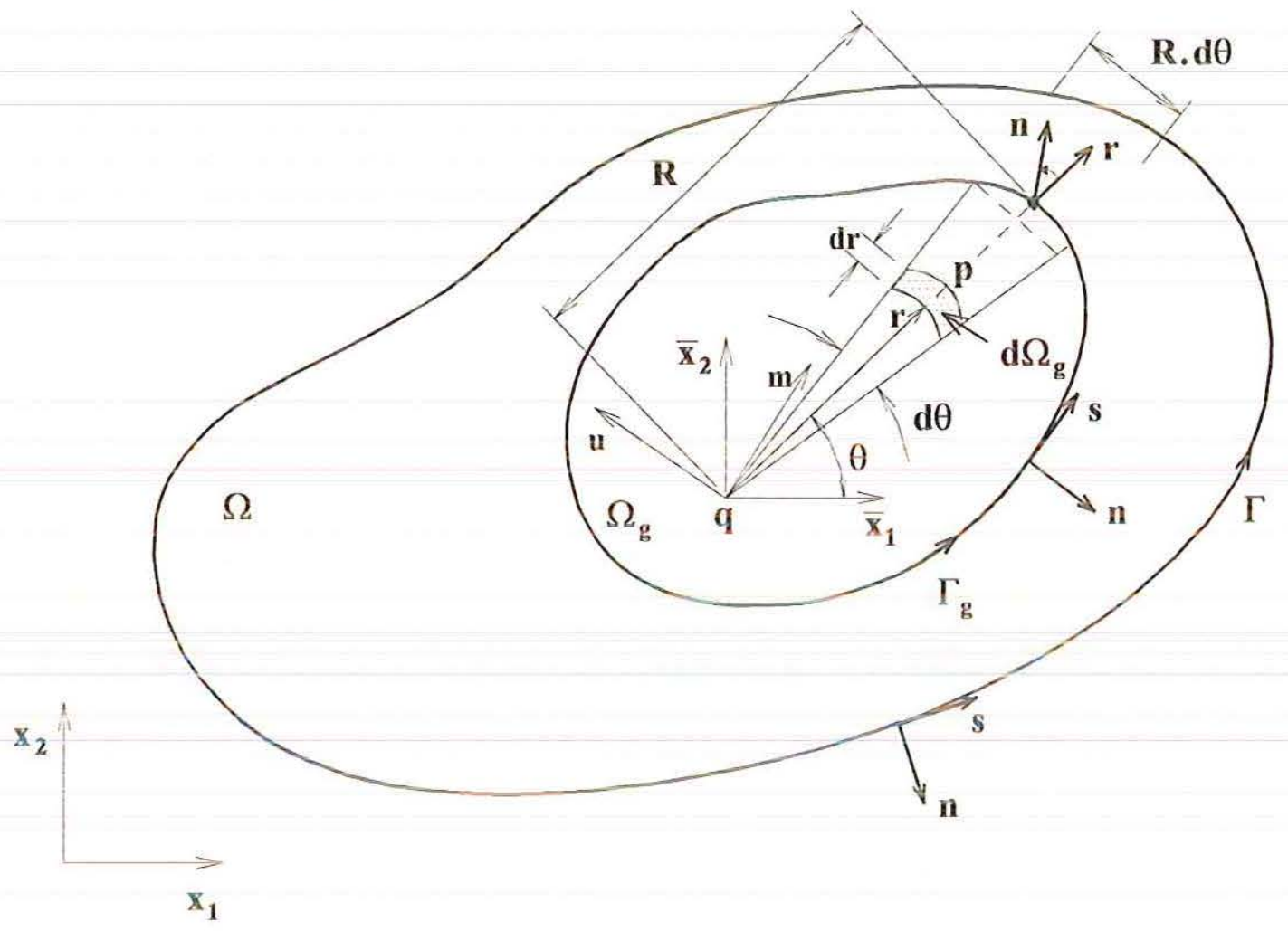

FIGURA 4.17 - Região Carregada $\Omega_{\mathrm{g}}$.

Em conseqüência da mudança de variável indicada em (4.26), a integral de domínio de (4.22) é expressa por: 


$$
\int_{\Gamma_{\mathrm{g}}}^{\mathrm{R}} \int_{0}^{\mathrm{g}} \mathrm{g}(\mathrm{p}) \cdot w^{*}(\mathrm{q}, \mathrm{p}) \cdot \frac{\mathrm{r},{ }_{\mathrm{i}} \cdot \mathrm{n}_{\mathrm{i}}}{\mathrm{R}} \cdot \mathrm{r} \cdot \mathrm{dr} \cdot \mathrm{d} \Gamma_{\mathrm{g}}
$$

onde $\mathbf{R}$ é o valor de $\mathbf{r}$ para um ponto qualquer do contorno $\Gamma_{\mathrm{g}}$, figura 4.17.

Tendo-se admitido que a carga $\mathrm{g}$ varia linearmente na região $\Omega_{\mathrm{g}}$, a expressão de $\mathbf{g}(\mathbf{p})$ em relação ao sistema de coordenadas cartesianas $\left(\mathbf{x}_{1}, \mathbf{x}_{2}\right)$ é dada por:

$$
g(p)=A \cdot x_{1}(p)+B \cdot x_{2}(p)+C
$$

Em relação ao sistema $(\bar{x}, \bar{y})$, de origem em " $q$ ", figura 4.17 , tem-se:

$$
\left\{\begin{array}{l}
x_{1}(p)=x_{1}(q)+\bar{x}_{1}(p) \\
x_{2}(p)=x_{2}(q)+\bar{x}_{2}(p)
\end{array}\right.
$$

Substituindo-se (4.29) em (4.28), resulta:

$$
g(p)=A \cdot x_{1}(q)+B \cdot x_{2}(q)+C+A \cdot \bar{x}_{1}(p)+B \cdot \bar{x}_{2}(p)=g(q)+A \cdot \bar{x}_{1}(p)+B \cdot \bar{x}_{2}(p)
$$

onde $g(q)$ é o valor da intensidade da carga no ponto " $q$ ".

Substituindo-se em (4.27) a eq.(4.30) em coordenadas polares e o valor de $w^{*}(\mathfrak{q}, \mathbf{p})$ dado pela relação $(2.39)$, obtém-se:

$$
\int_{\Gamma_{g}}\left[\int_{0}^{R}(g(q)+A \cdot r \cdot \cos (\theta)+B \cdot r \cdot \operatorname{sen}(\theta)) \cdot \frac{r^{2}}{8 \cdot \pi \cdot D_{0}} \cdot\left(\ln (r)-\frac{1}{2}\right) \cdot r \cdot d r\right] \cdot \frac{r_{1} \cdot n_{i}}{R} \cdot d \Gamma_{g}
$$


Resolvendo-se a integral interna, em r, de (4.31), resulta:

$$
\begin{aligned}
& \int_{\Omega_{g}} g(p) \cdot w^{*}(q, p) \cdot d \Omega_{g}(p)=\frac{g(q)}{32 \cdot \pi \cdot D_{0}} \cdot \int_{\Gamma_{g}} R^{3} \cdot\left(\ln (R)-\frac{3}{4}\right) \cdot r_{i_{i}} \cdot n_{i} \cdot d \Gamma_{g} \\
& \quad+\frac{1}{40 . \pi \cdot D_{0}} \cdot \int_{\Gamma_{g}} R^{4} \cdot\left(\ln (R)-\frac{7}{10}\right) \cdot(A \cdot \cos (\theta)+B \cdot \operatorname{sen}(\theta)) \cdot r,_{i} \cdot n_{i} \cdot d \Gamma_{g}
\end{aligned}
$$

Uma outra alternativa para a transformação das integrais de domínio em integrais no contomo é a utilização do Método da Reciprocidade Múltipla, sugerido por NOWAK \& BREBBIA (1989).

Para os casos de uma carga $\mathbf{g ( p )}$ qualquer, a integral (4.32) pode ser resolvida dividindo-se a região $\Omega_{\mathrm{g}}$ em subregiões menores, ou células, e fazendo-se o somatório das integrais sobre todas as células do domínio SILVA (1988).

Analogamente à discretização do contorno da placa, como no item 4.2 podese discretizar também o contorno do carregamento em elementos de contorno linear, figura 4.18, sendo válidas as relações (4.1) a (4.7). Devido a esta discretização, podese afirmar que:

$$
\int_{\Gamma_{g}} F\left(\Gamma_{g}\right) \cdot d \Gamma_{g}=\sum_{i=1}^{N e l c c} \int_{\Gamma_{g i}} F\left(\Gamma_{g i}\right) \cdot d \Gamma_{g i}
$$




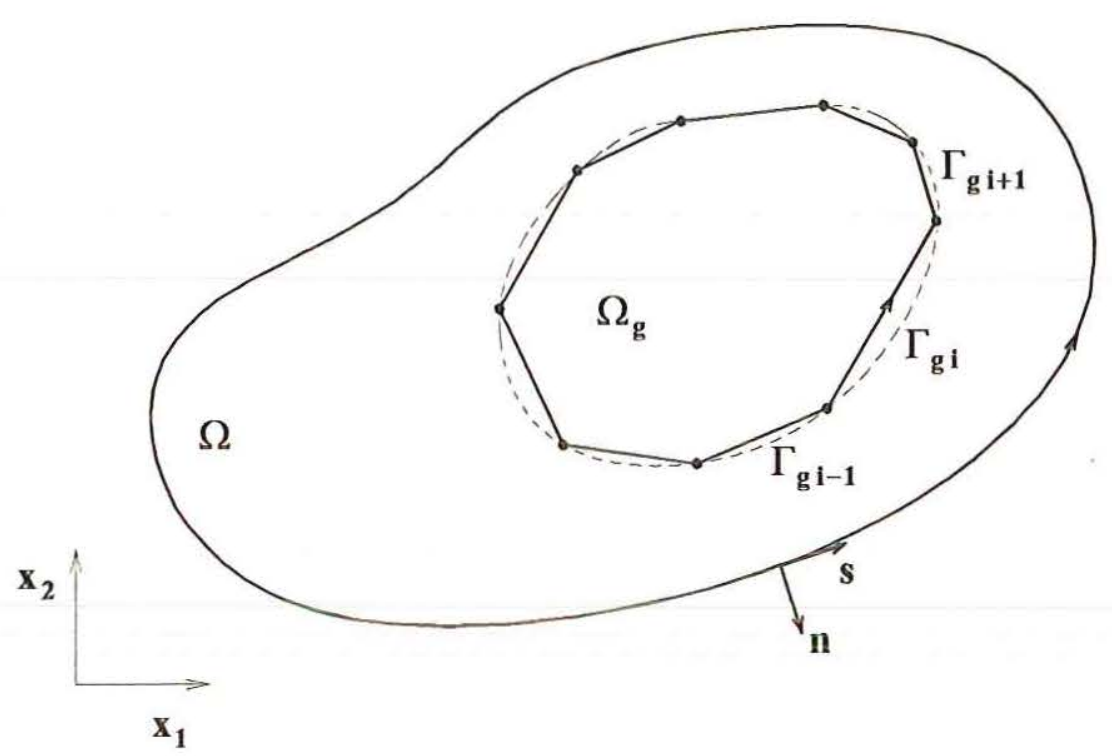

FIGURA 4.18 - Discretização do Contorno do Carregamento.

\subsection{2 integrais de Domínio sobre as Células}

Discretizando-se o domínio da placa em células internas, figura 4.19a, os deslocamentos poderão ser aproximados, transformando-se a integral (4.23) em somatório de integrais sobre estas células, obtendo-se a seguinte expressão:

$$
\sum_{i=1}^{N c d} \int_{\Omega_{\mathrm{gsi}}} \bar{K}^{*}(\mathrm{q}, \mathrm{p}), w(\mathrm{p}) \cdot \mathrm{d} \Omega_{\mathrm{gsi}}(\mathrm{p})
$$

sendo o valor de $\overline{\mathbf{K}}^{*}(\mathbf{q}, \mathbf{p})$ dado por:

$$
\bar{K}^{*}(q, p)=\frac{1}{D_{0}} \cdot\left[2 \cdot \frac{\partial D(s)}{\partial x_{i}} \cdot q_{i}^{*}(q, p)+2 \cdot \frac{\partial^{2} D(s)}{\partial x_{i} \cdot \partial x_{j}} \cdot m_{i j}^{*}(q, p)\right]
$$




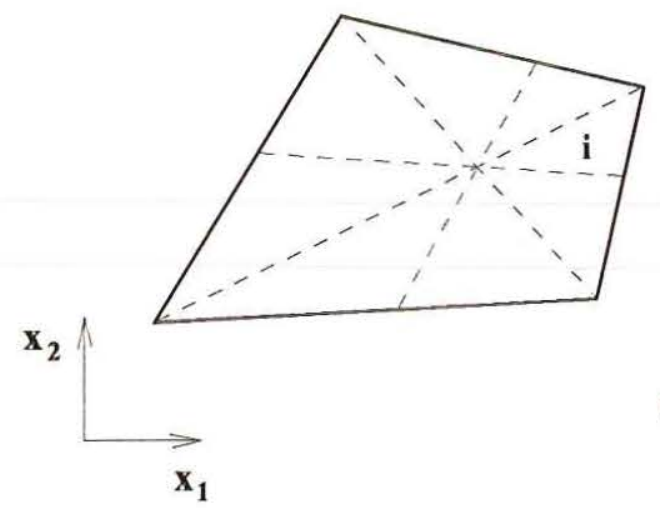

(a)

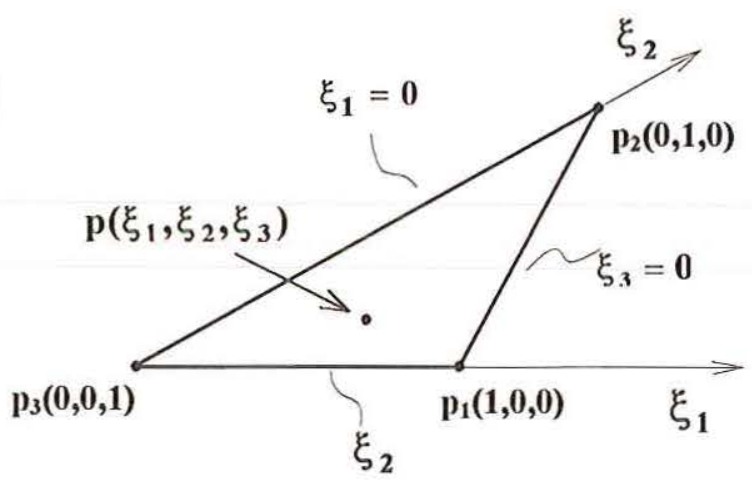

(b)

FIGURA 4.19 - Definição do Sistema de Coordenadas de uma Células Triangular " $\mathrm{".}$

Um ponto " $\mathbf{p}$ " de coordenadas $\left(\mathbf{x}_{\mathbf{1}}^{\mathbf{p}}, \mathbf{x}_{2}^{\mathbf{p}}\right)$ pode ser definido em função das coordenadas dos vértices $p_{1}, p_{2}$ e $p_{3}$ de uma célula triangular "i" , figura $4.19 \mathrm{~b}$, da seguinte forma:

$$
{\underset{\sim}{p}}^{\mathrm{p}}=\underset{\sim}{\Psi^{\mathrm{T}}} \cdot \underset{\sim}{\mathrm{X}^{\mathrm{N}}}
$$

onde:

$$
\begin{gathered}
\underset{\sim}{\mathrm{x}^{\mathrm{p}}}=\left\{\begin{array}{c}
\mathrm{x}_{1}^{\mathrm{p}} \\
\mathrm{x}_{2}^{\mathrm{p}}
\end{array}\right\} \\
\underset{\sim}{\Psi^{\mathrm{T}}}=\left[\begin{array}{cc}
\underset{1}{\psi_{1}^{\mathrm{T}}} & \mathbf{0} \\
\underset{\sim}{0} & \underset{\sim}{\Psi_{1}^{\mathrm{T}}}
\end{array}\right]
\end{gathered}
$$




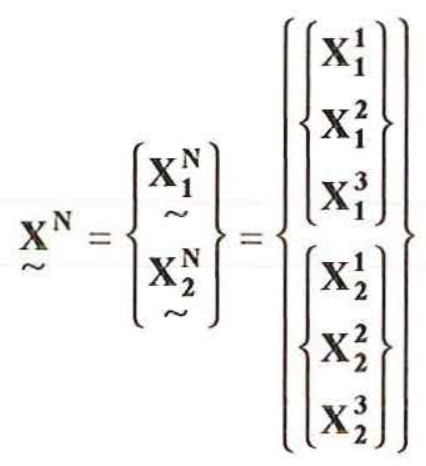

com

$$
\underset{\sim}{\Psi_{1}}=\left\{\begin{array}{l}
\xi_{1}^{\mathrm{P}} \\
\xi_{2}^{\mathrm{P}} \\
\xi_{3}^{\mathrm{P}}
\end{array}\right\}
$$

A coordenada $\mathbf{x}_{\mathbf{i}}$ do ponto " $p$ " pode ser escrita explicitamente como:

$$
\mathbf{X}_{\mathbf{i}}^{\mathrm{P}}=\xi_{1}^{\mathrm{P}} \cdot \mathbf{X}_{\mathbf{i}}^{1}+\xi_{2}^{\mathrm{P}} \cdot \mathbf{X}_{\mathbf{i}}^{2}+\xi_{3}^{\mathrm{P}} \cdot \mathbf{X}_{\mathbf{i}}^{3}
$$

A variável adimensional $\xi_{\alpha}^{\mathbf{P}}$ em função das coordenadas $\mathbf{x}_{\mathbf{i}}^{\mathbf{P}}$ do ponto " $\mathbf{p}$ " fica:

$$
\xi_{\alpha}^{\mathrm{P}}=\frac{1}{2 \cdot \mathrm{A}} \cdot\left(2 \cdot \mathrm{A}_{0}^{\alpha}+b^{\alpha} \cdot \mathrm{x}_{1}^{\mathrm{P}}+\mathrm{a}^{\alpha} \cdot \mathrm{x}_{2}^{\mathrm{P}}\right)
$$

onde:

$$
\begin{aligned}
& a^{\alpha}=X_{1}^{k}-X_{1}^{j} \\
& b^{\alpha}=X_{2}^{j}-X_{2}^{k} \\
& 2 \cdot A_{0}^{\alpha}=X_{1}^{j} \cdot X_{2}^{k}-X_{1}^{k} \cdot X_{2}^{j} \\
& A=\frac{1}{2} \cdot\left(b^{1} \cdot a^{2}-b^{2} \cdot a^{1}\right)
\end{aligned}
$$

para $\quad \alpha=1,2,3 \quad j=2,3,1 \quad k=3,1,2$ 
Como as integrais envolvem soluções fundamentais, as quais, por sua vez, estão expressas no sistema de coordenadas polares, é conveniente expressar a equação (4.42) em coordenadas polares $(\mathbf{r}, \theta)$, com origem no ponto “ “q", figura 4.20:

$$
\xi_{\alpha}^{\mathrm{P}}=\xi_{\alpha}^{\mathrm{q}}+\frac{\mathbf{r}}{2 \cdot \mathbf{A}} \cdot\left(\mathbf{b}^{\alpha} \cdot \cos (\theta)+\mathbf{a}^{\alpha} \cdot \operatorname{sen}(\theta)\right)
$$

onde $\xi_{\alpha}^{q}$ é obtido através da equação (4.41) para o ponto “q".

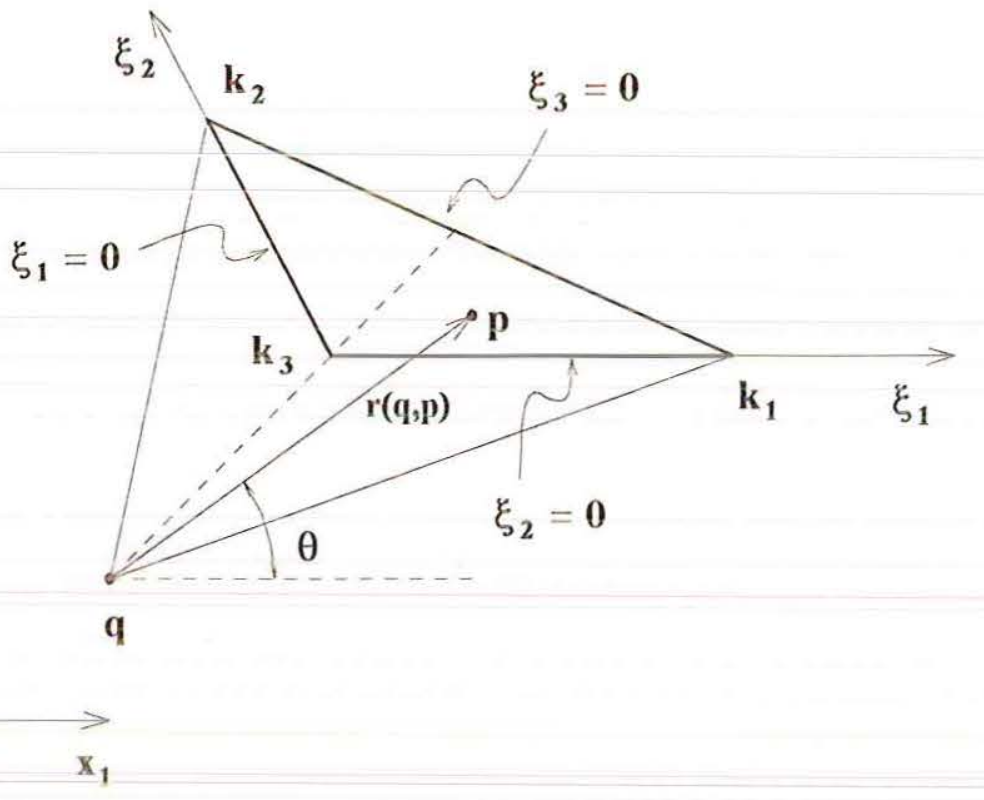

FIGURA 4.20 - Célula Interna e os Sistemas de Coordenadas $\left(\mathrm{x}_{1}, \mathrm{x}_{2}\right)$, $\left(\xi_{1}, \xi_{2}\right)$ e $(r, \theta)$.

De forma análoga à expressão (4.35), pode-se escrever o deslocamento transversal $w(\mathbf{p})$ de um ponto qualquer da célula em função dos deslocamentos verticais dos vértices da mesma, figura 4.16, isto é:

$$
w(\mathrm{p})=\underset{\sim}{\Psi} \underset{1}{\mathrm{~T}} \cdot{\underset{\sim}{w}}^{N}
$$


onde $\psi_{1}$ é dado em (4.40) e $w^{N}$, representa os deslocamentos verticais $w^{1}, w^{2}, w^{3}$ nos vértices da célula.

Substituindo-se em (4.34) o valor de $w(p)$ dado em (4.45), considerando-se apenas uma célula genérica "i", e escrevendo-se $\Omega_{\mathbf{i}}=\Omega_{\mathrm{gsi}}$, por simplificação, obtém-se:

$$
\int_{\Omega_{i}} \bar{K}^{*}(\mathbf{q}, \mathbf{p}) \cdot w(\mathbf{p}) \cdot \mathrm{d} \Omega_{\mathrm{i}}(\mathrm{p})
$$

$\left.=\left\{\int_{\Omega_{i}} \xi_{1}^{P} \cdot \bar{K}^{*}(q, p) \cdot d \Omega_{i}(p) \int_{\Omega_{i}} \xi_{2}^{P} \cdot \bar{K}^{*}(q, p) \cdot d \Omega_{i}(p) \int_{\Omega_{i}} \xi_{3}{ }^{p} \cdot \bar{K}^{*}(q, p) \cdot d \Omega_{i}(p)\right)\right\} \cdot\left\{\begin{array}{l}w^{1} \\ w^{2} \\ w^{3}\end{array}\right\}$

ou:

$$
\int_{\Omega_{i}} \overline{\mathbf{K}}^{*}(\mathbf{q}, \mathbf{p}) \cdot w(\mathbf{p}) \cdot \mathrm{d} \Omega_{\mathrm{i}}(\mathbf{p})=\left\{\begin{array}{lll}
\mathrm{A}^{1} & \mathrm{~A}^{2} & \mathrm{~A}^{3}
\end{array}\right\} \cdot\left\{\begin{array}{l}
w^{1} \\
w^{2} \\
w^{3}
\end{array}\right\}
$$

onde:

$$
A^{\alpha}=\int_{\Omega_{i}} \xi_{\alpha}^{p} \cdot \bar{K}^{*}(q, p) \cdot d \Omega_{i}(p) \quad \alpha=1,2,3
$$

Considerando-se variação linear da rigidez pode-se escrever,

$$
\mathrm{D}\left(\mathrm{x}_{1}, \mathrm{x}_{2}\right)=\mathrm{FD} \cdot \mathrm{x}_{1}+\mathrm{GD} \cdot \mathrm{x}_{2}+\mathrm{HD}
$$

Assim suas derivadas ficam,

$$
\frac{\partial \mathrm{D}\left(\mathrm{x}_{1}, \mathrm{x}_{2}\right)}{\partial \mathrm{x}_{1}}=\mathbf{F D}
$$




$$
\begin{aligned}
& \frac{\partial \mathbf{D}\left(\mathbf{x}_{1}, \mathbf{x}_{2}\right)}{\partial \mathbf{x}_{2}}=\mathbf{G D} \\
& \frac{\partial^{2} \mathbf{D}\left(\mathbf{x}_{1}, \mathbf{x}_{2}\right)}{\partial \mathbf{x}_{1} . \partial \mathbf{x}_{2}}=\mathbf{0}
\end{aligned}
$$

Admitindo-se que:

$$
q_{i}^{*}=\frac{-r, i_{i}}{4 . \pi . r}
$$

pode-se obter o valor de $\overline{\mathbf{K}}^{*}(\mathbf{q}, \mathbf{p})$ para o caso de rigidez com variação linear, isto é:

$$
\bar{K}^{*}(q, p)=\frac{-2}{D_{0}} \cdot\left[\text { FD. } \frac{r_{1}}{4 \cdot \pi \cdot r}+\text { GD. } \frac{r_{2}}{4 \cdot \pi \cdot r}\right]
$$

Substituindo-se o valor de $\overline{\mathbf{K}}^{*}(\mathbf{q}, \mathbf{p})$ na integral $(4.46)$, a integral de domínio resulta:

$$
\frac{-2}{D 0.4 \cdot \pi} \int_{\Omega}\left(\frac{F D \cdot r_{91}+\text { GD.r. },_{2}}{r}\right) \cdot w(p) \cdot d \Omega
$$

Transformando-se em coordenadas polares a integral de domínio resulta:

$$
\frac{-2}{D 0.4 \cdot \pi} \int_{\theta \mathrm{r}}\left(\mathrm{FD}, \mathrm{r}_{1}+\mathrm{GD} \cdot \mathrm{r}, 2\right) \cdot w(\mathrm{p}) \cdot \mathrm{dr} \cdot \mathrm{d} \theta
$$

Substituindo-se na integral (4.53) a expressâo de $w(\mathrm{p})$ dada pela equação (4.44) e transformando-se $\xi_{\alpha}^{P}$ para coordenadas polares, a equação (4.53) fica:

$$
\frac{-2}{D 0.4 \cdot \pi} \iint_{0}\left(\text { FD. } r,_{1}+\text { GD. } r,{ }_{2}\right) \cdot\left[\xi_{\alpha}^{q}+\frac{r}{2 \cdot A} \cdot\left(b^{\alpha} \cdot \cos (\theta)+a^{\alpha} \cdot \operatorname{sen}(\theta)\right)\right] \cdot d r \cdot d \theta
$$

Integrando-se (4.54) em r, resulta: 


$$
\begin{aligned}
& \frac{-2}{\text { Do.4. } \pi} \int_{\theta} r \cdot \xi_{\alpha}^{q} \cdot\left(\text { FD. },,_{1}+\text { GD. } r,,_{2}\right)+\mathbf{r}^{2} \cdot\left(\mathbf{b}^{\alpha} \cdot \cos (\theta)+\mathbf{a}^{\alpha} \cdot \operatorname{sen}(\theta)\right) \\
& \frac{\left(\text { FD. } r,{ }_{1}+\text { GD. } r,{ }_{2}\right)}{4 . A} \cdot d \theta
\end{aligned}
$$

Substituindo-se $\mathbf{d} \theta=\frac{\mathbf{1}}{\mathbf{r}} \cdot \frac{\partial \mathbf{r}}{\partial \mathbf{n}} \cdot \mathbf{d} \Gamma=\frac{\mathbf{r}_{\mathbf{i}} \cdot \mathbf{n}_{\mathbf{i}}}{\mathbf{r}} \cdot \mathbf{d} \Gamma$ na eq.(4.55), resulta em:

$$
\left[\frac{-2}{\operatorname{Do.4.\pi }} \int_{\Gamma_{\mathrm{c}}}\left[\xi_{\alpha}^{\mathrm{q}}+\frac{\mathbf{r} \cdot\left(\mathbf{b}^{\alpha} \cdot \cos (\theta)+\mathrm{a}^{\alpha} \cdot \operatorname{sen}(\theta)\right)}{4 \cdot \mathrm{A}}\right] \cdot\left(\mathrm{FD} \cdot \mathrm{r}_{,_{1}}+\mathrm{GD} \cdot \mathrm{r}_{2}\right) \cdot \frac{\partial \mathbf{r}}{\partial \mathbf{n}} \cdot \mathrm{d} \Gamma_{\mathrm{c}}\right] \cdot w(\alpha)
$$

Transforma-se, assim, a integral de domínio (4.47) de uma célula em uma integral de linha sobre o contorno da célula.

Considerando-se que a célula contém três lados a integral (4.56) pode ser rescrita como:

$$
\left[\frac{-2}{D 0.4 \cdot \pi} \cdot \sum_{\mathrm{i}=1}^{3}\left(\int_{\Gamma_{\mathrm{ci}}}\left[\xi_{\alpha}^{q}+\frac{\mathrm{r} \cdot\left(\mathrm{b}^{\alpha} \cdot \cos (\theta)+\mathrm{a}^{\alpha} \cdot \operatorname{sen}(\theta)\right)}{4 \cdot \mathrm{A}}\right] \cdot\left(\mathrm{FD} \cdot \mathrm{r}_{1_{1}}+\mathrm{GD} \cdot \mathrm{r}_{2}\right) \cdot \frac{\partial \mathrm{r}}{\partial \mathrm{n}} \cdot \mathbf{d \Gamma _ { \mathrm { ci } }}\right)\right] \cdot w(\alpha)
$$

para $\alpha=1,2,3$.

\subsection{Definição das Posições do Ponto de Colocação}

No problema de placas de Kirchhoff com espessura constante têm-se quatro variáveis envolvidas no contorno da placa, que são: $\mathbf{V}_{\mathbf{n}}, \mathbf{M}_{\mathbf{n}}, w$ e $\frac{\partial w}{\partial \mathbf{n}}$ e devido a existência dos cantos têm-se mais duas variáveis envolvidas $\mathbf{R}_{\mathbf{c}}$ e $w_{\mathbf{c}}$ associadas apenas a esses pontos particulares. 
Para cada ponto do contorno duas das variáveis devem ser conhecidas e duas são incógnitas. Já no canto uma deve ser conhecida e outra incógnita.

Para o problema de placas de Kirchhoff com espessura variável, além das variáveis discutidas acima, surgirão mais duas variáreis envolvidas $\mathbf{R}_{\mathbf{i}}$ e $\boldsymbol{w}_{\mathbf{i}}$ onde uma deverá ser incógnita e outra prescrita, devido a existência de pontos no interior da placa. Com isso infinitas alternativas podem definir o sistema de equação integral dos deslocamentos. Dentre estas alternativas pode-se citar:

- Utilizando as equações integrais de $w$ e $\frac{\partial w}{\partial \mathbf{n}}$.

Neste caso para cada ponto do contorno define as equações integrais de deslocamento transversal e rotação podendo ainda assumir as seguintes configurações:

- $\mathbf{R}_{\mathbf{c}}$ e $w_{\mathbf{c}}$ são tratados como contribuição dos nós vizinhos em cada canto;

- $\mathbf{R}_{\mathbf{c}}$ e $w_{\mathbf{c}}$ são tratados como variáveis do problema.

- Utilizando apenas a equação integral de $w$.

Neste caso para cada ponto do contorno terá dois ponto de carga onde serão definidas as equações integrais de deslocamento transversal, podendo ainda assumir as seguintes configurações:

- $\mathbf{R}_{\mathrm{c}}$ e $w_{\mathrm{c}}$ são tratados como contribuição dos nós vizinhos em cada canto;

- $\mathbb{R}_{\mathrm{c}}$ e $w_{\mathrm{c}}$ sấo considerados como variáveis do problema.

As possibilidades do tratamento da montagem do sistema de equações citadas acima estão descritas em TEJERINA CALDERÓN (1991).

Neste trabalho optou-se por usar apenas a equação integral de deslocamento transversal para manter todas as equações de mesma natureza. Por utilizar apenas a equação integral de deslocamentos, tem-se a necessidade também de expressar a equação integral de deslocamento transversal em um outro ponto que não seja incógnita do problema, podendo este ponto ser um ponto externo à placa $(\mathrm{A})$, figura 4.21 . 
As variáveis de canto $\mathbf{R}_{\mathbf{c}}$ e $w_{\mathrm{c}}$ serão consideradas como variáveis do problema. Devido a isso, serão escritas equações integrais de deslocamentos também para os cantos da placa, figura 4.21 .

Para os pontos internos também serão escritas equações integrais de deslocamentos. Logo pontos de colocação serão definidas nas seguintes posições:

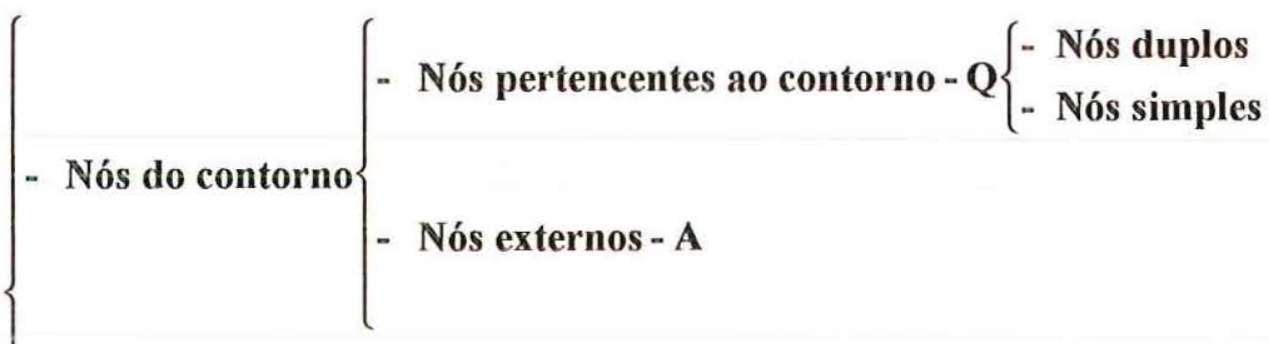

- Nós de canto

- Nós internos

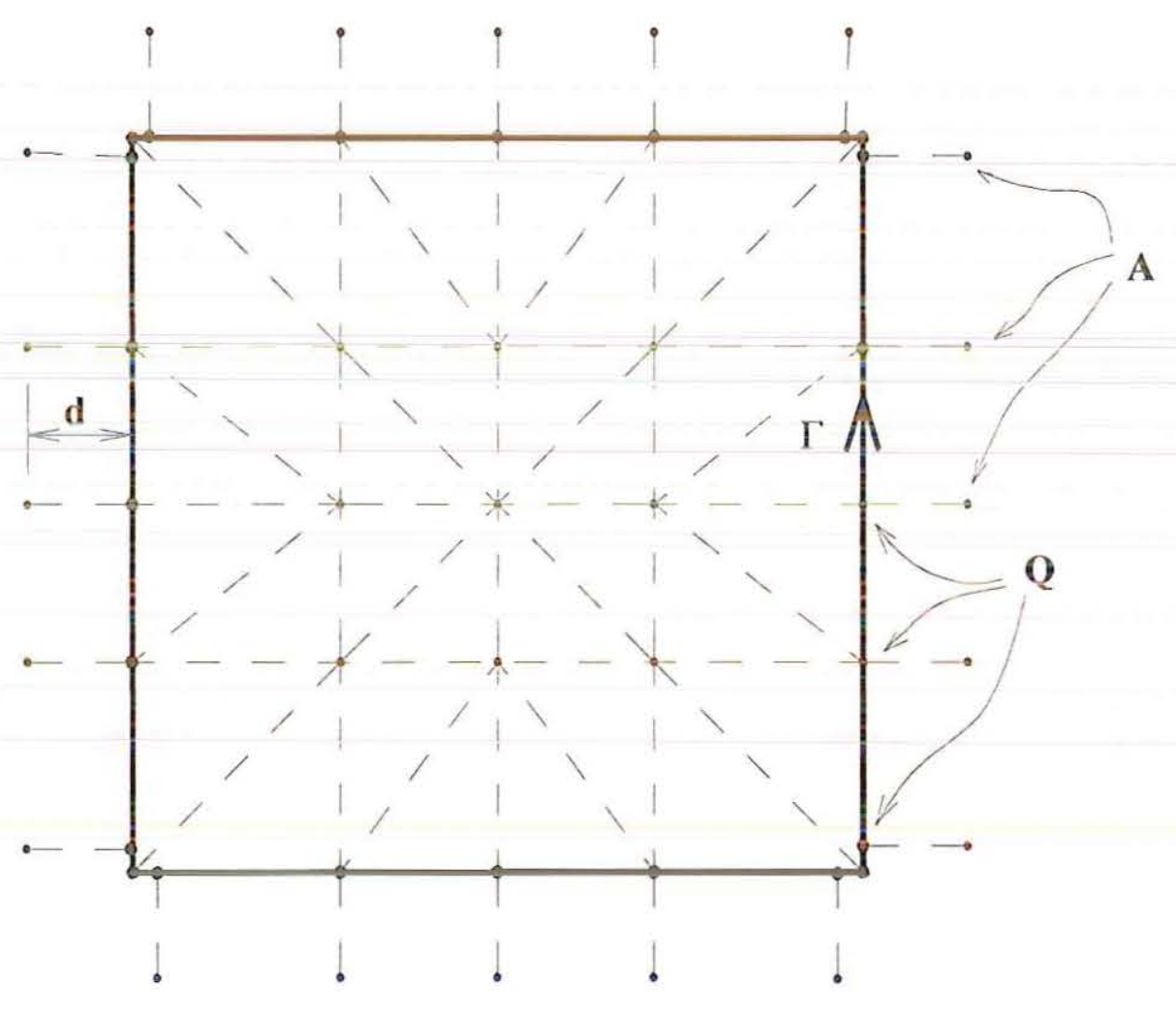

FIGURA 4.21 - Posições do Ponto de Colocação. 
A posição do ponto "A" é definido na direção normal ao elemento, a uma distância do nó, figura 4.22, dada por:

$$
\mathbf{d}=\mathbf{a} \cdot \ell_{\mathbf{m}}
$$

onde:

a - coeficiente maior que zero, cujo valor pode estar situado convenientemente entre 0,5 e 1,50, SILVA (1996).

$\ell_{\mathrm{m}}$ - média dos comprimentos dos elementos concorrentes no nó, ou simplesmente o comprimento do elemento, no caso de nó duplo.

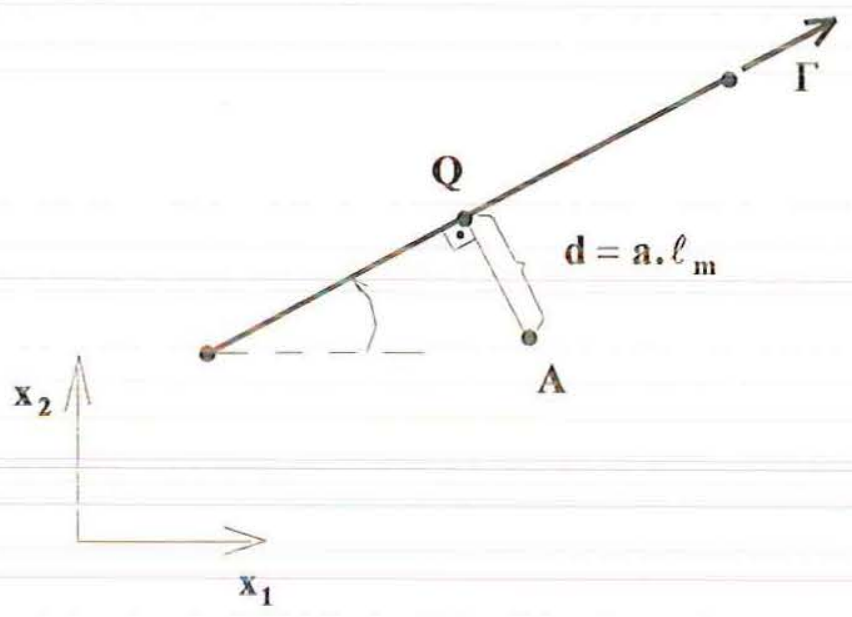

FIGURA 4.22 - Coeficiente de Posicionamento a.

Quando o ponto de colocação é um nó duplo este deverá sofrer um recuo para o interior do elemento para eliminar a singularidade, como já foi visto anteriormente, no item 4.3.8. Este recuo do ponto de colocação deve ser adotado entre 0,4 e 0,6 em termos de coordenadas adimensional em módulo, figura 4.23. 


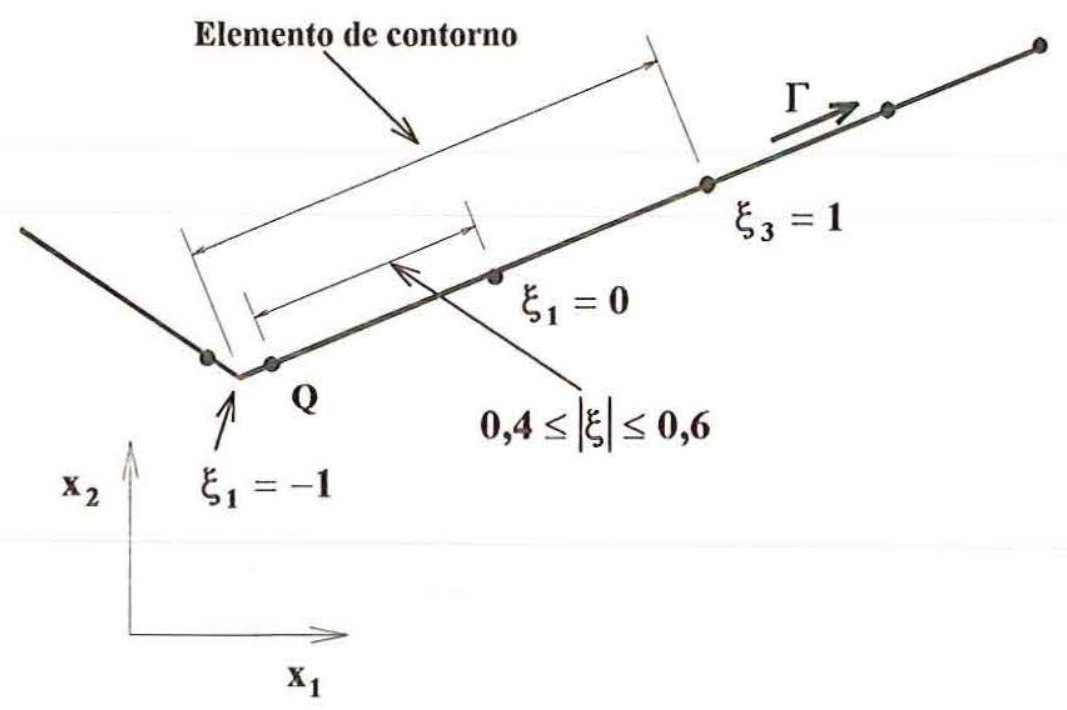

FIGURA 4.23 - Recuo do Nó Dupio.

\subsection{Transformação das Equações Integrais em Equações Algébricas}

A equação integral de deslocamentos de um ponto $\mathbf{Q}$ qualquer do contorno, dado em (3.64), pode ser escrita em função dos deslocamentos $\underset{\sim}{\mathbf{u}}$, esforços $\underset{\sim}{\mathbf{p}}$ e dos respectivos vaiores fundamentais, na seguinte forma:

$$
\begin{aligned}
& \overline{\mathbf{C}}(\mathrm{Q}) \cdot \mathrm{u}(\mathrm{Q})+\int_{\Gamma} \overline{\mathrm{p}}_{\sim}^{*}(\mathrm{Q}, \mathrm{P}) \cdot \underset{\sim}{\mathrm{u}}(\mathrm{P}) \cdot \mathrm{d} \Gamma(\mathrm{P})+\sum_{\mathrm{i}=\mathbf{1}}^{\mathrm{Ne}} \overline{\mathbf{p}}_{\mathrm{ci}}^{*}(\mathrm{Q}, \mathrm{P}) \cdot w_{\mathrm{ci}}(\mathrm{P})
\end{aligned}
$$

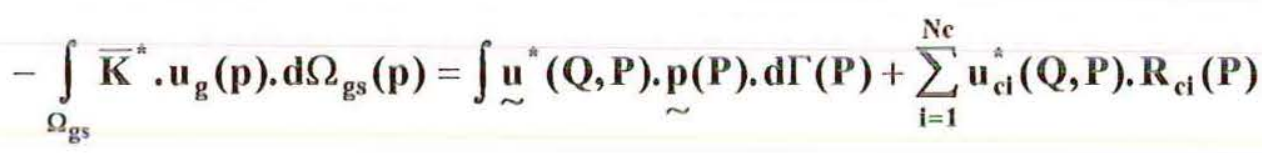

$$
\begin{aligned}
& +\int_{\Omega_{g}} g(p) \cdot u_{g}^{*}(Q, P) \cdot d \Omega_{g}(p)
\end{aligned}
$$


onde:

$$
\begin{aligned}
& \overline{\mathbf{C}}(\mathbf{Q})=\mathbf{C}(\mathbf{Q}) \cdot \frac{\mathbf{D}(\mathbf{Q})}{\mathbf{D}_{\mathrm{o}}} \\
& \underset{\sim}{\mathbf{u}(P)}=\left\{\begin{array}{l}
\mathbf{u}_{1}(\mathbf{P}) \\
\mathbf{u}_{2}(\mathbf{P})
\end{array}\right\}=\left\{\begin{array}{l}
w(P) \\
\frac{\partial w}{\partial \mathbf{n}}(\mathbf{P})
\end{array}\right\} \\
& \underset{\sim}{\mathbf{p}(P)}=\left\{\begin{array}{l}
\mathbf{p}_{1}(\mathbf{P}) \\
\mathbf{p}_{2}(\mathbf{P})
\end{array}\right\}=\left\{\begin{array}{l}
\mathbf{V}_{\mathrm{n}}(\mathbf{P}) \\
\mathbf{M}_{\mathrm{n}}(\mathbf{P})
\end{array}\right\}
\end{aligned}
$$

$$
\stackrel{\sim}{\mathbf{p}}^{*}=\left\{\left[\frac{1}{D_{0}} \cdot\left(\mathbf{D}(\mathrm{s}) \cdot \mathbf{V}_{\mathrm{n}}^{*}(\mathbf{Q}, \mathbf{P})+2 \cdot \frac{\partial \mathrm{D}(\mathrm{s})}{\partial \mathrm{s}} \cdot \mathbf{M}_{\mathrm{ns}}^{*}(\mathbf{Q}, \mathbf{P})+\frac{\partial \mathrm{D}(\mathrm{s})}{\partial \mathrm{n}} \cdot \mathbf{M}_{\mathrm{n}}^{*}(\mathbf{Q}, \mathbf{P})\right)\right]\right.
$$

$$
\left.\left[-\frac{D(s)}{D_{0}} \cdot M_{n}^{*}(Q, P)\right]\right\}
$$

$$
u^{*}(Q, P)=\left[w^{*}(Q, P) \quad-\frac{\partial w^{*}}{\partial \mathrm{n}}(\mathrm{Q}, \mathrm{P})\right]
$$

$$
\bar{K}^{*}(q, p)=\frac{1}{D 0} \cdot\left[2 \cdot \frac{\partial D(s)}{\partial x_{i}} \cdot q_{i}^{*}(Q, p)+\frac{\partial^{2} D(s)}{\partial x_{i} \cdot \partial x_{j}} \cdot m_{i j}^{*}(Q, p)\right]
$$

$$
u_{\mathrm{g}}(\mathrm{p})=w(\mathrm{p})
$$

$$
\mathrm{u}_{\mathrm{g}}^{*}(\mathrm{Q}, \mathrm{p})=w^{*}(\mathbf{Q}, \mathbf{p})
$$

$$
\mathbf{u}(\mathbf{Q})=w(\mathbf{Q})
$$




$$
\begin{aligned}
& \overline{\mathbf{p}}_{\mathrm{ci}}^{*}(\mathbf{Q}, \mathbf{P})=\mathbf{R}_{\mathrm{ci}}^{*} \cdot \frac{D_{\mathrm{ci}}}{D_{\mathrm{o}}} \\
& \mathrm{u}_{\mathrm{ci}}^{*}(\mathrm{Q}, \mathrm{P})=w_{\mathrm{ci}}^{*} \cdot \frac{\mathrm{D}_{\mathrm{ci}}}{D_{\mathrm{o}}}
\end{aligned}
$$

Considerando-se o contorno discretizado por "Ne" elementos de contorno e discretizando-se o domínio em células como feito no item 4.4.2, e substituindo-se as variáveis por suas aproximações (4.8.a), (4.8.b) e (4.45), a equação (4.59) fica:

$$
\begin{aligned}
& \overline{\mathbf{C}}(\mathbf{Q}) \cdot \mathrm{u}(\mathbf{Q})+\sum_{\mathrm{j}=1}^{\mathrm{Ne}} \int_{\Gamma_{\mathrm{j}}}^{\bar{p}_{\sim}^{*}}(\mathbf{Q}, \mathbf{P}) \cdot \underset{\sim}{\phi}(\mathbf{P}) \cdot \mathrm{d} \Gamma(\mathbf{P}) \cdot \underset{\sim}{\mathrm{U}_{j}^{N}}(\mathbf{P})+\sum_{\mathrm{i}=1}^{\mathrm{Nc}} \overline{\mathbf{p}}_{\mathrm{ci}}^{k}(\mathbf{Q}, \mathbf{P}) \cdot w_{\mathrm{ci}}(\mathrm{P})
\end{aligned}
$$

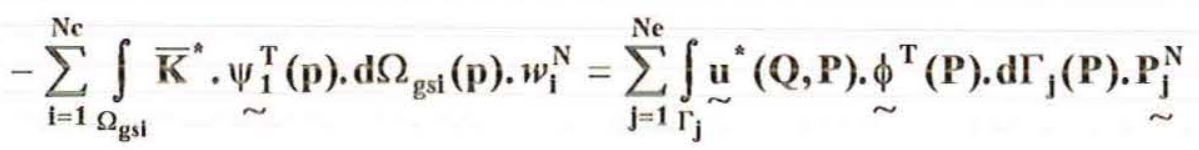

$$
\begin{aligned}
& +\sum_{i=1}^{N c} u_{c i}^{*}(Q, P) \cdot R_{c i}(P)+\int_{\Omega_{g}} g(p) \cdot u_{g}^{*}(Q, P) \cdot d \Omega_{g}(p)
\end{aligned}
$$

Assim, as integrais de coniomo que aparecem na eq.(4.71) têm valores conhecidos, portanto estas integrais podem ser calculadas para cada elemento $\Gamma_{\mathrm{j}} \mathrm{e}$ são representadas, genericamente por:

$$
\begin{aligned}
& \underset{\sim}{\bar{h}^{\mathrm{j}}}(\mathrm{Q})=\int_{\Gamma_{\mathrm{j}}} \overline{\mathrm{p}}^{\mathrm{*}}(\mathrm{Q}, \mathbf{P}) \cdot \underset{\sim}{\phi^{\mathrm{T}}}(\mathrm{P}) \cdot \mathrm{d} \Gamma_{\mathrm{j}}(\mathrm{P}) \\
& \underset{\sim}{g}(Q)=\int_{\Gamma_{j}} \underset{\sim}{u^{*}}(Q, P) \cdot \underset{\sim}{\phi}{ }^{T}(P), d \Gamma_{j}(P)
\end{aligned}
$$

A integral de domínio, correspondente ao carregamento transversal, também é conhecida. Sua obtenção foi feita no item 4.4.1. Após sua integração é conveniente representá-la por: 


$$
t(Q)=\int_{\Omega_{g}} g(p) \cdot u_{g}^{*}(Q, P) \cdot d \Omega_{g}(p)
$$

A integral de domínio, correspondente ao deslocamento, pode ser expressa em função dos parâmetros nodais feito no item 4.4.2, após integração obtém-se:

$$
\underset{\sim}{s^{\ell}}(Q)=-\int_{\Omega_{\mathrm{gsi}}} \overline{\mathrm{K}}^{*} \cdot \underset{\sim}{\Psi_{1}^{\mathrm{T}}}(\mathrm{p}) \cdot \mathrm{d} \Omega_{\mathrm{gsi}}(\mathrm{p})
$$

Assim, a equação (4.71) pode ser escrita da seguinte forma:

$$
\begin{aligned}
& \overline{\mathrm{C}}(\mathrm{Q}) \cdot \mathrm{u}(\mathrm{Q})+\sum_{\mathrm{j}=1}^{\mathrm{Ne}} \underset{\sim}{\overline{\mathbf{h}}^{\mathrm{j}}} \cdot{\underset{\sim}{\mathrm{j}}}_{\mathrm{j}}^{\mathrm{N}}(\mathrm{P})+\sum_{\mathrm{i}=1}^{\mathrm{Ne}} \overline{\mathrm{p}}_{\mathrm{ci}}^{\mathrm{N}}(\mathrm{Q}, \mathrm{P}) \cdot w_{\mathrm{ci}}(\mathrm{P})
\end{aligned}
$$

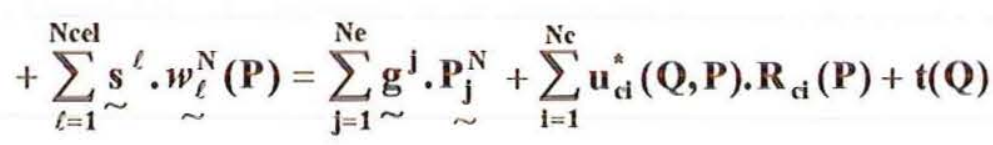

O deslocamento do ponto de colocação (que não é valor de contorno escolhido para o problema discretizado) devido a influência dos "Ne" elementos de contomo, dos "Ncel" células e dos "Ne" cantos da placa, a eq.(4.76) pode ser escrita como:

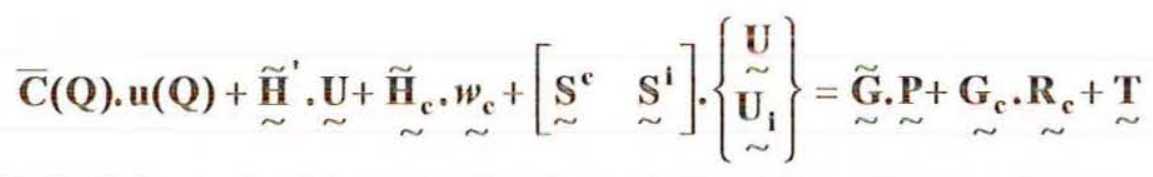

sendo:

- $\bar{C}(\mathbf{Q}) \cdot \mathbf{u}(\mathbf{Q})=\frac{1}{2} \cdot \frac{\mathrm{D}(\mathbf{Q})}{\mathrm{D}_{\mathrm{o}}} \cdot \mathbf{u}(\mathbf{Q})$, quando o ponto de carga está no contorno(Q);

- $\overline{\mathbf{C}}(\mathbf{Q}) \cdot \mathbf{u}(\mathbf{Q})=\mathbf{0}$, quando o ponto de carga está no exterior $(\mathbf{A})$; 
Quando o ponto de colocação estiver no contorno o deslocamento $\mathbf{u}(\mathbf{Q})$ pode ser escrito em função dos deslocamentos $\underset{\sim}{\mathbf{U}_{\mathbf{b}}^{\mathbf{j}}}$ do elemento ao qual pertence este ponto $\mathbf{Q}$, figura 4.24. Utilizando para isso as funções de forma, resultando em:

$$
\frac{\mathbf{D}(\mathbf{Q})}{\mathbf{D}_{0}} \cdot \mathbf{C}(\mathbf{Q}) \cdot \mathbf{u}(\mathbf{Q})=\frac{\mathbf{D}(\mathbf{Q})}{\mathbf{D}_{0}} \cdot \frac{1}{2} \cdot\left[\begin{array}{lll}
\phi_{1} & \phi_{2} & \phi_{3}
\end{array}\right] \cdot\left\{\begin{array}{l}
\mathbf{U}^{\mathrm{n} 1} \\
\mathbf{U}^{\mathrm{n} 2} \\
\mathbf{U}^{\mathrm{n} 3}
\end{array}\right\}
$$

Desta forma, os coeficientes oriundos destes deslocamentos podem ser incorporados ao vetor $\underset{\sim}{\tilde{\mathbf{H}}}$, resultando em:

$$
\left[\underset{\sim}{\tilde{\mathbf{H}}}+\underset{\sim}{\mathbf{S}^{\mathbf{c}}}\right] \cdot \underset{\sim}{\mathbf{U}_{\mathbf{b}}}+\underset{\sim}{\tilde{\mathbf{H}}_{\mathbf{c}}} \cdot w_{\sim}+\underset{\sim}{\mathbf{S}^{\mathbf{i}}} \cdot \underset{\sim}{\mathbf{U}_{\mathbf{i}}}=\underset{\sim}{\widetilde{\mathbf{G}}} \cdot \underset{\sim}{\mathbf{P}_{\mathbf{b}}}+\underset{\sim}{\mathbf{G}_{\mathbf{c}}} \cdot \mathbf{R}_{\mathbf{c}}+\underset{\sim}{\mathbf{T}}
$$

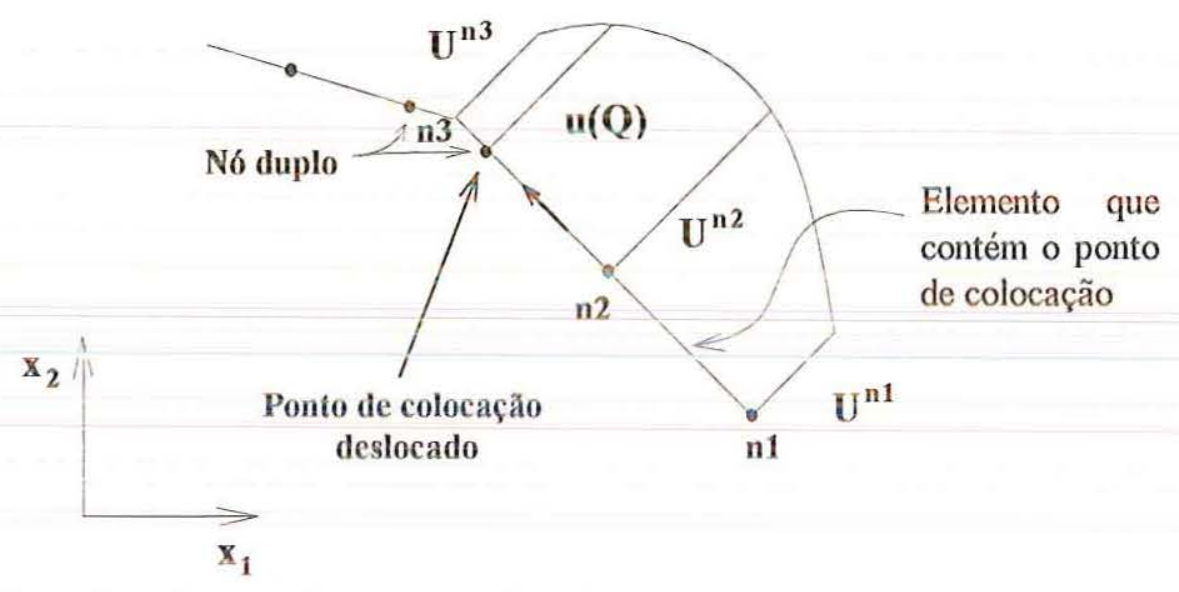

FIGURA 4.24 - Função Quadrática no Elemento de Integração

Devido a influência dos "Ne" elementos de contorno, dos "Ncel" células e dos "Nc" cantos da placa, sobre o ponto de colocação quando este está sobre o canto, a eq.(4.75) pode ser escrita como: 


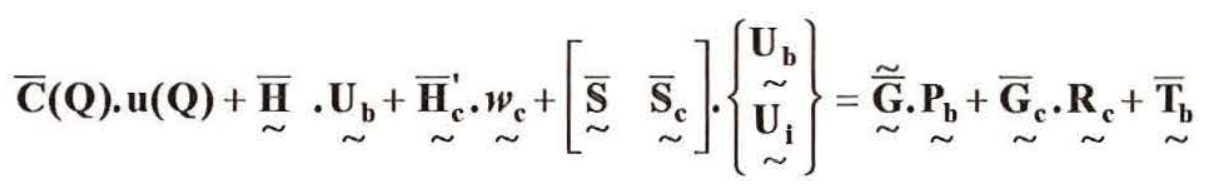

sendo:

- $\bar{C}(Q) \cdot u(Q)=\frac{\beta_{c}}{2 . \pi} \cdot \frac{D_{\mathrm{ci}}(Q)}{D_{0}} \cdot w_{c}(Q)$

Os coeficientes oriundos dos cantos podem ser incorporados ao vetor $\overline{\mathbf{H}}_{\mathbf{c}}^{\prime}$, resultando em:

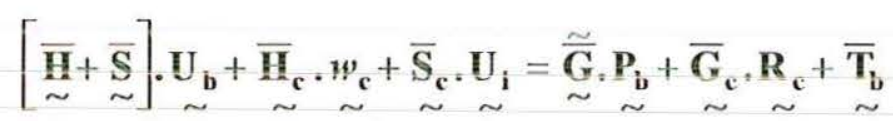

Analogamente a obtenção de (4.76) e (4.79), quando o ponto de colocação for definido no interior, a eq.(4.75) fica:

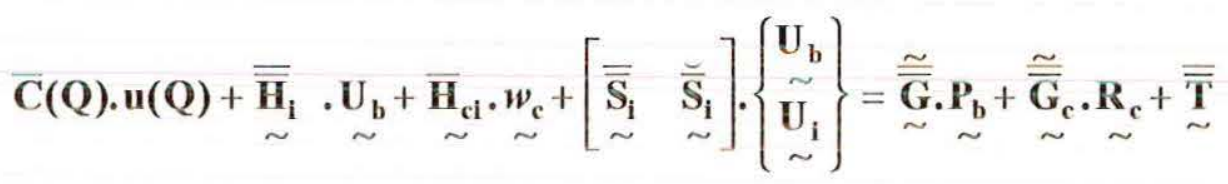

sendio:

- $\bar{C}(Q) \cdot u(Q)=\frac{D(Q)}{D_{0}} \cdot U_{1}$

Os coeficientes oriundos dos deslocamentos dos pontos internos podem ser incorporados ao vetor $\underset{\sim}{\breve{S}_{\mathbf{c}}}$, resultando:

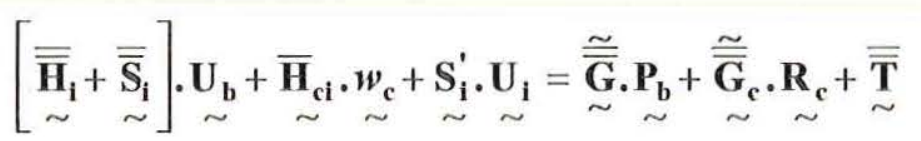

Agrupando-se (4.79), (4.82) e (4.85), resulta: 


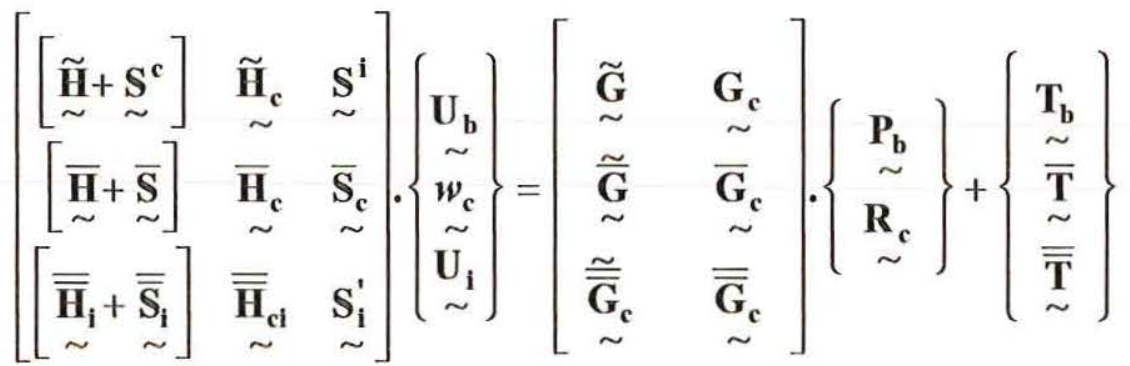

Sendo:

$\underset{\sim}{\mathbf{U}^{\mathrm{T}}}=\left\{\begin{array}{llllllll}w^{1} & \frac{\partial w^{1}}{\partial n} & w^{2} & \frac{\partial w^{2}}{\partial n} & \ldots & \ldots & w^{\mathrm{Nn}} & \frac{\partial w^{\mathrm{Nn}}}{\partial n}\end{array}\right\}$

$\underset{\sim}{\mathbf{P}^{T}}=\left\{\begin{array}{llllllll}\mathbf{V}_{n}^{1} & \mathbf{M}_{n}^{1} & \mathbf{V}_{n}^{2} & \mathbf{M}_{n}^{2} & \ldots & \ldots & \mathbf{V}_{n}^{N n} & \mathbf{M}_{n}^{N n}\end{array}\right\}$

$\underset{\sim}{w_{\mathrm{c}}^{\mathrm{T}}}=\left\{\begin{array}{lllll}w_{\mathrm{c} 1} & w_{\mathrm{c} 2} & w_{\mathrm{c} 3} & \ldots & w_{\mathrm{cNc}}\end{array}\right\}$

$\underset{\sim}{\mathbb{R}_{c}^{\mathrm{T}}}=\left\{\begin{array}{lllll}\mathbf{R}_{\mathrm{c} 1} & \mathbf{R}_{\mathrm{c} 2} & \mathbf{R}_{\mathrm{c} 3} & \ldots & \mathbf{R}_{\mathrm{cNc}}\end{array}\right\}$

$$
\underset{\sim}{\mathrm{U}_{\mathrm{i}}}=\left\{\begin{array}{lllll}
w_{\mathrm{i} 1} & w_{\mathrm{i} 2} & w_{\mathrm{i} 3} & \ldots & w_{\mathrm{iNcel}}
\end{array}\right\}
$$

Pode-se, ainda escrever (4.86) como:

$$
\left[\begin{array}{ccc}
\mathbf{H}_{\mathrm{b}} & \mathbf{H}_{\mathrm{c}} & \mathbf{H}_{\mathbf{i}}
\end{array}\right] \cdot\left\{\begin{array}{c}
\mathbf{U}_{\mathrm{b}} \\
\underset{\sim}{w_{\mathbf{c}}} \\
\underset{\sim}{\mathbf{U}_{\mathbf{i}}}
\end{array}\right\}=\left[\begin{array}{cc}
\mathbf{G}_{\mathrm{b}} & \mathbf{G}_{\mathrm{cc}} \\
\sim & \sim
\end{array}\right] \cdot\left\{\begin{array}{c}
\underset{\mathbf{P}}{\sim} \\
\underset{\mathbf{R}}{\mathbf{R}_{\mathbf{c}}} \\
\sim
\end{array}\right\}+\left\{\begin{array}{c}
\mathbf{T}_{\mathbf{b}} \\
\underset{\sim}{\mathbf{T}} \\
\underset{\sim}{\overline{\mathbf{T}}} \\
\sim
\end{array}\right\}
$$


ou ainda :

$$
\underset{\sim}{\mathbf{H}} \underset{\sim}{\mathbf{U}}=\underset{\sim}{\mathbf{G}} \cdot \underset{\sim}{\mathbf{P}}+\underset{\sim}{\mathbf{T}}
$$

O sistema de equações lineares (4.92.b), pode ser resolvido isolando-se todas as incógnitas no primeiro membro, isto é:

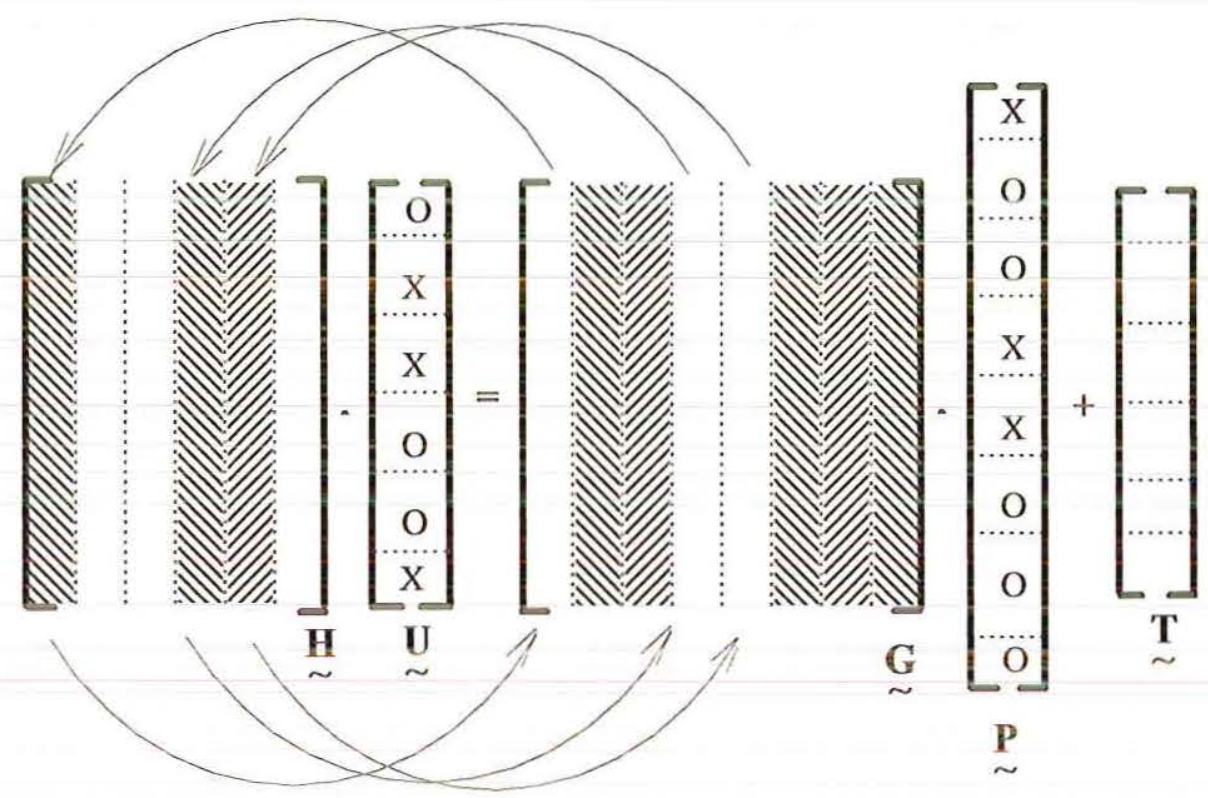

Obtendo-se assim, um sistema da forma:

$$
\underset{\sim}{\mathbf{A}} \underset{\sim}{\mathbf{X}}=\underset{\sim}{\mathbf{B}}
$$

onde:

X é o vetor das incógnitas, composto pelos deslocamentos e esforços nos nós, cantos e pontos internos da placa.

Resolvendo-se este sistema, obtêm-se todas as incógnitas da placa. 


\subsection{Propriedades da Matriz $\mathbf{H}$}

As propriedades da matriz $\underset{\sim}{\mathbf{H}}$ podem ser estabelecidas através da configuração de equilíbrio de uma placa. Se for admitido um carregamento transversal nulo, em uma placa com o contorno discretizado, os vetores dos esforços nodais $\underset{\sim}{\mathbf{P}} \mathrm{e}$ das integrais sobre a região carregada $\underset{\sim}{\mathbf{T}}$, no sistema de equações (4.92), são também nulos, isto é:

$$
\mathrm{H}_{\mathrm{b}} \cdot \underset{\sim}{\mathrm{U}}+\underline{\sim}_{\mathrm{c}} \cdot w_{\mathrm{c}}+\mathrm{H}_{\mathrm{i}} \cdot \mathrm{U}_{\mathrm{i}}=\mathbf{0}
$$

Este sistema de equação admite solução não triviais, correspondentes aos movimentos de corpo rígido, isto é, deslocamento vertical e rotação em torno de um eixo arbitrário, PAIVA (1987).

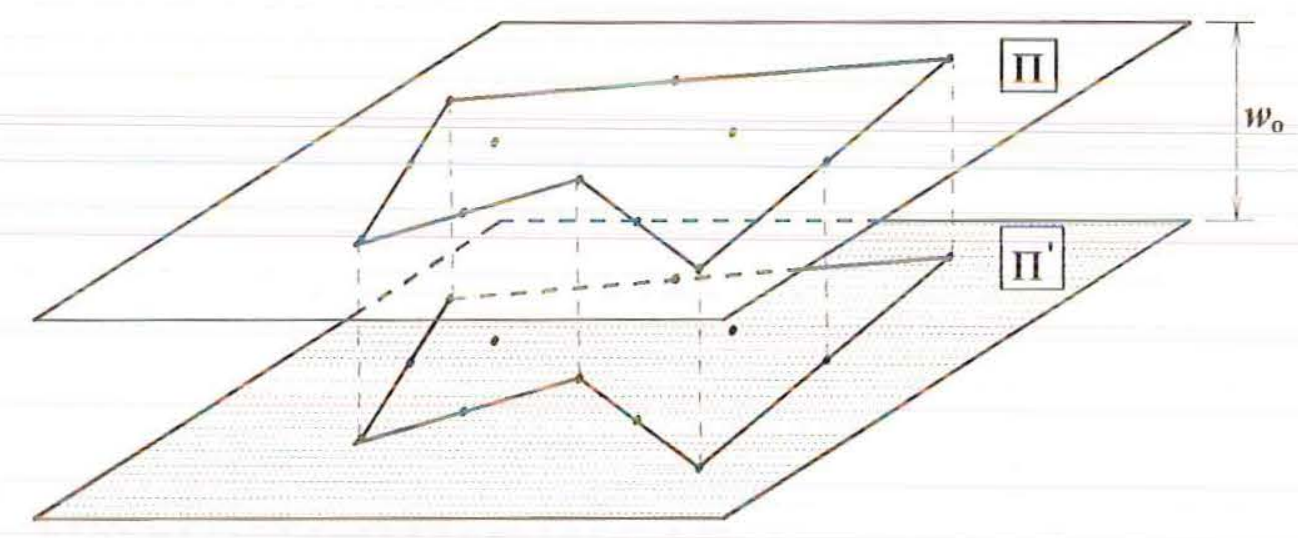

FIGURA 4.25 - Movimento de Corpo Rígido - Translação 


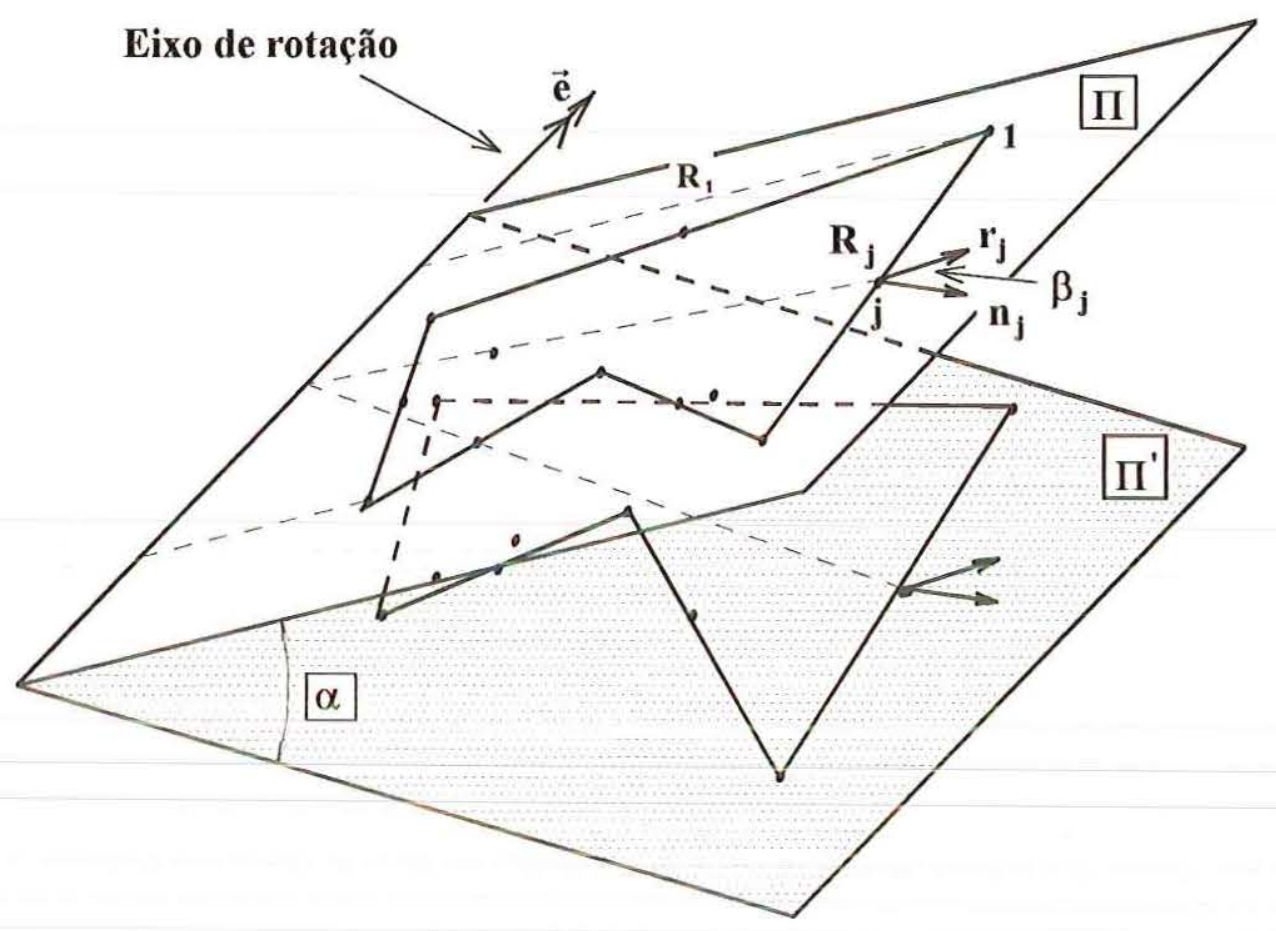

FIGURA 4.26 - Movimento de Corpo Rígido - Rotação.

Considerando-se um movimento vertical na placa, o deslocamento de corpo rígido $w_{0}$ nos nós é dado por:

$\underset{\sim}{\mathrm{U}^{\mathrm{T}}}=\left\{\begin{array}{llllllll}w_{\mathrm{o}} & 0 & w_{\mathrm{o}} & 0 & \ldots & \ldots & w_{\mathrm{o}} & 0\end{array}\right\}$

$\underset{\sim}{w_{\mathrm{c}}^{\mathrm{T}}}=\left\{\begin{array}{lllll}w_{\mathrm{o}} & w_{\mathrm{o}} & w_{\mathrm{o}} & \ldots & w_{\mathrm{o}}\end{array}\right\}$

$\underset{\sim}{\mathrm{U}_{\mathrm{i}}}=\left\{\begin{array}{lllll}w_{\mathrm{o}} & w_{\mathrm{o}} & w_{\mathrm{o}} & \ldots & w_{\mathrm{o}}\end{array}\right\}$ 
Substituindo-se estes valores em (4.94), resulta na propriedade, envolvendo os elementos das colunas ímpares, para qualquer linha $\mathbf{i}$ da matriz $\mathbf{H}_{\mathbf{b}}$,e envolvendo os elementos de todas a colunas das matrizes $\mathbf{H}_{\mathbf{c}}$ e $\mathbf{H}_{\mathbf{i}}$ :

$$
\sum_{j=1}^{N n} h_{1,2 j-1}+\sum_{j=1}^{N c} h_{c i, j}+\sum_{j=1}^{N p i} h_{i i, j}=0
$$

Considerando-se agora uma rotação de corpo rígido $\alpha$, figura 2.26 , no sentido indicado pelo eixo arbitrário $\overrightarrow{\mathbf{e}}$, o vetor deslocamento fica:

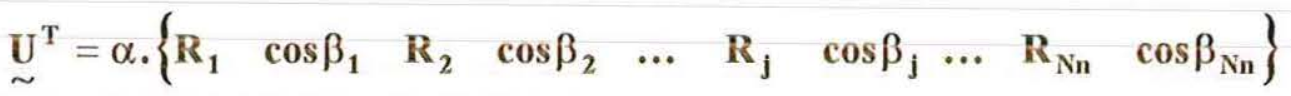

$$
\begin{aligned}
& \underset{\sim}{w_{\mathrm{c}}^{\mathrm{T}}}=\alpha \cdot\left\{\begin{array}{lllll}
\mathbf{R}_{\mathrm{c} 1} & \mathbf{R}_{\mathrm{c} 2} & \mathbf{R}_{\mathrm{c} 3} & \ldots & \mathbf{R}_{\mathrm{cNc}}
\end{array}\right\} \\
& \underset{\sim}{\mathbf{U}_{\mathrm{i}}}=\left\{\begin{array}{lllll}
\mathbf{R}_{\mathrm{i} 1} & \mathbf{R}_{\mathrm{i} 2} & \mathbf{R}_{\mathrm{i} 3} & \ldots & \mathbf{R}_{\mathrm{iNpi}}
\end{array}\right\}
\end{aligned}
$$

onde $\mathbf{R}_{\mathbf{j}}$ é a distância entre um nó genérico j e o eixo de rotação e $\cos \beta_{\mathrm{j}}=\mathbf{n}_{\mathrm{k}}^{\mathrm{j}} \cdot \mathbf{R}_{\mathrm{k}}$.

\subsection{Deslocamentos e Esforços para Pontos Internos}

Após a determinação dos deslocamentos e esforços no contorno da placa, é necessária a obtenção destes valores para pontos do interior da placa.

O deslocamento $w(q)$ em um ponto do interior q é dado pela equação (3.40) para o caso de placa com espessura variável e quando a rigidez for constante esta equação recai na equação (3.22). A partir da equação (3.40), pode-se chegar às representações integrais das curvaturas de pontos internos, segundo as direções $\mathbf{x}_{\mathbf{1}} \mathrm{e}$ $\mathbf{x}_{2}$ de um sistema cartesiano com origem no ponto q. Assim, tem-se que: 


$$
\begin{aligned}
& w_{q_{\mathrm{ij}}}(\mathbf{q})=-\int_{\Gamma}\left\{\frac{1}{\mathbf{D q}} \cdot\left[\mathbf{D}(\mathbf{s}) \cdot \frac{\partial^{2} \mathbf{V}_{\mathrm{n}}^{*}}{\partial \mathbf{x}_{\mathbf{i}} \cdot \partial \mathbf{x}_{\mathbf{j}}}(\mathbf{q}, \mathbf{p})+2 \cdot \frac{\partial \mathbf{D}}{\partial \mathbf{s}} \cdot \frac{\partial^{2} \mathbf{M}_{n \mathbf{s}}^{*}}{\partial \mathbf{x}_{\mathbf{i}} \cdot \partial \mathbf{x}_{\mathbf{j}}}(\mathbf{q}, \mathbf{p})+\frac{\partial \mathbf{D}}{\partial \mathbf{n}} \cdot \frac{\partial^{2} \mathbf{M}_{\mathbf{n}}^{*}}{\partial \mathbf{x}_{\mathbf{i}} \cdot \partial \mathbf{x}_{\mathbf{j}}}(\mathbf{q}, \mathbf{p})\right] \cdot w(\mathbf{p})\right. \\
& \left.-\frac{\mathbf{D}(\mathbf{s})}{\mathrm{Dq}} \cdot \frac{\partial^{2} \mathbf{M}_{\mathrm{n}}^{*}}{\partial \mathbf{x}_{\mathbf{i}} \cdot \partial \mathbf{x}_{\mathbf{j}}}(\mathbf{q}, \mathbf{p}) \cdot \frac{\partial w}{\partial \mathbf{n}}(\mathbf{p})\right\} \cdot \mathrm{d} \Gamma-\sum_{\mathrm{c}=1}^{N c} \frac{\partial^{2} \mathbf{R}_{\mathrm{cc}}^{*}}{\partial \mathbf{x}_{\mathrm{i}} \cdot \partial \mathbf{x}_{\mathbf{j}}}(\mathbf{q}, \mathbf{p}) \cdot w_{\mathrm{cc}}(\mathbf{p}) \cdot \frac{\mathbf{D}_{\mathrm{cc}}}{\mathbf{D q}} \\
& -\int_{\Omega} g(p) \cdot \frac{\partial^{2} \overline{\mathbf{K}}^{*}}{\partial \mathbf{x}_{\mathbf{i}} \cdot \partial \mathbf{x}_{\mathbf{j}}}(\mathbf{q}, \mathbf{p}) \cdot w(\mathbf{p}) \cdot d \Omega \\
& +\frac{D o}{D q} \cdot \int\left[\frac{\partial^{2} w^{*}}{\partial \mathbf{x}_{i} \cdot \partial \mathbf{x}_{j}}(q, p) \cdot V_{n}(p)-M_{n}(p) \cdot \frac{\partial^{2}}{\partial \mathbf{x}_{i} \cdot \partial x_{j}}\left(\frac{\partial w^{*}}{\partial n}(p)\right)\right] \cdot d \Gamma \\
& +\frac{D o}{D q} \cdot \sum_{c=1}^{N c} R_{c c}(p) \cdot \frac{\partial^{2} w_{c c}^{*}}{\partial x_{i} \cdot \partial x_{j}}(q, p)+\frac{D o}{D q_{\Omega_{g}}} \int_{g} g(p) \cdot \frac{\partial^{2} w}{\partial x_{i} \cdot \partial x_{j}}(q, p) \cdot d \Omega_{g}
\end{aligned}
$$

As equações do deslocamento e das curvaturas podem ser escritas através de uma única equação, denominando-se, genericamente, os deslocamentos e esforços e suas derivadas, respectivamente, por u e q. Logo, as equações (3.40) e (4.102) são expressas, semelhantemente a (4.59), por:

$$
\begin{aligned}
& \underset{\sim}{\mathrm{u}}(\mathrm{q})+\int_{\Gamma} \overline{\mathrm{p}}^{*}(\mathrm{q}, \mathrm{P}) \cdot \underset{\sim}{\mathrm{u}}(\mathrm{P}) \cdot \mathrm{d} \Gamma(\mathrm{P})+\sum_{\mathrm{i}=1}^{\mathrm{Nc}} \overline{\mathrm{p}}_{\mathrm{c}}^{\mathrm{*}}(\mathrm{q}, \mathrm{P}) \cdot w_{\mathrm{ci}}(\mathrm{P}) \\
& -\int_{\Omega_{g s}} \underset{\sim}{\aleph^{*}}(q, P) \cdot u_{g}(p) \cdot d \Omega_{g s}(p)=\int_{\sim}^{u^{*}}(q, P) \cdot p(P), d \Gamma(P)+\sum_{i=1}^{N c} u_{c i}^{*}(q, P) \cdot R_{c i}(P) \\
& +\int_{\Omega_{g}} g(p) \cdot u_{g}^{*}(q, P) \cdot d \Omega_{g}(p)
\end{aligned}
$$


onde:

$$
\underset{\sim}{\mathbf{u}(q)}=\left\{\begin{array}{c}
w(q) \\
\frac{\partial^{2} w(q)}{\partial \mathrm{x}_{1} \cdot \partial \mathrm{x}_{1}} \\
\frac{\partial^{2} w(q)}{\partial \mathrm{x}_{1} \cdot \partial \mathrm{x}_{2}} \\
\frac{\partial^{2} w(q)}{\partial \mathrm{x}_{2} \cdot \partial \mathrm{x}_{2}}
\end{array}\right\}
$$



$$
\bar{\Re}^{*}(q, P)=\left[\frac{1}{D 0} \cdot\left(\mathbf{D}(s) \cdot V_{n}^{*}(q, P)+2 \cdot \frac{\partial D(s)}{\partial s} \cdot M_{n s}^{*}(q, P)+\frac{\partial D(s)}{\partial n} \cdot M_{n}^{*}(q, P)\right)\right]
$$

$$
\frac{\partial^{2} \overline{\mathbb{R}}^{*}}{\partial \mathbf{x}_{i} \cdot \partial \mathbf{x}_{j}}=\left[\frac{1}{\mathbf{D o}} \cdot\left(\mathbf{D}(s) \cdot \frac{\partial^{2} \mathbf{V}_{n}^{n}(\mathbf{q}, \mathbf{P})}{\partial \mathbf{x}_{i} \cdot \partial \mathbf{x}_{j}}+2 \cdot \frac{\partial \mathbf{D}(s)}{\partial s} \cdot \frac{\partial^{2} \mathbf{M}_{n s}^{n}(\mathbf{q}, \mathbf{P})}{\partial \mathbf{x}_{i} \cdot \partial \mathbf{x}_{j}}+\frac{\partial \mathbf{D}(s)}{\partial \mathbf{n}} \cdot \frac{\partial^{2} \mathbf{M}_{n}^{n}(\mathbf{q}, \mathbf{P})}{\partial \mathbf{x}_{i} \cdot \partial \mathbf{x}_{j}}\right)\right]
$$




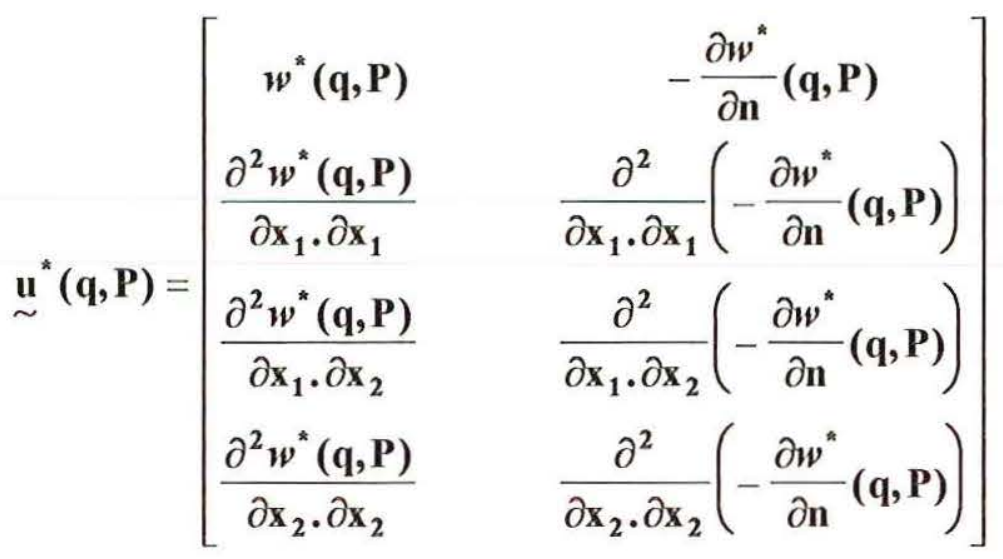

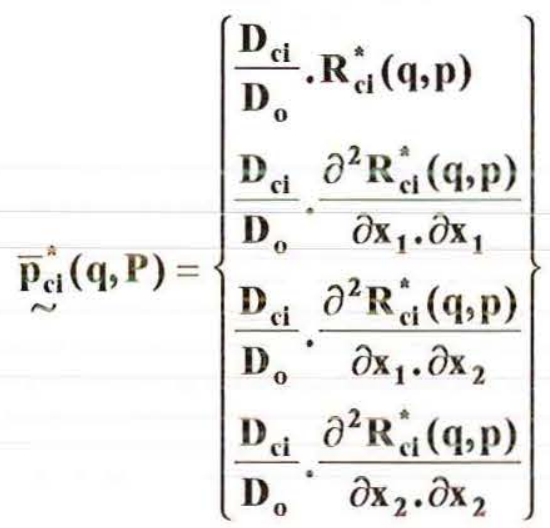

$$
\underset{\sim}{\sim}(q, p)=\left\{\begin{array}{l}
\overline{\mathbf{K}}^{*}(\mathbf{q}, \mathbf{p}) \\
\frac{\partial^{2} \overline{\mathbf{K}}^{*}(\mathbf{q}, \mathbf{p})}{\partial \mathrm{x}_{1} \cdot \partial \mathbf{x}_{1}} \\
\frac{\partial^{2} \overline{\mathbf{K}}^{*}(\mathbf{q}, \mathbf{p})}{\partial \mathrm{x}_{1} \cdot \partial \mathbf{x}_{2}} \\
\frac{\partial^{2} \overline{\mathbf{K}}^{*}(\mathbf{q}, \mathbf{p})}{\partial \mathbf{x}_{2} \cdot \partial \mathbf{x}_{2}}
\end{array}\right\}
$$

$$
\overline{\mathrm{K}}^{*}(\mathrm{q}, \mathrm{p})=2 \cdot \frac{\partial \mathrm{D}(\mathrm{s})}{\partial \mathrm{x}_{\ell}} \cdot \mathrm{q}_{\ell}^{*}(\mathrm{q}, \mathrm{p})+\frac{\partial \mathrm{D}(\mathrm{s})}{\partial \mathrm{x}_{\ell} \cdot \partial \mathrm{x}_{\mathrm{k}}} \cdot \mathrm{m}_{\ell \mathrm{k}}^{*}(\mathrm{q}, \mathrm{p})
$$

$$
\frac{\partial^{2} \overline{\mathbf{K}}^{*}}{\partial \mathbf{x}_{\mathbf{i}} \cdot \partial \mathbf{x}_{\mathbf{j}}}(\mathbf{q}, \mathbf{p})=\frac{\partial^{2}}{\partial \mathbf{x}_{\mathbf{i}} \cdot \partial \mathbf{x}_{\mathbf{j}}}\left(2 \cdot \frac{\partial \mathbf{D}(s)}{\partial \mathbf{x}_{\ell}} \cdot \mathbf{q}_{\ell}^{*}(\mathbf{q}, \mathbf{p})\right)+\frac{\partial^{2}}{\partial \mathbf{x}_{\mathbf{i}} \cdot \partial \mathbf{x}_{\mathbf{j}}}\left(\frac{\partial \mathbf{D}(\mathrm{s})}{\partial \mathbf{x}_{\ell} \cdot \partial \mathbf{x}_{\mathbf{k}}} \cdot \mathbf{m}_{\ell \mathbf{k}}^{*}(\mathbf{q}, \mathbf{p})\right)
$$




$$
\underset{\sim}{\mathbf{u}_{\mathrm{g}}^{*}(\mathbf{q}, \mathbf{p})}=\left\{\begin{array}{l}
w^{*}(\mathbf{q}, \mathbf{p}) \\
\frac{\partial^{2} w^{*}(\mathbf{q}, \mathbf{p})}{\partial \mathbf{x}_{1} \cdot \partial \mathbf{x}_{1}} \\
\frac{\partial^{2} w^{*}(\mathbf{q}, \mathbf{p})}{\partial \mathbf{x}_{1} \cdot \partial \mathbf{x}_{2}} \\
\frac{\partial^{2} w^{*}(\mathbf{q}, \mathbf{p})}{\partial \mathbf{x}_{2} \cdot \partial \mathrm{x}_{2}}
\end{array}\right\}
$$

$$
\underset{\sim}{\mathrm{u}_{\mathrm{ci}}^{\mathrm{*}}}(\mathrm{q}, \mathrm{P})=\left\{\begin{array}{c}
\frac{\mathrm{D}_{\mathrm{ci}}}{\mathrm{D}_{\mathrm{o}}} \cdot w_{\mathrm{ci}}^{*} \\
\frac{\mathrm{D}_{\mathrm{ci}}}{\mathrm{D}_{\mathrm{o}}} \cdot \frac{\partial^{2} w_{\mathrm{ci}}^{*}}{\partial \mathrm{x}_{1} \cdot \partial \mathrm{x}_{1}} \\
\frac{\mathrm{D}_{\mathrm{ci}}}{\mathrm{D}_{\mathrm{o}}} \cdot \frac{\partial^{2} w_{\mathrm{ci}}^{*}}{\partial \mathrm{x}_{1} \cdot \partial \mathrm{x}_{2}} \\
\frac{\mathrm{D}_{\mathrm{ci}}}{\mathrm{D}_{\mathrm{o}}} \cdot \frac{\partial^{2} w_{\mathrm{ci}}^{*}}{\partial \mathrm{x}_{2} \cdot \partial \mathrm{x}_{2}}
\end{array}\right\}
$$

Para problemas de placas de Kirchhoff com variação de espessura, há a necessidade de pontos internos como incógnitas, como já visto anteriormente, não sendo necessário calcular o deslocamento, neste ponto.

A parcela de domínio que contém o termo $\bar{\aleph}^{*}(q, p)$, pode ser eliminada da equação da curvatura, porque a derivada segunda de $\bar{K}^{*}(q, p)$ retorna valor nulo. Como será visto mais adiante nas derivadas das funções.

Após a discretização do contorno da placa, conforme descrito anteriormente, podem-se determinar os deslocamentos para outros pontos internos que não foram incógnita do problema, chagando-se para isso, a uma equação matricial dada por: 


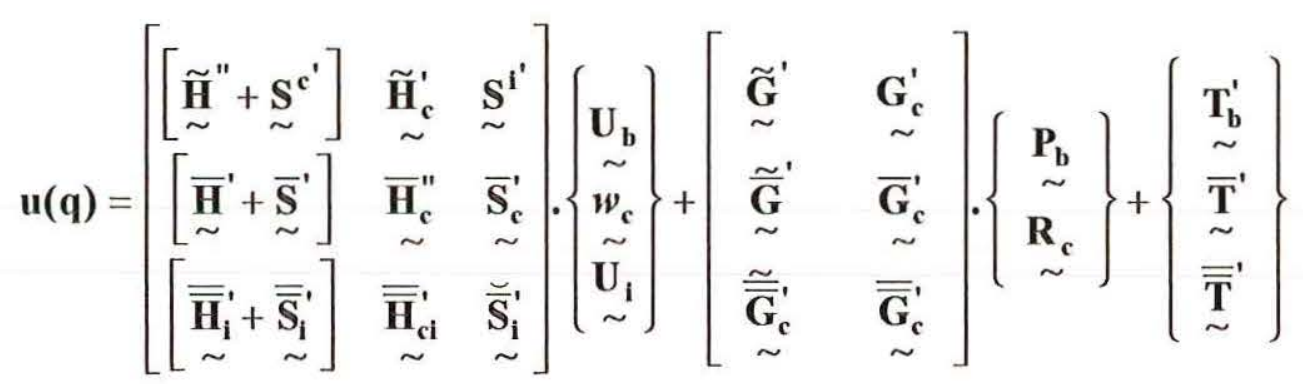

sendo $\mathbf{U}_{\mathbf{b}}$ e $\mathbf{P}_{\mathbf{b}}$ os vetores que contêm os deslocamentos e esforços dos nós do contorno e, portanto, já foram determinados anteriormente, assim como $w_{\mathrm{c}}$ e $\mathbf{R}_{\mathrm{c}}$, que são vetores dos deslocamentos e reações dos cantos da placa e também $\mathbf{U}_{\mathbf{i}}$ que são os nós internos que foram incógnitas do sistema.

Os momentos fletores e as forças cortantes para os pontos internos podem ser obtidos a partir das curvaturas, através das equações (2.22) e (2.24), respectivamente.

Substituindo-se os valores das curvaturas (4.102) em (2.22) e (2.24), obtêmse, explicitamente, os esforços para um ponto q no interior da placa, expressos por:

$$
\mathbf{m}_{i j}(\mathbf{q})=-\int_{\Gamma}\left(\overline{\mathbf{V}}_{n_{i j}}^{*}(\mathbf{q}, \mathbf{P})+2 \cdot \frac{\partial \mathbf{D}(s)}{\partial s} \cdot \mathbf{M}_{n_{i j}}^{*}(\mathbf{q}, \mathbf{P})+\frac{\partial \mathbf{D}(s)}{\partial n} \cdot \mathbf{M}_{n_{i j}}^{*}(\mathbf{q}, \mathbf{P})\right) \cdot w(\mathbf{P}) \cdot d \Gamma
$$

$$
\begin{aligned}
-\int_{\Gamma} \mathbf{D}(\mathrm{s}) \cdot \mathbf{M}_{\mathrm{n}_{\mathrm{ij}}}^{\mathrm{k}}(\mathbf{q}, \mathbf{P}) \cdot \frac{\partial w}{\partial \mathrm{n}}(\mathbf{P}) \cdot \mathrm{d} \Gamma-\sum_{\mathrm{i}=1}^{N_{\mathrm{c}}} \overline{\mathbf{R}}_{\mathrm{ci}}^{\mathrm{k}}(\mathbf{q}, \mathbf{P}) \cdot w_{\mathrm{ci}}(\mathbf{P}) \\
+\int_{\Gamma}\left(\mathbf{V}_{\mathrm{n}}(\mathbf{P}) \cdot w_{\mathrm{ij}}^{\mathrm{n}}(\mathbf{q}, \mathbf{P})-\mathbf{M}_{\mathrm{n}}(\mathbf{P}) \cdot \frac{\partial w_{\mathrm{ij}}^{\mathrm{k}}(\mathbf{q}, \mathbf{P})}{\partial \mathrm{n}}\right) \cdot \mathrm{d} \Gamma+\sum_{\mathrm{i}=1}^{\mathrm{N}_{\mathrm{c}}} \mathbf{R}_{\mathrm{ci}}(\mathbf{P}) \cdot w_{\mathrm{cij}}^{*}(\mathbf{q}, \mathbf{P}) \\
+\int_{\Omega_{\mathrm{g}}} \mathrm{g}(\mathrm{p}) \cdot w_{\mathrm{ij}}^{*}(\mathbf{q}, \mathrm{p}) \cdot \mathrm{d} \Omega_{\mathrm{g}}
\end{aligned}
$$




$$
\begin{aligned}
& \mathbf{q}_{\mathbf{j}}(\mathbf{q})=\int_{\Gamma}\left(\mathbf{D}(\mathbf{s}) \cdot \frac{\partial}{\partial \mathbf{x}_{\mathbf{i}}}\left(\frac{\partial^{2} \mathbf{V}_{\mathrm{n}}^{*}}{\partial \mathbf{x}_{\mathbf{k}} \cdot \partial \mathbf{x}_{\mathbf{k}}}(\mathbf{q}, \mathbf{p})\right)+2 \cdot \frac{\partial \mathbf{D}(\mathbf{s})}{\partial s} \cdot \frac{\partial}{\partial \mathbf{x}_{\mathbf{j}}}\left(\frac{\partial^{2} \mathbf{M}_{\mathrm{n}}^{*}}{\partial \mathbf{x}_{\mathbf{k}} \cdot \partial \mathbf{x}_{\mathbf{k}}}(\mathbf{q}, \mathbf{p})\right)\right. \\
& \left.+\frac{\partial \mathbf{D}(\mathbf{s})}{\partial \mathbf{n}} \cdot \frac{\partial}{\partial \mathbf{x}_{\mathbf{j}}}\left(\frac{\mathbf{M}_{\mathbf{n}}^{*}}{\partial \mathbf{x}_{\mathbf{k}} \cdot \partial \mathbf{x}_{\mathbf{k}}}(\mathbf{q}, \mathbf{p})\right)\right) \cdot w(\mathbf{p}) \cdot \mathbf{d} \Gamma(\mathbf{p})+\int_{\Gamma} \frac{\partial}{\partial \mathbf{x}_{\mathbf{j}}}\left(\frac{\partial^{2} \mathbf{M}_{\mathbf{n}}^{*}}{\partial \mathbf{x}_{\mathbf{k}} \cdot \partial \mathbf{x}_{\mathbf{k}}}(\mathbf{q}, \mathbf{p})\right) \cdot \frac{\partial w}{\partial \mathbf{n}}(\mathbf{p}) \cdot \mathrm{d} \Gamma(\mathbf{p}) \\
& +\sum_{\mathrm{i}=1}^{N c} \frac{\partial}{\partial \mathrm{x}_{\mathrm{j}}}\left(\frac{\partial^{2} \mathbf{R}_{\mathrm{cc}}^{*}}{\partial \mathrm{x}_{\mathrm{k}} \cdot \partial \mathrm{x}_{\mathrm{k}}}(\mathbf{q}, \mathbf{p})\right) \cdot w_{\mathrm{cc}}(\mathbf{p}) \cdot \frac{\mathbf{D}_{\mathrm{cc}}}{\mathbf{D}_{\mathrm{o}}} \\
& +\int_{\Gamma}\left[\mathbf{V}_{n}(p) \cdot \frac{\partial}{\partial \mathbf{x}_{\mathbf{i}}}\left(\frac{\partial^{2} w^{*}}{\partial \mathbf{x}_{\mathbf{k}} \cdot \partial \mathbf{x}_{\mathbf{k}}}(\mathbf{q}, \mathbf{p})\right)-\mathbf{M}_{n}(\mathbf{p}) \cdot \frac{\partial}{\partial \mathbf{x}_{j}}\left(\frac{\partial^{2}}{\partial \mathbf{x}_{\mathbf{k}} \cdot \partial \mathbf{x}_{\mathbf{k}}}\left(\frac{\partial w^{*}}{\partial \mathrm{n}}(\mathbf{q}, p)\right)\right)\right] \cdot d \Gamma(p) \\
& +\sum \mathbf{R}_{c c}(p) \cdot \frac{\partial}{\partial x_{j}}\left(\frac{\partial^{2} w_{c c}^{*}}{\partial x_{k} \cdot \partial x_{k}}(q, p)\right)+\int_{\Omega} g(p) \cdot \frac{\partial}{\partial x_{j}}\left(\frac{\partial^{2} w^{*}}{\partial x_{k} \cdot \partial x_{k}}(q, p)\right) \cdot d \Omega
\end{aligned}
$$

onde:

$$
\begin{aligned}
& \bar{V}_{n_{i j}}^{*}(q, p)=-D(s) \cdot\left(v \cdot \delta_{i j} \cdot \frac{\partial^{2} V_{n}^{*}}{\partial \mathbf{x}_{v} \cdot \partial \mathbf{x}_{v}}(q, p)+(1-v) \cdot \frac{\partial^{2} \mathbf{V}_{n}^{*}}{\partial \mathbf{x}_{i} \cdot \partial \mathbf{x}_{j}}(q, p)\right) \\
& \overline{\mathbf{M}}_{\mathrm{ns}_{\mathrm{ij}}}^{\mathrm{n}}(\mathbf{q}, \mathbf{p})=-\frac{\partial \mathbf{D}(s)}{\partial s} \cdot\left(v \cdot \delta_{\mathrm{ij}} \cdot \frac{\partial^{2} \mathbf{M}_{n s}^{\mathrm{*}}}{\partial \mathbf{x}_{\mathbf{v}} \cdot \partial \mathbf{x}_{\mathbf{v}}}(\mathbf{q}, \mathbf{p})+(1-v) \cdot \frac{\partial^{2} \mathbf{M}_{n s}^{*}}{\partial \mathbf{x}_{\mathbf{i}} \cdot \partial \mathbf{x}_{\mathbf{j}}}(q, p)\right) \\
& \overline{\mathbf{M}}_{\mathbf{n}_{\mathrm{ij}}}^{\mathrm{*}}(\mathbf{q}, \mathbf{p})=-\mathbf{D}(\mathrm{s}) \cdot\left(v \cdot \delta_{\mathrm{ij}} \cdot \frac{\partial^{2} \mathbf{M}_{\mathrm{n}}^{*}}{\partial \mathbf{x}_{\mathbf{v}} \cdot \partial \mathbf{x}_{\mathbf{v}}}(\mathbf{q}, \mathrm{p})+(1-v) \cdot \frac{\partial^{2} \mathbf{M}_{\mathrm{n}}^{*}}{\partial \mathbf{x}_{\mathrm{i}} \cdot \partial \mathbf{x}_{\mathrm{j}}}(\mathrm{q}, \mathrm{p})\right) \\
& \overline{\mathbf{R}}_{c_{i j}}^{*}(q, p)=-\mathbf{D}_{\mathrm{ci}} \cdot\left(v \cdot \delta_{\mathrm{ij}} \cdot \frac{\partial^{2} \mathbf{R}_{\mathrm{cc}}^{*}}{\partial \mathrm{x}_{\mathbf{v}} \cdot \partial \mathrm{x}_{\mathrm{v}}}(\mathrm{q}, \mathrm{p})+(1-v) \cdot \frac{\partial^{2} \mathbf{R}_{\mathrm{cc}}^{*}}{\partial \mathrm{x}_{\mathrm{i}} \cdot \partial \mathrm{x}_{\mathrm{j}}}(\mathbf{q}, \mathrm{p})\right)
\end{aligned}
$$




$$
\begin{aligned}
& w_{, j \mathrm{j}}^{*}(\mathbf{q}, \mathbf{p})=-\mathbf{D}_{\mathbf{o}} \cdot\left(v \cdot \delta_{\mathrm{ij}} \cdot \frac{\partial^{2} w^{*}}{\partial \mathbf{x}_{\mathbf{v}} \cdot \partial \mathbf{x}_{\mathbf{v}}}(\mathbf{q}, \mathbf{p})+(1-v) \cdot \frac{\partial^{2} w^{*}}{\partial \mathbf{x}_{\mathbf{i}} \cdot \partial \mathbf{x}_{\mathbf{j}}}(\mathbf{q}, \mathbf{p})\right)
\end{aligned}
$$

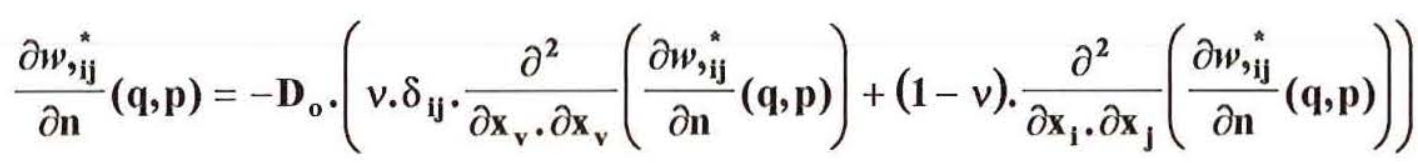

$$
\begin{aligned}
& w_{\mathrm{c}_{\mathrm{ij}}}^{*}(\mathbf{q}, \mathbf{p})=-\mathbf{D}_{\mathbf{0}} \cdot\left(v \cdot \delta_{\mathrm{ij}} \cdot \frac{\partial^{2} w_{\mathrm{c}_{i j}}^{*}}{\partial \mathbf{x}_{\mathrm{v}} \cdot \partial \mathbf{x}_{\mathrm{v}}}(\mathbf{q}, \mathbf{p})+(1-v) \cdot \frac{\partial^{2} w_{\mathrm{c}_{\mathrm{ij}}}^{*}}{\partial \mathbf{x}_{\mathrm{i}} \cdot \partial \mathbf{x}_{\mathrm{j}}}(\mathbf{q}, \mathbf{p})\right)
\end{aligned}
$$

As derivadas das soluções fundamentais que são necessárias, expressas nas equações (4.116) a (4.124), podem, ser determinadas em relação a um eixo particular $\mathbf{x}_{\mathbf{i}}$, definido no ponto $\mathbf{q}$; são dadas por:

$$
\frac{\partial w^{*}}{\partial \mathrm{x}_{\mathrm{i}}}(\mathrm{q}, \mathrm{p})=-\frac{\mathrm{r} \cdot \ln (\mathrm{r})}{4 \cdot \pi \cdot \mathrm{D} \mathbf{0}} \cdot \mathrm{r}_{\mathrm{i}}
$$

$$
\frac{\partial^{2} w^{*}}{\partial \mathrm{x}_{\mathrm{i}} \cdot \partial \mathrm{x}_{\mathrm{j}}}(\mathrm{q}, \mathrm{p})=\frac{1}{4 \cdot \pi \cdot \mathrm{Do}} \cdot\left(\mathrm{r}, \mathrm{i}, \mathrm{r}, \mathrm{j}, \delta_{\mathrm{ij}} \cdot \ln (\mathrm{r})\right)
$$

$$
\frac{\partial^{2} w^{*}}{\partial \mathrm{x}_{\mathrm{k}} \cdot \partial \mathrm{x}_{\mathrm{k}}}(\mathrm{q}, \mathrm{p})=\frac{1}{4 \cdot \pi \cdot \mathrm{D}_{0}} \cdot(1+2 \cdot \ln (\mathrm{r}))
$$

$$
\begin{aligned}
& \frac{\partial}{\partial \mathrm{x}_{\mathrm{i}}}\left(\frac{\partial^{2} w^{*}}{\partial \mathrm{x}_{\mathrm{k}} \cdot \partial \mathrm{x}_{\mathrm{k}}}(\mathbf{q}, \mathbf{p})\right)=\frac{-\mathrm{r}_{\mathbf{i}}}{2 \cdot \pi \cdot \text { Do. } \mathrm{r}} \\
& \frac{\partial}{\partial \mathrm{x}_{\mathrm{i}}}\left(\frac{\partial w^{*}}{\partial \mathrm{n}}\right)=\frac{-1}{4 \cdot \pi \cdot \mathrm{D}_{0}} \cdot\left[\mathrm{r}_{\mathrm{i}} \cdot\left(\mathrm{r}_{\mathrm{k}_{\mathrm{k}}} \cdot \mathrm{n}_{\mathrm{k}}\right)+\ln (\mathrm{r}) \cdot \mathrm{n}_{\mathrm{i}}\right]
\end{aligned}
$$

$$
\frac{\partial^{2}}{\partial \mathbf{x}_{i} \cdot \partial \mathrm{x}_{j}}\left(\frac{\partial w^{n}}{\partial \mathbf{n}}\right)=\frac{1}{4 \cdot \pi \cdot D_{0 \cdot r}} \cdot\left[\left(\delta_{i j}-2 \cdot r_{,}, r_{j}\right) \cdot\left(r_{, k} \cdot n_{k}\right)+r_{, i} \cdot n_{j}+r_{, j} \cdot n_{i}\right]
$$


4 EQUAÇÖES ALGÉBRICAS DO MEC PARA PLACA DE KIRCHHOFF

131

$$
\begin{aligned}
& \frac{\partial^{2}}{\partial \mathbf{x}_{\mathbf{k}} \cdot \partial \mathbf{x}_{\mathbf{k}}}\left(\frac{\partial w^{*}}{\partial \mathrm{n}}\right)=\frac{\mathbf{r}_{\mathbf{k}} \cdot \mathbf{n}_{\mathbf{k}}}{2 \cdot \pi \cdot \mathbf{D}_{0 . \mathbf{r}}} \\
& \frac{\partial}{\partial \mathbf{x}_{\mathbf{i}}}\left(\frac{\partial^{2}}{\partial \mathbf{x}_{\mathbf{k}} \cdot \partial \mathbf{x}_{\mathbf{k}}}\left(\frac{\partial w^{*}}{\partial \mathbf{n}}\right)\right)=\frac{1}{2 \cdot \pi \cdot \text { Do. }^{2}} \cdot\left[2 \cdot \mathbf{r}_{\mathrm{i}} \cdot\left(\mathbf{r},,_{k} \cdot \mathbf{n}_{k}\right)-n_{i}\right]
\end{aligned}
$$

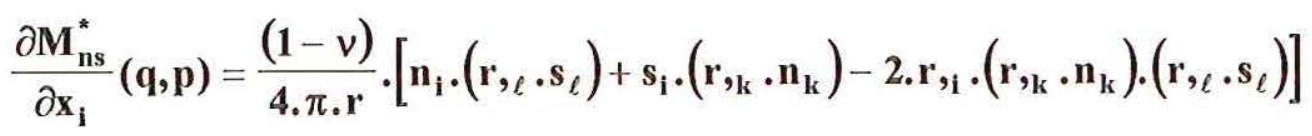

$$
\begin{aligned}
& \frac{\partial^{2} \mathbf{M}_{n s}^{*}}{\partial x_{1} \cdot \partial x_{j}}(q, p)=\frac{-(1-v)}{4 \cdot \pi \cdot r^{2}} \cdot\left\{\left[2 \cdot\left(\delta_{i j}+4 \cdot r,,_{i} \cdot r, j\right) \cdot\left(r, \ell_{\ell} \cdot s_{\ell}\right)-2 \cdot s_{i} \cdot r,,_{j}-2 \cdot r,,_{i} \cdot s_{j}\right] \cdot\left(r_{,_{k}} \cdot n_{k}\right)\right. \\
& \left.-2 \cdot\left(r_{j} \cdot n_{i}+r_{i} \cdot n_{j}\right) \cdot\left(r, r_{\ell} \cdot s_{\ell}\right) \cdot+n_{i} \cdot s_{j}+s_{i} \cdot n_{j}\right\} \\
& \frac{\partial^{2} \mathbf{M}_{n s}^{*}}{\partial \mathbf{x}_{\mathrm{k}} \cdot \partial \mathbf{x}_{\mathrm{k}}}(\mathbf{q}, \mathbf{p})=\frac{(1-v)}{\pi \cdot \mathbf{r}^{2}} \cdot\left\{\left(\mathrm{s}_{\ell} \cdot \mathbf{r}, \ell\right) \cdot\left(\mathbf{r}_{9_{\mathrm{k}}} \cdot \mathbf{n}_{\mathrm{k}}\right)\right\}
\end{aligned}
$$

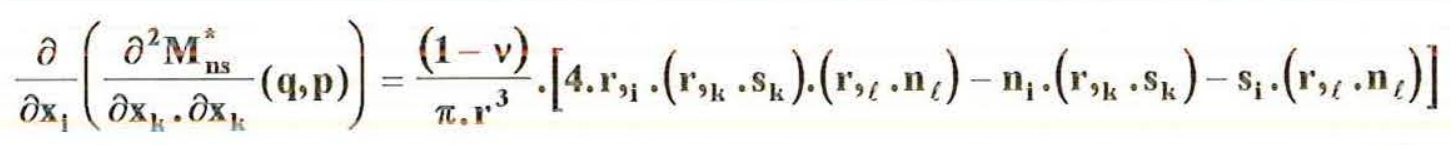

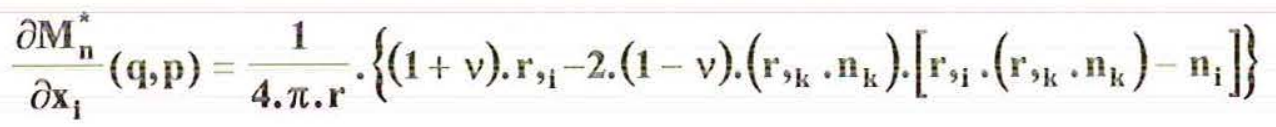

$$
\begin{aligned}
& \frac{\partial^{2} \mathbf{M}_{n}^{*}}{\partial \mathbf{x}_{i} \cdot \partial \mathbf{x}_{j}}(q, p)=-\frac{1}{4 \cdot \pi \cdot r^{2}} \cdot\left\{(1+v) \cdot\left(\delta_{i j}-2 \cdot r_{s_{i}} \cdot r_{, j}\right)\right. \\
& +2 \cdot(i-v) \cdot\left[n_{i} \cdot n_{j}-2 \cdot r,,_{j} \cdot n_{i} \cdot\left(r_{,_{k}} \cdot n_{k}\right)-\left(\delta_{i j}-2 \cdot r_{, j} \cdot r_{, k}\right) \cdot\left(r_{r_{k}} \cdot n_{k}\right)^{2}\right. \\
& \left.\left.-2 \cdot r_{q_{i}} \cdot\left(r_{9_{k}} \cdot n_{k}\right) \cdot\left[n_{j}-r_{9_{j}} \cdot\left(r_{9_{k}} \cdot n_{k}\right)\right]\right]\right\}
\end{aligned}
$$

(4.138) 
4 EQUAÇÖES ALGÉBRICAS DO MEC PARA PLACA DE KIRCHHOFF

132

$$
\begin{aligned}
& \frac{\partial^{2} M_{n}^{*}}{\partial x_{k} \cdot \partial x_{k}}(q, p)=-\frac{1}{4 \cdot \pi \cdot r^{2}} \cdot\left\{2 \cdot(1-v) \cdot\left[1-2 \cdot\left(r,,_{k} \cdot n_{k}\right)^{2}\right]\right\} \\
& \frac{\partial}{\partial x_{i}}\left(\frac{\partial^{2} M_{n}^{*}}{\partial x_{k} \cdot \partial x_{k}}(q, p)\right)=\frac{-(1-v)}{\pi \cdot r^{3}} \cdot\left\{r_{i_{i}}+2 \cdot n_{i} \cdot\left(r_{,_{k}} \cdot n_{k}\right)-4 \cdot r_{i_{i}} \cdot\left(r_{,_{k}} \cdot n_{k}\right)^{2}\right\}
\end{aligned}
$$

$$
\begin{aligned}
\frac{\partial V_{n}^{*}}{\partial x_{i}}(q, p)= & \frac{1}{4 \cdot \pi \cdot r^{2}} \cdot\left\{2 \cdot(1-v) \cdot\left(r,,_{\ell} \cdot s_{\ell}\right)^{2} \cdot\left[4 \cdot r,,_{i} \cdot\left(r,,_{k} \cdot n_{k}\right)-n_{i}\right]\right. \\
& \left.-4 \cdot(1-v) \cdot\left(r, r_{\ell}\right) \cdot\left(r_{,_{k}} \cdot n_{k}\right) \cdot s_{1}+(3-v) \cdot\left[n_{i}-2 \cdot r,,_{i} \cdot\left(r,,_{k} \cdot n_{k}\right)\right]\right\}
\end{aligned}
$$

$$
\begin{aligned}
& \frac{\partial^{2} V_{n}^{*}}{\partial x_{i} \cdot \partial x_{j}}(q, p)=\frac{1}{4 \cdot \pi \cdot r^{3}} \cdot\left\{2 \cdot ( 1 - v ) \cdot ( r _ { r _ { \ell } } \cdot s _ { \ell } ) ^ { 2 } \cdot \left[24, r_{,} \cdot r_{, j} \cdot\left(r,,_{k} \cdot n_{k}\right)\right.\right. \\
& \left.-4 \cdot\left[r,{ }_{i} \cdot n_{j}+r,{ }_{j} \cdot n_{i}+\delta_{i j} \cdot\left(r,,_{k} \cdot n_{k}\right)\right]\right]+2 \cdot(1-v) \cdot\left(r, r_{\ell} \cdot s_{\ell}\right) \cdot\left[2 \cdot\left(n_{i} \cdot s_{j}+n_{j} \cdot s_{i}\right)\right. \\
& \left.-8 \cdot\left(r_{9_{k}} \cdot n_{k}\right) \cdot\left(r_{9_{i}} \cdot s_{j}+r_{, j} \cdot s_{i}\right)\right]+4 \cdot(1-v) \cdot\left(r_{9_{k}} \cdot n_{k}\right) \cdot\left(s_{i} \cdot s_{j}\right)
\end{aligned}
$$

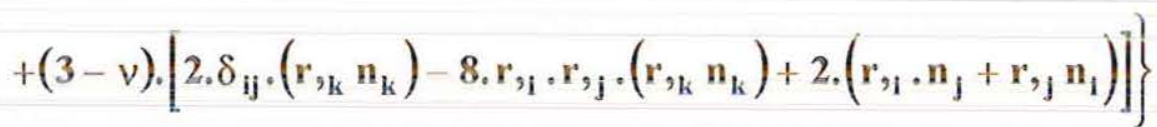

$$
\begin{aligned}
& \frac{\partial^{2} V_{n}^{*}}{\partial x_{k} \cdot \partial x_{k}}(q, p)=-\frac{(1-v)}{4 \cdot \pi \cdot r^{3}} \cdot\left(r_{, k} \cdot n_{k}\right) \cdot\left[4 \cdot\left(r, s_{\ell}\right)^{2}-1\right] \\
& \frac{\partial}{\partial x_{i}}\left(\frac{\partial^{2} V_{n}^{*}}{\partial x_{k} \cdot \partial x_{k}}(q, p)\right)=-\frac{(1-v)}{\pi \cdot r^{4}} \cdot\left\{\left(r,,_{\ell} \cdot s_{\ell}\right)^{2} \cdot\left[24 \cdot r,,_{i} \cdot\left(r,,_{k} \cdot n_{k}\right)-4 \cdot n_{i}\right]\right. \\
& \left.-\left(r_{,_{k}} \cdot n_{k}\right) \cdot\left[8 \cdot s_{i} \cdot\left(r_{\ell} \cdot s_{\ell}\right)+4 \cdot r_{,_{i}}\right]+n_{i}\right\}
\end{aligned}
$$


Com a discretização do contorno e a aproximação das variáveis e, ainda com a aplicação das equações (4.116) e (4.117) para Ni pontos internos, obtêm-se os esforços nestes pontos, através da equação matricial:

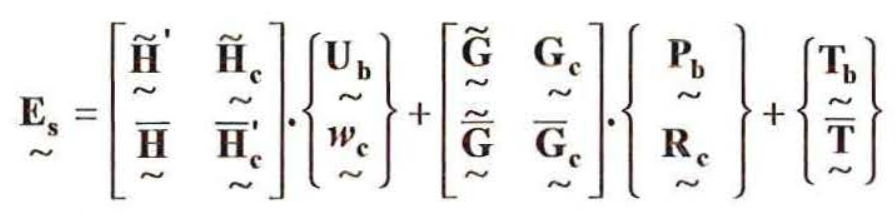

\section{9 integração sobre os Elementos}

A integração sobre os elementos será feita numericamente, adotando-se a quadratura de Gauss-Legendre, quando o ponto de colocação não pertence ao elemento de integração.

Quando o ponto de colocação está muito próximo ao elemento de integração, o resultado da quadratura não é obtido com a devida precisão mesmo que aumente bastante o número de pontos de Gauss. Estas integrais são denominadas de quase singulares. Uma maneira de evitar esta quase singularidade é utilizando subelementos.

Quando o ponto de colocação estiver sobre o elemento de integração, a integração será feita analiticamente.

\subsubsection{Integração Numérica}

A integração numérica será utilizada para os casos em que o ponto Q não pertence ao elemento a ser integrado, ou para um ponto A fora do domínio da placa. A integração é dada pela seguinte expressão: 


$$
\int_{a}^{b} f(\Gamma) \cdot d \Gamma=\sum_{i=1}^{N g} W_{i} \cdot f\left(\Gamma_{i}\right)
$$

onde:

- $\mathbf{f}(\Gamma)$ é a função a ser integrada;

- $\mathbf{N}_{\mathrm{g}}$ é o número de pontos de integração;

- $\mathbf{W}_{\mathrm{i}}$ é o fator ponderador, também definido em função de $\mathbf{N}_{\mathrm{g}}$.

O número $\mathbf{N}_{\mathrm{g}}$ de pontos de integração deve ser escolhido em função de alguns parâmetros, tais como a distância entre o ponto $\mathbf{Q}$ de carregamento e o elemento a ser integrado, o comprimento do elemento e a função a ser integrado, ver GIL RODRIGUEZ (1986).

Para intervalos de integração $[\mathrm{a}, \mathrm{b}]$ diferentes com o mesmo número de pontos de Gauss, ter-se-á valores das abscissas $\Gamma_{\mathbf{i}}$ e ponderadores $\mathbf{W}_{\mathbf{i}}$ diferentes. Por conveniência estas integrais devem ser expressas em termos das coordenadas adimensionais $\xi[-1 ; 1]$.

Da relação de coordenadas expressa em (4.7), levando-se em conta a figura 4.3, pode-se escrever:

$$
\Gamma_{\mathbf{j}}=\xi \cdot \frac{\ell}{2}
$$

sendo $\ell$ o comprimento do elemento.

Para a mudança da variável, é necessário o determinante do Jacobiano da transformação, que tem o valor:

$$
|\mathbf{J}|=\frac{d \Gamma_{j}}{d \xi}=\frac{\ell}{2}
$$

Assim, a integral (4.146) fica: 


$$
\frac{\ell}{2} \cdot \int_{-1}^{1} f(\xi) \cdot d \xi=\sum_{i=1}^{N g} W_{i} \cdot f\left(\xi_{i}\right)
$$

As integrais de contorno (4.72) e (4.73), necessárias para a montagem do sistema de equações (4.86) para obtenção dos valores de contorno são explicitamente dada por:

como:

$\mathbf{h}_{1}^{\mathrm{n}}(\mathbf{Q})=\frac{\ell}{2 \cdot \mathrm{D}_{\mathrm{o}}} \int_{-1}^{1}\left[\left(\mathrm{D}(\mathrm{s}) \cdot \mathbf{V}_{\mathrm{n}}^{*}(\mathbf{Q}, \mathbf{P})+2 \cdot \frac{\partial \mathrm{D}}{\partial \mathrm{s}} \cdot \mathbf{M}_{\mathrm{ns}}^{*}(\mathbf{Q}, \mathbf{P})+\frac{\partial \mathrm{D}}{\partial \mathbf{n}} \cdot \mathbf{M}_{\mathrm{n}}^{*}(\mathbf{Q}, \mathbf{P})\right) \cdot \phi_{\mathrm{n}}(\mathbf{P}) \cdot \mathrm{d} \xi(\mathbf{P})\right.$

$\mathbf{h}_{2}^{\mathrm{n}}(\mathrm{Q})=-\frac{\ell}{2} \int_{-1}^{1}\left(\frac{\mathrm{D}(\mathrm{s})}{\mathrm{D} 0} \cdot \mathrm{I}_{\mathrm{n}}^{*}(\mathrm{Q}, \mathrm{P})\right) \cdot \phi_{\mathrm{n}}(\mathrm{P}) \cdot \mathrm{d} \xi(\mathrm{P})$

$\mathrm{g}_{1}^{\mathrm{n}}(\mathbf{Q})=\frac{\ell}{2} \int_{-1}^{1} w^{*}(\mathbf{Q}, \mathbf{P}) \cdot \phi_{\mathrm{n}}(\mathrm{P}) \cdot \mathrm{d} \xi(\mathrm{P})$

$\mathrm{g}_{2}^{\mathrm{n}}(\mathbf{Q})=-\frac{\ell}{2} \int_{-1}^{1} \frac{\partial w^{*}}{\partial \mathrm{n}}(\mathbf{Q}, \mathbf{P}) \cdot \phi_{\mathrm{n}}(\mathbf{P}) \cdot \mathrm{d} \xi(\mathbf{P})$

onde:

- n representa o nó local do elemento ou onde se mede a resposta do carregamento unitário aplicado;

- Q é o ponto singular ou de carregamento, localizado no contorno. Será o ponto A, se estiver fora do domínio da placa.

\subsubsection{Integração Quase Singular}

A eficiência da quadratura empregada depende de outros fatores além do número de pontos de integração. A distância do ponto de colocação ao elemento de integração é um desses fatores. 
Uma maneira de evitar esta quase singularidade é a utilização de sub-elemento que consiste basicamente em dividir o elemento de integração em sub-elemento menores (de comprimento padronizado ou progressivo). Os sub-elementos padronizados, de tamanho iguais, não são tão eficientes quanto os sub-elementos progressivos, de tamanho variável, cujo tamanho é em função da distância do ponto de colocação, CHAVES (1997).

Considere-se a seguinte relação válida:

$$
\int_{\Gamma_{j}} f\left(\Gamma_{j}\right) \cdot \phi_{n} \cdot d \Gamma_{j}=\sum_{i=1}^{N s u b e l} \int_{\Gamma_{j_{i}}} f\left(\Gamma_{j_{i}}\right) \cdot \phi_{n} \cdot d \Gamma_{j_{i}}
$$

transformando-se para as coordenadas adimensionais $\eta$, isto é, para $\Gamma=\mathbf{a}_{\mathbf{i}} \rightarrow \eta=-\mathbf{1}$ e $\Gamma=\mathbf{b}_{\mathbf{i}} \rightarrow \eta=1$, figura 4.28, logo:

$$
\int_{\Gamma_{j}} f\left(\Gamma_{j}\right) \cdot \phi_{n} \cdot d \Gamma_{j}=\sum_{i=1}^{\text {Nsubel }} \frac{l_{i}}{2} \cdot \int_{-1}^{1} f(\eta) \cdot \phi_{n} \cdot d \eta
$$

Pode-se observar que $\phi_{\mathbf{n}}(\xi)$ está relacionado com a coordenada adimensional $\xi$, pois $\phi_{n}(\xi)$ são funçôes de forma, isto é, relaciona o valor de uma dada variável em função dos valores nodais do elemento, figura 4.27. 


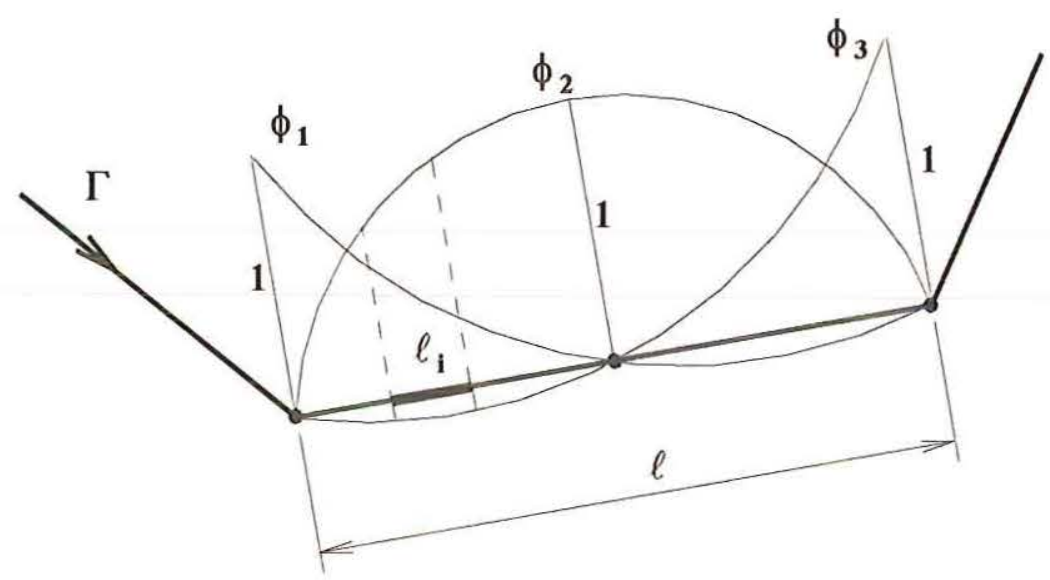

FIGURA 4.27 - Funções de Forma Global $\phi_{\mathbf{n}}$.

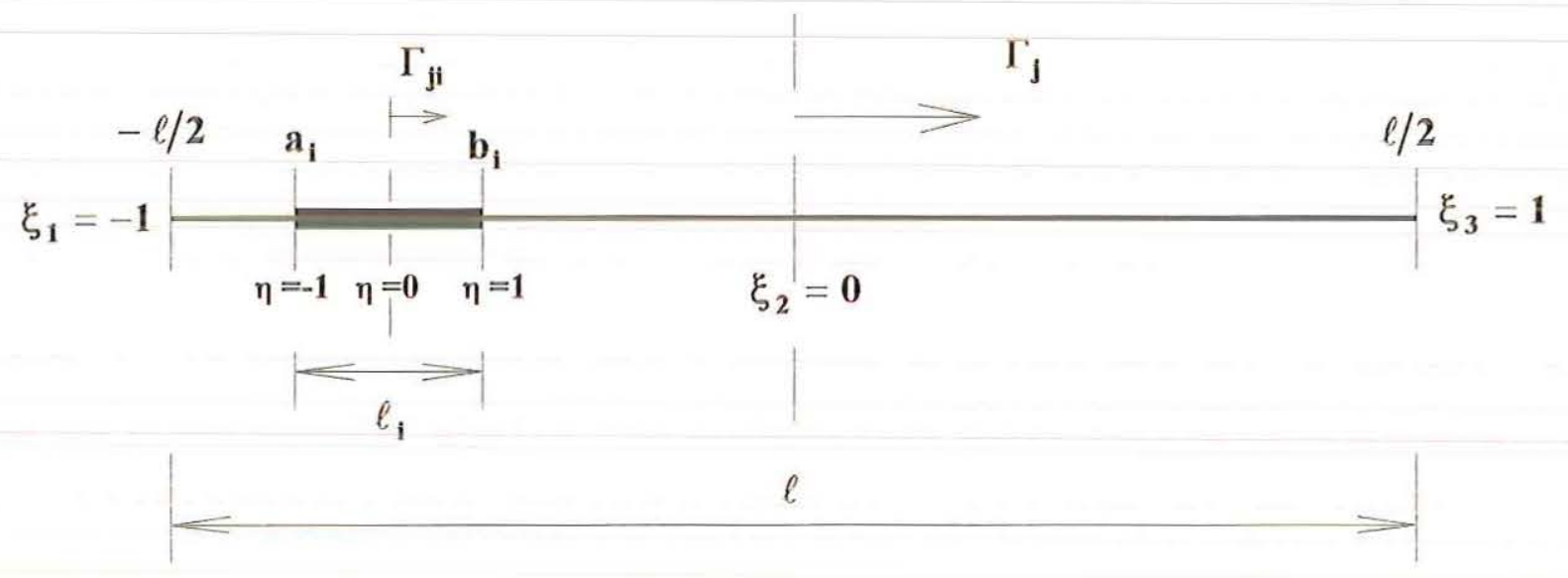

FIGURA 4.28 - Coordenadas Adimensionais $\eta$ e $\xi$.

Pelo fato do intervalo de integração estar em função da coordenada adimensional $\eta[-1 ; 1]$ e sendo a função de forma expressa em coordenada adimensional $\xi$, tem-se a necessidade de fazer a correlação entre as duas coordenadas adimensionais.

A subrotina para obtenção do subelemento utilizada neste trabalho foi gentilmente cedida por FERNANDES ${ }^{1}$. 


\subsubsection{Integração Singular}

Quando o ponto de colocação pertence ao elemento de integração, o integrando no cálculo das matrizes de influência apresentará uma singularidade devido à solução fundamental.

Podem estas integrais ser resolvidas numericamente, fazendo-se para isto mudança do polinomial do integrando eliminando assim a singularidade do integrando, podendo-se obter as abscissas e ponderadores.

Pode ainda, estas integrais, serem resolvidas analiticamente, sendo esta utilizada neste trabalho para eliminar a singularidade.

Devido a $\mathbf{r}_{\mathbf{i}} \cdot \mathbf{n}_{\mathbf{i}}=\mathbf{0}$ para o elemento singular as parcelas $\frac{\partial w^{*}}{\partial \mathbf{n}}, \mathbf{M}_{\mathbf{n}}^{*}$ e $\mathbf{V}_{\mathbf{n}}^{*}$ dadas pelas equações (2.96), (2.98) e (2.99), respectivamente, serão iguais a zero. Logo a integral dada em (4.150) a (4.153) resumem a, respectivamente:

$$
\begin{aligned}
& \mathbf{h}_{1}^{\mathrm{n}}=\frac{\ell}{2 . \mathrm{Do}} \cdot \frac{\partial \mathbf{D}}{\partial \mathbf{n}} \cdot \int_{-1}^{1} \mathbf{M}_{\mathbf{n}}^{\mathrm{n}}(\mathbf{Q}, \mathbf{P}) \cdot \phi_{\mathrm{n}}(\mathbf{P}) \cdot \mathrm{d} \xi(\mathbf{P}) \\
& h_{2}^{n}(Q)=-\frac{\ell}{2} \int_{-1}^{1}\left(\frac{D(s)}{D 0} \cdot M_{n}^{*}(Q, P)\right) \cdot \hat{\varphi}_{n}(P) \cdot d \xi(P) \\
& \mathrm{g}_{1}^{\mathrm{n}}(\mathbf{Q})=\frac{\ell}{2} \int_{-1}^{1} w^{*}(\mathbf{Q}, \mathbf{P}) \cdot \phi_{\mathrm{n}}(\mathbf{P}) \cdot \mathrm{d} \xi(\mathbf{P}) \\
& \mathrm{g}_{2}^{\mathrm{n}}(\mathrm{Q})=\mathbf{0}
\end{aligned}
$$

As expressões (4.156) a (4.157) para placas com espessura constante são dadas por:

$$
\mathbf{h}_{\mathbf{1}}^{\mathbf{n}}=\mathbf{0}
$$




$$
\begin{aligned}
& \mathbf{h}_{2}^{\mathrm{n}}(\mathbf{Q})=-\frac{\ell}{2} \int_{-1}^{1} M_{n}^{*}(Q, P) \cdot \phi_{n}(P) \cdot d \xi(P) \\
& \mathrm{g}_{1}^{\mathrm{n}}(\mathbf{Q})=\frac{\ell}{2} \int_{-1}^{1} w^{*}(Q, P) \cdot \phi_{n}(P) \cdot d \xi(P) \\
& \mathbf{g}_{2}^{\mathrm{n}}(\mathbf{Q})=0
\end{aligned}
$$

CHUEIRI (1994) deduziu as integrais analíticas (4.158) a (4.159) para elementos singulares, considerando-se o ponto singular numa posição genérica dada pela coordenada adimensional $\xi_{\mathrm{s}}$. Considerando-se também o elemento linear descontínuo, figura 4.29, obtendo-se como resultado:

$$
\mathrm{h}_{1}^{\mathrm{n}}(\mathrm{Q})=\mathbf{0}
$$

$$
\begin{aligned}
\mathbf{h}_{2}^{\mathrm{n}}(\mathbf{Q})= & \frac{\ell \mathbf{k}}{4 \cdot \pi} \cdot\left\{\mathbf{C}_{1}^{\mathrm{n}} \cdot(1+v) \cdot\left[\phi_{2 \mathrm{~s}} \cdot \ln \left(\ell \mathbf{k} \cdot \phi_{2 \mathrm{~s}}\right)+\phi_{1 \mathrm{~s}} \cdot \ln \left(\ell \mathbf{k} \cdot \phi_{1 \mathrm{~s}}\right)-1\right]\right. \\
& +\mathbf{C}_{2}^{\mathrm{n}} \cdot(1+v) \cdot\left[\left(\phi_{2 \mathrm{~s}}\right)^{2} \cdot \ln \left(\ell \mathbf{k} \cdot \phi_{2 \mathrm{~s}}\right)-\left(\phi_{1 \mathrm{~s}}\right)^{2} \cdot \ln \left(\ell \mathbf{k} \cdot \phi_{1 \mathrm{~s}}\right)-\frac{\xi_{\mathrm{s}}}{2}\right]
\end{aligned}
$$

$$
\begin{gathered}
+\mathbf{C}_{3}^{\mathrm{n}} \cdot(1+v) \cdot\left[\frac{4}{3} \cdot\left(\phi_{2 \mathrm{~s}}\right)^{3} \cdot \ln \left(\ell \mathbf{k} \cdot \phi_{2 \mathrm{~s}}\right)+\frac{4}{3} \cdot\left(\phi_{1 \mathrm{~s}}\right)^{3} \cdot \ln \left(\ell k \cdot \phi_{1 \mathrm{~s}}\right)-\frac{1}{9}\left(1+3 \cdot \xi_{\mathrm{s}}^{2}\right)\right] \\
\left.+v \cdot\left[\mathrm{C}_{1}^{\mathrm{n}}+\mathbf{C}_{2}^{\mathrm{n}} \cdot \xi_{\mathrm{s}}+\frac{\mathrm{C}_{3}^{\mathrm{n}}}{3} \cdot\left(1+3 \cdot \xi_{\mathrm{s}}^{2}\right)\right]\right\}
\end{gathered}
$$

$$
\mathrm{g}_{1}^{\mathrm{n}}(\mathrm{Q})=\frac{\ell \mathbf{k}^{3}}{8 \cdot \pi \cdot \mathrm{Do}_{0}} \cdot\left\{\frac{\mathrm{C}_{1}^{\mathrm{n}}}{3} \cdot\left[\left(\phi_{2 \mathrm{~s}}\right)^{2} \cdot\left(\ln \left(\ell \mathrm{k} \cdot \phi_{2 \mathrm{~s}}\right)-\frac{5}{6}\right)+\left(\phi_{1 \mathrm{~s}}\right)^{3} \cdot\left(\ln \left(\ell \mathrm{k} \cdot \phi_{1 \mathrm{~s}}\right)-\frac{5}{6}\right)\right]\right.
$$




$$
\begin{aligned}
& +\frac{\mathbf{C}_{2}^{\mathrm{n}}}{2} \cdot\left[\left(\phi_{2 \mathrm{~s}}\right)^{4} \cdot\left(\ln \left(\ell \mathbf{k} \cdot \phi_{2 \mathrm{~s}}\right)-\frac{3}{4}\right)-\left(\phi_{1 \mathrm{~s}}\right)^{4} \cdot\left(\ln \left(\ell \mathbf{k} \cdot \phi_{1 \mathrm{~s}}\right)-\frac{3}{4}\right)\right] \\
& \left.+\frac{4 \cdot \mathbf{C}_{3}^{\mathrm{n}}}{5} \cdot\left[\left(\phi_{2 \mathrm{~s}}\right)^{5} \cdot\left(\ln \left(\ell \mathrm{k} \cdot \phi_{2 \mathrm{~s}}\right)-\frac{7}{10}\right)+\left(\phi_{1 \mathrm{~s}}\right)^{5} \cdot\left(\ln \left(\ell \mathbf{k} \cdot \phi_{1 \mathrm{~s}}\right)-\frac{7}{10}\right)\right]\right\} \\
& \mathbf{g}_{2}^{\mathrm{n}}(\mathbf{Q})=0
\end{aligned}
$$

Nestas expressões, as constantes $\mathbf{C}_{\mathbf{1}}^{\mathbf{n}}, \mathbf{C}_{\mathbf{2}}^{\mathbf{n}}, \mathbf{C}_{\mathbf{3}}^{\mathbf{n}}$ são relativas aos nós locais e portanto, dependem das funções aproximadoras $\phi_{1}, \phi_{2}, \phi_{3}$. Estes valores podem ser escritos, genericamente, na forma:

$$
\begin{gathered}
\mathbf{C}_{1}^{\mathrm{n}}=\frac{-\xi_{\mathrm{s}}^{2}+\left(\xi_{\mathrm{i}}+\xi_{\mathrm{j}}\right) \cdot \xi_{\mathrm{s}}-\xi_{\mathrm{i}} \cdot \xi_{\mathrm{j}}}{\left(\xi_{\mathrm{i}}-\xi_{\mathrm{n}}\right) \cdot\left(\xi_{\mathrm{n}}-\xi_{\mathrm{j}}\right)} \\
\mathbf{C}_{2}^{n}=\frac{2 \cdot \xi_{\mathrm{s}}-\left(\xi_{\mathrm{i}}+\xi_{\mathrm{j}}\right)}{\left(\xi_{\mathrm{i}}-\xi_{\mathrm{n}}\right) \cdot\left(\xi_{\mathrm{n}}-\xi_{\mathrm{j}}\right)} \\
\mathbf{C}_{2}^{n}=\frac{-1}{\left(\xi_{i}-\xi_{n}\right) \cdot\left(\xi_{n}-\xi_{j}\right)}
\end{gathered}
$$

$\operatorname{Com} n, \mathbf{i}, \mathbf{j}=1,2,3$ e $n \neq i \neq j$ e,

$$
\begin{aligned}
\phi_{1 \mathrm{~s}} & =\frac{(1-\xi)}{2} \\
\phi_{2 \mathrm{~s}} & =\frac{(1+\xi)}{2}
\end{aligned}
$$

Neste trabalho adotou-se aproximação contínua para as variáveis, com isso as expressões (4.164) podem-se ser rescritas explicitamente como: 


$$
C_{1}^{1}=\frac{-\left(1-\xi_{s}\right) \cdot \xi_{s}}{2} \quad C_{1}^{2}=1-\xi_{s}^{2} \quad C_{1}^{3}=\frac{\left(1+\xi_{s}\right) \cdot \xi_{s}}{2}
$$

$$
C_{2}^{1}=\frac{\left(1-2 . \xi_{s}\right)}{2} \quad C_{2}^{2}=2 . \xi_{s}^{2} \quad C_{2}^{3}=\frac{1}{2}
$$

$$
C_{2}^{1}=\frac{1}{2} \quad C_{2}^{2}=-1 \quad C_{2}^{3}=\frac{1}{2}
$$

Para obter a integral (4.156.a) basta utilizar (4.161) e multiplicar pela constante $\frac{\mathbf{1}}{\mathbf{D o}} \cdot \frac{\partial \mathbf{D}}{\partial \mathbf{n}}$. A integral (4.157.a) é obtida a partir de (4.162).

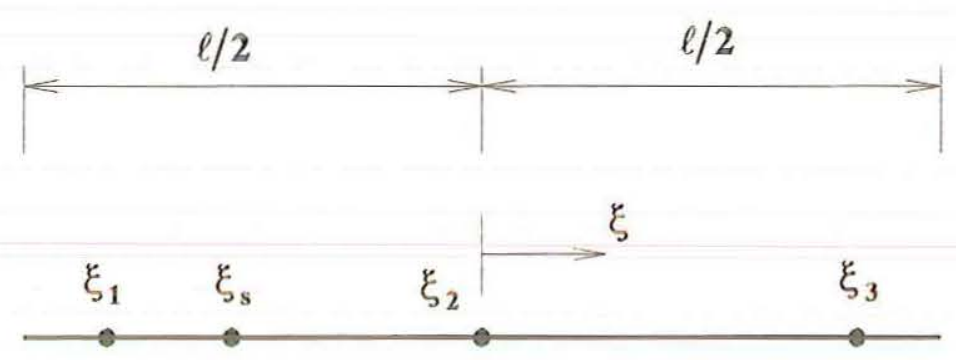

$$
\mathrm{a}=\ell . \phi_{2 \mathrm{~s}} \quad \mathrm{~b}=\ell \cdot \phi_{1 \mathrm{~s}}
$$

FIGURA 4.29 - Elemento de Contorno Linear Descontínuo.

A integral (4.156.b) tem também que ser resolvida analiticamente.

onde:

$$
\mathbf{D}(\mathrm{s})=\mathbf{a s} . \Gamma+\mathbf{b s}
$$




$$
M_{n}^{*}=-\frac{1}{4 \cdot \pi} \cdot[(1+v) \cdot \ln (r)+v]
$$

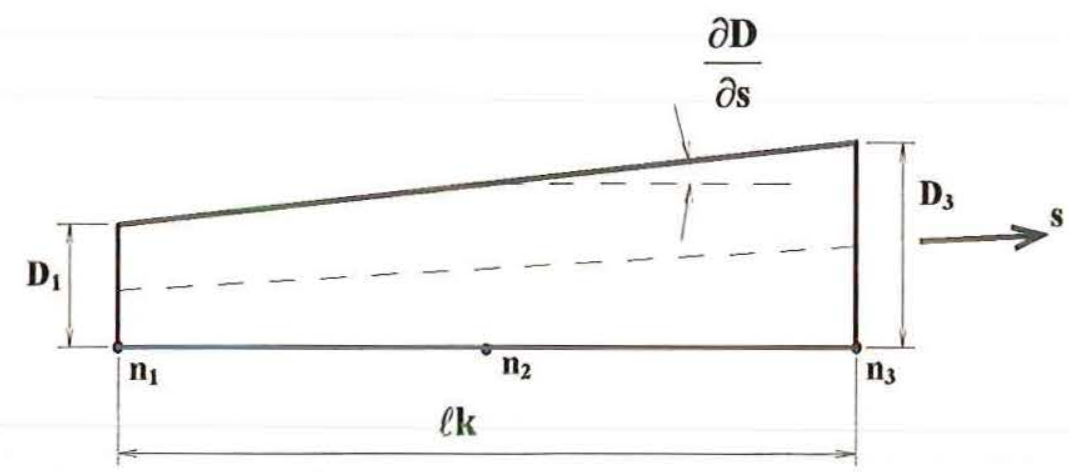

FIGURA 4.30 - Variação Linear da Rigidez no Contorno da Placa.

Da mesma maneira que foi feita a integração para obtenção de (4.160) a (4.163), aqui também será adotado um ponto de carregamento genérico com coordenada adimensional de $\xi_{s}$, para obtenção da integral (4.157). Aqui a aproximação é contínua para as variáveis.

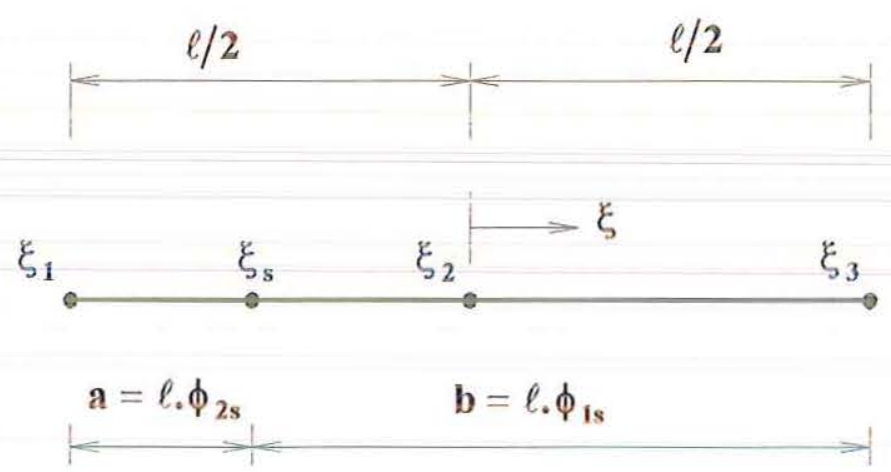

FIGURA 4.31 - Posição do Ponto Singular $\xi_{\mathrm{s}}$.

Escolhido um ponto genérico $\xi_{\mathrm{s}}$ como ponto singular, será necessário resolver três integrais referente a $\phi_{1}, \phi_{2}, \phi_{3}$. 
A integral definida (4.156.b) é uma integral imprópria, pois tem uma singularidade em um ponto, que neste caso específico é a posição zero, podendo então a integral (4.156.b) ser escrita como:

$$
\begin{aligned}
& \int_{\Gamma}\left(-\frac{D(s)}{D_{0}} \cdot M_{n}^{*}(Q, P)\right) \cdot \phi_{n}(P) \cdot d \Gamma(P) \\
& =\lim _{\varepsilon \rightarrow 0}\left(\int_{-a}^{-\varepsilon}\left(-\frac{D(s)}{D o} \cdot M_{n}^{*}(Q, P)\right) \cdot \phi_{n}^{a}(P) \cdot d \Gamma(P)+\int_{\varepsilon}^{b}\left(-\frac{D(s)}{D 0} \cdot M_{n}^{*}(Q, P)\right) \cdot \phi_{n}^{b}(P) \cdot d \Gamma(P)\right)
\end{aligned}
$$

Obtendo-se a integral (4.168) para $\phi_{1}$, será conveniente expressar as variáveis em função de r, figura 4.31. Será necessário também definir $\phi_{1}$ para os trechos a e b, respectivamente $\phi_{1}^{\mathrm{a}}$ e $\phi_{1}^{\mathrm{b}}$, CHAVES (1997).
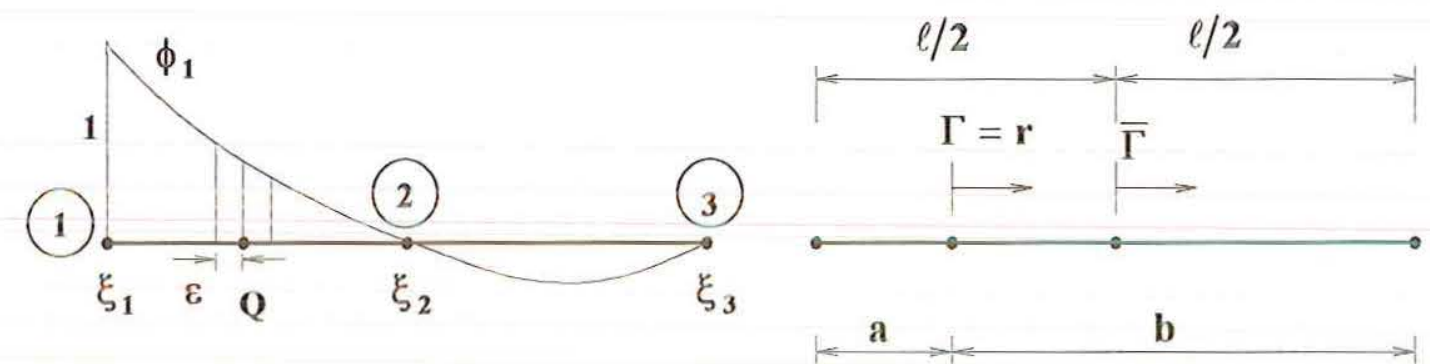

FIGURA 4.32 - Função de Forma $\phi_{1}$.

Trecho a

$$
\begin{aligned}
& \phi_{1}^{a}=\frac{1}{(a+b)^{2}} \cdot(-r-b) \cdot(-2 \cdot r+a-b) \\
& \Gamma=-r \Rightarrow d \Gamma=-d r \\
& D(s)=-a s \cdot r+b s
\end{aligned}
$$

Trecho b 


$$
\begin{aligned}
& \phi_{1}^{b}=\frac{1}{(a+b)^{2}} \cdot(r-b) \cdot(2 \cdot r+a-b) \\
& \Gamma=r \Rightarrow d \Gamma=d r \\
& D(s)=a s . r+b s
\end{aligned}
$$

Definidas as funções interpoladoras e variáveis envolvidas para os trechos a e b, pode-se resolver as integrais (4.168) para a função de forma $\phi_{1}$, resultando em:

$$
h_{1}^{\mathrm{n}}=\frac{-1}{288 \cdot \text { Do. }\left[\pi \cdot(\mathrm{a}+\mathrm{b})^{2}\right]} \cdot\left[\left(\mathrm{h}_{11}^{\mathrm{a} 1}+\mathrm{h}_{11}^{\mathrm{a} 2}\right) \cdot \mathrm{as}+\left(\mathrm{h}_{11}^{\mathrm{b} 1}+\mathrm{h}_{11}^{\mathrm{b} 2}\right) \cdot \mathrm{bs}\right]
$$

onde:

$$
\begin{aligned}
\begin{aligned}
h_{11}^{\mathrm{a} 1}=\left[1 2 \cdot \mathrm { a } ^ { 2 } \cdot \left(\mathrm{a}^{2}+3 \cdot a \cdot b\right.\right. & \left.+3 \cdot b^{2}\right) \cdot \ln (a)+18 \cdot a^{2} \cdot b^{2}+11 \cdot a^{4}+30 \cdot a^{3} \cdot b+ \\
& \left.+12 \cdot b^{3} \cdot a \cdot \ln (b)+2 \cdot b^{3} \cdot a+3 \cdot b^{4}\right] \cdot(1+v)
\end{aligned} \\
\begin{aligned}
& h_{11}^{\mathrm{a} 2}=-12 \cdot a \cdot(a+b)^{3} \\
& h_{11}^{\mathrm{b} 1}=\left\{\left[12 \cdot \mathrm{b}^{2} \cdot(3 \cdot a-b) \cdot \ln (b)-12 \cdot a \cdot\left(3 \cdot b \cdot a+a^{2}+6 \cdot b^{2}\right) \cdot \ln (a)-54 \cdot b \cdot a^{2}+\right.\right. \\
&\left.\left.+22 \cdot b^{3}-14 \cdot a^{3}-18 \cdot a \cdot b^{2}\right] \cdot(1+v)\right\}
\end{aligned}
\end{aligned}
$$

$$
h_{11}^{b 2}=12,(a+b)^{3}
$$

Analogamente a obtenção da integral (4.168) para a função de forma $\phi_{1}$, pode-se obter a integral para a função $\phi_{2}$, figura 4.32 . 

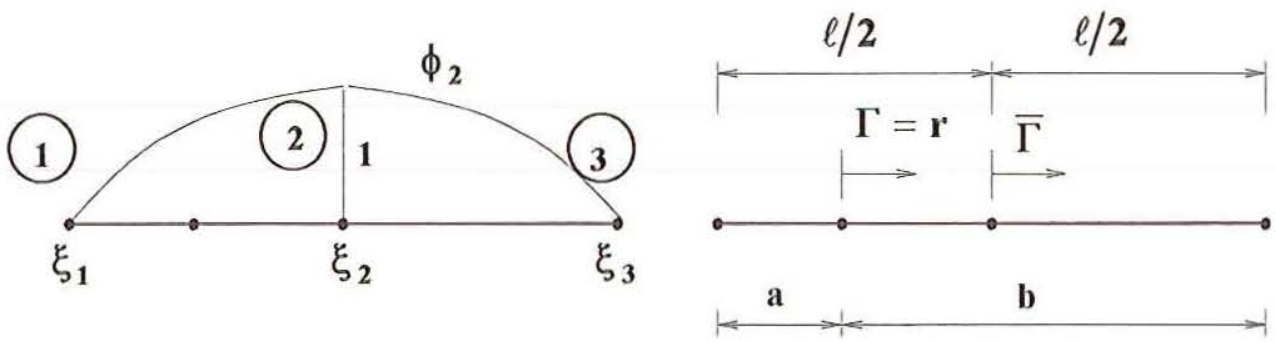

FIGURA 4.33 - Função de Forma $\phi_{2}$.

Trecho a

$$
\begin{aligned}
& \phi_{2}^{a}=1-\left(\frac{-2 \cdot r+a-b}{a+b}\right)^{2} \\
& d \Gamma=-d r \\
& D(s)=-a s . r+b s
\end{aligned}
$$

Trecho b

$$
\begin{aligned}
& \phi_{2}^{b}=1-\left(\frac{2 \cdot r+a-b}{a+b}\right)^{2} \\
& d \Gamma=d r \\
& D(s)=-a s \cdot r+b s
\end{aligned}
$$

Definidas as funções interpoladoras e variáveis envolvidas para os trechos a e b, pode-se resolver as integrais (4.168) para a função de forma $\phi_{2}$, resultando em:

$$
h_{2}^{\mathrm{n}}=\frac{1}{144 . \text { Do }\left[\pi \cdot(\mathrm{a}+\mathrm{b})^{2}\right]} \cdot\left[\left(\mathrm{h}_{13}^{\mathrm{a} 1}+\mathrm{h}_{13}^{\mathrm{a} 2}\right) \cdot \mathrm{as}+\left(\mathrm{h}_{13}^{\mathrm{b} 1}+h_{13}^{\mathrm{b} 2}\right) \cdot \mathrm{bs}\right]
$$


onde:

$$
\begin{aligned}
h_{13}^{\mathrm{a} 1}=\left[\left(24 \cdot b^{3} \cdot a+12 \cdot b^{4}\right) \cdot \ln (b)\right. & +\left(-12 \cdot a^{4}-24 \cdot a^{3} \cdot b\right) \cdot \ln (a)+4 \cdot b^{3} \cdot a-5 \cdot a^{4}+ \\
& \left.+5 \cdot b^{4}-4 \cdot a^{3} \cdot b\right] \cdot(1+v) \\
h_{13}^{\mathrm{a} 2}=12 \cdot(a-b) \cdot(a+b)^{3} & \\
h_{13}^{b 1}= & {\left[24 \cdot(a+3 \cdot b) \cdot a^{2} \cdot \ln (a)+24 \cdot(3 \cdot a+b) \cdot b^{2} \cdot \ln (b)+4 \cdot(-9 \cdot b+a) \cdot a^{2}+\right.}
\end{aligned}
$$

$$
\left.+4 \cdot(-9 \cdot a+b) \cdot b^{2}\right] \cdot(1+v)
$$

$$
h_{13}^{\mathrm{b} 2}=-24 \cdot(\mathbf{a}+\mathbf{b})^{3}
$$

Analogamente a obtenção da integral (4.168) para a função de forma $\phi_{1}$ e $\dot{\varphi}_{2}$, pode-se obier a integral para a função $\dot{\phi}_{3}$, figura 4.34 .

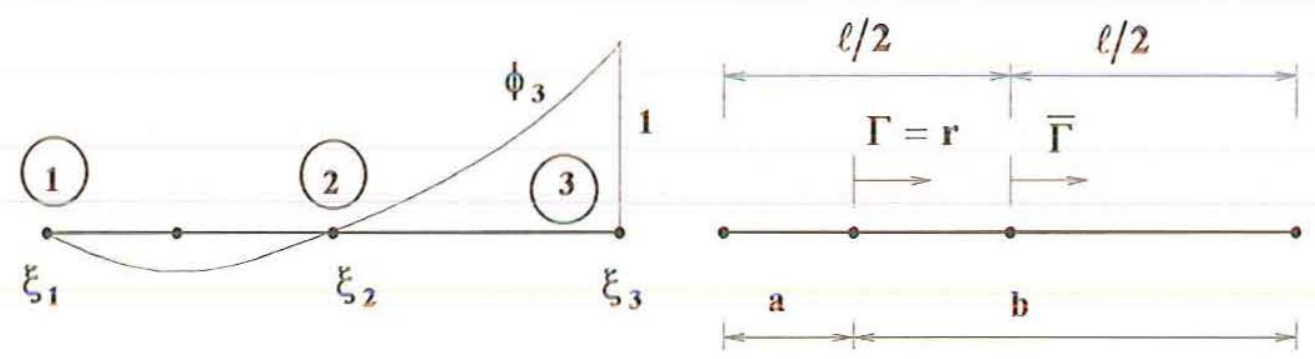

FIGURA 4.34 - Função de Forma $\phi_{3}$. 
Trecho a

$$
\begin{aligned}
& \phi_{3}^{a}=\frac{1}{(a+b)^{2}} \cdot(a-r) \cdot(-2 \cdot r+a-b) \\
& d \Gamma=-d r \\
& D(s)=-a s \cdot r+b s
\end{aligned}
$$

Trecho b

$$
\begin{aligned}
& \phi_{3}^{b}=\frac{1}{(a+b)^{2}} \cdot(a+r) \cdot(2 \cdot r+a-b) \\
& d \Gamma=d r \\
& D(s)=a s \cdot r+b s
\end{aligned}
$$

Definidas as funções interpoladoras e variáveis envolvidas para os trechos a $\mathrm{e}$ b, pode-se então resolver as integrais (4.168) para a função de forma $\phi_{3}$, resultando em:

$$
\mathbf{h}_{3}^{\mathrm{n}}=\frac{1}{288 \cdot \mathrm{Do} \cdot\left[\pi \cdot(\mathrm{a}+\mathrm{b})^{2}\right]} \cdot\left[\left(\mathbf{h}_{16}^{\mathrm{a} 1}+\mathbf{h}_{16}^{\mathrm{a} 2}\right) \cdot \mathbf{a s}+\left(\mathbf{h}_{16}^{\mathrm{b} 1}+\mathbf{h}_{16}^{\mathrm{b} 2}\right) \cdot \mathrm{bs}\right]
$$

onde:

$$
\begin{aligned}
& h_{16}^{a 1}=\left[-12 \cdot b^{2} \cdot\left(3 \cdot a \cdot b+b^{2}+3 \cdot a^{2}\right) \cdot \ln (b)-12 \cdot a^{3} \cdot b \cdot \ln (a)-\right. \\
& \left.-(a+b) \cdot\left(3 \cdot a^{3}-a^{2} \cdot b+19 \cdot a \cdot b^{2}+11 \cdot b^{3}\right)\right] \cdot(1+v)
\end{aligned}
$$

$$
h_{16}^{\mathrm{a} 2}=12 \cdot \mathrm{b} \cdot(\mathrm{a}+\mathrm{b})^{3}
$$

$$
\begin{aligned}
h_{16}^{b 1}=\left[-12 \cdot a^{2} \cdot(\right. & -3 \cdot b+a) \cdot \ln (a)-12 \cdot b \cdot\left(b^{2}+6 \cdot a^{2}+3 \cdot a \cdot b\right) \cdot \ln (b)-54 \cdot a \cdot b^{2}- \\
& \left.-18 \cdot a^{2} \cdot b-14 \cdot b^{3}+22 \cdot a^{3}\right] \cdot(1+v)
\end{aligned}
$$

$$
h_{16}^{b 2}=12 .(a+b)^{3}
$$




\subsection{Representações Algébricas Alternativas}

A representação do teorema de Betti para um ponto de um sólido considerando a variação de espessura dada pela eq.(3.26), pode se expressa também da seguinte maneira:

$$
\mathrm{m}_{\mathrm{ij}} \cdot w_{\mathrm{ij}^{*}}=\mathrm{m}_{\mathrm{ij}}^{*} \cdot w_{\mathrm{ij}_{\mathrm{j}}}+\frac{\Delta \mathrm{D}(\mathrm{s})}{\mathrm{D}_{\mathrm{o}}} \cdot \mathrm{m}_{\mathrm{ij}}^{*} \cdot w_{\mathrm{ij}_{\mathrm{j}}}
$$

Integrando-se no domínio a eq.(4.190), resulta:

$$
\int_{\Omega} \mathrm{m}_{\mathrm{ij}} \cdot w_{\mathrm{ij}_{\mathrm{j}}} \cdot \mathrm{d} \Omega=\int_{\Omega} \mathrm{m}_{\mathrm{ij}}^{*} \cdot w_{\mathrm{ij}_{\mathrm{j}}} \cdot \mathrm{d} \Omega+\int_{\Omega} \frac{\Delta \mathrm{D}(\mathrm{s})}{\mathrm{D}_{\mathrm{o}}} \cdot \mathrm{m}_{\mathrm{ij}}{ }_{\mathrm{ij}} \cdot w_{9 \mathrm{ij}} \cdot \mathrm{d} \Omega
$$

Podendo ter três possibilidades para resolução do problema:

a) sem efetuar integração por partes e através da inversa da lei de Hooke, substituindo-se na terceira integral de domínio $\mathrm{em}_{\mathrm{ij}}$;

b) fazendo-se uma integração por partes, resulta numa integral alternativa da rotação $\theta$;

c) efetuando-se duas integrações por partes, e usando a lei de Hooke, resulta: $w_{9_{\mathbf{i j}}} \rightarrow w$.

Desenvolvendo-se os dois membros desta equação, através da integração pr partes, obtém-se a equação integral de momento de um ponto q do domínio

Considerando-se a primeira por exemplo, percebe-se que é idêntica a equação de momento inicial CHUEIRI (1994). Neste caso depois da discretização as equações de contorno e de momento tem-se:

$$
\text { H. U }=\mathbf{G} \cdot \mathbf{P}+\mathbf{F}+\mathbf{E} \cdot \mathbf{M}^{\mathbf{o}}
$$


e

$$
\mathbf{M}=-\mathbf{H}^{\prime} \cdot \mathbf{U}+\mathbf{G}^{\mathbf{\prime}} \cdot \mathbf{P}+\mathbf{E}^{\mathbf{\prime}} \cdot \mathbf{M}^{\mathbf{0}}+\mathbf{F}
$$

Após as simplificações usuais e substituindo na equação de momento, CHUEIRI (1994), obtêm-se:

$$
\begin{aligned}
\mathbf{M}^{\mathbf{v}} & =\mathbf{N}+\mathbf{S} \cdot \mathbf{M}^{\mathbf{o}} \\
\mathbf{M}^{\mathbf{e}} & =\mathbf{N}+\mathbf{S}^{\mathbf{1}} \cdot \mathbf{M}^{\mathbf{o}}
\end{aligned}
$$

considerando-se que no problema elástico com variação de rigidez, pode-se expressar $\mathbf{M}^{\mathbf{v}}$ em função de $\mathbf{M}^{\mathbf{0}}$, figura 4.34:

$$
M^{0}=\left(\frac{D_{0}}{D}-1\right) \cdot M^{v}=T \cdot M^{v}
$$

chegando-se a:

$$
\left(\mathbf{I}-\mathbf{S}^{\prime} \cdot \mathbf{T}\right) \cdot \mathbf{M}^{\mathbf{v}}=\mathbf{N}
$$

ou ainda :

$$
M^{\mathrm{v}}=\left(\mathbf{I}-\mathrm{S}^{\prime} \cdot \mathrm{T}\right)^{-1} \cdot \mathrm{N}
$$

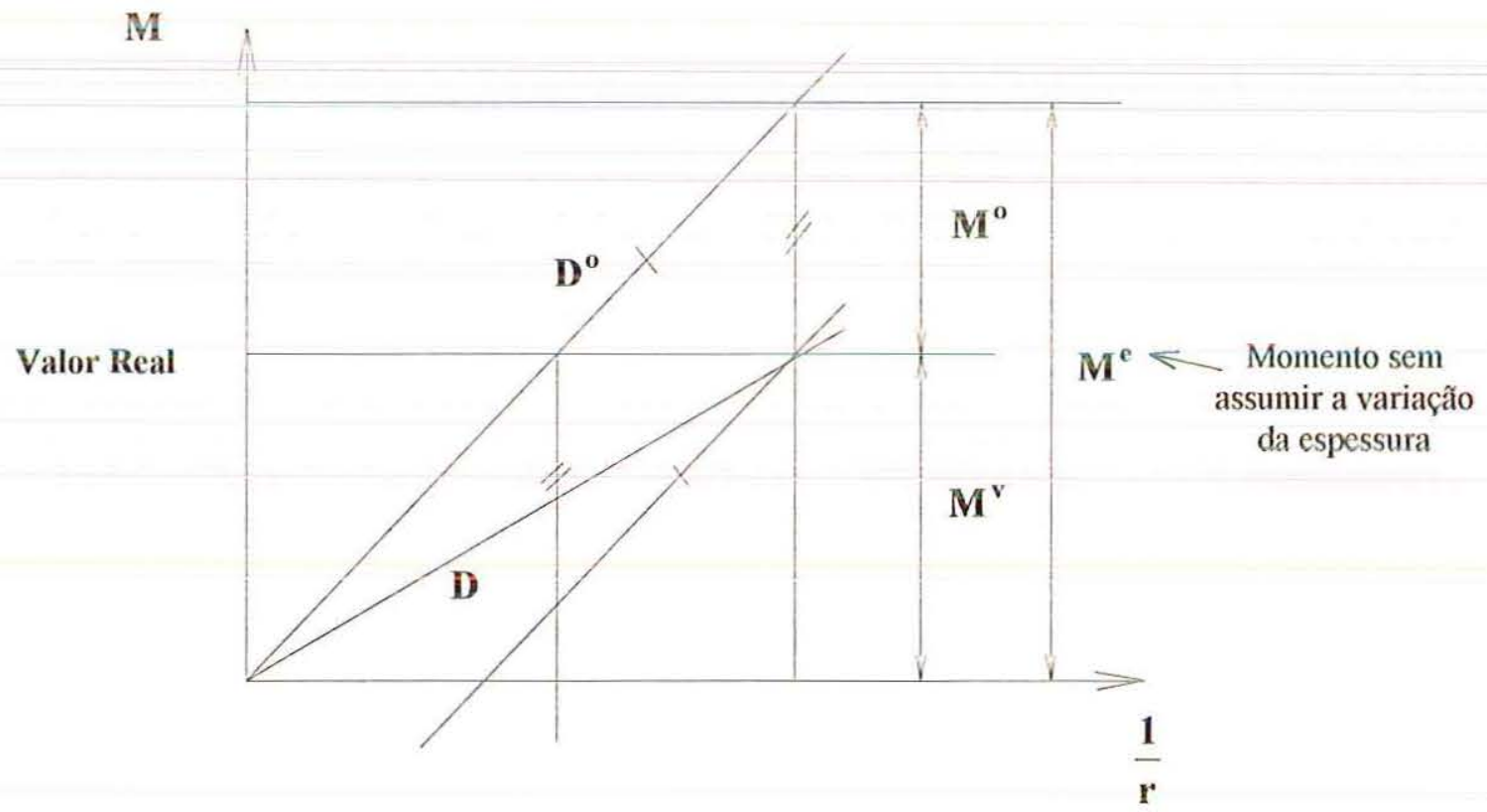

FIGURA 4.35 - Momento Elástico e Momento Inicial. 


\section{RESULTADOS E CONCLUSÃO}

\subsection{Aplicação Numérica}

São apresentado, a seguir, alguns exemplos de aplicação da formulação desenvolvida na análise de placas com espessura constante e variável sendo estes valores comparados com o método dos elementos finitos. O elemento finito de placa utilizado foi o DKT (Discrete Kichhoff Theory), utilizado pelo autor em CHAVES (1996), programa de placas para simular comportamento de pavimentos de edifício. Neste programa também é possível utilizar elemento finito de placa quadrangular (DKQ), obtido a partir da condensação estática de quatro elementos finitos triangulares DKT.

Em todos os exemplos apresentados, utiliza-se o coeficiente $a=0,25$, que define o posicionamento dos pontos de carregamento conforme a equação (4.58). Nos elementos de contomo com nós duplos, as equações são descritas para $\xi_{\mathrm{s}}= \pm 0,6$, embora a variação de $\xi_{\mathrm{s}}$ no intervalo de $| \pm 0,4| \leq\left|\xi_{\mathrm{s}}\right| \leq| \pm 0,6|$ possa ser utilizada sem perda de precisão CHUEIRI (1994).

\subsubsection{Exemplo 1.}

Neste exemplo será processada uma placa quadrada, com espessura (t) constante, com dois lados opostos apoiados e outros dois engastados, com carregamento uniformemente distribuído. Adotam-se ainda os seguintes valores:

- $\mathbf{E}=1.092,00$; 
- $v=\mathbf{0 , 3}$;

- $\mathbf{q}=1,0$;

- $\mathbf{a}=1,0$;

- espessura da placa : $\mathbf{t}=\mathbf{0 , 1 0}$.
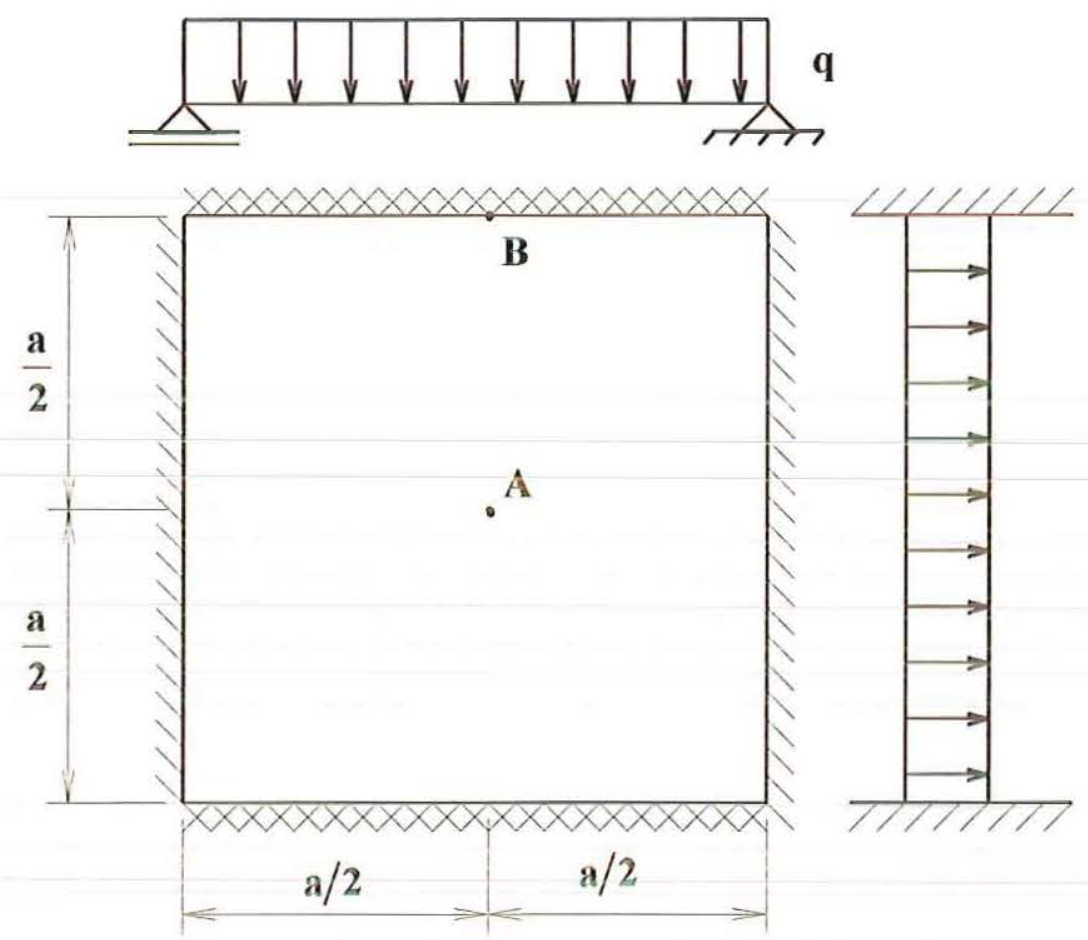

FIGURA 5.1 - Placa Quadrada, de Espessura Constante, Apoiada em Dois Lados Opostos e Engastada nos Outros Dois.

Foi analisada através de diversas malhas de discretização da placa da figura 5.1, tanto com elemento finitos quanto elementos de contorno. A discretização da malha de elementos finitos foi uniforme. As características da discretização via método dos elementos de contorno e via método dos elementos finitos estão apresentadas nas tabelas 5.1 e 5.2 , respectivamente. Em seguida apresentam-se os gráficos comparativos entre os dois métodos empregados, de deslocamentos do ponto A, figura 5.1, e de momentos nos pontos $\mathrm{A}$. 
TABELA 5.1 - Discretização Via Elemento de Contorno - Placa de Espessura Constante.

\begin{tabular}{|c|c|c|}
\hline \multicolumn{3}{|c|}{ DISCRETIZAÇÃo } \\
(ELEMENTO DE CONTORNO) \\
\hline $\begin{array}{c}\text { Número de } \\
\text { elementos de } \\
\text { contorno }\end{array}$ & Número de cantos & $\begin{array}{c}\text { Número de Graus } \\
\text { de Liberdade }\end{array}$ \\
\hline 4 & 4 & 28 \\
\hline 8 & 4 & 44 \\
\hline 16 & 4 & 76 \\
\hline 32 & 4 & 140 \\
\hline
\end{tabular}

TABELA 5.2 - Discretização Via Elementos Finitos.

\begin{tabular}{|c|c|c|}
\hline \multicolumn{3}{|c|}{ DISCRETIZACÃO } \\
(ELEMENTOS FINITOS) \\
\hline $\begin{array}{c}\text { Número de } \\
\text { elementos finitos } \\
\text { DKQ }\end{array}$ & $\begin{array}{c}\text { Número de Graus } \\
\text { de Liberdade }\end{array}$ & $\begin{array}{c}\text { Equivalência do } \\
\text { número de Graus } \\
\text { de Liberdade para } \\
\text { o DKT }\end{array}$ \\
\hline 4 & 27 & 39 \\
\hline 9 & 48 & 75 \\
\hline 16 & 75 & 123 \\
\hline 64 & 363 & 635 \\
\hline 100 & 243 & 663 \\
\hline
\end{tabular}




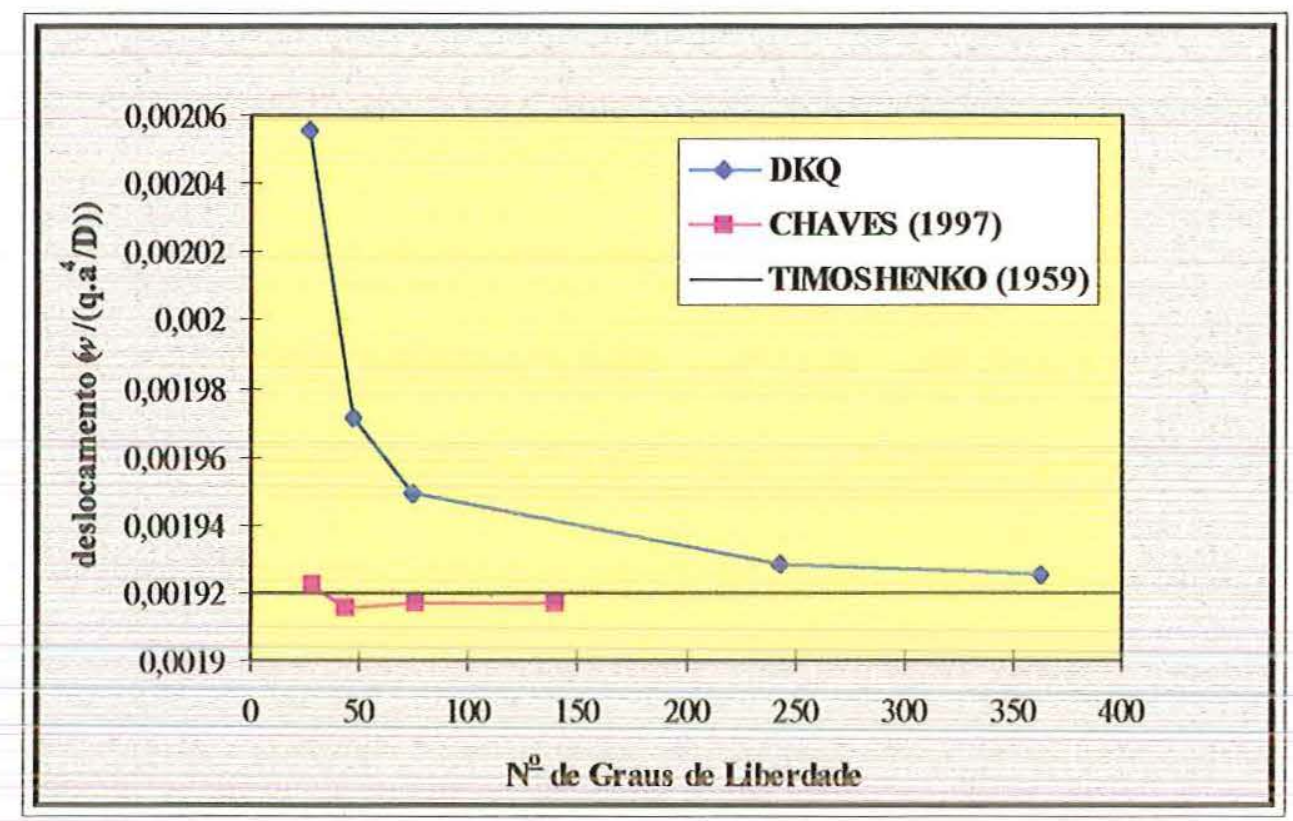

GRÁfICO 5.1 - Deslocamento no Ponto A.

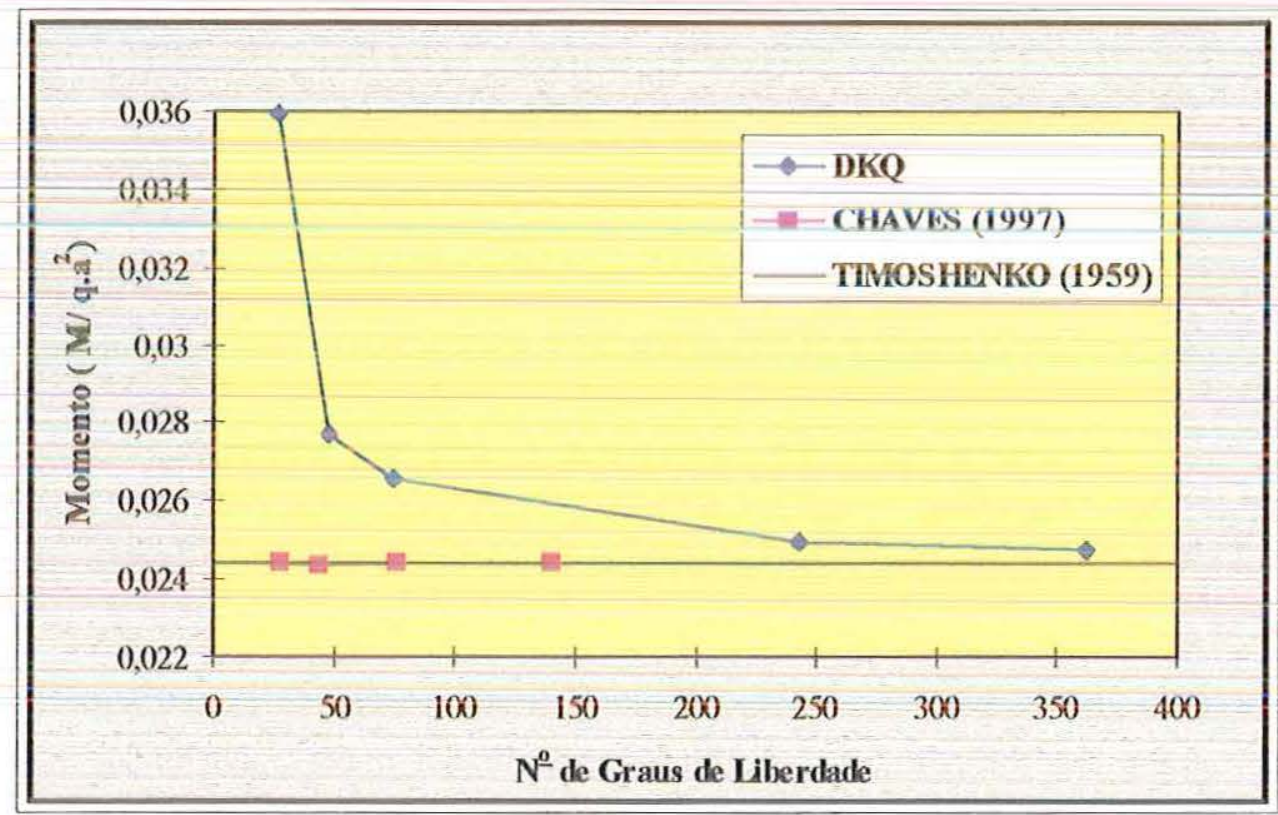

GRÁFICO 5.2 - Momento $\mathbf{M}_{\mathbf{x}}$ no Ponto $\mathbf{A}$. 


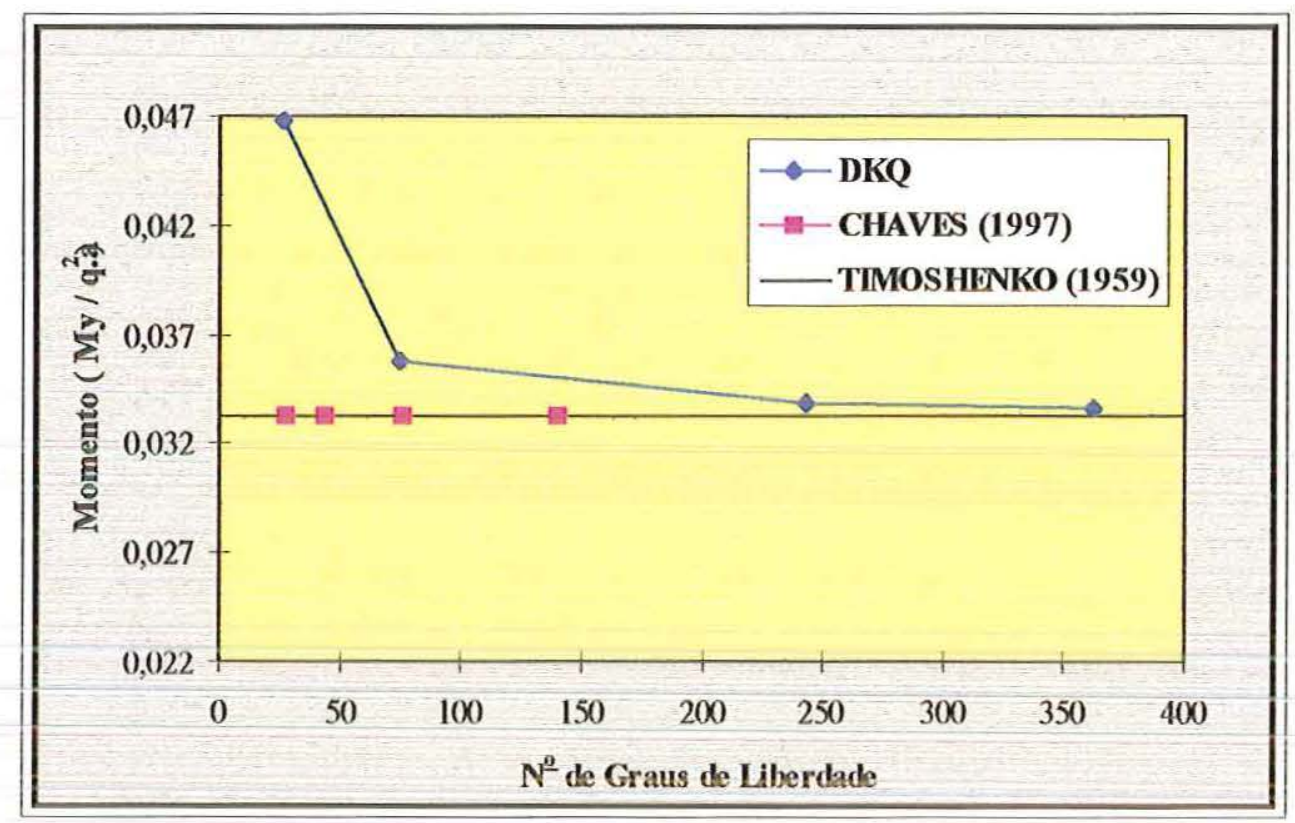

GRÁfICO 5.3 - Momento $\mathbf{M}_{\mathbf{y}}$ no Ponto A.

\subsubsection{Exemplo 2.}

Neste exemplo será processada uma placa quadrada, com espessura variável, com dois lados opostos apoiados e outros dois livres, com carregamento uniformemente distribuído. Considerando-se ainda os seguintes valores:

- $\mathrm{E}=1.092,00$

- $v=0,3$;

- $q=1,0$;

- $\mathbf{a}=\mathbf{1}, 0$;

A discretização de elementos finitos utilizada foi a mesma adotada no exemplo 1, mas para a discretização em elementos de contorno foi também utilizadas células internas acarretando nos pontos internos incógnitas de deslocamentos. Aumentandose assim o número de graus de liberdade, tabela 5.3. 
TABELA 5.3 - Discretização Via Elemento de Contorno - Placa Espessura Variável.

\section{DISCRETIZAÇÃO (ELEMENTO DE CONTORNO)}

\begin{tabular}{|c|c|c|}
\hline $\begin{array}{c}\text { Número de } \\
\text { elementos de } \\
\text { contorno }\end{array}$ & Número de cantos & $\begin{array}{c}\text { Número de Graus } \\
\text { de Liberdade }\end{array}$ \\
\hline 4 & 4 & 29 \\
\hline 8 & 4 & 53 \\
\hline 16 & 4 & 125 \\
\hline 32 & 4 & 365 \\
\hline
\end{tabular}

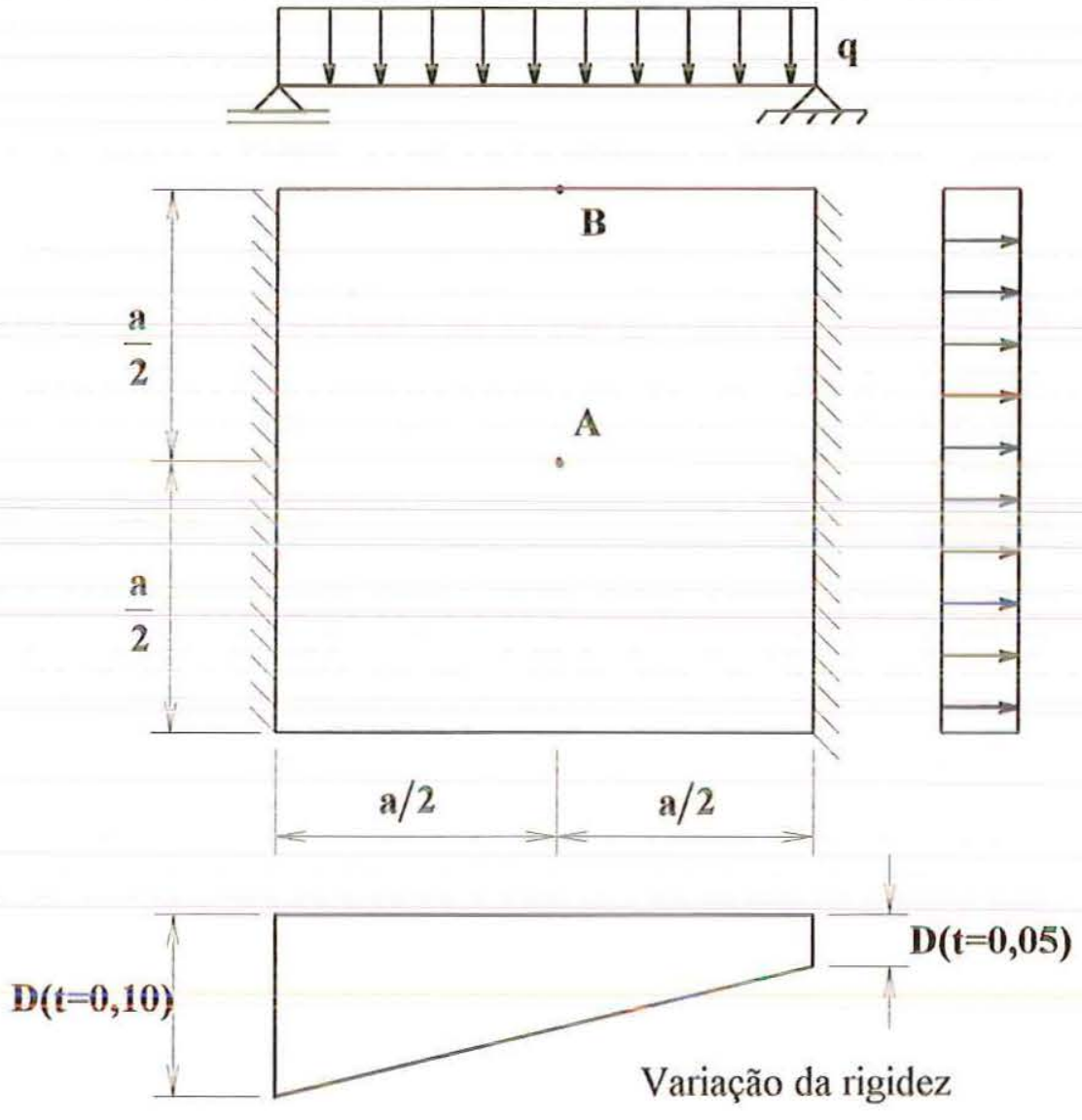

FIGURA 5.2 - Placa Quadrada Apoiada em Dois Lados Opostos e Livre nos Outros Dois. 


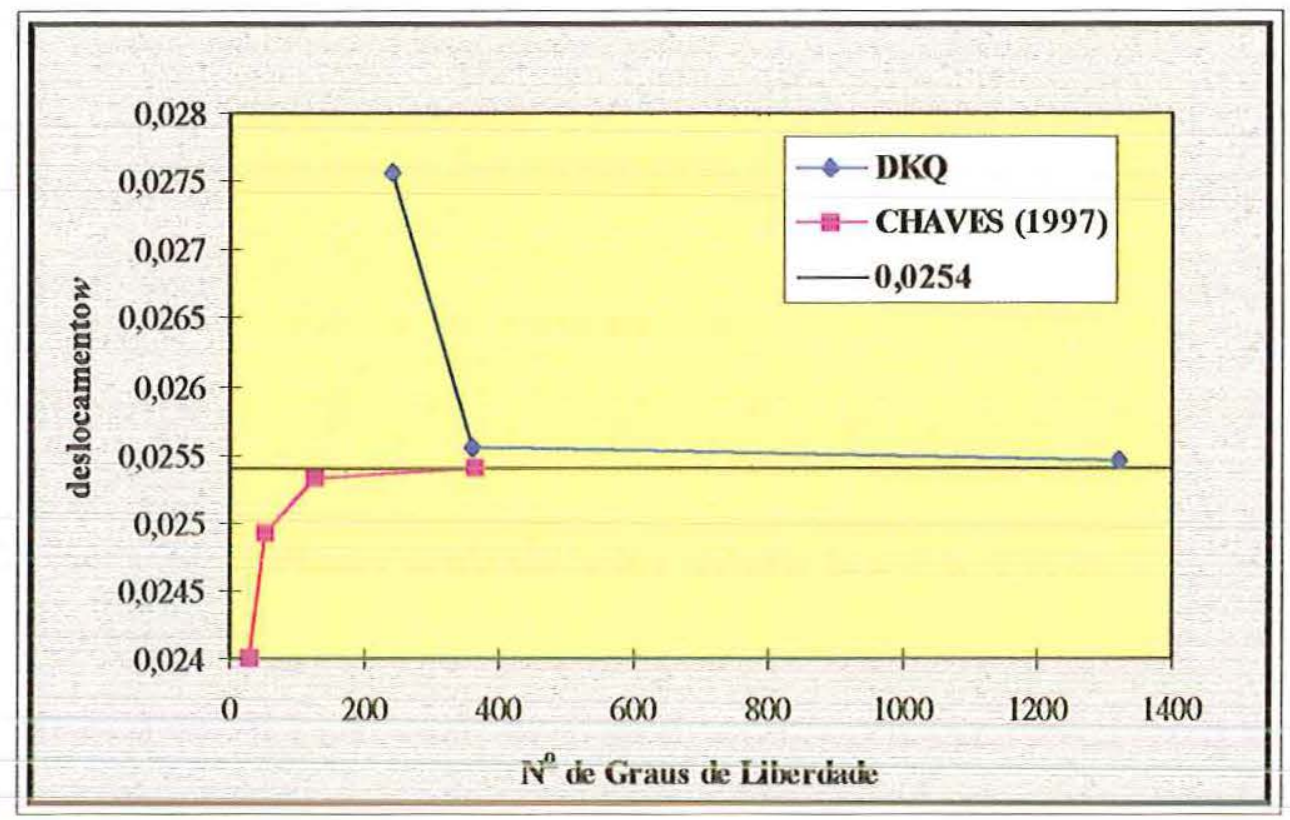

GRÁFICO 5.4 - Deslocamento no Ponto A.

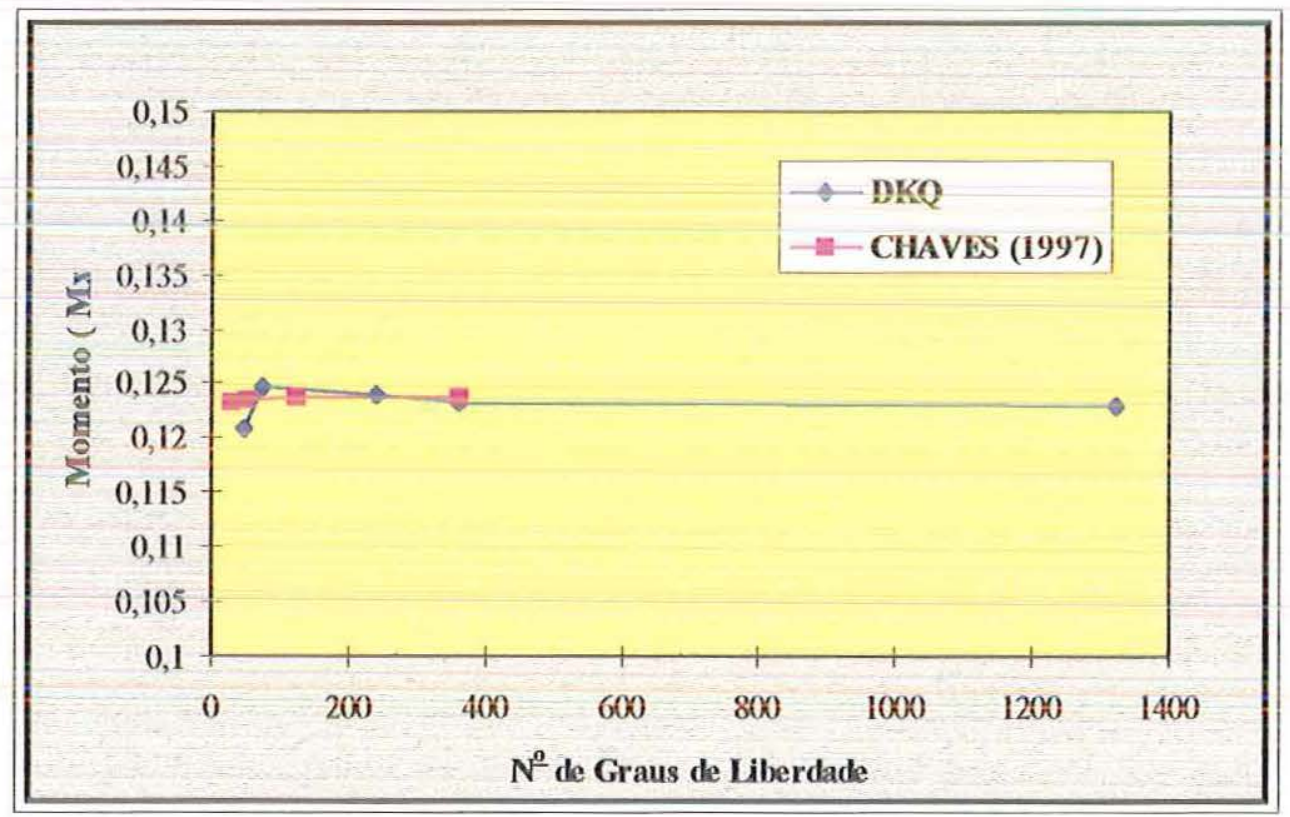

GRÁFICO 5.5 - Momento Mx no Ponto A. 


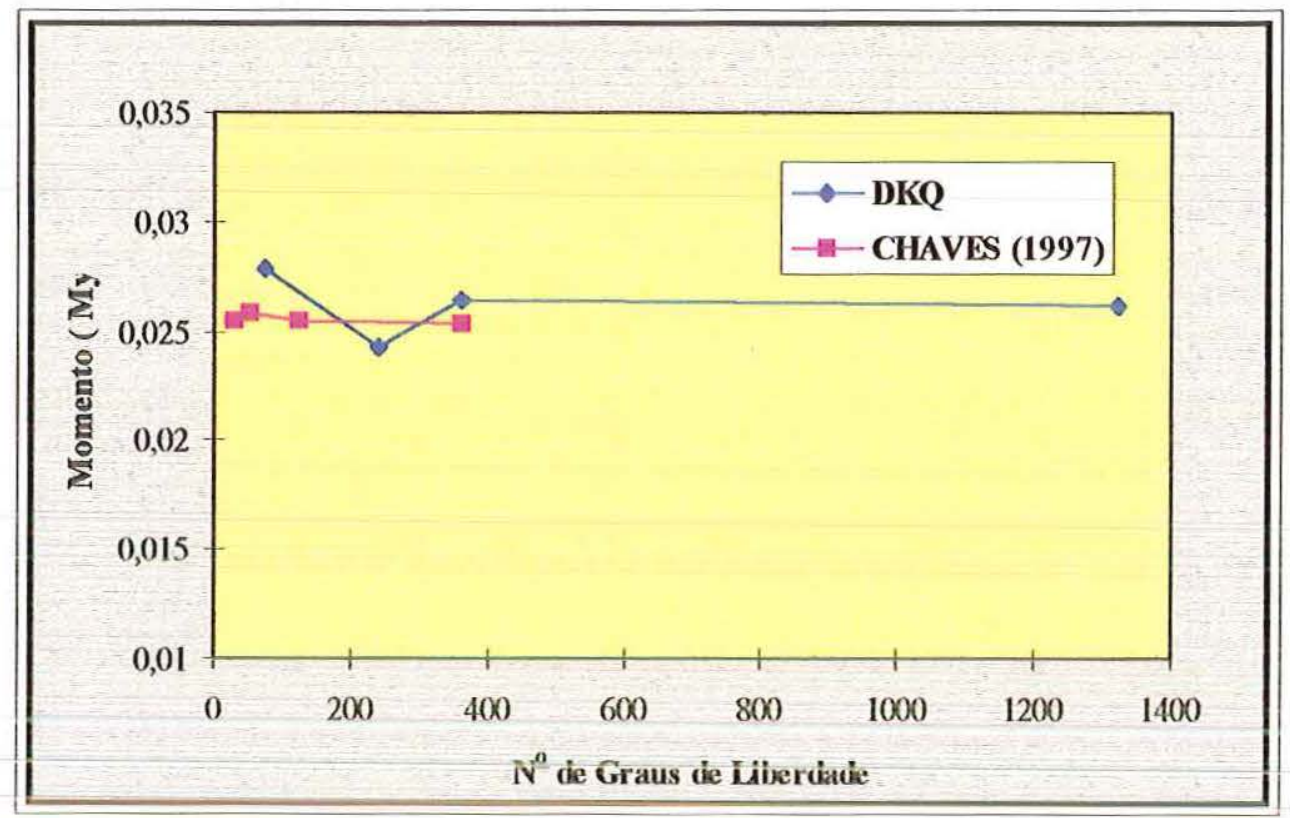

GRÁFICO 5.6 - Momento My no Ponto A. 


\subsection{Conclusão}

Neste trabalho foi desenvolvido uma formulação do Método dos Elementos de Contorno para consideração da variação da espessura de placas, utilizando-se a teoria clássica de Kirchhoff. A formulação proposta discute os vários caminhos para o tratamento do problema. Em particular, foi desenvolvido e elaborado um programa correspondente para a formulação que trata como incógnitas internas os deslocamentos transversais. Esta formulação mostrou-se eficiente para a solução elástica de placas.

A precisão dos resultados obtidos comprova a viabilidade da utilização do Método dos Elementos de Contorno nesse tipo de análise; destaca-se, como vantagem desse método em relação a outras técnicas numéricas. Por exemplo elementos finitos não se pode eliminar descontinuidade de espessura para poder simular a variação de espessura o que não acontece em elementos de contorno. Observou-se também que o termo de domínio não requer uma grande discretização. Apenas um grau de liberdade está associado a pontos internos, o que reduz a importância dessa iniegral. Formulações que apresentam mais graus de liberdade em pontos internos certamente necessitarão de maior discretização.

A formulação adotada, considerando a rigidez variável, possibilitará a análise não-linear de placas considerando método de Newton-Raphson. Com esse procedimento a perda da rigidez da placa devido a plastificação ou outro efeito nãolinear que pode ser facilmente incorporada. 


\section{BIBLIOGRAFICA}

ABDEL-AKHER, A. ; HARTLEY, G.A. (1988). Analytic integration procedure for plate bending analysis. In: BREBBIA,C.A. ed.. Boundary elements X. C. M. Publ., v.3, p.391-405.

ABDEL-AKHER, A. ; HARTLEY, G.A. (1989a). Evaluation of boundary integrals for plate bending. Int. J. Num. Meth. Eng., v.28, n.2, p.75-93.

ABDEL-AKHER, A. ; HARTLEY, G.A. (1989b). Boundary integration and interpolation procedures for plate bending. Int. J. Num. Meth. Eng., v.28, n.6, p.1389-1408.

ABEL, N.H. (1881). Ouevres complètes. Norvegieu Christiania, 1.

AKKARI, M.M. ; MUTCHINSON, J.R. (1988). Boundary point collocation method for the vibrations of a Mindin thick Plate. In: BREBBIÁ,C.A. ed.. Boundary elements X: vol.3 Stress Analysis, p.465-476.

ALTIERO, N.J. ; SIKARSKIE, D.L. (1978). A boundary integral method applied to plates of arbitrary plan form. Computers \& Structures, v.9, p.163-168.

ANDERSSEN, R.S. et alii. (1980). The application and numerical solution of integral equations. Alpheu aan deu Rijn, The Netherlands, Sijthoff and Noordhoff.

ARGYRIS, J.H. ; KELSEY, S. (1960). Energy theorems and structural analysis. London, Butteerworths. 
BANERJEE, P.K. (1978). Analysis of axially and laterally loaded pile groups In: SCOTT, C.R., eds. Developments in soil mechanics-1. Barking appl. Sci. Publ., p.317-346.

BANERJEE, P.K. ; DRISCOLL, R.M. (1976). Three-dimensional analysis of raked pile groups. Proc. Inst. Civ. Eng., n.61, p.653-671.

BARCELLOS, C.S. ; SILVA, L.H.M. (1987). A boundary element formulation for the Mindlin's plate model. In: BREBBIA,C.A.;VENTURINI,W.S. eds. Boundary element tecniques: applications in stress analysis and heat transfer. Southampton, C.M. Publ.

BARRETTO, S.F.A. (1995). Análise elastodinâmica de placas, através do método dos elementos de contorno, com inieração solo-estritîtura. São Carlos. $115 \mathrm{p}$. Tese (Doutorado) - Escola de Engenharia de São Carlos, Universidade de São Paulo.

BEER, G.; WATSON, J.O. (1992). Introduction to finite and boundary element methods for engineers. England. John Wiley \& Sons Ltd.

BETTI, E. (1872). Teoria dell elasticità. II Nuovo cimiento, ser.3 v.6-10.

BEZINE, G. (1978a). Boundary integral formulation for plate flexure with arbitrary boundary conditions. Mechanics Research Communications, v.5, n.4, p.197-206.

BEZINE, G. ; GAMBY, D.A. (1978b). A new integral equation formulation for plate bending problems. In: BREBBIA, C.A. ed. Recent advances in boundary element methods, p.327-342. London, Pentech Press.

BEZINE, G. P. (1988). A nem BEM for bending of plates on elastic foundations. Int. J. Solids and Siruciures, v.24, n.6, p.557-565.

BEZINE, G.P. ; CIMETIERRE, A. ; GELBERT, J.P. (1985). Unilateral buckling of thin elastic plates by the boundary integral equation method. Int. J. Num. Meth. Eng., v.21, p.2189-2199.

BREBBIA, C.A. (1978). The boundary element method for engineers. London. Pentech Press. 
BREBBIA, C.A.; DOMINGUEZ, J. (1989). Boundary elements: an introductory course. Southampton. New York, CMP/McGraw-Hill.

BUZAR, M.A.R. (1996). Análise de placas com ométodo dos elementos finitos e de contorno na modelagem de um edificio. 112p. Brasília, DF. Dissertação (Mestrado). Universidade de Brasília.

CHAVES, E.W.V. (1996). SRP - sistema de resolução de pavimentos pelo método dos elementos finitos. São Carlos. Departamento de Engenharia de Estruturas Escola de Engenharia de São Carlos.

CHAVES, E.W.V. (1997). PLANBEM97 - Análise de placas através do método dos elementos de contorno. São Carlos - Departamento de Engenharia de Estruturas Escola de Engenharia de São Carlos, Universidade de São Paulo.

CHUEIRI, L.H.M. (1994). Formulação do método dos elementos de contorno para análise elasioplásíca de placas. São Carlos. 219p. Tese (Doutorado) - Escola de Engenharia de São Carlos, Universidade de São Paulo.

CLOUGH, R. W. (1960). The finite element method, in plane stress analysis. $2^{\text {nd }}$ ASCE Conference On Eletronic Computation, Pittsburgh, USA, September. Proceedings.

CLOUGH, R.W. (1990). Original formulation of the finite element method. J. Finite Elem. Anal. Des., v. 7, n. 2 , p.89-101.

COSTA JR., J.A. ; BREBBIA, C.A. (1985). Elastic buckling of plates using the boundary element method. In: BREBBIA,C.A.; MAIER,G. ed. Boundary element VII. Proc. 7th. Int. Conf. Berlin, Springer-Verlag.

COSTA JR. , J.A. ; BREBBIA, C.A. (1985). Bending of plates on elastic foundations using the boundary element method. In: INTERNATIONAL CONFERENCE, $2^{\text {nd }}$, Southampton, 1985- Proceedings.: Berlin, Springer-Verlag.

COSTA JR. , J.A. ; BREBBIA, C.A. (1985). The boundary element method applied to plates on elastic foundations. Engineering Analysis, v.2, n.4, p.174-183. 
COSTA JR. , J.A. ; BREBBIA, C.A. (1986). On the reduction of domain integrals to the boundary for the BEM formulation of plates on elastic foundations. Engineering Analysis, v.3, n.2, p.123-126.

CROUCH, S.L.; STARFIELD, A.M. (1983). Boundary element methods in solid mechanics. London. Geoge Allen \& Unwin Ltd.

CRUSE, T.A. (1968). A direct formulation and numerical solution of the general transient elastodynamics problem II . Journal. Math. Analysis. Applications, v. 22, p. $341-355$

CRUSE, T.A. (1969). Numerical Solutions in Three dimensional elastostatics. Int. J. Solids and Structures, v.5, p.1259-1274.

CRUSE, T.A. (1973). Application of the boundary-integral equation method to threedimensional stress analysis. Computers \& Structures, v.3, p.509-527.

CRUSE, T.A. (1974). An improved boundary-integral equation method for three dimensional elastic stress analysis. Computers \& Siructures, v.4, p.741- 754.

CRUSE, T.A. ; RIZZO F.J. (1968). A direct formulation and numerical solution of the general transient elastodynamics problem I. Journal Math. Analysis Applications, v.22, p.244-259.

CRUSE, T.A.; VANBUREN, W. (1971). Three dimensional elastic stress analysis of a fracture specimen with an edge crack. Int. J. Fracture Mechanics, v.7, p.1-15.

DANSON, D.J. (1979). Analysis of plate bending problems by direct boundary element method. Southampton. M.Sc. (Dissertation) - Univerty of Southampton.

DOMINGUEZ, J. ; ALARCON, E. (1981). Elastodynamics. In: BREBBIA,C.A., ed. Progress in boundary element methods .London, Pentech Press, v.1

FERRO, N.C.P. (1993). Uma combinação MEC/MEF para análise de fundação enrijecidas por estacas. São Carlos. Dissertação (Mestrado) - Escola de Engenharia de São Carlos, Universidade de São Paulo.

FREDHOLM, I. (1906). Solution d'un Probème Fondamental de la Theorie de l'élasticité, Arkiv för Matematik, Astronomi och Fysk, v.2, n.28, p.3-8. 
FREDHOLM, J, (1903). Sur une classe d'equations fonctionelles. Acta Math., v.27, p.365-390.

GIL RODRIGUES, J.C.; VENTURINI, W.S. (1985). Boundary element technique for the analysis of tension discontinuity problems. In: BREBBIA, C.A.; NOYE, B.J., eds. BETECH 85, Berlin, Springer-Verlag.

GIL RODRIGUES, J.C.; VENTURINI, W.S. (1986). Sobre a influência da discretização de domínio bidimensionais para o método dos elementos de contorno. In: CONGRESSO LATINO-AMERICANO SOBRE MÉTODO COMPUTACIONAIS PARA ENGENHARIA, 7., São Carlos, EESC-USP, Anais.

GUO-SHU, S. ; MUKERJEE, S. (1986). Boundary element method analysis of bending plates of arbitrary shape with general boundary conditions. Eng. Analysis, v. 3, n. 1, p. $36-44$.

GUPTA, K.K.; MEEK, J.L. (1996). A brief history of the beginning of the finite element method. International Journal for Numerical Methods in Engineering. v.39, p.3761-3774.

HARTMANN, F. (1989). Introduction to boundary elemenis: theory and application. Berlin, Springer-Verlag.

HARTMANN, F. ; ZOTEMANTEL, R. (1986). The direct boundary element method in plate bending. Int. Num. Meth. Eng., v.23, n.11, p.2049-2069.

HATMANN, F. (1988). Kirchhoff plates. In: BREBBIA,C.A. ed.. Boundary element X. C.M. Publ.. v.3, p.409-423.

JASWON, M.A. (1963). Integral equation methods in potencial theory, I. Proc. Royay Society, A,(275): 237-246.

JASWON, M.A. ; MAITI, M. (1968). An integral formulation of plate bending problems. J. Engng. Math., v.2, p.83-93.

JASWON, M.A. ; MAITI, M. ; SYMM, G.T. (1967). Numerical biharmonic analysis and some applications. Int. Journal of Solids and Structures, v.3, p.309-312. 
KAMIYA, N. ; SAWAKI, Y. (1984). Finite deflection of plates. In: BREBBIA.C.A. ed.. Topics in boundary element research. Heil de berg, Springer-Verlag.

KAMIYA, N. ; SAWAKI, Y. ; NAKAMURA, J. (1982). Boundary element nonlinear bending analysis of clamped sandwich plates and shells. In: BREBBIA,C.A. ed.. Procedings of 4th Int. Seminar; Southampton, p.515-525.

KARAM, V.J. (1986). Aplicação do método dos elementos de contorno à teoria de Reissner para flexão de placas. Rio de Janeiro. Dissertação (Mestrado) Universidade Federal do Rio de Janeiro, COPPE.

KARAM, V.J. ; TELLES, J.C.F. (1990). Comparação entre as teorias de Kirchhoff e Reisner para análise de flexão de placas pelo MEC. In: XI CONGRESSO IBERO LATTINO ÁMERICANO SOBRE MÉTODO COMPUTACIONAIS PARA ENGENHARIA - COPPE - UFRJ, 1990, Anais.

KATSIKADELIS, J.T. (1991). Large deflection analysis of plates on elastic foundation by the boundary element method. Int. J. Solids and Structures, v.27, n. 15, p.1867-1878.

KATSIKADELIS, J.T. ; KALLIVOKAS, L.F. (1988). Plates on biparametric plastic foundation by BIDE method. Journal of Engineering Mechanics, v.114, n.5, p. $847-875$.

KATSKADELIS, J.T. ; ARMENAKKAS, A.E. (1984a). Plates on elastic foundaions by BIE method. Journal of Engineering Mechanics, ASCE, v.110, n.7, p.10861104.

KATSIKADELIS, J.T. ; ARMENÀKAS, A.E. (1984b). Analysis of clamped plates on elastic foundation by the boundary integral method. Journal of applied Mechanics, v. 54, p.544-580.

KATSIKADELIS, J.T. ; ARMENAKAS, A.E. (1989). A new boundary equation solution to the problem. J. Applied Mechanics, v.56, n.2, p.364-374.

KATSIKADELIS, J.T. ; KALLIVOKAS, L.F. (1986). Clamped plates on pasternak type elastic foundation by BEM. J. Applied Mechanics. v.53, n.4, p.909-917. 
KATSIKADELIS, J.T. ; NERANTZAKI, M.S. (1988). Large deflection of thin plates by BEM. In: BREBBIA,C.A. ed.. Boundary element $X$.

KIRCHHOFF, G. (1850). Über das gleichgewicht und die bewegung einer elastischen scheibe. Crelles J., v.40, p.51-88.

KUPRADZE, V.D. (1965). Potencial Methods in the Theory of Elasticity. Jerusalem, Israel Program for Scientific Translations.

LACHAT, J.C. (1975). A further development of the boundary integral technique for elastostatics. Southampton. (Ph..D. Thesis), University of Southampton.

LIOUVILLE, V.D. (1937). Sur le développment des fonctions. Journal de Mathématiques Pures et Appliqées, v.2, p.16-35.

LOVE, A.E.H. (1944). A treatise on the mathematical theory of elasticity. London, Cambridge University Press.

MANOLIS, G.D. ; BESKOS, D.E. (1981). Dynamic stress concentration studies by boundary integrals on Laplace Transform. Int. J. Num. Meth. Eng., v.17, p.573599.

MANZOLI, O.L. (1992). Formulação do método dos elementos de contorno para placas sobre fundação elástica. São Carlos. 142p. Dissertação (Mestrado) Escola de Engenharia de São Carlos, Universidade de São Paulo.

MENDELSON, A. (1968). Plasticity: Theory and application. New York, Robert E. Krieger Publishing Company.

MIKHLIN, S.G. (1957). Integral equations. New York, Pergamon Press. (Intenational series of monographs in pure and applied)

MIKHLIN, S.G. (1965). Multidimensional singulen integrals and integral equations. London, Pergamon Press.

MINDLIN, R.D. (1951). Influence of rotary inertia and shear on flexural motions of isotropic elastic plates. J. Appl. Mech., v.18, p.31-38. 
MORJARIA, M. ; MUKERJEE, S. (1980). Inelastic analysis of transverse deflection of plates by boundary element method. Journal of Applied Mechanics, v.47, n.2, p.291-296.

MORJARIA, M. ; MUKERJEE, S. (1981). Numerical analysis of planar time dependent inelastic deformation of plates with crack by the boundary element method. Int. J. Solids and Structures, v.17, p.127- .

MOSHAIOV, A. ; VORUS, W.S. (1986). Elastic-plastic bending analysis by a boundary element method with initial plastic moments. Int. J. Solids and Structures, v.22, n.11, p.1213-1229.

MUKERJEE, S. ; MORJARIA, M. (1981). A boundary element analysis of time dependent inelastic deformation of cracked plates loaded in antiplane shear. Int. $J$. Solids and Strustures, v.17, p.253-.

MUKERJEE, S. ; MORJARIA, M. (1981). A boundary element formulation for planar time dependent inelastic deformation of plates with cutouts. Int. J. Solids and Structures, v.17, p.115-126.

MUKHERJEE, S. ; CHANDRA, A. (1984). Boundary element formulation for large strain- large deformation problems of plasticity and viscopasticity. In: BANERJEE, P.K. ; MUKHERJEE, S., eds. Developments in boundary element methods, 3. Appl. Sci. Publ.

MUKHERJEE, S. ; RAJIYAH, H. (1987). Analysis of metal forming problems by the boundary element method. In: BREBBIA, C.A. ; VENTURINI,W.S., eds., Boundary element techniques: applications in stress analysis and heat iransfer. CML Publ.

MUSKHELISHVILI, N.I. (1953). Some basic problems of Mathematical theory of elasticity. Groningen Holand, Noordhoff.

NERANTZAKI, M.S. (1995). Analysis of plates with variable thickness by analog equation method. In: BREBBIA, C.A. et alii eds. Boundary elements XVII. Southampton, Computational Mechanics Publications. p.175-184. 
NOWAK, A.J.; BREBBIA, C.A. (1989). The multiple-reciprocity method. A new approach for transforming BEM domain integral to the boundary . Engeneering Analysis with Boundary Elements, v.6, n.3, p.164-167.

OLIVEIRA NETO, L. (1991). Análise de placas de borda curva pelo método dos elementos de contorno. São Carlos. 118p. Dissertação (Mestrado) - Escola de Engenharia de São Carlos, Universidade de São Paulo.

PAIVA, J.B. (1978). Formulação do método dos elementos de contorno para a flexão de placas e suas aplicação em engenharia de estruturas. São Carlos. 195p. Tese (Doutorado) - Escola de Engenharia de São Carlos, Universidade de São Paulo.

PILKEY, W.D. ; WUNDERLICH, W. (1992). Minechanics of Siructires variationat and computacional methods. USA, CRC Press.

PILTNER, R. ; TAYLOR, R.L. (1989). The solution of plate bending problems with the aid of a boundary element algorithms based a singular complex functions. In: TANAKA,M.;BREBBIA,C.A.;HONMA,T. ed.. Boundary elements XII.

PROVIDAKIS, C.P. ; BESKOS, D.E. (1989). Free and forced vibration of plates by boundary elements. Comput. Meth. Appl. Mech. Eng., v.74, n.3, p.231-250.

PROVIDAKIS, C.P. ; BESKOS, D.E. (1989). Free and forced vibration of plates by boundary and interior elements. Int. J. Num. Meth. Eng. , v.28, n.9, p.1977-1994.

REISSNER, E. (1944). On bending of elastic plates, J. Math. Physics, n.23, p.184191.

REISSNER, E. (1945). The effect of transverse shear deformation on the bending of elastic plates. J. Appl. Mech., v.12, p.69-77.

RIBEIRO, G.O. (1992). Sobre a formulação do método dos elementos de contorno para a flexão de placas usando as hipótese de Reissner. São Carlos. 265p. Tese (Doutorado)- Escola de Engenharia de São Carlos, Universidade de São Paulo. 
RIBEIRO, G.O. ; VENTURINI, W.S. (1989). Aplicação do método dos elementos de contorno no cálculo de placas através da teoria de Reissner. In: CONGRESSO IBERO-LATINO-AMERICANO SOBRE MÉTODO COMPUTACIONAIS EM ENGENHARIA, 10., Porto, Portugal. Anais.

RICCARDELA, P.C. (1973). An implementation of the boundary integral technique for planar problems in elasticity and elastopasticity. Pittsburg, Dept. Mech. Engng., Carnegie Mellon University, (Report No. SM-73-10)

RIZZO, F.J. (1967). An Integral equation approach to boundary value problems of classical elastostatics. Quarterly of Applied Mathematic, v.25, n.1, p.83-95.

RIZZO, F.J. ; SHIPPY, D.J. (1968). A formulation and solution procedure for the generai non-homogeneous elastic inclusion problem. Inî. J. Solids and Strucures, v.4, p. 1161-1179.

ROBINSON, J. (1985). Early FEM pioners. Robinson and Associates. England. Dorset.

SAPOUNTZAKIS, E.J. ; KATSIKADELIS, J.T. (1991). Boundary element solution of plates of variable thickness. J.Eng. Mech., v.117, n.6, p.1241-1256.

SAWAKI, Y. ; TAKEUCHI, K. ; KAMIYA, N. (1989). Finite deflection analysis of plates by dual reciprocity boundary elements. In: BREBBIA,C.A.; CONNOR,J. ed.. Advances in boundary elements: vol. 3- Stress analysis. Berlin, SpringerVerlag.

SECHLER, E.E (1952). Elasticity in engineering. New York, John Wiley \& Sons. SILVA, N.A. (1988). Aplicaçâo do método dos elemeníos de contorno à análise de placas sobre fundação elástica. São Carlos. 162p. Dissertação (Mestrado) Escola de Engenharia de São Carlos, Universidade de São Paulo.

SILVA, N.A. (1996). Aplicação do método dos elementos de contorno às piacas com enrijecedores utilizando a teoria de Reissner. São Carlos. 219p. Tese (Doutorado) - Escola de Engenharia de São Carlos, Universidade de São Paulo.

SMIRNOV, V.J. (1964). Integral equations and partial differential equations. In: COURSE in higher mathematics. London, Addison -Wesley, v.4. 
SOKOLNIKOFF, I.S. (1956). Mathematic theory of elasticity. New York, McGraw-Hill.

SOUTHWELL, R.V. (1946). Relaxation methods in theoretical physics. London, Oxford. University Press, (The Oxford Engineering Sciencs Series).

STERN, M. (1979). A general boundary integral formulation for the numerical solution of plate bending problems. Int. Journal of Solids and Structures, v.15, p.769-782.

STERN, M. (1983). Boundary integral equations for bending of thin plates. In: BREBBIA, C.A. ed.. Progress in boundary element, v.2 .London, Pentech Press.

SWEDLON, J.L. ; CRUSE, T.A. (1971). Formulation of boundary integral equations for three dimensional elastoplastic flow. Int. J. Solids and Sirictures, v. 7, p.16731683

SYMM, G.T. (1963). Integral equation methods in potencial theory, II. Proc. Royal Society $A,(275): 33-46$.

TANAKA, M. (1982). Integral equation approach to small and large displacements of thin elastic plates. In: BREBBIA, C.A. ed.. Boundary elements in engineering. Proc. 4th. Int. Seminar, Heil de berg, Springer-Verlag.

TEJERINA CALDERÓN, E. (1991). Uma formulação aliernativa parta o estudo de placus sobre fundafão elástica pelo método dos elementos de contorno. São Carlos. 191p. Dissertação (Mestrado) - Escola de Engenharia de São Carlos, Universidade de São Paulo.

TEJERINA CALDERÓN, E. (1996). Sobre o uso do método dos elementos de contorno-MEC para o estudo de interação de placas com o meio contínuo. São Carlos. 247p. Tese (Doutorado) - Escola de Engenharia de São Carlos, Universidade de São Paulo.

TIMOSHENKO, S.P. ; GOODIER, J.N. (1980). Teoria da elasticidade. Rio de Janeiro, Guanabara Dois S.A. 
TOTTENHAM, H. (1979). The boundary element method for plates and shells, In:BANERJEE,P.K. ; BUTTERFIELD, R. eds. Developments in boundary element methods. London, Elsevier, p.173-205.

TURNER, M.J.; CLOUGH, R.W.; MARTIN, H.C.; TOPP, L.J. (1956). Stifnerss and deflection analysis of complex structures. Journal Aeronaut Sciences, v.23, n.9, p. 805-823

UGURAL, A.C. ; FENSTER, S.K. (1981). Advanced strength and applied elasticity, The SI version. London, Elsevier Science Publishimg Co.,Inc

VENTURINI, W. S. (1982). Application of the boundary element formulation to solve geomechanical problems. Southampton. (Ph.D.Thesis). University of Southampton.

VENTURINI, W.S. ; BREBBIA, C.A. (1984). Boundary element formulation for nonlinear applications in geomechanics. Appl. Math. Modelling, v.8, p.251-260.

VITOORAPORN, C. ; MOSHAIOU, A. (1989). Effectiveness study of higer order elements in the BIE method for thin elastic plate bending. In: TANAKA, M. ; BREBBIA, C.A. ; HONMA, T. ed.. Boundary elements XII.

WEATPHAL JR. , T. ; BARCELLOS, C.S. (1989). Applications of the boundary element method to Reissner's and Mindlin's plate method modeis. In: TAINAKA,M.; BREBBIA, C. A. ; HONMA, T. eds. Boundary elements XII.

WEEËN, Van Der F. (1982a). Application of the direct boundary element method to Reissner's plate model. In: BREBBIA,C.A. ed.. Boundary element methods in engineering: Procedings of the Fourth International Seminar, Southampton, England, Sept.

WEEËN, Van Der F.(1982b). Application of boundary integral equation method to Reissner's plate model. Int. J. Num. Meth. Eng., v.18, n.1, p.1-10.

WOLF, J.P. (1985). Dynamic soil-structure interaction. Prentice Hall.

WU, B.C. ; ALTIERO, N.J. (1979). A boundary integral method applied to plates of arbitrary plan form and arbitrary boundary conditions. Computers \& Structures, v.10, p.107-707. 
XIAO-YAN, L. ; KUANG-MAO, H. ; XIUXI, W. (1990). Geometrically non-linear analysis of a Reissner type plate by the boundary element method. Computers \& Structures, v.37, n.6, p.911-916.

YE, T.Q. ; LIN, Y. (1985). Finite deflection analysis elastic plate by the boundary element method. Appl. Math-Modelling, v.9, p.183-188.

ZIENKIEWICZ, O.C. ; KELLY, D.W. ; BETTESS, P. (1977). The coupling of the finite element method and boundary solution procedures. Int. Journal for Numerical Methods in Engineering. v.11, p.355-375. 